\title{
Time-varying spectral analysis on Hilbert spaces
}

\author{
Citation for published version (APA):
}

van Delft, A. M. N. (2016). Time-varying spectral analysis on Hilbert spaces: theory and practice. [Doctoral Thesis, Maastricht University]. Universitaire Pers Maastricht. https://doi.org/10.26481/dis.20161219ad

Document status and date:

Published: 01/01/2016

DOI:

10.26481/dis.20161219ad

Document Version:

Publisher's PDF, also known as Version of record

Document license:

Unspecified

\section{Please check the document version of this publication:}

- A submitted manuscript is the version of the article upon submission and before peer-review. There can be important differences between the submitted version and the official published version of record.

People interested in the research are advised to contact the author for the final version of the publication, or visit the DOI to the publisher's website.

- The final author version and the galley proof are versions of the publication after peer review.

- The final published version features the final layout of the paper including the volume, issue and page numbers.

Link to publication

\footnotetext{
General rights rights.

- You may freely distribute the URL identifying the publication in the public portal. please follow below link for the End User Agreement:

www.umlib.nl/taverne-license

Take down policy

If you believe that this document breaches copyright please contact us at:

repository@maastrichtuniversity.nl

providing details and we will investigate your claim.
}

Copyright and moral rights for the publications made accessible in the public portal are retained by the authors and/or other copyright owners and it is a condition of accessing publications that users recognise and abide by the legal requirements associated with these

- Users may download and print one copy of any publication from the public portal for the purpose of private study or research.

- You may not further distribute the material or use it for any profit-making activity or commercial gain

If the publication is distributed under the terms of Article $25 \mathrm{fa}$ of the Dutch Copyright Act, indicated by the "Taverne" license above, 


\section{Time-varying spectral analysis on Hilbert spaces:}

\section{Theory and practice}

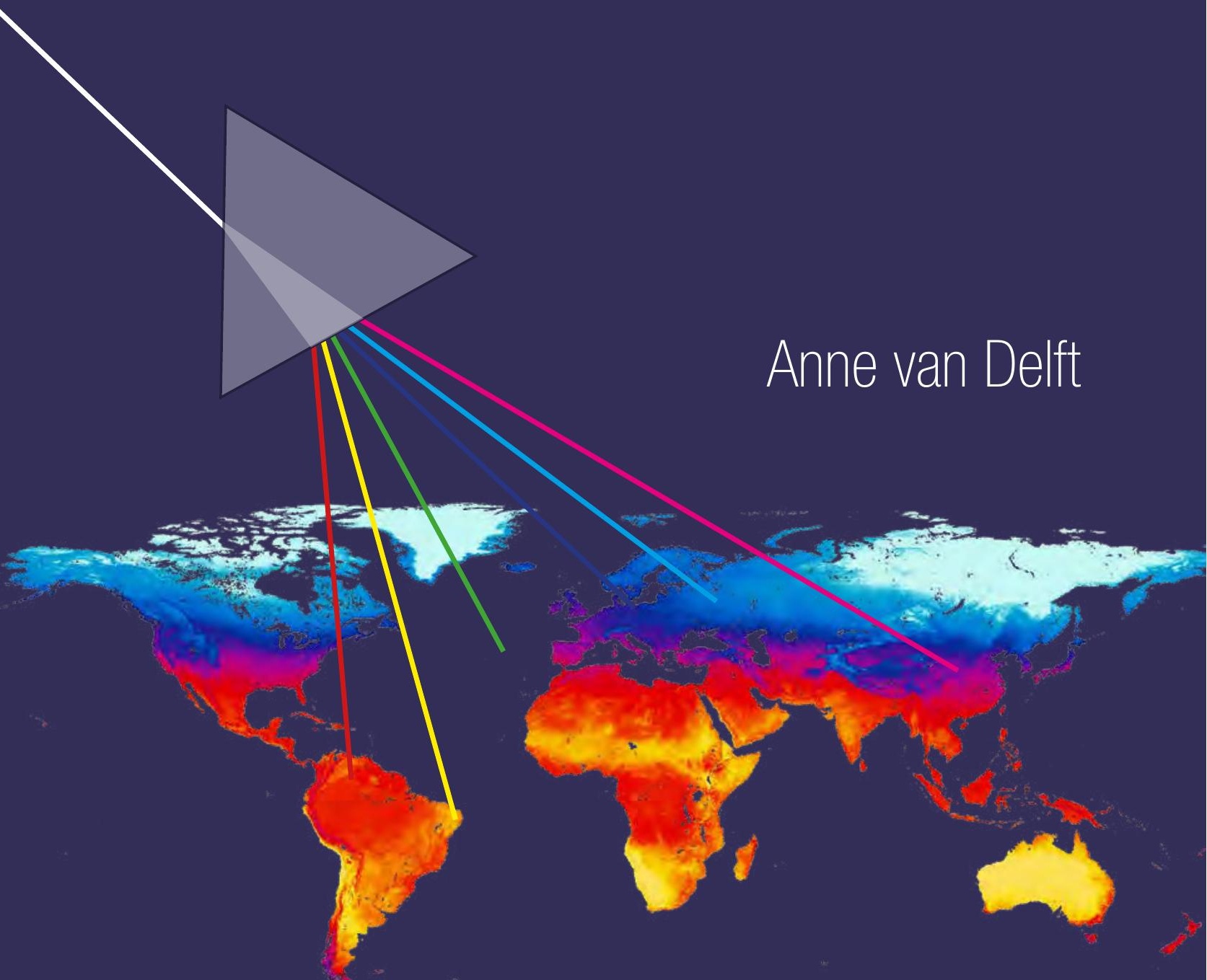





\title{
TIME-VARYING SPECTRAL ANALYSIS ON HILBERT SPACES: THEORY AND PRACTICE
}

\author{
DISSERTATION
}

to obtain the degree of Doctor at Maastricht University, on the authority of the Rector Magnificus,

Prof. dr. Rianne M. Letscher, in accordance with the decision of the Board of Deans, to be defended in public on Monday December $19^{\text {th }}, 2016$ at 15.45 hours

by

Anne Margrete Nicolien van Delft 


\section{Promotores:}

Prof. dr. J.R.Y.J. Urbain $\dagger$

Prof. dr. H.J.M. Peters

\section{Co-promotor:}

Dr. M. Eichler

Beoordelingscommissie:

Prof. dr. F.C. Palm (voorzitter)

Dr. E.A. Beutner

Dr. S. Hörmann (Université libre de Bruxelles)

Prof. dr. R. von Sachs (Université catholique de Louvain) 
Copyright (C) Anne M.N. van Delft, 2016

All rights reserved. No part of this publication may be reproduced, stored in a retrieval system, or transmitted in any form, or by any means, electronic, mechanical, photocopying, recording or otherwise, without the prior permission in writing from the author.

This book was typeset by the author using $\mathrm{L}_{\mathrm{A}} \mathrm{T}_{\mathrm{E}} \mathrm{X}$.

Published by Universitaire Pers Maastricht

ISBN: 978-94-6159-642-0

Printed in The Netherlands by Datawyse Maastricht 
In dear memory of my promotor Jean-Pierre Urbain 


\section{Acknowledgements}

When I first came to Maastricht, I thought it would only be for a short while. My estimation was slightly off and I only left Maastricht a few days ago, more than eight years later ${ }^{1}$. Two people played a major role in this: my promotor JeanPierre Urbain and co-promotor Michael Eichler, who both made me enthusiastic for research. I could therefore not imagine a more bitter way to leave Maastricht and finishing the last few words of my dissertation than with the tragic and sudden passing of Jean-Pierre, at the beginning of October, still fresh on my mind.

There are several people that I would like to express my gratitude to. Firstly, I would like to thank Michael for being my advisor and for introducing me to new and interesting topics in statistics and mathematics. I moreover like to thank him for his patience necessary to deal with my slightly chaotic nature and for sharing his vast amount of knowledge with me. I plan to continue our lame math jokes during conferences and I hope we will continue to work together in the future.

Secondly, I am very grateful to Franz Palm, Eric Beutner, Siegfried Hörmann and Rainer von Sachs for having accepted to be part of my assessment committee and for their useful comments and feedback. In this respect, I would especially like to express my gratitude to Franz Palm, the Chair of the committee. Additionally, I would like to thank the Dean Philip Vergauwen and Head of our department Hans Peters for finding a suitable solution to the unusual situation that arose in October.

I would like to thank Alexander Aue for the invitation to come to UC Davis for a research visit. It has been a true pleasure working with him. I really appreciate the great advices given and the laughs we shared. I am looking forward to continue our collaboration and to further develop the ideas that ensued from the first project. I am moreover very grateful to Rainer von Sachs, Rainer Dahlhaus, Holger Dette, François Roueff and Victor Panaretos for the opportunity to share my research and for the nice discussions that I have had with them during conferences.

On a personal level, special thanks to my (ex-) colleagues Brigitte, Yolanda, Karin, Anna, Carlos, Kouma, Ahmad, Thomas, Dennis and Lenard, to my mother and to my lovely (slightly crazy) friends for the fun times and for always being there for me.

Finally, I would like to end with a few words about Jean-Pierre, whom I feel deeply indebted to. I first encountered him as my teacher for the course 'Time Series', where I occasionally drifted off during the early morning lectures. Although he generally treated me on an amused grin when this occurred, I think this is why

\footnotetext{
${ }^{1}$ My study abroad in Australia did provide the necessary change of scenery.
} 
he often called me to come to the board to solve problems classically during the tutorials. These hours in front of the board did however not feel like punishment but made me passionate about time series analysis and in particular asymptotic theory. During my years as a $\mathrm{PhD}$ candidate, I had many random and interesting discussions with Jean-Pierre. I am going to miss the philosophical ones, which oftentimes concerned education and the role of universities in society. I will also miss the unasked general life lessons he sent me home with to ponder on. But mostly I am going to miss our conversations about music and the great passion we shared for it. Thank you for your trust, for believing in me and for being someone I could trust.

Anne van Delft

Louvain-la-Neuve, November 2016 


\section{Contents}

Acknowledgements iv

1 Introduction 1

1.1 Hilbert space for functional data . . . . . . . . . . . . . . . . . . . . . . . 3

1.2 Spectral domain analysis . . . . . . . . . . . . . . . . . . 5

1.2.1 Weakly stationary time series . . . . . . . . . . 6

1.2.2 Time-varying spectral analysis . . . . . . . . . . . . . . . . . . . . . . . 11

1.2.3 The contribution of this thesis . . . . . . . . . . 15

2 Data-adaptive estimation of time-varying spectral densities $\quad 19$

2.1 Introduction . . . . . . . . . . . . . . . . . . . . . . 19

2.2 Theoretical framework: Locally stationary processes . . . . . . . . . . 21

2.3 Propagation-separation approach in the time-frequency plane . . . . 23

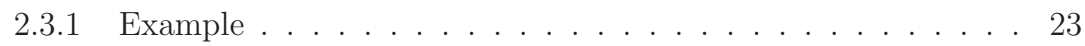

2.3.2 A local estimator: the modified pre-periodogram . . . . . . . 24

2.3.3 Adaptive estimation of the time-dependent spectrum . . . . . 26

2.3.4 Penalty step . . . . . . . . . . . . . . . . . . . . . . . . . . . . . . . . . . 38

2.3.5 The memory step . . . . . . . . . . . . . . . . . . . . . . . . . . . . . . . 31

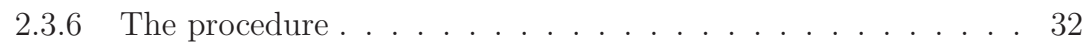

2.3.7 Computational complexity . . . . . . . . . . . . . . . 33

2.3.8 Asymptotic considerations . . . . . . . . . . . . . . . 34

2.4 Specification of parameters . . . . . . . . . . . . . . . . . . . . . . . . . . . . . 37

2.4.1 Bandwidth parameters . . . . . . . . . . . . . . 37

2.4 Kernels . . . . . . . . . . . . . . . . . . . 40

2.5 Examples . . . . . . . . . . . . . . . . . . . . . 40

2.5.1 Structural break white noise . . . . . . . . . . . . . 41

2.5.2 Locally stationary series . . . . . . . . . . . . . . . . . . . . . . . . . . . . 42

2.5.3 Structural break in locally stationary series . . . . . . . . . . . . 42

2.6 Application to Local Field Potentials . . . . . . . . . . . . . . . . . . 48

2.7 Conclusion . . . . . . . . . . . . . . . . . . 50 50

3 Locally stationary functional time series 53

3.1 Introduction . . . . . . . . . . . . . . . . . . . 53

3.2 Locally stationary functional time series . . . . . . . . . . . . . . . . . 55

3.2.1 Functional spaces and operators . . . . . . . . . . . 56 
3.2.2 Cramér representation for strictly stationary processes . . . . 57

3.2.3 Existence of stochastic integral . . . . . . . . . . . . . . . 59

3.2.4 Local stationarity in the frequency domain . . . . . . . . . . . 61

3.3 Time-varying spectral density operator . . . . . . . . . . . . 63

3.4 Locally stationary functional autoregressive processes . . . . . . . . . 68

3.5 Estimation . . . . . . . . . . . . . . . . . . . 75

3.5.1 The functional segmented periodogram . . . . . . . . . . 75

3.5.2 Consistent estimation . . . . . . . . . . . . . . . . . 78

3.5.3 Weak convergence of the empirical process . . . . . . . . . . 82

3.6 Numerical simulations . . . . . . . . . . . . . . . . . . . . . . . 84

3.7 Conclusion . . . . . . . . . . . . . . . . . . . . . . . . . . . . 90

A.1 Some operator theory . . . . . . . . . . . . . . . . . . . . . . . 93

A.2 Higher order dependence . . . . . . . . . . . . . . . . . . . . . 97

A.2.1 Moment tensors on separable Hilbert spaces . . . . . . . . . . 97

A.2.2 Linear transformations of elementary tensors . . . . . . . . . . 98

A.3 Data taper . . . . . . . . . . . . . . . . . . . . . . . . . 101

A.4 Proofs . . . . . . . . . . . . . . . . . . . 103

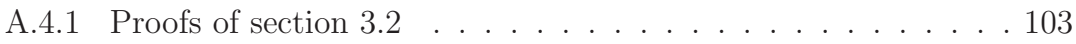

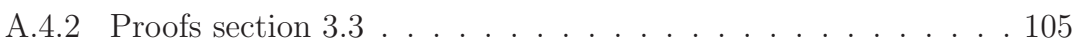

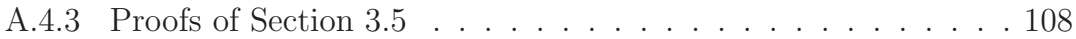

4 Testing for stationarity of functional time series in the frequency $\begin{array}{lr}\text { domain } & 117\end{array}$

4.1 Introduction . . . . . . . . . . . . . . . . . . . . . 117

4.2 Notation and setup . . . . . . . . . . . . . . . . . . . 118

4.2.1 The function space . . . . . . . . . . . . . . . . . . . . . 118

4.2.2 Dependence structure on the function space . . . . . . . . . 120

4.2.3 The functional Discrete Fourier Transform . . . . . . . . . . . 121

4.3 The functional stationarity testing framework . . . . . . . . . . . 122

4.4 Large sample results . . . . . . . . . . . . . . . . . . . . . . . . . 124

4.4.1 Properties under the null of stationarity . . . . . . . . . . . 124

4.4.2 Properties under the alternative . . . . . . . . . . . . 126

4.5 Empirical results . . . . . . . . . . . . . . . . . . . . . 130

4.5.1 Simulation setting . . . . . . . . . . . . . . . . . . . . . . . . . . . . . . . . . . . .

4.5.2 Finite sample performance under the null . . . . . . . . . . 131

4.5.3 Finite sample performance under the alternative . . . . . . . . 133

4.5.4 Toward an automated test procedure . . . . . . . . . . 135

4.6 Conclusion . . . . . . . . . . . . . . . . . . . . . . . . . 141

B.1 Properties of functional cumulants . . . . . . . . . . . . . . . . 143

B.2 Error bound for the denominator of the test statistic . . . . . . . . 148

B.3 Convergence of finite dimensional distributions . . . . . . . . . . . 155

B.4 Weak convergence. . . . . . . . . . . . . . . . 157

B.5 Proofs under the null hypothesis of stationarity . . . . . . . . . . . 159

B.6 Proofs under the alternative hypothesis of local stationarity . . . . . 163 
5 Conclusion 171

Bibliography 177

Valorization 183

Nederlandse samenvatting 187

Curriculum Vitae 191 



\section{Chapter 1}

\section{Introduction}

There is a doctrine in Buddhism called 'Anicca'. A rough translation of this doctrine asserts that "all temporal things are in a constant state of change". Although probably not intended as such originally, this certainly applies to most random processes that arise in statistical applications. The assumption that the probabilistic structure of a stochastic process stays constant over time is often not justified in practice. Many physical phenomena exhibit nonstationary behavior as a result of smooth changes in their second-order dependence structure over time. Examples can found in a wide range of disciplines such as geophysics, medicine, economics and engineering $^{1}$. In addition to this, a wide range of modern datasets that show this type of behavior can be viewed as being recordings from processes that vary over a continuum. For example, in meteorology daily records of temperature, precipitation and cloud cover over a region as three related surfaces may change over time due to global climate changes. The main object of this thesis is the study of stochastic processes that are characterized by both aspects.

The violation of the assumption of weak stationarity - the invariance to translations in time up to second-order - has two important implications. Firstly, a spectral theory is not necessarily well-defined. Time-invariance of the second-order structure of a weakly stationary process ensures that the process can equivalently be represented in the spectral domain, where the random process is given as a function of frequency rather than as a function of time. The spectral domain representation (Cramér, 1942) can be viewed as a stochastic analogue to the Fourier representation. It provides information of the contribution of the various frequency components to the total variation or 'energy' of the process via the spectral distribution function or, if it exists, via its corresponding spectral density function. For nonstationary processes, a spectral theory in which the physical interpretation of concepts such as 'energy' and 'frequency' are preserved, is generally not possible. It can however be established for processes of which the nonstationary characteristics change slowly over time (Priestley, 1965). The spectral distributions then become local in nature and can be captured via a time-dependent spectrum. The time-dependent spectrum and the time-dependent spectral density operator (Chapter 3), the generalization to the infinite dimension, play a central role in the remainder of this thesis.

\footnotetext{
${ }^{1}$ See also the Valorization Addendum provided at the end of this dissertation
} 
Secondly, an alternative asymptotic theory is necessary for meaningful statistical inference. Properties of many statistical inference procedures are derived using an asymptotic theory for which the assumption of weak stationarity lies at the basis. This classical asymptotic theory asserts that an increasing sample size will provide more information on the structure of the process. However, if the structure changes over time, future observations might not be of the same kind thereby invalidating results derived in such framework. An alternative asymptotics can be achieved by considering double-indexed processes that have time-dependent characteristics in combination with an infill asymptotic approach that rescales time. An increasing sample size then yields more information of the local structure of the underlying nonstationary process. This was first introduced for the class of locally stationary time series (Dahlhaus, 1996a). Time series that belong to this class exhibit smooth changes in dependence structure such that they are approximately stationary on a sufficiently small time interval. The latter ensures the existence of a well-defined representation in the spectral domain, where the frequency distribution of the signal is changing slowly but continuously in time. The changing second-order dependence structure of the process can therefore be completely characterized via its timedependent spectrum.

This thesis is concerned with the development of theory and methodology for analyzing stochastic processes $\left\{X_{t}: t \in \mathbb{Z}\right\}$ of which the spectral characteristics are changing over time. The elements of the stochastic process take values in a separable Hilbert space and are therefore random functions. Stochastic processes of this type are known in the literature as functional time series, a research topic that is still in its infancy. Due to the technological advances many datasets can nowadays be considered as being sampled recordings from processes of which the elements vary over a continuum such as a curve or a surface. Techniques to model these appropriately has therefore gained importance and statistical methods and tools for weakly stationary functional time series are now reasonably well-developed. As already mentioned above, the assumption of weak stationarity is a questionable one in many applications. Part of the contribution of this thesis is to relax this by introducing the concept of local stationarity for functional time series (Chapter 3).

The structure of this thesis is as follows. The remainder of this chapter provides some more background on the Hilbert space and on spectral domain analysis. This is followed by an overview of the three subsequent chapters. The three subsequent chapters form the main contribution of this thesis. In Chapter 2, the problem of estimation of time-dependent spectra is addressed and the design and implementation of an algorithm for data-adaptive kernel estimation of time-varying spectra with structural breaks is provided. In Chapter 3, spectral theory and methodology is developed to model functional time series that have time-varying spectral characteristics. In Chapter 4, a spectral domain-based method is proposed to test for weak stationarity of a functional time series, where the distribution of the test statistic is moreover established under smooth alternatives. 


\subsection{Hilbert space for functional data}

\section{The function space $H$}

This thesis is mainly concerned with stochastic processes of which the elements are themselves functions. The analysis of random functions, not necessarily an ordered collection, is also more generally known as functional data analysis. Functional data is often assumed to take values in a separable Hilbert space. The function space that is considered in this thesis is the Lebesgue space of equivalence classes of square integrable functions on $[a, b]$. That is, $H=L^{2}([a, b], \mathscr{B}, \lambda)$ is the complex vector space endowed with the inner product

$$
\langle x, y\rangle=\int_{a}^{b} x(\tau) \overline{y(\tau)} d \tau, \quad x, y \in H .
$$

The induced norm is denoted by $\|\cdot\|_{2}$. The closed interval $[a, b]$ is chosen for simplicity but could be any compact subset of a complex vector space. The choice for this particular function space is easily motivated. The general space of functionvalued random variables is itself too big and complex to be able to consider statistical procedures in a meaningful way. The analysis of functional data therefore requires imposing necessary structure on the space where the random functions can take their values. The choice of a Hilbert space $H$ - i.e., an inner product space that is complete - is then a natural one as it provides structure via its inner product and allows to consider geometrical notions such as length and angle. In addition, the intrinsic variation of a function-valued random variable is uncountably infinite. This can be restricted to countably finite in case the function space is separable and hence admits a countable orthonormal basis. Because all separable Hilbert spaces are isometrically isomorphic to the sequence space $\ell^{2}$, the particular choice is not too important. However, the space of square integrable functions can be considered the least restrictive one and has become widely accepted in the literature on functional data analysis.

\section{Linear mappings on separable Hilbert spaces}

An operator is a linear mapping from a function space into another function space. A linear operator $A: H_{1} \rightarrow H_{2}$ is said to be bounded if there exists a constant $K$ such that

$$
\|A x\|_{H_{2}} \leqslant K\|x\|_{H_{1}} \quad \forall x \in H_{1} .
$$

A linear operator is bounded if and only if it is continuous. A bounded linear operator between two Hilbert spaces $H_{1}, H_{2}$ is called compact if it can be represented as

$$
A=\sum_{n=1}^{\infty} s_{n}(A) \psi_{n} \otimes \phi_{n},
$$

where $\left\{s_{n}(A)\right\}_{n \geqslant 1}$ is a decreasing sequence of positive numbers that converges to zero and $\psi_{n}$ and $\phi_{n}$ are orthonormal bases of $H_{1}$ and $H_{2}$, respectively. The bounded linear operator $\psi_{n} \otimes \phi_{n}$ is defined as $\left(\psi_{n} \otimes \phi_{n}\right) x=\left\langle x, \phi_{n}\right\rangle \psi_{n}$ (Appendix A.1). Compact 
operators can be classified to belong to a particular Schatten class based on the properties of the singular values. That is, we say an operator belongs to the $p$-th Schatten class if its Schatten $p$-norm is finite, where the Schatten $p$-norm is defined as

$$
\|A\|_{p}=\left(\sum_{n \geqslant 1} s_{n}(A)^{p}\right)^{1 / p}<\infty \quad 1 \leqslant p<\infty .
$$

In other words, an operator is bounded in Schatten $p$-norm if and only if the singular values belong to a sequence space of order $\ell^{p}$. The Schatten class of order $p$, denoted by $S_{p}\left(H_{1}, H_{2}\right)$ together with the norm $\|\cdot\|_{p}$ forms a complete normed vector space i.e., a Banach space. Although bounded linear operators are not necessarily compact, it is convention to denote the space of these by $S_{\infty}\left(H_{1}, H_{2}\right)$ with standard operator norm $\|A\|_{\infty}=\sup _{n} s_{n}(A)$. A simple illustration of a bounded linear operator that is not compact in the infinite-dimensional case is the identity operator $I$ which maps any element $x \in H$ to the same element $x \in H$, i.e., $I x=x, x \in H$. If $H$ is infinitedimensional then the identity cannot be compact because all eigenvalues are equal to unity. It is however clearly bounded since $\|I\|_{\infty}=1$. In the special case $p=2$, $S_{2}\left(H_{1}, H_{2}\right)$ is a separable Hilbert space when equipped with the inner product

$$
\left\langle A_{1}, A_{2}\right\rangle_{H_{1} \otimes H_{2}}=\sum_{n \geqslant 1}\left\langle A_{1} \psi_{n}, A_{2} \psi_{n}\right\rangle \quad A_{1}, A_{2} \in S_{2}\left(H_{1}, H_{2}\right),
$$

where $\left\{\psi_{n}\right\}_{n \geqslant 1}$ is an orthonormal basis of $H_{1}$ and where the inner product does not depend on the choice of $\left\{\psi_{n}\right\}_{n \geqslant 1}$. The class $S_{2}\left(H_{1}, H_{2}\right)$ is called the class of HilbertSchmidt operators and its elements are kernel operators because they can be written as (Appendix A.1)

$$
A x(\tau)=\int_{a}^{b} a(\tau, \sigma) x(\sigma) d \sigma \quad \tau \in[a, b],
$$

where the function $a \in L^{2}\left([a, b]^{2}, \mathscr{B}, \lambda\right)$ is called the kernel of $A$ and $\|A\|_{2}=\|a\|_{2}$. Because of this bijective correspondence, the properties of Hilbert-Schmidt operators can be obtained via the kernel function which provides a useful relation to the $L^{p}$ spaces. Note how this transformation can be viewed as an analog to the multivariate world where the linear transformation of a $d$-dimensional vector $\underline{x}$ with the $d \times d$-dimensional matrix $\boldsymbol{A}$ is given by

$$
[\boldsymbol{A} \underline{x}]_{i}=\sum_{j=1}^{d} \boldsymbol{A}_{i j} \underline{x}_{j} \quad i=1, \ldots, d .
$$

Based on the definition of the Schatten $p$-norms, we have the inclusion

$$
S_{1}\left(H_{1}, H_{2}\right) \subset S_{2}\left(H_{1}, H_{2}\right) \ldots \subset S_{\infty}\left(H_{1}, H_{2}\right) .
$$

$S_{1}\left(H_{1}, H_{2}\right)$ is the strongest class of the Schatten class operators which are the nuclear or Trace-class operators. To ease notation, the space $S_{p}(H, H)$ is simply denoted by $S_{p}(H)$. A particular useful result is that if $1 \leqslant p, q, r \leqslant \infty$ for $r^{-1}=p^{-1}+q^{-1}$ and $A \in s_{q}(H), B \in S_{p}(H)$ then $A B \in S_{r}(H)$ and

$$
\|A B\|_{r} \leqslant\|A\|_{q}\|B\|_{p} .
$$


This is known as Hölder's Inequality for Schatten class operators and is helpful in the analysis of functions that involve multiple operators belonging to different classes.

\section{Random processes on Hilbert spaces}

Consider a stochastic process $\left\{X_{t}: t \in \mathbb{Z}\right\}$ defined on a probability space $(\Omega, \mathscr{A}, \mathbb{P})$ taking values in a separable Hilbert space $H$. Furthermore, let $\mathbb{H}=L^{2}(\Omega, H, \mathbb{P})$ denote the Hilbert space of the collection of equivalence classes of $H$-valued random variables on $\Omega$ with finite second moment. If $H=L^{2}([a, b], \mathscr{B}, \lambda)$, we say $X_{t}$ is an element of $\mathbb{H}$ if

$$
\mathbb{E}\left\|X_{t}\right\|_{2}^{2}=\int_{\omega \in \Omega}\left\|X_{t}(\omega)\right\|_{2}^{2} d \mathbb{P}(\omega)<\infty \quad t \in \mathbb{Z}
$$

The first two moments of $X_{t}$ for all $t \in \mathbb{Z}$ are well-defined in an $L^{2}$ sense. That is, the mean function $m_{t}$ of $X_{t}$ exists and is defined as the unique element of $H$ such that

$$
\left\langle m_{t}, y\right\rangle=\mathbb{E}\left\langle X_{t}, y\right\rangle \quad \forall y \in H
$$

while the $h$-th lag covariance operator between $X_{t}$ and $X_{t+h}$ is given by

$$
\mathcal{C}_{t+h, t}=\mathbb{E}\left[\left(X_{t+h}-m_{t+h}\right) \otimes\left(X_{t}-m_{t}\right)\right] \quad,
$$

which belongs to $S_{2}(H)$. If $\left\{X_{t}: t \in \mathbb{Z}\right\}$ is a weakly stationary process then the mean function and lag $h$ covariance operator are independent of $t$ and are given by $m$ and $\mathcal{C}_{h}$, respectively. Because $\left\{X_{t}\right\}$ takes values in $H$, it is possible to write the random function as the parametrization

$$
\tau \mapsto X_{t}(\tau) \quad \tau \in[a, b] .
$$

This makes it easier to see the analogy to the multivariate world. However, the point-wise evaluation is not necessarily well-defined unless the curves are bounded in supremum norm. For example, the kernel function $c_{h}$ of $\mathcal{C}_{h}$, which is an element of $L^{2}([a, b], \mathscr{B}, \lambda)$, can be written point-wise as

$$
c_{h}(\tau, \sigma)=\operatorname{cov}\left(X_{h}(\tau), X_{0}(\sigma)\right) .
$$

It should however be kept in mind that this equality only holds in an $L^{2}$ sense, i.e, $\left\|c_{h}-\operatorname{cov}\left(X_{h}, X_{0}\right)\right\|_{2}=0$.

\subsection{Spectral domain analysis}

In 1664, Isaac Newton passed sunlight through a triangular glass prism which decomposed the light into a rainbow of colors. Recombining with a second prism would provide the original white sunlight again. It is now well-known that visible light forms a small section of the electromagnetic spectrum. The experiment of Newton concerned breaking up a time series into its individual frequency components and can therefore be viewed as the first serious application of spectral analysis. In this 
section, we sketch some background on spectral domain analysis, which is often also referred as frequency domain analysis. In the remainder of this thesis, these terms are used interchangeably. This section starts with the main concepts for spectral domain analysis of finite-dimensional weakly stationary time series and its generalization to functional time series. A short review on the analysis of finite-dimensional time series with time-varying spectral characteristics is then provided. The section is concluded by the contribution of this thesis, which in particular generalizes timevarying spectral analysis to the infinite dimension.

\subsubsection{Weakly stationary time series}

Two results that lie at the core of spectral domain analysis for weakly stationary processes are Bochner's Theorem and the Spectral Representation Theorem. Roughly speaking, Bochner's Theorem asserts that among the complex-valued bounded continuous functions on $\mathbb{R}^{d}$, the non-negative definite functions are those which are the Fourier transforms of non-negative and finite Borel measures. This theorem can be used to describe the autocovariance of a weakly stationary time series. Let $H=\mathbb{C}$ and consider a zero-mean weakly stationary time series with autocovariance function

$$
\gamma(h)=\mathbb{E}\left[X_{t+h} \bar{X}_{t}\right] \quad h \in \mathbb{Z},
$$

where $\bar{X}_{t}$ is the conjugate of $X_{t}$. Because $\gamma(\cdot)$ is non-negative definite, Bochner's Theorem implies that there exists a right continuous, nondecreasing, bounded function $F \in \mathbb{C}$ on $[-\pi, \pi]$ with $F(-\pi)=0$ such that

$$
\gamma(h)=\int_{-\pi}^{\pi} e^{\mathrm{i} \omega h} d F(\omega)
$$

where i denotes the imaginary number. The function $F$ and its corresponding Radon-Nikodym derivative $f$ (if it exists) are referred to as the spectral measure and spectral density, respectively, of both the process $\left\{X_{t}\right\}$ and its autocovariance function $\gamma(\cdot)$. If the autocovariance function is absolutely summable, i.e., $\sum_{h \in \mathbb{Z}}|\gamma(h)|<\infty$, then the spectral distribution function $F$ is absolutely continuous and the autocovariance function can be written as

$$
\gamma(h)=\int_{-\pi}^{\pi} e^{\mathrm{i} \omega h} f(\omega) d \omega .
$$

The autocovariance function of $\left\{X_{t}\right\}$ at lag $h$ can be viewed as the $h$-th Fourier coefficient of the spectral density function. Conversely, the spectral density is the Fourier transform of the autocovariance function

$$
f(\omega)=\frac{1}{2 \pi} \sum_{h \in \mathbb{Z}} \gamma(h) e^{-\mathrm{i} h \omega},
$$

which is hermitian and non-negative definite. The autocovariance function and the spectral density function therefore form a Fourier pair. The spectral density, being 
the Fourier transform of the collection of autocovariance functions, thus encodes the entire second-order structure of the process.

The Spectral representation Theorem (Cramér, 1942), often called the Cramér representation, is as fundamental to spectral domain analysis as Wold's representation is to the time domain. It asserts that every zero-mean weakly stationary process can be represented as a superposition of sinusoids with random amplitudes and phases that are uncorrelated. An important ingredient in establishing the theorem is that the process $\left\{X_{t}: t \in \mathbb{Z}\right\}$ can be identified with a complex-valued stochastic process $\left\{Z_{\omega}:-\pi \leqslant \omega \leqslant \pi\right\}$. This is done by defining an isomorphic mapping $\mathcal{T}$ between subspaces $\overline{\mathrm{sp}}\left\{X_{t}\right\}_{t \in \mathbb{Z}}$ of $L^{2}(\Omega, \mathcal{A}, \mathbb{P})$ and $\overline{\mathrm{sp}}\left\{e^{\mathrm{it} \cdot}\right\}_{t \in \mathbb{Z}}$ of $L^{2}([-\pi, \pi], \mathscr{B}, F)$, where $F$ is the spectral measure of $\left\{X_{t}\right\}$. The process that is defined as

$$
Z_{\omega}=\mathcal{T}^{-1}\left(1_{(-\pi, \omega]}(\cdot)\right) \quad-\pi \leqslant \omega \leqslant \pi
$$

where $1_{(a, b]}(\cdot)$ denotes the indicator function on $(a, b]$, is a right-continuous complexvalued orthogonal increment process on $[-\pi, \pi]$. In particular, it satisfies

(i) $\mathbb{E} Z_{\omega}=0, \quad \omega \in[-\pi, \pi]$,

(ii) $\mathbb{E}\left|Z_{\omega}\right|^{2}<\infty, \quad \omega \in[-\pi, \pi]$,

(iii) $\mathbb{E}\left[\left(Z_{\omega_{4}}-Z_{\omega_{3}}\right) \overline{\left(Z_{\omega_{2}}-Z_{\omega_{1}}\right)}\right]=0, \quad\left(\omega_{1}, \omega_{2}\right] \cap\left(\omega_{3}, \omega_{4}\right]=\varnothing$.

The distribution function of the process $\left\{Z_{\omega}:-\pi \leqslant \omega \leqslant \pi\right\}$ is exactly the spectral measure $F$ of $\left\{X_{t}\right\}$ and its covariance structure satisfies

$$
\mathbb{E}\left|Z_{\omega}-Z_{\nu}\right|^{2}=F(\omega)-F(\nu), \quad-\pi \leqslant \nu \leqslant \omega \leqslant \pi .
$$

The Spectral Representation theorem then tells us that the elements of the process $\left\{X_{t}\right\}$ can be represented as the stochastic integral

$$
X_{t}=\int_{-\pi}^{\pi} e^{\mathrm{i} \omega t} d Z_{\omega}
$$

almost surely.

For the purpose of exposition, the previous results were introduced for univariate time series. The extension to $H=\mathbb{C}^{d}$ is straightforward because the results can be derived point-wise and then recombined without convergence problems. For $H=L^{2}([a, b], \mathscr{B}, \lambda)$, there are an infinite number of coordinates and the derivation of Bochner's Theorem is yet to be established. Existing literature in the functional setting is therefore restricted to weakly stationary functional time series that admit a so-called spectral density operator.

\section{Spectral analysis on the function space}

The generalization of spectral analysis for weakly stationary processes was first considered in Panaretos and Tavakoli (2013b). We state some of the main results here. Let $\left\{X_{t}: t \in \mathbb{Z}\right\}$ be a weakly stationary process taking values in $H=L_{\mathbb{R}}^{2}([0,1], \mathscr{B}, \lambda)$. In analogy to the finite-dimensional case, the autocovariance operator $\mathcal{C}_{h}$ can be 
shown to form a Fourier pair with an operator in the frequency domain given the dependence structure decays sufficiently fast. That is, if

$$
\sum_{h \in \mathbb{Z}}\left\|\mathcal{C}_{h}\right\|_{p}<\infty,
$$

then we can define the spectral density operator as its Fourier transform

$$
\frac{1}{2 \pi} \sum_{h \in \mathbb{Z}} \mathcal{F}_{\omega} e^{-\mathrm{i} h \omega},
$$

where the convergence holds in $\|\cdot\|_{p}$. The spectral density operator is self-adjoint, non-negative definite, $2 \pi$-periodic in $\omega$ and is an element of $S_{p}(H)$. Given the $X_{t}$ are elements of $\mathbb{H}$, the autocovariance operators and the spectral density operators are in $S_{2}(H)$. The interpretation of the spectral density operator is in complete analogy to the interpretation of spectral density matrix in the multivariate world in the following sense. Let $\left\{\left\langle X_{t}, \phi\right\rangle\right\}_{t \in \mathbb{Z}}$ and $\left\{\left\langle X_{t}, \psi\right\rangle\right\}_{t \in \mathbb{Z}}$ for given $\psi, \phi \in H$, then $\omega \mapsto\left\langle\mathcal{F}_{\omega}(\phi), \psi\right\rangle$ is in fact the cross-spectrum between the two series $\left\langle X_{t}, \phi\right\rangle_{t \in \mathbb{Z}}$ and $\left\langle X_{t}, \phi\right\rangle_{t \in \mathbb{Z}}$ and gives the the power spectrum in case $\phi=\psi$, where the equality is meant in an $L^{2}$ sense. Moreover, the autocovariance operator at lag $h$ can be represented as

$$
\mathcal{C}_{h}=\int_{-\pi}^{\pi} e^{\mathrm{i} h \omega} \mathcal{F}_{\omega} d \omega
$$

where the convergence holds in $\|\cdot \cdot\|_{p}$. The autocovariance operator and the spectral density operator can thus be viewed as generalization of the autocovariance matrix and spectral density matrix, respectively, to the functional setting.

Under the additional assumption that

$$
\sum_{h \in \mathbb{Z}}\left\|\mathcal{C}_{h}\right\|_{1}<\infty
$$

it has been shown (Panaretos and Tavakoli, 2013a) that a weakly stationary functional time series admits a representation in the spectral domain that is similar to the Cramér representation (1.1). An important aspect is again the identification of $\left\{X_{t}: t \in \mathbb{Z}\right\}$ with, in this case, a functional orthogonal increment process $\left\{Z_{\omega}:-\pi \leqslant \omega \leqslant \pi\right\}$. To establish this in the infinite-dimensional setting, denote $H^{\prime}$ the space of all measurable complex functions $y:[-\pi, \pi] \rightarrow \mathbb{C}$ such that

$$
\int_{-\pi}^{\pi}|y(\lambda)|^{2}\left\|\mathcal{F}_{\lambda}\right\|_{1} d \lambda<\infty
$$

where $\mathcal{F}$ is the spectral density operator of a weakly stationary process with elements $X_{t} \in \mathbb{H}$. Endowed with the inner product

$$
\int_{-\pi}^{\pi} x(\lambda) \overline{y(\lambda)}\left\|\mathcal{F}_{\lambda}\right\|_{1} d \lambda<\infty, \quad x, y \in H^{\prime}
$$

it forms a Hilbert space. Aforementioned authors established that there exists an isometric isomorphism $\mathcal{T}$ of $\overline{\mathrm{sp}}\left\{X_{t}\right\}_{t \in \mathbb{Z}}$ onto $H^{\prime}$ such that $\mathcal{T}$

$$
\mathcal{T} X_{t}=e^{\mathrm{i} t .} \quad t \in \mathbb{Z} .
$$


The process defined by $Z_{\omega}=\mathcal{T}^{-1}\left(1_{(-\pi, \omega]}(\cdot)\right)$ is then a functional orthogonal increment process of which the second-order properties are completely determined by the spectral density operator $\mathcal{F}$. We have

$$
\mathbb{E}\left[Z_{\omega_{2}}(\tau) \overline{Z_{\omega_{1}}(\sigma)}\right]=\int_{-\pi}^{\min \left(\omega_{1}, \omega_{2}\right)} f_{\lambda}(\tau, \sigma) d \lambda \quad \text { a.e. }
$$

Here, $f_{\lambda}$ is the kernel of $\mathcal{F}_{\lambda}$. In particular, since $\left\|\mathcal{F}_{\lambda}\right\|_{1}=\int_{0}^{1} f_{\lambda}(\tau, \tau) d \tau$ this implies

$$
\mathbb{E}\left\|Z_{\omega}\right\|_{2}^{2}=\int_{-\pi}^{\omega}\left\|\mathcal{F}_{\lambda}\right\|_{1} d \lambda
$$

A straightforward argument then shows that a weakly stationary functional time series has a functional Cramér representation

$$
X_{t}(\tau)=\int_{-\pi}^{\pi} e^{\mathrm{i} \omega t} d Z_{\omega}(\tau) \quad \tau \in[0,1] \quad \text { a.s. a.e. }
$$

where the stochastic integral is the Riemann-Stieltjes limit

$$
\lim _{J \rightarrow \infty} \sum_{j=1}^{J} e^{\mathrm{i} \omega_{j} t}\left(Z_{\omega_{j+1}}(\tau)-Z_{\omega_{j}}(\tau)\right) \quad \tau \in[0,1] \text { in } L^{2},
$$

for $-\pi=\omega_{1}<\ldots<\omega_{J+1}=\omega$ and $\max _{j=1, \ldots, J}\left|\omega_{j+1}-\omega_{j}\right| \rightarrow 0$ as $J \rightarrow \infty$.

\section{Estimation}

Because the main argument in this subsection applies both to the finite and infinite dimension, the rest of this subsection focuses on $H=L_{\mathbb{R}}^{2}([0,1], \mathscr{B}, \lambda)$. In practice, a stretch of length $T$ of the process $\left\{X_{t}: t \in \mathbb{Z}\right\}$ is observed. In order to draw inferences on the properties of spectral density operator of the process, a consistent estimator is required. The Spectral Representation Theorem shows that the infinitesimal increment $d Z_{\omega}$ has covariance operator $\mathcal{F}_{\omega}$. This observation leads to the consideration of stochastic properties of the functional discrete Fourier Transform (fDFT) of the sample $X_{0}, \ldots, X_{T-1}$, which is given by

$$
D_{\omega}^{(T)}(\tau)=(2 \pi T)^{-1 / 2} \sum_{t=0}^{T-1} X_{t}(\tau) e^{-\mathrm{i} \omega t} .
$$

This transform maps the $T$ real-valued random functions into a random element of $H=L_{\mathbb{C}}^{2}([0,1], \mathscr{B}, \lambda)$. We note that based on a stretch of length $T$, we can only identify $T$ distinct values of the fDFT sequence. In analogy to the finite-dimensional setting, the fDFT can be viewed as a rough estimator of $d Z_{\omega}$ and is therefore expected to have covariance that is close to the covariance operator $\mathcal{F}_{\omega}$. In fact it can be shown (Panaretos and Tavakoli, 2013a) that the sampled process $\left\{D_{\omega_{j}}\right\}_{j=0, \ldots, T-1}$, where $\omega_{j}=\frac{2 \pi j}{T}$ are the canonical frequencies, converge jointly to zero-mean Gaussian elements in $H=L_{\mathbb{C}}^{2}([0,1], \mathscr{B}, \lambda)$ that are asymptotically uncorrelated for $j_{1} \neq j_{2}$ 
and where $D_{\omega_{j}}$ has covariance operator $\mathcal{F}_{\omega_{j}}$. This is a property that will be exploited in Chapter 4 to build a frequency domain-based stationarity test.

As an estimator for the power spectrum $\mathcal{F}_{\omega}$, it is therefore natural to consider the periodogram operator given by

$$
I_{\omega}^{(T)}=\left[D_{\omega}^{(T)}\right] \otimes\left[D_{\omega}^{(T)}\right]^{\dagger}
$$

where $\uparrow$ denotes conjugate transposition. The original periodogram $(H=\mathbb{R})$ finds its origin in harmonic analysis, where it was used to look for hidden periodicities in the data. It was first developed by Schuster (1897), to determine if there was any hidden periodicity to the sunspot cycle. Sunspots are the dark spots on the surface of the sun which are visible from earth and caused by solar magnetic disturbances. Although the periodogram is an asymptotically unbiased estimator of the power spectrum, it has a variation that depends on the true spectrum and that does not disappear asymptotically. Given some weak dependence conditions are satisfied, the covariance structure of the periodogram kernel satisfies

$$
\begin{aligned}
\operatorname{cov}\left(I_{\omega_{1}}^{(T)}\left(\tau_{1}, \sigma_{1}\right), I_{\omega_{2}}^{(T)}\left(\tau_{2}, \sigma_{2}\right)\right)= & \frac{\sin ^{2}\left(\frac{T}{2}\left(\omega_{1}-\omega_{2}\right)\right.}{T^{2} \sin ^{2}\left(\frac{\omega_{1}-\omega_{2}}{2}\right)} f_{-\omega_{1}}\left(\tau_{1}, \tau_{2}\right) f_{-\omega_{1}}\left(\sigma_{1}, \sigma_{2}\right) \\
& +\frac{\sin ^{2}\left(\frac{T}{2}\left(\omega_{1}+\omega_{2}\right)\right.}{T^{2} \sin ^{2}\left(\frac{\omega_{1}+\omega_{2}}{2}\right)} f_{-\omega_{1}}\left(\tau_{1}, \sigma_{2}\right) f_{-\omega_{1}}\left(\sigma_{1}, \tau_{2}\right)+O\left(T^{-1}\right) .
\end{aligned}
$$

This expression shows that for $0 \leqslant \omega_{1} \neq \omega_{2}<\pi$, the periodogram kernel variates are asymptotically orthogonal. Because these variates are also continuous in $\omega$, it is expected that $I_{\omega_{1}}^{(T)}$ is close to $I_{\omega_{2}}^{(T)}$ when $\omega_{1}$ is close to $\omega_{2}$. This indicates that a reduction in variance can be achieved by local averaging the periodogram variates. A consistent estimator for the true spectral density operator can therefore be obtained by applying a linear smoothing filter that smooths the periodogram over neighboring frequencies. Such an estimator can be given by

$$
\hat{\mathcal{F}}_{\omega}=\int_{-\pi}^{\pi} \frac{1}{b_{\mathrm{t}, T}} K\left(\frac{\omega-\lambda}{b_{\mathrm{t}, T}}\right) I_{\lambda}^{(T)} d \lambda,
$$

where $K$ is a symmetric window function that determines the probability mass accorded to the various periodogram variates. The parameter $b_{\mathrm{t}, T}$ is the bandwidth and specifies the range of surrounding frequencies over which is averaged. While reducing the variance, application of the smoothing window leads to a loss of information on the dynamic behavior of the spectrum and thereby to an increase in bias. In order to control for this bias-variance trade-off, consistency requires the bandwidth should tend to zero at an appropriate rate as $T$ tends to infinity. Given this is satisfied, these nonparametric estimators $\hat{\mathcal{F}}_{\omega}$ can be shown to be consistent and moreover, asymptotically Gaussian estimators for the spectral density (operators) $\mathcal{F}_{\omega}$ at the corresponding frequencies.

Periodogram-based estimators suffer from spectral leakage. Spectral leakage is the phenomenon that energy located at a particular frequency will be partially leaked away to surrounding frequencies. This is caused by the underlying (functional) 
Discrete Fourier Transform which implicitly takes the input series to be periodic. If the stretch of data does not correspond to an integer multiple of its corresponding cycle, the waveform is truncated resulting in discontinuities. A data taper can mitigate this effect by smoothly weighing down the data at the endpoints to zero. The properties of the above estimator could therefore be improved by constructing the periodogram operator with tapered fDFT of the form

$$
D_{\omega}^{(T)}(\tau)=\left(2 \pi H_{2, T}\right)^{-1 / 2} \sum_{t=0}^{T-1} h_{t, T} X_{t}(\tau) e^{-\mathrm{i} \omega t}
$$

where $h_{\bullet, T}: \mathbb{R} \rightarrow \mathbb{R}^{+}$is a data taper of length $T$ and $H_{2, T}=\sum_{t=0}^{T-1} h_{t, T}^{2}$ the normalizing constant.

Alternative estimators for finite-dimensional time series have been proposed. For example, to take into account that the covariance structure depends on the unknown spectrum, an alternative could be based on the log-periodogram. However, without any structural modeling assumptions, a consistent estimator requires some form of smoothing and specification of a parameter that plays an equivalent role to the bandwidth parameter. Both the choice of the window function and bandwidth parameter affect the quality of the estimate but the choice of the bandwidth parameter is more important in accounting for the behavior of the estimate. Determining an optimal value for the bandwidth parameter is a well-known problem in nonparametric statistics and discrepancy measures, such as the integrated mean squared error, are widely used to find an optimal value. There exists an extensive literature on kernel spectral density estimation for finite-dimensional stationary processes. General discussions can for example be found in Brillinger (1981) and Brockwell and Davis (1991). In Chapter 2, a data-adaptive method is proposed for time-dependent spectra that can directly be applied to the estimation of spectra of weakly stationary processes.

\subsubsection{Time-varying spectral analysis}

In this section, a heuristic background is sketched for time-varying spectral analysis. Throughout focus is on univariate time series where the extension to multivariate finite dimensional time series is direct. Chapter 3 is involved with the generalization of time-varying spectral analysis to the infinite dimension. As discussed in the previous section, a process that can be represented as a superposition of sine and cosine waves with uncorrelated random coefficients and hence admits the representation

$$
X_{t}=\int_{-\pi}^{\pi} e^{\mathrm{i} \lambda t} d Z_{\lambda}
$$

is by definition weakly stationary. For nonstationary processes it is necessary to replace the functions $e^{\mathrm{i} \lambda t}$ with other kind of functions. It has been shown (see e.g. Grenander and Rosenblatt, 1957) that processes with autocovariance function

$$
c_{s, t}=E\left[X_{s} \bar{X}_{t}\right]=\int_{-\pi}^{\pi} \phi_{s}(\lambda) \phi_{t}^{\dagger}(\lambda) d \mu(\lambda),
$$


where the $\phi_{t}(\lambda)$ are square integrable with respect to the measure $\mu$ for each $t$, admit a representation

$$
X_{t}=\int_{-\pi}^{\pi} \phi_{t}(\lambda) d Z_{\lambda}
$$

with orthogonal process $\mathbb{E}\left|d Z_{\lambda}\right|^{2}=d \mu(\lambda)$. The role of the measure $\mu$ is analogous to the spectral measure $F$ for a weakly stationary process. In order to keep the concept of 'frequency' for nonstationary processes, Priestley (1965) introduced a class of nonstationary processes ${ }^{2}$ of which the functions $\phi_{t}(\lambda)$ have an oscillating form. Processes belonging to this class are called oscillating processes and can be represented as

$$
X_{t}=\int_{-\pi}^{\pi} A_{t}(\lambda) e^{\mathrm{i} \lambda t} d Z_{\lambda}
$$

where for each fixed $\lambda$, the transfer function $A_{t}(\lambda)$ is a slowly changing function of $t$ and has a generalized Fourier transform whose modulus has its absolute maximum at the origin. The representation (1.2) describes the temporal evolution of the spectral distribution of the process. More specifically, the evolutionary power spectrum at time $t$ with respect to the family of oscillatory functions $\left\{A_{t}(\lambda) e^{\mathrm{i} \lambda t}\right\}$ is defined as

$$
d F_{t}(\lambda)=\left|A_{t}(\lambda)\right|^{2} d \mu(\lambda)
$$

and if the measure is absolutely continuous with respect to $\lambda$, we have the timedependent spectral density at time $t$

$$
f(t, \lambda)=\left|A_{t}(\lambda)\right|^{2} \frac{d \mu}{d \lambda}
$$

The evolutionary spectrum keeps the same physical interpretation as the power spectrum in the weakly stationary case in the sense that it describes the energy distribution over frequency. However, the evolutionary spectrum describes the spectral content in the neighborhood of each time instant $t$, whereas the power spectrum gives the spectral content over all time $t$.

The class of oscillatory processes as introduced by Priestley (1965) contains weakly stationary processes as a special case (choosing $\phi_{t}(\lambda)=e^{\mathrm{i} \lambda t}$ ) and the notion of evolutionary spectra provides a generalization of the definition of power spectra. Yet, the approach of Priestley (1965) does not allow for a general meaningful asymptotic theory. To illustrate the problem, suppose we observe the process $\left\{X_{t}\right\}$ for $t=1, \ldots, T$ which has time domain representation

$$
X_{t}=a(t) X_{t-1}+\varepsilon_{t} \quad \varepsilon_{t} \stackrel{i . i . d .}{\sim} \mathcal{N}(0,1) \quad \forall t \in \mathbb{Z} .
$$

Inference on such a process requires inference on the parameter function $a(\cdot)$. Because the parameter curve varies over time, future observations do not necessarily contain any useful information on the values of $a(\cdot)$ on a given data segment. An

\footnotetext{
${ }^{2}$ Priestley (1965) focused on continuous time processes but the theory is the same for $t \in \mathbb{Z}$, the framework of this thesis.
} 
asymptotic approach where $T \rightarrow \infty$ is then not suitable to describe statistical inference procedures. In Dahlhaus (1996a), an alternative asymptotic theory was formulated for processes with evolutionary spectra. This theory is based on infill asymptotics, an approach in which one observes the process on an increasingly finer grid. The rough idea is that time is rescaled to the unit interval and hence that $a(t)$ is replaced with $a\left(\frac{t}{T}\right)$

$$
X_{t, T}=a\left(\frac{t}{T}\right) X_{t-1}+\varepsilon_{t} \quad t=1, \ldots, T .
$$

The resulting process $\left\{X_{t, T}\right\}$ forms a triangular array that is also depending on $T$. As $T$ increases, more observations at a local level are obtained. If the process is varying slowly over time, such an approach means we observe more and more observations of the structure of the same kind at a fixed time point as $T \rightarrow \infty$. This thus provides a framework for meaningful statistical inference. Dahlhaus (1996a) therefore studied processes that have a time-varying spectral representation

$$
X_{t, T}=\int_{-\pi}^{\pi} A_{t / T}(\lambda) e^{\mathrm{i} \lambda t} d Z_{\lambda} .
$$

It was shown that such a representation, with transfer function $A_{t / T}(\lambda)$ that changes smoothly over time, only holds approximately for time-varying autoregressive processes. This has led to the definition of the class of locally stationary processes. Processes belonging to this class admit a representation

$$
X_{t, T}=\int_{-\pi}^{\pi} A_{t, T}(\lambda) e^{\mathrm{i} \lambda t} d Z_{\lambda}
$$

where the transfer function $A_{t, T}(\lambda)$ closely approximates the function $A_{t / T}(\lambda)$, which is smooth in time direction. More generally, a process is called locally stationary if in the neighborhood of each time point $u=t / T \in[0,1]$, there exists a weakly stationary process $\left\{X_{t}^{(u)}\right\}$, such that

$$
\left|X_{t, T}-X_{t}^{(u)}\right|=O_{p}\left(\left|\frac{t}{T}-u\right|+\frac{1}{T}\right) .
$$

A detailed example as well as the precise definition for time series and functional time series of this class are given in Chapter 2 and 3, respectively. The time-varying spectrum of a locally stationary process $\left\{X_{t, T}\right\}$ is unique and is locally the same as the spectral density $f(u, \lambda)$ of the process $\left\{X_{t}^{(u)}\right\}$. If $\left\{X_{t, T}\right\}$ admits a time-varying spectral representation as in (1.3), the time-varying spectrum is of the form

$$
f(u, \lambda)=\frac{1}{2 \pi}\left|A_{u}(\lambda)\right|^{2} .
$$

The time-varying spectral density forms a Fourier pair with the function $\gamma(u, \cdot)$, where

$$
\gamma(u, h)=\int_{-\pi}^{\pi} f(u, \lambda) e^{i \lambda h} d \lambda, \quad h \in \mathbb{Z} .
$$

Since $\operatorname{cov}\left(X_{\lfloor u T\rfloor, T}, X_{\lfloor u T\rfloor+h, T}\right)=\gamma(u, h)+O\left(\frac{1}{T}\right)$ the function $\gamma(u, h)$ is called the local autocovariance function of the process $\left\{X_{t, T}\right\}$ at time point $u=t / T$. 


\section{Estimation}

This section briefly discusses estimation of the time-varying spectral density. Suppose a sample of a zero-mean locally stationary process $X_{1, T}, \ldots X_{T, T}$ is observed. Because the process is locally stationary and hence approximately stationary on a small enough data-segment, say of length $N_{T}$, statistical methods developed for weakly stationary processes can be applied on this segment. Estimation can therefore be based on the segmented or short-time periodogram

$$
I_{T}(u, \omega)=\frac{1}{2 \pi H_{2, N_{T}}}\left|\sum_{s=0}^{N_{T}-1} h_{s, N_{T}} X_{\lfloor u T\rfloor-N_{T} / 2+s+1, T} e^{-\mathrm{i} \omega s}\right|^{2}
$$

where $h_{(\bullet), N_{T}}$ is a data taper of length $N_{T}$ and $H_{2, N_{T}}=\sum_{s=0}^{N_{T}-1} h_{s, N}^{2}$ the normalizing constant. The use of a data taper is in the locally stationary case especially important to improve estimation. Not only will it reduce bias due to spectral leakage but the application of a data taper can moreover control the bias that stems from the degree by which $X_{t, T}$ departs from $X_{t}^{(u)}$. The data taper plays in fact an equivalent role (see Chapter 3) to a smoothing window in time direction with bandwidth parameter $b_{\mathrm{t}, T}=N_{T} / T$. Just as in the weakly stationary case, the segmented periodogram $I_{T}(u, \omega)$ is not consistent and needs to be convolved with a smoothing window in frequency direction. That is,

$$
\hat{f}_{T}(u, \omega)=\int_{-\pi}^{\pi} \frac{1}{b_{\mathrm{f}, T}} K_{\mathrm{f}}\left(\frac{\omega-\lambda}{b_{\mathrm{f}, T}}\right) I_{T}(u, \lambda) d \lambda
$$

where $K_{\mathrm{f}}$ is the smoothing window and $b_{\mathrm{f}, T}$ is the bandwidth parameter.

An alternative estimator can be based on a localized version of the periodogram, the pre-periodogram. The pre-periodogram was first introduced in Neumann and von Sachs (1997) as a starting point for a wavelet-based estimator of the timevarying spectral density. The pre-periodogram is given by

$$
J_{T}(u, \lambda)=\frac{1}{2 \pi} \sum_{k: 1 \leqslant\left\lfloor u T+\frac{1}{2} \pm \frac{k}{2}\right\rfloor \leqslant T} X_{\left\lfloor u T+\frac{k+1}{2}\right\rfloor, T} X_{\left\lfloor u T-\frac{k-1}{2}\right\rfloor, T} e^{-\mathrm{i} k \lambda}
$$

and is discussed in detail in Chapter 2. To obtain a consistent estimator, the preperiodogram requires convolution with smoothing windows in both frequency and time direction and is of the form

$$
\hat{f}_{T}(u, \omega)=\int_{0}^{1} \int_{-\pi}^{\pi} \frac{1}{b_{\mathrm{t}, T}} K_{\mathrm{t}}\left(\frac{u-v}{b_{\mathrm{t}, T}}\right) \frac{1}{b_{\mathrm{f}, T}} K_{\mathrm{f}}\left(\frac{\omega-\lambda}{b_{\mathrm{f}, T}}\right) J_{T}(u, \lambda) d \lambda d v .
$$

Here $K_{\mathrm{t}}$ and $b_{\mathrm{t}, T}$ denote the smoothing window and bandwidth parameter in time direction. As explained in Chapter 2, the latter estimator provides more flexibility in adjusting the degree of smoothing in either direction. Under suitable regularity conditions, the estimators (1.4) and (1.5) are asymptotically equivalent. In particular, 
the first two moments satisfy

$$
\begin{aligned}
& \mathbb{E} \hat{f}_{T}(u, \omega)=f(u, \omega)+ \frac{1}{2} b_{\mathrm{t}, T}^{2} \int x^{2} K_{\mathrm{t}}(x) d x \frac{\partial^{2} f(u, \omega)}{\partial u^{2}}+\frac{1}{2} b_{\mathrm{f}, T}^{2} \int x^{2} K_{\mathrm{f}}(x) d x \frac{\partial^{2} f(u, \omega)}{\partial \omega^{2}} \\
&+o\left(b_{\mathrm{t}, T}^{2}+b_{\mathrm{f}, T}^{2}+\frac{\log b_{\mathrm{t}, T} T}{b_{\mathrm{t}, T} T}\right), \\
& \operatorname{var} \hat{f}_{T}(u, \omega)=\left(b_{\mathrm{f}, T} b_{\mathrm{t}, T} T\right)^{-1} 2 \pi f^{2}(u, \omega) \int K_{\mathrm{f}}^{2}(x) d x \int K_{\mathrm{t}}^{2}(x) d x\left(1+\delta_{\lambda, 0}\right) .
\end{aligned}
$$

Compared to the weakly stationary case, the bias-variance trade-off is in the locally stationary case additionally affected by the degree of nonstationarity on the segment. This bias needs to be balanced with the bias term that is due to variation in frequency direction and with the variance term. If the degree of nonstationarity is small then more smoothing should be put in time direction, while little variation of the spectrum in frequency direction implies more smoothing should be put in frequency direction. In particular, consistency requires that $b_{\mathrm{f}, T} \rightarrow 0$ and $b_{\mathrm{t}, T} \rightarrow 0 \mathrm{such}$ that $b_{\mathrm{f}, T} b_{\mathrm{t}, T} T \rightarrow \infty$ as $T \rightarrow \infty$. The optimal bandwidths were derived in Dahlhaus (1996b) and depend both (inversely) on the partial second-order derivatives in time and frequency direction of the true unknown time-varying spectrum. The sensitivity of the estimation performance to the choice of the bandwidth parameters has motivated the data-adaptive approach introduced in the next chapter.

\subsubsection{The contribution of this thesis}

This section provides a short overview of the three subsequent chapters, which form the main contribution of this thesis. The first part of this thesis is devoted to address a long-time open practical problem in the analysis of time-varying spectra, while the second part of this thesis is devoted to fill the gap in theory and methodology for the analysis of functional time series with time-varying spectral characteristics. The individual contributions of the respective chapters are discussed in turn.

Chapter 2 is devoted to the development of a data-adaptive procedure to estimate the spectral density of nonstationary processes. The type of nonstationarity can be caused by both slowly changing spectral characteristics as well as structural breaks. The approach is motivated by the dependence, as mentioned in the previous subsection, of the optimal bandwidths on the smoothness properties of the unknown spectrum. In practice, these are not directly applicable and the estimation performance depends considerably on the localization in time and frequency direction. A data-adaptive choice to optimally select the bandwidths has so far remained an open problem.

The objective of Chapter 2 is to address this problem and to introduce an approach that allows to determine the shape of the smoothing kernel - viewed as a bivariate kernel in the time-frequency plane - in a data-adaptive manner. The underlying idea of the algorithm is to iteratively extend the smoothing neighborhood until 'homogeneity' becomes violated. Homogeneity refers to the property that the spectral variates are independent and identically distributed within a given neighborhood of the time-frequency plane. This separation is achieved by means 
of a penalty kernel that penalizes regions not considered homogeneous. In order the permit full flexibility for the degree of smoothing in time and frequency direction, estimates of the local spectra are obtained by applying the adaptive smoothing kernels to the pre-periodogram. This allows to obtain both good temporal and frequency resolution simultaneously, something that is more difficult with segmented periodogram-based estimators. The flexibility that the pre-periodogram offers as an underlying raw estimator comes however at the expense of artifacts such as the introduction of negative values. A particular feature of the algorithm, which is integrated in an additional stability step, is to mitigate this issue. A simulation study is performed to analyze the performance of the algorithm. The method is then applied to neuroscientific data and, in particular, to Local Field Potential recordings.

Many natural phenomena vary over a continuum but only recent technological improvements have made it possible to take measurement of such processes at arbitrarily high frequency. The resulting increasing demand for methodology that allows to extract information and to draw inferences from data that can be considered as being sampled recordings from complex mathematical structures such as images, curves or surfaces, is the prime focus of functional data analysis. Most of the functional data analysis literature assumes independent and identically distributed functional observations. Just as for stochastic processes on finite-dimensional Hilbert spaces, it is important for time series of functional data to develop methods that not only take into account the dependence structure but also that this structure might change over time. So far, statistical methods and tools for functional time series have only been derived under the assumption of weak stationarity. The main implications of the violation of this assumption, which were discussed at the start of this Introduction, provide the motivation for the research conducted in Chapter 3 and Chapter 4.

The need to relax the assumption of weak stationarity for functional time series is addressed in Chapter 3. A theoretical framework is introduced that enables to draw statistical inferences from time series of functional data of which the dynamics change over time. The concept of local stationarity is put forward for functional time series and a spectral theory is developed. That is, a time-varying functional Cramér representation is derived and a class of processes is established that can be defined by means of this representation. This class is shown to satisfy the definition of functional local stationarity. The notion of a time-varying spectral density operator is introduced and its properties are derived. In analogy to the multivariate world, a uniqueness result can be obtained. Time-varying functional ARMA processes are examined in detail and shown to admit a well-defined time-varying functional spectral representation. In the analysis, a distinction is made between transfer operators - the generalization of the Fourier Transform of the sequence of moving average matrices - that are bounded in operator norm and those that belong to the subclass of Hilbert-Schmidt operators. The framework is then used to construct an estimator of the spectral density operator based on a functional version of the segmented periodogram matrix. In particular, its consistency and asymptotic distribution are studied. 
When the assumption of weak stationarity is violated, the application of most existing statistical inference procedures for functional time series is inappropriate. This can result in severe model misspecification and consequently to incorrect inferences. It is therefore important to develop a suitable test that allows to determine whether the assumption of temporal constancy is legitimate. Chapter 4 is devoted to introduce a spectral domain-based method to test for weak stationarity of a functional time series. It exploits that the functional Discrete Fourier Transform at distinct frequencies is asymptotically uncorrelated if and only if the process is weakly stationary. The test statistic is based on an empirical covariance operator of the Discrete Fourier Transforms, which is subsequently projected. The methodology is theoretically justified through asymptotic results. The asymptotic properties of the statistic are derived under the null of functional weak stationarity and under the smooth alternative of functional local stationarity. Chapter 4 therefore builds on the asymptotic framework that was introduced in Chapter 3. No structural modeling assumptions are made other than functional versions of cumulant mixing conditions. A simulation study is performed to investigate the performance of the test in finite samples. 



\section{Chapter 2}

\section{Data-adaptive estimation of time-varying spectral densities}

\subsection{Introduction}

Spectral analysis of time series data has been of interest for many years and has a varied history owing to applications in a wide range of disciplines such as geophysics, astronomy, sound analysis, analysis of medical data or yet of economical data. There exists a rather extensive literature on spectral analysis of weakly stationary processes and statistical techniques are well developed (Cramér, 1942; Bartlett, 1950; Grenander and Rosenblatt, 1957; Cooley and Tukey, 1965; Brillinger, 1981). However, in most aforementioned applications the time series show nonstationary behavior and imposing weak stationarity is too restrictive. Especially when data is measured over longer periods of time, it is more plausible that the dynamics of the process change thereby invalidating the stationarity assumption. For this reason, the analysis of nonstationary time series has received more attention over the years.

Despite of its importance, the extension of existing estimation methods to processes that are not generated by time-invariant mechanisms is not a natural one. Classical asymptotic theory is by definition conflicting with the idea that the underlying probabilistic structure of the process changes over time. Two main approaches that deal with time-varying spectra are provided in the literature. In one approach, it is assumed that signals are subject to structural breaks and can be considered piecewise stationary. For example, Ombao et al. (2001) introduce a method to automatically split the signal into piecewise stationarity segments and select the span of SLEX (Smooth Localised Complex Exponentials) basis functions used to obtain smoothed estimates of the time-varying spectrum. Another approach, which was first considered by Priestley (1965), is to assume the underlying dynamics change slowly such that a smooth time-dependent spectrum exists and, at a local level, the process is approximately stationary. The locally stationary setting of Dahlhaus (1996a) provides a framework for meaningful inference of these type of processes and comprises weak stationarity as a special case. We will adopt the latter theoretical framework as to include applications that exhibit slowly changing behavior. At the 
same time our procedure will automatically adjust to possible structural breaks in the spectrum.

A well-known difficulty for inference on the latter type of processes is the necessity of local estimates of the time-dependent spectrum which requires kernel smoothing at a local level. As for nonparametric estimation in general, inherent to these methods is the issue of bandwidth selection. Although this problem also arises in the stationary case where a bandwidth in frequency direction needs to be set, the time-varying case requires additionally specification of a bandwidth in time direction. Time-dependent spectral density estimation becomes therefore a twodimensional problem in which one needs to be able to adjust for possible different degrees of smoothness of the spectrum in both directions. Proper adjustment is important in practice since the accuracy of corresponding estimates has been shown to be quite sensitive to the choice of smoothing bandwidths in both time as well as frequency direction. See for example Eichler et al. (2011), who investigate principal component analysis in the frequency domain for time-varying dynamic factor models. Theoretical optimal bandwidths depend on the unknown underlying spectrum and no guidelines are available on how to set them in practice (Dahlhaus, 2009). To our knowledge, data-adaptive schemes have not yet been considered. Under specific parametric assumptions there are however methods available based on the segmented periodogram. For example, Sergides and Paparoditis (2009) and Preuß et al. (2011) use an integrated version to test for semi-parametric hypotheses which avoids bandwidth selection in frequency direction. The major drawback of such underlying estimate is that a fixed time bandwidth must be set which complicates, for example, detecting a possible break in the spectrum.

The objective of this chapter is to construct local spectral density estimates where the respective smoothing kernels are adapted to the data at hand. This approach circumvents the problem of optimal bandwidth selection in the strict sense and permits full flexibility for the degree of smoothing. The procedure is based on the propagation-separation approach introduced by Polzehl and Spokoiny (2006) who investigate local likelihood estimation of exponential family models. The general idea behind it is to determine a maximal local neighborhood of a design point $f_{i}$ such that some local parametric assumption is still justified by the data. One starts with a small local neighborhood to estimate the parameter value and, during each step, the neighborhood is extended to include new data points for which the parametric assumption is not violated. This property is referred to as propagation. At the same time, points for which the assumption is violated are not included, i.e., areas will be separated in that case.

We introduce a bivariate propagation-separation approach to estimate the structure of the time-dependent spectrum of nonstationary time series over the timefrequency plane $U \times \Pi:=[0,1] \times[-\pi \times \pi]$. For each design point in the plane, a maximal local neighborhood is determined iteratively over which smoothing is justified by the data. More specifically, the neighborhood used for estimation in step $k$ of a local spectral variate $f\left(u_{l}, \lambda_{i}\right)$ will be described by a set of weights $\left\{W_{l, i}^{(k)}(s, j)\right\}$ that defines the shape of the kernel and is derived using the spectral estimates constructed at the previous step, $k-1$. The effective neighborhood over 
which is smoothed for estimation in step $k$ is then given by the points for which the corresponding weights from step $k-1$ are significantly different from zero. The big advantage is that the various design points are allowed to have kernels that are constructed with different weights functions leading to full adjustability in terms of shape and effective bandwidths. This is especially important in the presence of peaks or breaks for which a smaller effective bandwidth will reduce the problem of over-smoothing. At the same time, we will be able to get more accuracy in stable areas as the effective bandwidth is permitted to be relatively larger than would a global bandwidth have been set. It is worth remarking that the smoothing kernels can in fact be asymmetric. In particular, this feature of the method enables automatic adjustment to the presence of breaks in the spectrum. It should moreover be pointed out that the extension to the frequency domain is not obvious as the assumptions underlying the case of Polzehl and Spokoiny (2006) do not hold nor do we apply the principle of propagation-separation in the same manner.

This chapter is organized as follows. In section 2, we describe the type of processes of interest and introduce the building blocks of the estimator. Then, in section 3, the procedure is discussed while section 4 describes the effect of the choice of parameters and general guidelines are given. In section 5 , the properties of the approach are illustrated by application to simulated data. In section 6 , the approach is applied to Local Field Potential recordings.

\subsection{Theoretical framework: Locally stationary pro- cesses}

Before we introduce the procedure, we first discuss what we mean by time-dependent spectra and how they can be estimated. Since there is no time-dependent spectrum that is linearly connected to the covariance function of the process and at the same time keeps all nice underlying properties that exist in the weakly stationary case, different definitions for time-dependent spectra have been provided in the literature (e.g. Priestley, 1965; Subba Rao, 1970; Martin and Flandrin, 1985; Hallin, 1986). Most definitions depend in the classical way on $T$, the length of the time series, and therefore do not provide a meaningful asymptotic framework as future observations will generally not provide any relevant information of the probabilistic structure over the observed time period. In order to exploit large sample properties at a local level, we shall adhere to the theoretical framework of locally stationary processes as introduced by Dahlhaus (1996a) which is based on infill asymptotics. Instead of considering the same process for larger values of $T$, the idea of this approach is to redefine the process in such a way that one observes the parameter curves on a finer grid so that a more and more dense design is obtained as $T$ tends to infinity. For example, for a time-varying autoregressive process of order 1, this leads to a sequence of processes $\left\{X_{t, T}\right\}$

$$
X_{t, T}=a\left(\frac{t}{T}\right) X_{t-1, T}+\sigma\left(\frac{t}{T}\right) \varepsilon_{t}, \quad t=1, . ., T, T \in \mathbb{N},
$$


where $a(\cdot)$ and $\sigma(\cdot)$ are real-valued continuous functions on $[0,1]$. The time-varying parameters of $X_{t, T}$ depend then also on $T$ such that for increasing $T$ one obtains more and more observations with approximately the same dynamic dependence. Thus such families of processes provide a framework for asymptotic analysis permitting the researcher to consider properties of estimators such as consistency or asymptotic normality. We emphasize that this framework is purely theoretical as in practice $T$ is fixed and increasing $T$ will lead to considering a different time series. Put differently, if we increase $T$, we consider a different 'level' in the family than that we have data for. This discrepancy should be kept in mind when applying asymptotic results in practice to approximate those for finite $T$. Processes that can be analyzed by this framework must be locally approximately stationary in the sense that over short time periods they can be well approximated by a stationary process while their characteristics such as the covariance structure and parameters change gradually over longer time periods. More specifically, we assume (Dahlhaus, 1996a):

Definition 2.2.1 (Local stationarity). A sequence of stochastic processes $\left\{X_{t, T}, t=\right.$ $1, . ., T, T \in \mathbb{N}\}$ is called locally stationary with transfer function $A^{\circ}$ and mean function $\mu$ if there exists a representation

$$
X_{t, T}=\mu\left(\frac{t}{T}\right)+\int_{-\pi}^{\pi} e^{\mathrm{i} \lambda t} A_{t, T}^{\circ}(\lambda) d \xi(\lambda)
$$

with the following properties:

(i) $\xi(\lambda)$ is an orthogonal increment process defined on $[-\pi, \pi]$ with $\overline{\xi(\lambda)}=\xi(-\lambda)$, $\mathbb{E}\left(\xi_{a}(\lambda)\right)=0$ such that

$$
\operatorname{cum}\left(d \xi\left(\lambda_{1}\right), . ., d \xi\left(\lambda_{k}\right)\right)=\eta\left(\sum_{j=1}^{k} \lambda_{j}\right) h_{k}\left(\lambda_{1}, . ., \lambda_{k-1}\right) d \lambda_{1}, . ., d \lambda_{k}
$$

where $\operatorname{cum}(\cdot)$ denotes the $k$-th order cumulant, $h_{k}$ are cumulant densities with $h_{1}=0, h_{2}(\lambda)=1$, and $\left|h_{k}\left(\lambda_{1}, . ., \lambda_{k-1}\right)\right| \leqslant C_{k}$ for all $k \in \mathbb{N}$, and $\eta(\lambda)=$ $\sum_{j \in \mathbb{Z}} \delta(\lambda+2 \pi j)$ is the $2 \pi$-periodic extension of the Dirac delta function.

(ii) There exist a constant $C$ and a $2 \pi$-periodic smooth function $A:[0,1] \times \mathbb{R} \rightarrow \mathbb{C}$ with $\overline{A(u, \lambda)}=A(u,-\lambda)$ such that

$$
\sup _{t, \lambda}\left|A_{t, T}^{\circ}(\lambda)-A\left(\frac{t}{T}, \lambda\right)\right| \leqslant \frac{C}{T}
$$

for all $T \in \mathbb{N}$.

With this spectral representation, the time-varying spectral density of the process $\left\{X_{t, T}\right\}$ is defined as

$$
f(u, \lambda)=\frac{1}{2 \pi} A(u, \lambda) A(u, \lambda)^{*}
$$

for $u \in[0,1]$ and $\lambda \in[-\pi, \pi]$. If the transfer function $A(u, \lambda)$ is smooth, $f(u, \lambda)$ is the mean square limit of the Wigner-Ville spectrum (Martin and Flandrin, 1985)

$$
f_{T}(u, \lambda)=\frac{1}{2 \pi} \sum_{s=-\infty}^{\infty} \operatorname{cov}\left(X_{\lfloor u T-s / 2\rfloor, T}, X_{\lfloor u T+s / 2\rfloor, T}\right) e^{-\mathrm{i} s \lambda},
$$


where the processes $X_{t, T}$ are extended on $\mathbb{Z}$ by setting $A_{t, T}^{\circ}(\lambda)=A_{1, T}^{\circ}(\lambda)$ for $t<1$ and $A_{t, T}^{\circ}(\lambda)=A_{T, T}^{\circ}(\lambda)$ for $t>T$. As explained in Dahlhaus (1996a), the necessity of defining both $A_{t, T}^{\circ}(\lambda)$ and $A(u, \lambda)$ comes from the fact that for certain processes the spectral representation in (2.2) holds but the representation with a smooth common filter in continuous time for all $T$ only holds approximately. The smoothness of the latter is however necessary to derive distributional properties. An important consequence of this approach, and something that is not possible in non-rescaled time for time-varying processes, is the uniqueness of the time-varying spectral density.

Since our interest lies in estimation of smooth time-varying spectral densities with possible breaks in the spectrum, we moreover assume the following condition holds:

Assumption 2.2.2. $\left\{X_{t, T}, t=1, . ., T, T \in \mathbb{N}\right\}$ is defined as in definition 2.2.1 and has spectral density $f(u, \lambda)$ that is twice differentiable in $\lambda$ and piecewise twice differentiable in $u$ with bounded derivatives in both directions.

Throughout this chapter, rescaled time on the unit interval $\frac{t}{T}$ will be denoted by $u$. Furthermore, for sake of simplicity we assume that the mean function $\mu$ is zero.

\subsection{Propagation-separation approach in the time- frequency plane}

Obtaining consistent estimates of the local time--varying spectrum requires smoothing in both time and frequency direction of some underlying raw estimator. Although various asymptotic results are available (see e.g. Dahlhaus, 2009; Dahlhaus and Polonik, 2009), determining the corresponding optimal smoothing bandwidths in practice is still an open problem. The purpose of this chapter is to data-adaptively determine the shape of the smoothing kernels, which are then consequently used to obtain estimates of the local spectral variates. We start with small fixed bandwidths to obtain initial estimates. Then, at each iteration, the neighborhood used for estimation is extended in the direction for which the assumption of 'homogeneity' is still satisfied. We stop smoothing in those directions where it gets violated. This is achieved by means of a penalty kernel that penalizes regions not considered homogeneous.

\subsubsection{Example}

To capture the main idea, imagine the simple case where we have a white noise signal with a break in time direction at point $u_{b}$ as given in Figure 2.1 (a). It should be clear that for point $u_{1}$, we do not want to smooth over point $u_{2}$, which lies on the other side of the break. On the other hand, we would like to smooth over as many data points on the left side of the break for the construction of the kernel of point $u_{1}$ as the process is clearly stationary there. Ideally, the kernel should thus be constructed in such a way that it smooths as much as possible over the points on the left of the 
break but that gets cut off once we hit the break point. Our method allows for this by, in each iteration, comparing the data points in the extended neighborhood with each other in the so-called penalty step. Further smoothing only occurs if the data points are considered 'similar' by this step. For point $u_{1}$, we should therefore obtain a kernel as given in Figure 2.1 (b).

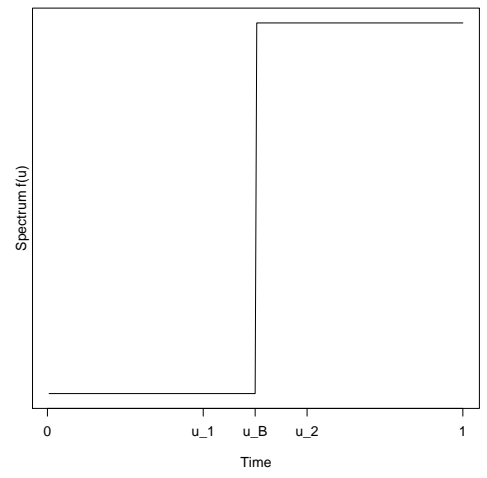

(a) Flat spectrum with break

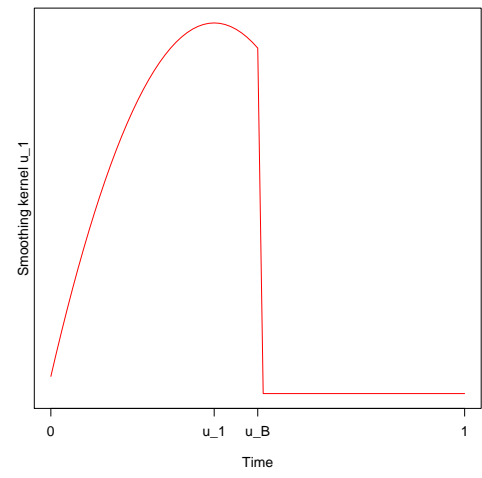

(b) Ideal smoothing kernel at $u_{1}$

Figure 2.1

A simplified version of the algorithm is as follows:

1. Initialisation: Construct initial estimates $\hat{f}_{T}^{\mathrm{in}}(u, \lambda)$ for all points in the timefrequency plane using small initial bandwidths $b_{\mathrm{t}, T}^{(0)}$ and $b_{\mathrm{f}, T}^{(0)}$ in time and frequency direction, respectively.

2. Penalty step: For each point, compare the estimate with all other estimates in the initial smoothing neighborhood. If a point in this neighborhood is deemed 'too different', then that particular point receives less relative weight in construction of the adaptive kernel for the point of interest.

3. Memory step: Control for too fast changes and cross-interference terms by comparing the current with the previous estimate. Set $k=1$.

4. Increase searching bandwidth to $b_{\mathrm{t}, T}^{(k}, b_{\mathrm{f}, T}^{(k)}$ and repeat penalty and memory step on the new estimates. Set $k=k+1$ and repeat until $k=k_{\max }$.

Before we discuss the algorithm in more detail, it should be clear that we need a couple of elements. Firstly, we require an underlying raw estimator to construct initial estimates. Secondly, we need to construct the adaptive estimator which is based on the penalty step. To allow for gradual changes in the spectrum and overall stability, we additionally need a memory step. We shall discuss these in turn.

\subsubsection{A local estimator: the modified pre-periodogram}

As an underlying raw estimator, most of the literature considers a segmented periodogram formed as a weighted average of fixed possibly overlapping time segments 
(e.g. Dahlhaus, 1996a; Preuß et al., 2011). The optimal segment length, which should be set explicitly, is directly depending on the relation of smoothness in time and frequency direction; a higher time resolution requires a shorter segment length but will yield a lower frequency resolution and vice versa. Another possibility is to use the pre-periodogram which is a localized version of the periodogram and was first introduced by Neumann and von Sachs (1997) who considered it for estimation of time-varying spectral densities by means of wavelets. Just like for the periodogram, the uncertainty principle still implies that the accuracy of estimation is limited by the reciprocal relationship that exists between time and frequency resolution. The advantage of the pre-periodogram over the periodogram is however that the degree of smoothing in both time and frequency direction is left to the smoothing step and therefore gives more freedom in adjusting for either direction based on local behavior of the observed process. Especially in our case, this is useful as we would like to adjust the kernel adaptively. For this reason, we shall use the pre-periodogram as underlying raw estimator. It is given by

$$
J_{T}(u, \lambda)=\frac{1}{2 \pi} \sum_{k: 1 \leqslant\left\lfloor u T+\frac{1}{2} \pm \frac{k}{2}\right\rfloor \leqslant T} X_{\left\lfloor u T+\frac{k+1}{2}\right\rfloor, T} X_{\left\lfloor u T-\frac{k-1}{2}\right\rfloor, T} e^{-\mathrm{i} k \lambda} .
$$

Here, the term $X_{\left\lfloor u T+\frac{k+1}{2}\right\rfloor, T} X_{\left\lfloor u T-\frac{k-1}{2}\right\rfloor, T}$ can be regarded as a preliminary estimator of the time-varying covariance function at rescaled time $u$ and lag $k$, which is defined as the inverse Fourier transform of the time-varying spectral density,

$$
\gamma(u, k)=\int_{-\pi}^{\pi} f(u, \lambda) e^{\mathrm{i} \lambda k} d \lambda
$$

The ordinary periodogram can be recovered by averaging the pre-periodogram variates over $t=1, \ldots, T$. Its expectation for fixed $T$ is moreover easily seen to coincide with the Wigner-Ville spectrum (2.5). If averaging is not done over all data points $t$, these type of preliminary estimators (Martin and Flandrin, 1985) will suffer from the presence of cross-interference terms. This leads to bad behavior such as possible negative estimates. We tailored our method to be able to cope with this. As a first step, we use a slightly modified definition of the pre-periodogram as a preliminary estimator. For odd lags, the classical pre-periodogram shows an asymmetry about the central point $t=u T$. Cross-interference terms can be dampened by using an alternative symmetric definition that would place the product exactly at the midpoint $\frac{t+s}{2}$. For $k=|t-s|$ odd, this is however not in line with one of the observations. We can take care of this by letting the preliminary covariance estimator average two neighboring covariances (in time) if no exact estimator is available. The idea is therefore to define the preliminary covariance estimator by

$$
C^{\star}(t, k)= \begin{cases}X_{t-\frac{k}{2}} X_{t+\frac{k}{2}} & \text { if } t \pm \frac{k}{2} \in \mathbb{Z}, \\ \frac{1}{2}\left(X_{t-\frac{k-1}{2}} X_{t+\frac{k-1}{2}}+X_{t+\frac{k+1}{2}} X_{\left.t+\frac{k+1}{2}\right)}\right. & \text { otherwise }\end{cases}
$$

at time points $t$ such that $t \in\left\{1, \frac{3}{2}, 2, \frac{5}{2}, \ldots, T-1, T-\frac{1}{2}, T\right\}$ and $0 \leqslant u \leqslant \min \{2(t-$ $1) / T, 2(T-1) / T\}$. Outside the specified range, the estimator is set to zero. With 
this definition, the pre-periodogram now becomes

$$
J_{T}(u, \lambda)=\frac{1}{2 \pi} \sum_{k=-(T-1)}^{T-1} C^{\star}(t, k) e^{-\mathrm{i} k \lambda}
$$

for time points $u \in\left\{\frac{2}{2 T}, \frac{3}{2 T}, \ldots 1\right\}$.

The pre-periodogram is asymptotically unbiased for the time-varying spectrum $f(u, \lambda)$. However, it is not consistent as its variance asymptotically diverges to infinity. A consistent estimator can be obtained by convolving the pre-periodogram with kernel functions in both directions, i.e.,

$$
\hat{f}_{T}(u, \lambda)=\frac{1}{b_{\mathrm{f}, T} b_{\mathrm{t}, T}} \int_{0}^{1} \int_{-\pi}^{\pi} K_{\mathrm{f}}\left(\frac{\lambda-\mu}{b_{\mathrm{f}, T}}\right) K_{\mathrm{t}}\left(\frac{u-v}{b_{\mathrm{t}, T}}\right) J_{T}(v, \mu) d \mu d v,
$$

where $K_{\mathrm{f}}$ and $K_{\mathrm{t}}$ denote the kernels for smoothing in frequency- and in time direction, respectively, and $b_{\mathrm{f}, T}$ and $b_{\mathrm{t}, T}$ are the corresponding bandwidths. The bandwidths should tend to zero at an appropriate rate as $T \rightarrow \infty$. In practice, smoothing of the pre-periodogram is based on a discretized version of the above kernel estimator

$$
\hat{f}_{T}(u, \lambda)=\frac{1}{C} \sum_{s, j} K_{\mathrm{f}}\left(\frac{\lambda-\lambda_{j}}{b_{\mathrm{f}, T}}\right) K_{\mathrm{t}}\left(\frac{u-s / T}{b_{\mathrm{t}, T}}\right) J_{T}\left(\frac{s}{T}, \lambda_{j}\right),
$$

where $\lambda_{j}=\frac{\pi j}{T}$ for $j=1-T, \ldots, T$ denote the Fourier frequencies and $C=$ $\sum_{s, j} K_{\mathrm{f}}\left(\left(\lambda-\lambda_{j}\right) / b_{\mathrm{f}, T}\right) K_{\mathrm{t}}\left((u-s / T) / b_{\mathrm{t}, T}\right)$ is the normalization constant. The properties of (2.11) have been investigated in the setting of empirical spectral processes in Dahlhaus (2009) and Dahlhaus and Polonik (2009). In particular, it has been shown that under suitable conditions on the smoothness of the time-varying filter, the estimator is asymptotically normal (Theorem 3.2, Example 4.1 of Dahlhaus, 2009). We use this estimator in the initialization step of the procedure.

\subsubsection{Adaptive estimation of the time-dependent spectrum}

In order to data-adaptively smooth the pre-periodogram, we consider a sequence of weighted averages

$$
\hat{f}_{T}^{(k)}(u, \lambda)=\frac{1}{N_{u, \lambda}^{(k, T)}} \sum_{s, j} W_{u, \lambda}^{(k, T)}(s, j) J_{T}\left(\frac{s}{T}, \lambda_{j}\right) \quad k=1, \ldots, k_{\max }
$$

where $J_{T}$ is the pre-periodogram given in (2.9) and $N_{u, \lambda}^{(k, T)}=\sum_{s, j} W_{u, \lambda}^{(k, T)}(s, j)$ is the sum of weights. These weight sequences will thus determine the shape of the kernels of all points in the time-frequency plane. In each iteration $k$, we first construct in the penalty step an auxiliary kernel estimator

$$
\tilde{f}_{T}^{(k)}(u, \lambda)=\frac{1}{\tilde{N}_{u, \lambda}^{(k, T)}} \sum_{s, j} \tilde{W}_{u, \lambda}^{(k, T)}(s, j) J_{T}\left(\frac{s}{T}, \lambda_{j}\right),
$$


where the adaptive kernel weights $\tilde{W}_{u, \lambda}^{(k, T)}$ depend not only on the distance between $(u, \lambda)$ and $\left(\frac{s}{T}, \lambda_{j}\right)$ but also on the difference between $\hat{f}_{T}^{(k-1)}(u, \lambda)$ and $\hat{f}_{T}^{(k-1)}\left(\frac{s}{T}, \lambda_{j}\right)$, the estimates constructed in the previous iteration. More precisely, for fixed $(u, \lambda)$, the weights $\tilde{W}_{u, \lambda}^{(k, T)}$ are given by

$$
\tilde{W}_{u, \lambda}^{(k, T)}(s, j)=K_{\mathrm{f}}\left(\frac{\lambda-\lambda_{j}}{\tilde{b}_{\mathrm{f}, T, u, \lambda}^{(k)}}\right) K_{\mathrm{t}}\left(\frac{u-s / T}{\tilde{b}_{\mathrm{t}, T, u, \lambda}^{(k)}}\right) K_{\mathrm{p}}\left(P^{(k, T)}\left[(u, \lambda),\left(\frac{s}{T}, \lambda_{j}\right)\right]\right)
$$

and $\tilde{N}_{u, \lambda}^{(k, T)}=\sum_{s, j} \tilde{W}_{u, \lambda}^{(k, T)}(s, j)$. The bandwidths $\tilde{b}_{\mathrm{t}, \mathrm{T}, u, \lambda}^{(k)}$ and $\tilde{b}_{\mathrm{f}, \mathrm{T}, u, \lambda}^{(k)}$ of the localization kernels increase in $k$ to allow a larger degree of smoothing in each iteration. These bandwidths can differ over the plane and are based on the previous effective bandwidth. That is,

$$
\tilde{b}_{\mathrm{t}, \mathrm{T}, u, \lambda}^{(k)}=b_{\mathrm{T}, u, \lambda}^{(k-1)} \gamma_{t}, \text { and } \tilde{b}_{\mathrm{f}, \mathrm{T}, u, \lambda}^{(k)}=2 \pi b_{\mathrm{T}, u, \lambda}^{(k-1)} \gamma_{f},
$$

where $\gamma_{t}$ and $\gamma_{t}$ are the growth rates in time and frequency direction, respectively. Without loss of generality, the effective bandwidth is measured by $\left(b_{\mathrm{T}, u, \lambda}^{(k)}\right)^{2}=$ $N_{u, \lambda}^{(k, T)} / T^{2}$. To prevent over-smoothing, a penalty statistic $P^{(k, T)}\left[(u, \lambda),\left(\frac{s}{T}, \lambda_{j}\right)\right]$ is used to compare the two local estimates $\hat{f}_{T}^{(k-1)}(u, \lambda)$ and $\hat{f}_{T}^{(k-1)}\left(\frac{s}{T}, \lambda_{j}\right)$ from the previous iteration, and any deviation in value is penalized by means of the penalty kernel $K_{\mathrm{p}}$. Thus the resulting kernel assigns less weight to pre-periodogram variates that do not belong - based on the estimates from the previous step - to the local area of homogeneity about the point $(u, \lambda)$. We note that due to this construction the adaptive kernel in principle can be of any shape. If no penalization occurs, we obtain the usual kernel estimator in (2.11). Throughout the rest of the chapter, the latter estimator will be referred to as the nonadaptive estimator.

The estimator at step $k, \hat{f}_{T}^{(k)}(u, \lambda)$, is obtained by shifting the auxiliary estimator $\tilde{f}_{T}^{(k)}(u, \lambda)$ towards the estimator $\hat{f}_{T}^{(k-1)}(u, \lambda)$ from the previous step $k-1$. This is done in the memory step. More precisely, the kernel weights $W_{u, \lambda}^{(k, T)}(s, j)$ for the estimator $\hat{f}_{T}^{(k)}(u, \lambda)$ in $(2.12)$ are given by the linear combination

$$
W_{u, \lambda}^{(k, T)}(s, j)=\left(1-\theta_{u, \lambda}^{(k)}\right) \tilde{W}_{u, \lambda}^{(k, T)}(s, j)+\theta_{u, \lambda}^{(k)} W_{u, \lambda}^{(k-1, T)}(s, j) .
$$

Similarly, the effective sum of weights is given recursively by $N_{u, \lambda}^{(k, T)}=\left(1-\theta_{u, \lambda}^{(k)}\right) \tilde{N}_{u, \lambda}^{(k, T)}+$ $\theta_{u, \lambda}^{(k)} N_{u, \lambda}^{(k-1, T)}$. Here, $\theta_{u, \lambda}^{(k)}$ takes values in [0,1] based on the difference between the auxiliary estimator $\tilde{f}_{T}^{(k)}(u, \lambda)$ and the previous estimator $\hat{f}_{T}^{(k-1)}(u, \lambda)$. The more the two estimators differ the more weight is given to the previous estimate. This ensures a smooth transition of the estimators between iteration steps and prevents the algorithm from gradually accumulating estimation errors. This memory-step is essential in the current setting of time-varying spectral densities that exhibit smooth changes in both time and frequency direction. Although (Polzehl and Spokoiny, 2006) also consider a memory step to allow for smoother changes, in our case it also plays a crucial role in reducing the effect of cross-interference terms. We discuss this in more detail in section 2.3.5. 


\subsubsection{Penalty step}

The penalty statistic underlying the penalty kernel is an essential part of the procedure. At each iteration, it determines in which direction and to what degree smoothing occurs. We motivate the construction of our statistic as follows. In case the true spectrum is flat, the statistic must be such that no actual penalization occurs and by the end of the procedure our adaptive estimates ought to coincide with nonadaptive estimates obtained with global bandwidths $b_{\mathrm{t}}=1$ and $b_{\mathrm{f}}=2 \pi$. In other words, free extension of the smoothing bandwidths over the entire time-frequency plane should imply a completely homogeneous true spectrum. The scenario we are interested in is therefore whether two spectral estimates at different points can be considered to belong to the same 'homogeneous' region. In order to construct the test statistic we note that for the nonadaptive estimator given in (2.11), we have under certain regularity conditions (section 2.3.8), the following result:

$$
T b_{\mathrm{t}, T} b_{\mathrm{f}, T}\left(\hat{f}_{T}\left(u_{1}, \lambda_{1}\right)-f\left(u_{1}, \lambda_{1}\right)\right)^{2} \stackrel{\mathcal{D}}{\rightarrow} 2 \pi \kappa_{\mathrm{t}} \kappa_{\mathrm{f}} f^{2}\left(u_{1}, \lambda_{1}\right) \chi_{1}^{2},
$$

where $\kappa_{\mathrm{t}}=\int K_{\mathrm{t}}^{2}(x) d x$ and $\kappa_{\mathrm{f}}=\int K_{\mathrm{f}}^{2}(x) d x$. This implies

$$
T b_{\mathrm{t}, T} b_{\mathrm{f}, T}\left(\hat{f}_{T}\left(u_{1}, \lambda_{1}\right)-\hat{f}_{T}\left(u_{2}, \lambda_{2}\right)\right)^{2} \stackrel{\mathcal{D}}{\rightarrow} 4 \pi \kappa_{\mathrm{t}} \kappa_{\mathrm{f}} f^{2}\left(u_{1}, \lambda_{1}\right) \chi_{1}^{2},
$$

if homogeneity is satisfied. The latter result will be used as a general guideline to determine the degree of smoothing. More specifically, when constructing the new weights for the estimator at point $\left(u_{1}, \lambda_{1}\right)$, the result in $(2.18)$ can be applied to 'test' if $\hat{f}_{T}\left(u_{2}, \lambda_{2}\right)$ belongs to the confidence interval of $\hat{f}_{T}\left(u_{1}, \lambda_{1}\right)$.

\section{Penalty statistic}

The result in (2.18) motivates to consider a penalty statistic given by

$$
P^{(k+1, T)}\left(\left(u_{1}, \lambda_{1}\right),\left(u_{2}, \lambda_{2}\right)\right)=\frac{\tilde{N}_{u_{1}, \lambda_{1}}^{(k, T)}}{2 \pi \kappa_{\mathrm{f}} \kappa_{\mathrm{t}} T}\left(\frac{\hat{f}_{T}^{(k)}\left(u_{1}, \lambda_{1}\right)-\hat{f}_{T}^{(k)}\left(u_{2}, \lambda_{2}\right)}{\bar{f}_{T}^{(k)}\left(u_{1}, \lambda_{1}\right)}\right)^{2} .
$$

Here, the factor $\tilde{N}_{u_{1}, \lambda_{1}}^{(k, T)}$ measures the bandwidth of the adaptive kernel, e.g., for a nonadaptive kernel we would have $2 \pi \tilde{N}_{u_{1}, \lambda_{1}}^{(k, T)} \approx b_{\mathrm{t}, T} b_{\mathrm{f}, T} T^{2}$. If the value of the penalty statistic is close to zero and $\hat{f}_{T}^{(k)}\left(u_{2}, \lambda_{2}\right)$ can be considered to belong to the same region as $\hat{f}_{T}^{(k)}\left(u_{1}, \lambda_{1}\right)$, then the penalty kernel provides full weight to the corresponding pre-periodogram variate $J_{T}\left(u_{2}, \lambda_{2}\right)$ in the construction of the new estimator $\tilde{f}_{T}^{(k+1)}\left(u_{1}, \lambda_{1}\right)$. As in the nonadaptive case, it will then only be down weighted based on its absolute distance to the current point of interest by means of the kernels $K_{\mathrm{f}, T}$ and $K_{\mathrm{t}, T}$. From (2.19), it is easily observed that as the effective sum of weights increases and hence variability decreases, penalization becomes more severe. Moreover, it can be seen that this statistic is not symmetric but is based on the values of $N^{(k, T)}$ and $\bar{f}_{T}^{(k)}$ evaluated only around the point of interest, in this case $\left(u_{1}, \lambda_{1}\right)$. The reason is that for the type of processes we are interested in, i.e., 
smooth spectra with possible breaks, homogeneity is in general only a meaningful concept at a local level. In practice, we therefore need to take into account that energy levels differ over the plane. An important part of our algorithm is therefore the construction of the term $\bar{f}_{T}^{(k)}\left(u_{1}, \lambda_{1}\right)$, which replaces the denominator and takes into account the local energy- and noise level.

\section{Construction of the denominator}

More specifically, the unknown spectral density in the denominator has been replaced by

$$
\bar{f}_{T}^{(k)}\left(u_{1}, \lambda_{1}\right)=\overline{\hat{f}_{T}^{(k)}}\left(u_{1}, \lambda_{1}\right)+\hat{\sigma}_{T}^{(k)}\left(u_{1}, \lambda_{1}\right) .
$$

Here,

$$
\overline{\hat{f}_{T}^{(k)}}\left(u_{1}, \lambda_{1}\right)=\frac{1}{N^{*}} \sum_{s, j}\left|\hat{f}_{T}^{(k)}\left(\frac{s}{T}, \lambda_{j}\right)\right| 1_{\left\{\left|s / T-u_{1}\right| \leqslant b_{\mathrm{t}, T}^{(k) *},\left|\lambda_{j}-\lambda_{1}\right| \leqslant b_{\mathrm{f}, T}^{(k) *}\right\}}
$$

with $N^{*}=\sum_{s, j} 1_{\left\{\left|s / T-u_{1}\right| \leqslant b_{\mathrm{t}, T}^{(k) *},\left|\lambda_{j}-\lambda_{1}\right| \leqslant b_{\mathrm{f}, T}^{(k) *}\right\}}$ defines the local mean level over the neighborhood of $\left(u_{1}, \lambda_{1}\right)$ defined by the bandwidths $b_{\mathrm{f}, T}^{(k) *}$ and $b_{\mathrm{t}, T}^{(k) *}$. Correspondingly,

$$
\hat{\sigma}_{T}^{(k)}\left(u_{1}, \lambda_{1}\right)^{2}=\frac{1}{N^{*}} \sum_{s, j}\left[\hat{f}_{T}^{(k)}\left(\frac{s}{T}, \lambda_{j}\right)-\overline{\hat{f}_{T}^{(k)}}\left(u_{1}, \lambda_{1}\right)\right]^{2} 1_{\left\{\left|s / T-u_{1}\right| \leqslant b_{\mathrm{t}, T}^{(k) *},\left|\lambda_{j}-\lambda_{1}\right| \leqslant b_{\mathrm{f}, T}^{(k) *}\right\}},
$$

denotes the local variation about the local mean level over the same neighborhood. The motivation behind expression (2.20) is directly related to the iterative nature of the procedure and the underlying raw estimator. In particular, the method must not be too sensitive in areas with low signal to noise ratios and when the plane exhibits fast changes or high levels of curvature. The term (2.20) controls for this and we shall discuss the role of its respective two components in turn.

(i) Local average $\overline{\hat{f}_{T}^{(k)}}\left(u_{1}, \lambda_{1}\right)$ : Since $(2.20)$ is replacing the true spectrum at point $\left(u_{1}, \lambda_{1}\right)$ it needs to be representable for the local level of energy. Instead of replacing it with the point estimator, we use a local average as given in (2.21). The main reason is that we require it to be a stable representative value for the level of energy not only at the point of interest but also in a direct neighborhood around the point. If two adjacent points $\hat{f}_{T}^{(k)}\left(u_{i}, \lambda_{i}\right)$, $i=1,2$, have very different energy levels and the denominator of the corresponding statistics $P^{(k+1, T)}\left(\left(u_{1}, \lambda_{1}\right),\left(u_{2}, \lambda_{2}\right)\right)$ and $P^{(k+1, T)}\left(\left(u_{2}, \lambda_{2}\right),\left(u_{1}, \lambda_{1}\right)\right)$ are very different, the values of these statistics will also differ substantially. Consequently, the corresponding weights in their respective updated estimates for step $k+1$ will also be quite different. Especially in areas with high curvature, this could result in wrongful separation as the procedure continues. To make the method more robust against too early separation, we thus average locally in such a way that (2.21) is a reasonably smooth function over the plane.

(ii) Local noise measure $\hat{\sigma}_{T}^{(k)}\left(u_{1}, \lambda_{1}\right)$ : By definition, it gives a measure of local variation about the local mean level of energy in the neighborhood. A high value indicates that the local average (2.21) is off with respect to the actual 
level of energy directly at the point of interest $\left(u_{1}, \lambda_{1}\right)$. Accordingly, such a high value will result in less severe penalization by (2.20). This is of essence at the beginning of the procedure when the estimated plane is suffering from more noise as well as when local homogeneity starts to get violated and effective bandwidths and consequently noise levels start to differ. It then facilitates the possibility that optimal effective bandwidths might be reached earlier in certain areas while in others smoothing could still yield considerable improvement.

Remark 2.3.1. Note moreover that the local average in (2.21) is taken over the absolute set of estimates. This ensures that regions in which negative estimates occur, caused by the cross-interference terms underlying the pre-periodogram, are not over penalized. This feature, together with the memory step and the adjusted pre-periodogram (2.9), allows our method to reduce the effect of cross-interference terms to a minimum.

In the neighborhood of a break in the spectrum, the denominator term (2.20) will also take larger values. Nevertheless, breaks are still detected by our method if they locally dominate the smooth changes. In that case, the difference term in the nominator in (2.19) will approximately stay constant for two points on either side of the break whereas the total weight $N_{u, \lambda}^{(k, T)}$ of the kernels increases with $k$ and thus leads to effective penalization of all values on the opposite side of the break. Consequently, all weight of the kernel given by $\tilde{W}_{u, \lambda}^{(k+1, T)}$ is distributed to those values that are on the same side of the break as $(u, \lambda)$. In contrast, if the spectral density is smooth in the neighborhood of the point $(u, \lambda)$, the difference in the nominator in (2.19) changes only gradually as points further away from $(u, \lambda)$ are considered. This leads to penalization kernels that decay smoothly and change smoothly between points. A large value of $\hat{\sigma}_{T}^{(k)}\left(u_{1}, \lambda_{1}\right)$ will then delay strong penalization and thus facilitates further propagation. The effect of $\hat{\sigma}_{T}^{(k)}\left(u_{1}, \lambda_{1}\right)$ on the penalization statistic is negligible in case the plane exhibits local homogeneity. In order to reduce its importance if this is violated, the bandwidths $b_{\mathrm{f}, T}^{(k) *}$ and $b_{\mathrm{t}, T}^{(k) *}$ are reduced iteratively at a rate that is proportional to the rate at which the effective bandwidth grow. In areas where local homogeneity is not satisfied, this ensures that in later stages of the algorithm $\bar{f}_{T}^{(k)}\left(u_{1}, \lambda_{1}\right)$ is converging to the actual level of energy at the point of interest $\left(u_{1}, \lambda_{1}\right)$.

Remark 2.3.2 (An alternative statistic). To avoid the problem of standardization, we additionally investigated a log version of the statistic given by

$$
P_{I I}^{(k+1, T)}\left(\left(u_{1}, \lambda_{1}\right),\left(\lambda_{2}, \lambda_{2}\right)\right)=\frac{N_{u_{1}, \lambda_{1}}^{(k, T)}}{2 \pi \kappa_{\mathrm{t}} \kappa_{\mathrm{f}} T}\left(\log \left(\hat{f}_{T}^{(k)}\left(u_{1}, \lambda_{1}\right)\right)-\log \left(\hat{f}_{T}^{(k)}\left(u_{2}, \lambda_{2}\right)\right)\right)^{2} .
$$

Since the spectral density in the asymptotic variance cancels when considering logratios, the statistic does not require normalization by the unknown spectral density and thus is symmetric. The advantage is that less parameters need to be specified and the estimation method is simpler. However, the highly volatile behavior of the pre-periodogram and the possibility of negative estimates makes this version more problematic. For the log statistic it is essential to bound the initial estimates sufficiently far away from zero, while our original can deal with possible negative 
values. Moreover, it does not provide the same data-driven adaptation to local characteristics that allows for both smoothness and breaks. More details on this version are available upon request.

\subsubsection{The memory step}

Another important aspect of our procedure is the memory step which takes care of two problems. Firstly, it controls for the 'bad' behavior of the pre-periodogram. Secondly, it controls for accumulation of errors in areas where the spectrum is only gradually changing. In such regions, the adaptive bandwidths can increase even though the assumption of homogeneity has been violated. The larger neighborhood usually first leads to a drop in the estimation error but after some iteration the violation will result in an increasingly larger error which is carried over to next iterations. Imposing memory ensures that estimation precision reached in preceding iterations does not get lost and is used to bias, to some extent, the smoothing kernel to stability.

During the step, the new estimator $\hat{f}_{T}^{(k)}(u, \lambda)$ is constructed by shifting the auxiliary estimator $\tilde{f}_{T}^{(k)}(u, \lambda)$ towards the estimator $\hat{f}_{T}^{(k-1)}(u, \lambda)$ from the previous iteration. The size of the shift depends on the difference of the auxiliary and the previous estimator. More precisely, the shift is determined by $\theta_{u, \lambda}^{(k)}$, which is given by

$$
1-\theta_{u, \lambda}^{(k)}=(1-\eta) K_{\text {mem }}\left(P_{\text {mem }}^{(k, T)}(u, \lambda)\right)
$$

and defines the actual amount of memory. $\theta_{u, \lambda}^{(k)}$ takes values in $[\eta, 1]$, where the constant $\eta \in[0,1]$ specifies the minimal amount of memory imposed on the procedure. Furthermore, $K_{\mathrm{mem}}(x)$ is a kernel that is monotonically decreasing for $x \geqslant 0$ with $K(0)=1$. Similar to the penalty statistic, we consider

$$
P_{\text {mem }}^{(k, T)}(u, \lambda)=\frac{N_{u, \lambda}^{(k, T)}}{2 \pi \kappa_{\mathrm{t}} \kappa_{\mathrm{f}} T}\left(\frac{\tilde{f}_{T}^{(k)}(u, \lambda)-\hat{f}_{T}^{(k-1)}(u, \lambda)}{\bar{f}_{T}^{(k)}(u, \lambda)}\right)^{2} .
$$

The term $\bar{f}_{T}^{(k)}(u, \lambda)$ in the denominator is defined as in the penalty step but may be based on a smaller neighborhood (specified by bandwidths $b_{\mathrm{t}, T}^{(k) * *}$ and $b_{\mathrm{f}, T}^{(k) * *}$ ) about the point $\left(u_{1}, \lambda_{1}\right)$. The new effective estimate $\hat{f}_{T}^{(k)}\left(u_{1}, \lambda_{1}\right)$ is then obtained by a linear combination of the kernel weights for the auxiliary estimator $\tilde{f}_{T}^{(k)}(u, \lambda)$ and the previous estimator $\hat{f}_{T}^{(k-1)}(u, \lambda)$, i.e.,

$$
W_{u, \lambda}^{(k, T)}(s, j)=\left(1-\theta_{u, \lambda}^{(k)}\right) \tilde{W}_{u, \lambda}^{(k, T)}(s, j)+\theta_{u, \lambda}^{(k)} W_{u, \lambda}^{(k-1, T)}(s, j) .
$$

Similarly, the total sum of effective kernel weights is given by $N_{u, \lambda}^{(k, T)}=(1-$ $\left.\theta_{u, \lambda}^{(k)}\right) \tilde{N}_{u, \lambda}^{(k, T)}+\theta_{u, \lambda}^{(k)} N_{u, \lambda}^{(k-1, T)}$. The new estimator in terms of the auxiliary estimator and previous estimator can thus be given by

$$
\hat{f}_{T}^{(k)}\left(u_{1}, \lambda_{1}\right)=\frac{\left(1-\theta_{u, \lambda}^{(k)}\right) \tilde{f}_{T}^{(k)}(u, \lambda)+\theta_{u, \lambda}^{(k)} \hat{f}_{T}^{(k-1)}(u, \lambda)}{\left(1-\theta_{u, \lambda}^{(k)}\right) \tilde{N}_{u, \lambda}^{(k, T)}+\theta_{u, \lambda}^{(k)} N_{u, \lambda}^{(k-1, T)}} .
$$


If the value of the penalty is big, $\theta^{(k)}$ will be large and hence more weight is given to the previous update allowing to retain estimation accuracy obtained in the preceding steps. The higher the value of $\eta$ the more smooth the transition between old and new updates. At the same time, in case the changes are not gradual but rather strong discontinuities are present, higher values of $\eta$ will slow down how fast a certain level of precision is reached. As we require a balance between smooth transitions and the possibility of breaks it should not be set too high. We recommend $\eta \leqslant 0.25$. Subsequent updates of the same point as used in the memory penalty statistic are not independent and are expected to get more correlated as iterations continue. We take this into account by penalizing more severely as $k$ increases by using the adaptive (bigger) sum of weights belonging to the new adaptive estimate as given by $N_{u, \lambda}^{(k, T)}$ and a much lower cut-off value. Note moreover that as long as the growth rates are set high enough, the level of correlation can be managed. We will explain this in more detail in section 2.4.

Remark 2.3.3 (Controlling for cross-interference terms). Besides from reducing accumulation of estimation errors, the memory step is crucial in controlling for possible negative initial estimates caused by cross-interference terms present in the pre-periodogram. In order to retrieve localized features, it is best to keep the initial bandwidths as small as possible. This means the initial estimates are affected by cross-interference terms and hence can be negative. The memory step enables to remove these iteratively. This is done by imposing full memory in case the new update is more negative than its previous value. Together with the construction of the denominator of the penalty step, this leads them to be gradually pushed towards a positive value and prevents the rest of the plane to be affected.

Remark 2.3.4. We note that in our case the memory step is an essential part of the procedure both to control for this 'bad' behavior of the underlying pre-periodogram and the local slowly changing dynamics in the spectrum. This is in contrast to Becker and Mathé (2013) who consider piecewise constant functions in the time domain and conclude that such a relaxation step is redundant in their case.

\subsubsection{The procedure}

We now turn to the procedure in which we distinguish between the fully adaptive and the effective parameters/estimators by providing them with a tilde $\tilde{r}$ and a hat $\hat{\imath}$ respectively. The complete procedure is then as follows:

1. Select parameters: the bandwidth parameters $b_{\mathrm{t}, T}^{(0)}, b_{\mathrm{f}, T}^{(0)}, \gamma_{\mathrm{t}}, \gamma_{\mathrm{f}}, k_{\max }$ (start, growth rates, maximum number of iterations). Moreover, we will need to set the parameters $b_{\mathrm{t}, T}^{(k) *}, b_{\mathrm{f}, T}^{(k) *}$ and $b_{\mathrm{t}, T}^{(k) * *}, b_{\mathrm{f}, T}^{(k) * *}$ for the penalty- and memory statistic, respectively. For ease of exposition, we will postpone specification of the parameters to section 2.4 .

Then for all $u=\frac{t}{T}, \lambda=\lambda_{i}$ in the time-frequency plane: 
2. Initialisation: Construct the initial set of weights

$$
W_{u, \lambda}^{(\mathrm{in}, T)}(s, j)=\frac{1}{b_{\mathrm{f}, T}^{(0)}} K_{\mathrm{f}}\left(\frac{\lambda-\lambda_{j}}{b_{\mathrm{f}, T}^{(0)}}\right) \frac{1}{b_{\mathrm{t}, T}^{(0)}} K_{\mathrm{t}}\left(\frac{u-\frac{s}{T}}{b_{\mathrm{t}, T}^{(0)}}\right)
$$

and construct the initial set of estimates, denoted by $\hat{f}_{T}^{\mathrm{in}}(u, \lambda)$. Using these, construct the first set of fully adaptive weights $\left\{(s, j): \tilde{W}_{u, \lambda}^{(0, T)}(s, j)\right\}$ and estimates $\tilde{f}_{T}^{(0)}(u, \lambda)$ and accordingly the effective estimates $\hat{f}_{T}^{(0)}(u, \lambda)$ and sum of weights $N_{u, \lambda}^{0, T}$ by following step 3 and 4 below. Set $k=1$.

3. Penalty step: Compute the value of the penalty statistic based on the effective estimates $\hat{f}_{T}^{(k-1)}$ of the previous step. That is, compute

$$
P^{(k, T)}\left[(u, \lambda),\left(\frac{s}{T}, \lambda_{j}\right)\right]
$$

using (2.19) and accordingly the corresponding adaptive weights

$$
\tilde{W}_{u, \lambda}^{(k, T)}(s, j)=K_{\mathrm{f}}\left(\frac{\lambda-\lambda_{j}}{\tilde{b}_{\mathrm{f}, \mathrm{T}, u, \lambda}^{(k)}}\right) K_{\mathrm{t}}\left(\frac{u-\frac{s}{T}}{\tilde{b}_{\mathrm{t}, \mathrm{T}, u, \lambda}^{(k)}}\right) K_{\mathrm{p}}\left(P^{(k, T)}\left[(u, \lambda),\left(\frac{s}{T}, \lambda_{j}\right)\right]\right),
$$

in order to construct the fully adaptive estimator $\tilde{f}_{T}^{(k)}(u, \lambda)$.

4. Memory step: Compare $\tilde{f}_{T}^{(k)}(u, \lambda)$ with $\hat{f}_{T}^{(k-1)}(u, \lambda)$, the previous effective estimate based on the memory penalty statistic $P_{\operatorname{mem}}^{(k)}(u, \lambda)$ as defined in $(2.25)$ and compute the effective set of weights

$$
W_{u, \lambda}^{(k, T)}(s, j)=\left(1-\theta_{u, \lambda}^{(k)}\right) \tilde{W}_{u, \lambda}^{(k, T)}(s, j)+\theta_{u, \lambda}^{(k)} W_{u, \lambda}^{(k-1, T)}(s, j)
$$

and effective estimate

$$
\hat{f}_{T}^{(k)}\left(u_{1}, \lambda_{1}\right)=\frac{\left(1-\theta_{u, \lambda}^{(k)}\right) \tilde{f}_{T}^{(k)}(u, \lambda)+\theta_{u, \lambda}^{(k)} \hat{f}_{T}^{(k-1)}(u, \lambda)}{\left(1-\theta_{u, \lambda}^{(k)}\right) \tilde{N}_{u, \lambda}^{(k, T)}+\theta_{u, \lambda}^{(k)} N_{u, \lambda}^{(k-1, T)}} .
$$

Set $k=k+1$.

5. Stopping rule: Repeat steps 3 and 4 until $k=k_{\max }$.

Remark 2.3.5. In order to obtain the first set of fully adaptive estimates $\{(u, \lambda)$ : $\left.\tilde{f}_{T}^{(0)}(u, \lambda)\right\}$, the penalty statistic is based on the initial set of nonadaptive estimates $\left\{(u, \lambda): \hat{f}_{T}^{\mathrm{in}}(u, \lambda)\right\}$. The first set of effective estimates $\left\{(u, \lambda): \hat{f}_{T}^{0}(u, \lambda)\right\}$ is then obtained by applying the memory statistic to $\tilde{f}_{T}^{(0)}(u, \lambda)$ and $\hat{f}_{T}^{\text {in }}(u, \lambda)$ for all $u, \lambda$.

\subsubsection{Computational complexity}

The algorithm has been implemented in $\mathrm{R}$ using the Rcpp package in order to make use of the compiler language $\mathrm{C}++$. In particular, certain parts of the algorithm are implemented using the OpenMP interface for carrying out parallel computations in a shared memory environment. The reason for this is the complexity of the algorithm. 
For a time series of length $T$, the time-frequency plane will consist of $T^{2}$ data points. Using the FFT algorithm, computing the pre-periodogram variates is an operation of order $O(T \ln (T))$. The computational cost lies however in the quantity of weights to construct. In step $k$, the maximum number of weights to compute for the new estimator of step $k+1$, will approximately be between $\frac{3}{8 \pi} b_{\mathrm{t}, \mathrm{T}}^{(0)} \gamma_{t}^{(k+1)} b_{\mathrm{f}, \mathrm{T}}^{(0)} \gamma_{f}^{(k+1)} T^{2}$ and $\frac{1}{2 \pi} b_{\mathrm{t}, T}^{(k+1)} \gamma_{t}{ }^{(k+1)} b_{\mathrm{f}, \mathrm{T}}^{(0)} \gamma_{f}^{(k+1)} T^{2}$, depending on whether we are in the middle of the plane or at the boundaries. Determining a weight value, i.e., constructing the kernel values, and additionally the sums of weights is an operation of order $O(1)$. The maximum complexity of the construction for the new estimator at one particular point is therefore of order $O\left(b_{\mathrm{t}, T}^{(k+1)} b_{\mathrm{f}, T}^{(k+1)} T^{2}\right)$. It follows that the total complexity of the algorithm is $O\left(b_{\mathrm{t}, T}^{\left(k_{\max }\right)} b_{\mathrm{f}, T}^{\left(k_{\max }\right)} T^{4}\right)$. We remark however that for most points in the plane, the final effective bandwidths $\tilde{b}_{\mathrm{t}, \mathrm{T}, u, \lambda}^{\left(k_{\max }\right)}$ and $\tilde{b}_{\mathrm{f}, \mathrm{T}, u, \lambda}^{\left(k_{\max }\right)}$, which are computed using (2.15), will be much lower than $b_{\mathrm{t}, \mathrm{T}}^{(0)} \gamma_{f}^{\left(k_{\max }\right)}$ and $b_{\mathrm{f}, \mathrm{T}}^{(0)} \gamma_{f}^{\left(k_{\max }\right)}$.

\subsubsection{Asymptotic considerations}

The objective of this section is to provide some intuition on the distributional properties of the adaptive estimator in (2.13). We do this in the setting of empirical spectral processes (e.g. Dahlhaus and Polonik, 2009; Dahlhaus, 2009), on which we first provide some background. Generally, the empirical spectral process for arbitrary index functions $\phi$ is defined by

$$
E_{T}(\phi)=\sqrt{T}\left(F_{T}(\phi)-F(\phi)\right)
$$

where

$$
F(\phi)=\int_{0}^{1} \int_{-\pi}^{\pi} \phi(u, \lambda) f(u, \lambda) d u d \lambda
$$

is the generalized spectral measure and

$$
F_{T}(\phi)=\frac{1}{T} \sum_{t=1}^{T} \int_{-\pi}^{\pi} \phi\left(\frac{t}{T}, \lambda\right) J_{T}\left(\frac{t}{T}, \lambda\right) d \lambda
$$

denotes the corresponding empirical spectral measure. For particular classes of index functions independent of $T$, a functional central limit theorem has been proved (Dahlhaus and Polonik, 2009, Theorem 2.11). Additionally, for index functions depending on $T$ a central limit theorem has been derived (Dahlhaus, 2009, Theorem 3.2). Both results are shown under the following conditions on the corresponding stochastic process. Here, let

$$
V(g)=\sup _{0 \leqslant y_{0} \leqslant \ldots \leqslant y_{m} \leqslant 1, m \in \mathbb{N}} \sum_{k=1}^{m}\left|g\left(y_{k}\right)-g\left(y_{k-1}\right)\right|,
$$

denote the total variation of a function $g$ on $[0,1]$ and let $l(j)=\max \left\{1,|j| \log ^{1+\varsigma}|j|\right\}$ for some $\varsigma>0$. 
Assumption 2.3.6. $\left\{X_{t, T}\right\}$ is locally stationary according to Definition 2.2.1 such that the coefficients $a_{t, T}(j), j \in \mathbb{N}$ of the linear filter defined by the transfer function $A_{t, T}(\lambda)$ satisfy

$$
\sup _{t, T}\left|a_{t, T}(j)\right| \leqslant \frac{C}{l(j)},
$$

for some constant $C$ not depending on $T$. Moreover, the coefficients $a(u, j), j \in \mathbb{N}$, of the linear filter defined by the transfer function $A(u, \lambda)$ satisfy

$$
\begin{gathered}
\sup _{u \in[0,1]}|a(u, j)| \leqslant \frac{C}{l(j)}, \\
\sup _{j \in \mathbb{N}} \sum_{t=1}^{T}\left|a_{t, T}(j)-a\left(\frac{t}{T}, j\right)\right| \leqslant C, \\
V(a(\cdot, j)) \leqslant \frac{C}{l(j)} .
\end{gathered}
$$

Many localized statistics for nonstationary time series can be written in terms of the empirical spectral measure. In particular, we obtain the nonadaptive timevarying spectral estimator in (2.10) by considering index functions

$$
\phi_{u, \lambda}^{(T)}(v, \mu)=\frac{1}{b_{\mathrm{t}, T} b_{\mathrm{f}, T}} K_{\mathrm{t}}\left(\frac{u-v}{b_{\mathrm{t}, T}}\right) K_{\mathrm{f}}\left(\frac{\lambda-\mu}{b_{\mathrm{f}, T}}\right) .
$$

Since the index functions depend on $T$, asymptotic normality of the estimator $F_{T}\left(\phi_{u, \lambda}^{(T)}\right)$ and its discretized version (2.11) follows from Theorem 3.2 and Example 4.1 of Dahlhaus (2009) under the following additional conditions.

\section{Assumption 2.3.7.}

(i) The time-varying spectral density $f(u, \lambda)$ is twice differentiable in $u$ and $\lambda$ with uniformly bounded derivatives.

(ii) The bandwidths satisfy $b_{\mathrm{t}, T}, b_{\mathrm{f}, T} \rightarrow 0$ and $b_{\mathrm{t}, T} b_{\mathrm{f}, T} T \gg \log (T)^{2}$ as $T \rightarrow \infty$,

(iii) The kernels $K_{\mathrm{t}}$ and $K_{\mathrm{f}}$ are of bounded variation with compact support. Moreover, $\int x K_{\mathrm{t}}(x) d x=0$ and $\int K_{\mathrm{t}}(x) d x=1$ and analogously for $K_{\mathrm{f}}$.

In particular, we find that

$$
b_{\mathrm{t}, T} b_{\mathrm{f}, T} \operatorname{var}\left(E_{T}\left(\phi_{u, \lambda}^{(T)}\right)\right) \rightarrow 2 \pi f(u, \lambda) \kappa_{\mathrm{t}} \kappa_{\mathrm{f}} .
$$

Furthermore, estimators at different points in the time-frequency plane are asymptotically independent.

For the adaptive estimator in (2.13) similar asymptotic results cannot be derived easily since the final smoothing kernel is iteratively defined and depends on the spectral estimators in previous steps through penalization and the memory step. In the following, we therefore provide at least heuristic arguments that under homogeneity of the spectral density penalization has a negligible effect and hence the estimator remains consistent and asymptotically normal.

More precisely assume that $f(u, \lambda)=f$ for all $u$ and $\lambda$ and define for fixed $u \in[0,1]$ and $\lambda \in[-\pi, \pi]$ the functions

$$
\psi_{\alpha, \beta}=\phi_{u+\alpha b_{\mathrm{t}, T}, \lambda+\beta b_{\mathrm{f}, T}}^{(T)}
$$


where $\phi_{u, \lambda}^{(T)}$ is defined as above. Then the family of index functions $\mathscr{F}_{0}=\left\{\psi_{\alpha, \beta} \mid \alpha, \beta \in\right.$ $[-1,1]\}$ satisfies the conditions of Theorem 2.11 of Dahlhaus and Polonik (2009). Hence the penalty statistic $P^{(k, T)}\left[(u, \lambda),\left(u+\alpha b_{\mathrm{t}, T}, \mu+\beta b_{\mathrm{f}, T}\right)\right]$ asymptotically has that same distribution as

$$
\frac{b_{\mathrm{t}, T} b_{\mathrm{f}, T}}{2 \pi \kappa_{\mathrm{f}} \kappa_{\mathrm{t}} f^{2}}\left(E\left(\psi_{0,0}\right)-E\left(\psi_{\alpha, \beta}\right)\right)^{2},
$$

where $E(\psi)$ is a Gaussian process with mean zero and covariances

$$
\begin{array}{r}
b_{\mathrm{t}, T} b_{\mathrm{f}, T} \operatorname{cov}\left(E\left(\psi_{\alpha, \beta}\right), E\left(\psi_{\gamma, \delta}\right)\right)=2 \pi \\
f^{2} \int_{-1 / 2}^{1 / 2} \int_{-\pi}^{\pi} K_{\mathrm{t}}(\alpha-u) K_{\mathrm{t}}(\gamma-u) K_{\mathrm{f}}(\beta-\lambda) \\
\times\left[K_{\mathrm{f}}(\delta-\lambda)+K_{\mathrm{f}}(\delta+\lambda)\right] d u d \lambda+O\left(b_{\mathrm{f}, T}\right) .
\end{array}
$$

The expression shows that under the assumption of homogeneity of the time-varying spectrum over the local neighborhood about the point $(u, \lambda)$ the distribution of the penalty statistic does not depend on the bandwidth or the sample size but through a term of order $O\left(b_{\mathrm{f}, T}\right)$. Moreover, the strong positive correlation of the Gaussian process $E(\psi)$ leads to at most weak penalization towards the borders of the local neighborhood yielding a total smoothing kernel that differs only slightly from the nonadaptive smoothing kernel. Finally, since $E\left(\psi_{0,0}\right)$ and $E\left(\psi_{\alpha, \beta}\right)$ are positively correlated, the variance of their difference can be bounded by $2 \operatorname{var}\left(E\left(\psi_{0,0}\right)\right)$ uniformly for all $\alpha, \beta \in[-1,1]$, which justifies the use of (2.18) for determining the cut-off point of the penalty kernel.

We note that the same covariance structure can be derived from Theorem 3.2 of Dahlhaus (2009) by considering the index functions $\phi_{u+\alpha b_{\mathrm{t}, T}, \lambda+\beta b_{\mathrm{f}, T}}^{(T)}$ directly, that is, taking their dependence on $T$ into account in the asymptotics. However, the result is weaker insofar it does not yield convergence over the whole local neighborhood defined by $\alpha, \beta \in[-1,1]$ simultaneously. Although the above arguments based on fixed index functions indicate that this result could be strengthened, a derivation of a functional central limit theorem in this setting is beyond the scope of this chapter.

Summarizing we find that under the assumption of homogeneity penalization does only modify the shape of the smoothing kernel even if applied iteratively multiple times but will keep the rates approximately the same. In contrast, in case of a non-constant spectral density, the penalty statistic depends quadratically on the difference in levels which leads to more severe penalization as bandwidths in time and frequency direction increase. Accordingly, the resulting smoothing kernel will have in general a smaller support corresponding to a smaller bandwidth than the one actually imposed. Nevertheless, in the setting of locally stationary processes this effect will disappear asymptotically since the level of local homogeneity increases as long as the bandwidths used in the iteration satisfy the conditions in Assumption 2.3.7. In other words, the adaptive estimator remains consistent with rate $\sqrt{T b_{\mathrm{t}, T}^{\left(k_{\max }\right)} b_{\mathrm{f}, T}^{\left(k_{\max }\right)}}$ since its adaptiveness only shows in finite samples. This is even true when the dynamics of the process exhibits structural breaks and thus should be described by a piecewise locally stationary process. In that case, penalization will be strong in the local neighborhood of a break leading to asymmetric smoothing kernels 
that seem to be cut off. Again, since the local neighborhoods (in the rescaled timefrequency plane) are shrinking for increasing sample size, the effect will disappear but for the points along the breaks where the time-varying spectral density is not well-defined. Examples of such processes with structural breaks will be discussed in Section 5, where we illustrate the final sample behavior of the adaptive estimator by simulations.

\section{Bias}

Under assumption 2.3.7, it is easily derived that the bias under local homogeneity can approximately be given by

$$
\frac{1}{2} b_{\mathrm{t}, T}^{(k)^{2}} \int x^{2} K_{\mathrm{t}}(x) d x \frac{\partial^{2}}{\partial u^{2}} f(u, \lambda)+\frac{1}{2} b_{\mathrm{f}, T}^{(k)^{2}} \int x^{2} K_{\mathrm{f}}(x) d x \frac{\partial^{2}}{\partial \lambda^{2}} f(u, \lambda)+o(1),
$$

and thus will be negligible for bandwidths satisfying

$$
\sqrt{T b_{\mathrm{t}, T}^{(k)} b_{\mathrm{f}, T}^{(k)}}\left(b_{\mathrm{t}, T}^{(k)^{2}}+b_{\mathrm{f}, T}^{(k)^{2}}\right) \rightarrow 0 \text { as } T \rightarrow \infty .
$$

In the asymptotics, this needs to be balanced with the other assumption on the bandwidths underlying the central limit theorem (Assumption 2.3.7(ii)) and constitutes the usual bias-variance trade-off. When $T$ is fixed, the bias present in $\hat{f}_{T}^{(k)}\left(u_{1}, \lambda_{1}\right)$ and $\hat{f}_{T}^{(k)}\left(u_{2}, \lambda_{2}\right)$ cancels out in the penalty statistic under local homogeneity. Moreover, in case the bias starts to differ either due to differences in effective bandwidths or a difference in curvature, then this be detected by the penalty statistic through $\tilde{\sigma}_{T}^{(k)}\left(u_{1}, \lambda_{1}\right)$ or $\tilde{\sigma}_{T}^{(k)}\left(u_{2}, \lambda_{2}\right)$, translating in a higher value of (2.20) and in turn reducing the risk of wrongful rejection due to difference in bias. Because of this property of the penalty statistic to adjust to bias, taking into account 2.3.7(ii) will be of more importance when specifying the initial bandwidths $b_{\mathrm{t}, T}^{(0)}$ and $b_{\mathrm{f}, T}^{(0)}$, than the bias criteria.

\subsection{Specification of parameters}

In this section, we discuss some guidelines on how to set the parameters of the procedure. As long as these guidelines are followed our method is robust under different processes and additional fine-tuning is not necessary.

\subsubsection{Bandwidth parameters}

\section{Initial bandwidths}

It is clear that the smaller the initial bandwidth the more details can be retrieved. This is however under the constraint that the initial set of nonadaptive estimates are not dominated by the variability and, in particular cross-interference terms, of the underlying pre-periodogram as they need to be stable enough to be used in the penalty statistic. The guideline is therefore to take the smallest bandwidths for 
which the smoothed pre-periodogram obeys the condition underlying the central limit theorem 2.3.7(iv); $b_{\mathrm{t}, T}^{(0)} b_{\mathrm{f}, T}^{(0)} T \gg \log (T)^{2}$. For example, when $T=512$, we set $b_{\mathrm{t}, T}^{(0)} \approx 0.12, \frac{1}{2 \pi} b_{\mathrm{f}, T}^{(0)} \approx 0.12$, which can be reduced for larger values of $T$.

For the additional bandwidth parameters required for the penalty statistics (2.19) and $(2.25)$, respectively, we set $b_{\mathrm{t}, T}^{(k) *}=b_{\mathrm{t}, T}^{(k) * *}=b_{\mathrm{t}, T}^{(0)}, b_{\mathrm{f}, T}^{(k) *}=b_{\mathrm{f}, T}^{(k) * *}=b_{\mathrm{f}, T}^{(0)}$. As explained in section 2.3.4, these are reduced over iterations based on the rate at which the effective bandwidth of the $q$-th percentile is growing with respect to the starting bandwidths. We recommend $10 \leqslant q \leqslant 20$, to make sure this decrease goes gradually and at the same time is not affected by possible negative estimates.

\section{Growth rates}

The growth rates determine how much 'localization' takes place. If bandwidths increase at a high rate, the effect of the penalty statistic becomes more pronounced and the transition between local homogeneous regions less smooth. If set too high, the procedure might overlook gradual change or interesting characteristics. For example, when a region with similar energy level is included in the new larger neighborhood we could miss some feature in between. This is to some extent corrected for by the two kernels that penalize distance but could occur when two small peaks of similar energy are close to each other or close to a large peak, a common problem in spectral density estimation.

A lower bound on the growth rates is necessary to control violation of the independence assumption underlying the asymptotic distribution of the penalty statistic. To give some intuition, consider the algorithm at step $k$. We have estimates $\hat{f}_{T}^{(k-1)}\left(u_{i}, \lambda_{j}\right)$ for all $\left(u_{i}, \lambda_{j}\right) \in U \times \Pi$ and the bandwidths are set to $\tilde{b}_{\mathrm{t}, \mathrm{T}, u, \lambda}^{(k)}=\gamma_{\mathrm{t}} b_{\mathrm{T}, u, \lambda}^{(k-1)}$ and $\tilde{b}_{\mathrm{f}, \mathrm{T}, u, \lambda}^{(k)}=\gamma_{\mathrm{f}} b_{\mathrm{T}, u, \lambda}^{(k-1)}$. To compute the estimate $\hat{f}_{T}^{(k)}\left(u_{i}, \lambda_{j}\right)$, a new set of weights $\left\{\tilde{W}_{u_{i}, \lambda_{j}}^{(k, T)}(s, l)\right\}$ is constructed by comparing the estimate $\hat{f}_{T}^{(k-1)}\left(u_{i}, \lambda_{j}\right)$ with all other estimates $\hat{f}_{T}^{(k-1)}\left(u_{s}, \lambda_{l}\right)$ that fall into the new search neighborhood as given by the larger bandwidths. If the growth rates only allow a small increase of this neighborhood compared to the previous step, underlying weight sets and hence pre-periodogram variates will strongly overlap with one another. These estimates are therefore expected to be highly correlated with the current point of interest $\hat{f}_{T}^{(k-1)}\left(u_{i}, \lambda_{j}\right)$. This increases the risk that $P^{(k, T)}$ will redistribute weights within approximately the same area as in the previous step leading to falsely iterative exclusion of certain data points. It is thus of importance that the growth rates are high enough to make sure that a large part of weights is based on points that did not overlap in the previous step to ensure independence can be considered reasonable. Results show that rates $\gamma_{\mathrm{t}}$ and $\gamma_{\mathrm{f}}$ that satisfy $1.2 \leqslant \gamma_{\mathrm{t}} \gamma_{\mathrm{f}} \leqslant 1.5$ provide a good balance. 


\section{Maximal number of iterations}

The maximal iteration $k_{\max }$ is determined by two conditions. Firstly, the effective sum of weights needs to increase sufficiently compared to the previous effective sum of weights. This for the same reason as discussed above, i.e. to prevent the penalty statistic from reconsidering approximately the same areas as in the previous iterations. To measure this, denote

$$
\gamma^{(k)}\left(u_{i}, \lambda_{l}\right)=N_{\left(u_{i}, \lambda_{l}\right)}^{(k, T)} / N_{\left(u_{i}, \lambda_{l}\right)}^{(k-1, T)}
$$

The condition we impose is that the average over the time-frequency plane of the effective growth rate given by

$$
\bar{\gamma}^{(k)}=\frac{1}{T^{2}} \sum_{i, l} \gamma^{(k)}\left(u_{i}, \lambda_{l}\right)
$$

satisfies $\bar{\gamma}^{(k)}>\left(\gamma_{\mathrm{t}} \gamma_{\mathrm{f}}\right)^{0.25}$. Once this is no longer satisfied the algorithm stops. Additionally, the effect of bias (Remark 2.3.8) in case of local stationarity needs to be taken into account. As a proxy to measure when it starts to play a role, we use

$$
\min _{i, l} b_{\mathrm{T}, u_{i}, \lambda_{l}}^{(k)} \approx T^{-1 / 6}
$$

If the algorithm reaches such bandwidths, smoothing is stopped when the dispersion between the highest and lowest quartile of $\gamma^{(k)}$ over the time-frequency plane is larger than $10 \%$ of possible change, i.e.,

$$
\mathcal{Q}_{\gamma^{k}}(0.75)-\mathcal{Q}_{\gamma^{k}}(0.25)>0.1 \gamma_{\mathrm{t}} \gamma_{\mathrm{f}}
$$

Together these two conditions allow the method to automatically take into account the possibility for spectra with very steep and localized features and on the other hand to continue smoothing in case of spectra with large stationary or flat regions. The condition that the algorithm is keeping track of is therefore

$$
\begin{aligned}
k_{\max }=\underset{k}{\operatorname{argmin}} & \left(\left\{\bar{\gamma}^{(k)}>\left(\gamma_{\mathrm{t}} \gamma_{\mathrm{f}}\right)^{0.25}\right\}\right. \\
& \left.\cup\left\{\mathcal{Q}_{\gamma^{k}}(0.75)-\mathcal{Q}_{\gamma^{k}}(0.25)>0.1 \gamma_{\mathrm{t}} \gamma_{\mathrm{f}} \mid \min _{i, l} b_{\mathrm{T}, u_{i}, \lambda_{l}}^{(k)} \approx T^{-1 / 6}\right\}\right)
\end{aligned}
$$

Remark 2.4.1 (Growth rates in case of negative estimates). As explained in Remark 2.3.3, the memory step imposes full memory in case the auxiliary estimate is more negative than the previous effective estimate. For these point estimates, we require a lower growth rate $\rho$ for the new search area to make sure the cross-interference effect is smoothed out locally and not by 'accident'. We advise $1.01 \leqslant \rho \leqslant 1.03$ depending on the length of data. 


\subsubsection{Kernels}

The penalty kernel should be a concave function that tapers off softly. This because, even though the algorithm makes sure that the separation property is satisfied (weights that were zero remain zero), it will not necessarily stop redistributing the weights within a region considered 'homogeneous'. A very steep penalty kernel as used in Polzehl and Spokoiny (2006) pushes more and more weight in a smaller area. The kernels $K_{\mathrm{t}}(x)$ and $K_{\mathrm{f}}(x)$ would exaggerate this behavior. This is clearly not suitable in our application as it leads to piecewise constant areas in the estimated plane. Instead, we need 'smooth' rejection over iterations, i.e., as the effective bandwidths grow and variability is reduced, the effect of the penalty kernel becomes more important. To establish this, we use the concave function

$$
K_{\mathrm{p}}(x)=\left[1-\left(\frac{x}{c \rho^{k}}\right)^{2}\right] 1_{\left[0, c \rho^{k}\right]}(x),
$$

where the cut-off point is given by $c=2 \chi_{1,0.9}^{2}$ and is based on the asymptotic distribution of the penalty statistic under local homogeneity. The term $\rho^{k}$, controls that for unstable areas affected by cross-interference terms (Remark 2.4.1) the effect of the penalty statistic in the weight distribution does not gain importance. We note that at the beginning of the procedure, the assumed independence underlying the asymptotic distribution as given in (2.18) will not truly hold for adjacent points due to the overlap of raw estimates. In general, this is not a problem but rather provides initial support around the midpoint. For the memory kernel we take the linear kernel

$$
K_{\text {mem }}(x)=\left(1-\frac{x}{c \rho^{-k}}\right) 1_{\left[0, \rho^{-k} c_{m e m}\right]}(x),
$$

where the default choice for the cut-off point is $c_{m e m}=2 \chi_{1,0.75}^{2}$. We let the kernel decrease at a rate $\rho^{k}$ as we expect more correlation between the new and old estimator as iterations continue, especially in areas affected by the cross-interference terms.

For the distance kernels $K_{t}$ and $K_{f}$, we use quadratic kernels

$$
K_{\mathrm{t}}(x)=K_{\mathrm{f}}(x)=6\left(\frac{1}{4}-x^{2}\right) 1_{[-0.5,0.5]}(x),
$$

which have been shown to yield the smallest mean squared error (Dahlhaus, 1996b) in case the null of homogeneity is satisfied.

Remark 2.4.2. If it is suspected the length of the data is too short for the asymptotic distribution to be reasonable to use for the cut-off point, one can scale the statistics appropriately. The amount of scaling can be determined by the smallest constant such that the procedure returns close to nonadaptive estimates for a homogeneous spectrum with similar mean energy as the original process.

\subsection{Examples}

In this section we illustrate the proposed adaptive smoothing method by applying it to three examples that cover three types of situation: constant spectra with 
a structural break, smooth time-varying spectra, and time-varying spectra that also exhibit structural breaks. In all examples, we choose the same set of tuning parameters for the algorithm to demonstrate that the method is to some degree insensitive against choice of parameters.

In all examples, the sample length is $T=1024$ and the initial bandwidths for the localization kernels are $b_{\mathrm{t}, T}^{(0)}=b_{\mathrm{f}, T}^{(0)} / 2 \pi=0.1$. The growth rates are set to $\gamma_{\mathrm{t}}=\gamma_{\mathrm{f}}=1.2$ and $\rho=1.02$. The parameters for the penalty step and the memory step are $b_{\mathrm{t}, T}^{(k) *}=b_{\mathrm{t}, T}^{(0)}, b_{\mathrm{f}, T}^{(k) *}=b_{\mathrm{f}, T}^{(0)}, q=0.15, \eta=0.25$. The parameters for the kernels are the default choices as explained in the previous section. The corresponding figures ${ }^{1}$ are given in the appendix.

\subsubsection{Structural break white noise}

As a first example, we consider a break in a white noise series. That is, we consider the following model for the observations

$$
X_{t, T}=Z_{t} 1_{\left\{t \leqslant t_{0}\right\}}+\sqrt{10} Z_{t} 1_{\left\{t>t_{0}\right\}}, \quad Z_{t} \stackrel{\mathrm{iid}}{\sim} \mathcal{N}(0,1)
$$

with $t_{0}=576$. The corresponding time-varying spectral density is given by

$$
f(u, \lambda)=\frac{1}{2 \pi} 1_{\left\{u \leqslant t_{0} / T\right\}}+\frac{10}{2 \pi} 1_{\left\{u>t_{0} / T\right\}} .
$$

and is depicted in Figure 2.2(a). Figure $2.2(\mathrm{~b})$ shows the corresponding preperiodogram. Compared with the usual periodogram, it exhibits much more variation which completely blur the piecewise constant form of the density. The initial adaptive estimate for $k=0$ is given in Figure $2.2(\mathrm{c})$. Here, the change in level is already discernible but the estimate is still relatively non-smooth. Following (2.45), the algorithm stopped at $k_{\max }=11$ iterations and yields the estimated time-frequency plane in Figure $2.2(\mathrm{~d})$. It clearly shows two levels for the spectral density. Figures 2.3 (a)-(b) depict, respectively, the final penalty and adaptive kernel for the midpoint of the plane. Once the break is detected the penalty kernel forces the weights down to zero. This results in asymmetric smoothing kernel that is 'cut' off and thus succeeds in separating the areas on both sides of the break. To demonstrate the effect of the procedure, figure 2.2 (e) shows the estimated plane using the nonadaptive estimator as given in (2.11) with the same total search neighborhood, i.e., with bandwidths $\gamma_{\mathrm{t}}^{11}$ and $b_{\mathrm{t}, T}^{(0)}, \gamma_{\mathrm{f}}^{11} b_{\mathrm{f}, T}^{(0)}$. Not surprisingly, the presence of the break is completely smoothed out.

To benchmark how our method performs, we use a proxy for the optimal unknown global bandwidths for the nonadaptive estimator. To construct it, the mean squared error over the plane was computed for different bandwidths. We then took the one for which the error was lowest. To ease notation, we shall denote the corresponding estimator by $\hat{f}_{b_{\mathrm{opt}}}^{\mathrm{Na}}$, the nonadaptive estimator with same total search neighborhood as the final adaptive estimator by $\hat{f}^{\mathrm{Na}}$. The estimator from our procedure will then be denoted by $\hat{f}_{k_{\max }}^{\text {Ad }}$. Figure 2.4 (a) provides box plots of the squared

\footnotetext{
${ }^{1}$ All data is available upon request.
} 
error over the time-frequency plane of these estimator with respect to the true spectral variates, i.e., $\left(\hat{f}^{\cdot}(\cdot, \cdot)-f_{\text {true }}(\cdot, \cdot)\right)^{2}$ for $\hat{f}^{\cdot}=\hat{f}_{b_{\text {opt }}}^{\mathrm{Na}}, \hat{f}^{\cdot}=\hat{f}_{k_{\max }}^{\mathrm{Ad}}$ and $\hat{f}^{\cdot}=\hat{f}^{\mathrm{Na}}$. It is clear that our method does much better, except in the extrema. This is intuitive, since the nonadaptive estimator will smooth out extremes such as a break while our method will detect the break but might be slightly off in terms of location. To illustrate, figure 2.4 (b) provides the frequency average of $\hat{f}_{b_{\mathrm{opt}}}^{\mathrm{Na}}$ (red), $\hat{f}_{k_{\max }}^{\mathrm{Ad}}$ (blue) and the true spectrum $f_{\text {true }}$ (green). It is clear that we are really close to detecting the break but that we are a few data points off.

\subsubsection{Locally stationary series}

In the second example, we consider a time-varying moving average process of order 2 given by

$$
X_{t, T}=\cos \left(2 \pi \frac{t}{T}\right) Z_{t}-\left(\frac{t}{T}\right)^{2} Z_{t-1}, \quad Z_{t} \stackrel{\text { iid }}{\sim} \mathcal{N}(0,1)
$$

The time-varying spectral density of this process, given by

$$
f(u, \lambda)=\frac{1}{2 \pi}\left(\cos (2 \pi u)^{2}-2 u^{2} \cos (2 \pi u) \cos (\lambda)+u^{4}\right),
$$

is depicted in Figure 2.5 (a). The spectrum exhibits a peak in the middle of the time-frequency plane with smooth hill-sides in frequency direction and steeper ones in time direction. The pre-periodogram and the estimated plane after the adaptive iteration are given in 2.5 (b) and 2.5 (c). The iterations are stopped after $k_{\max }=7$ and the corresponding final estimated spectrum $\hat{f}_{k_{\max }}^{\mathrm{Ad}}$ is given in Figure $2.5(\mathrm{~d})$. The penalty and adaptive kernel for the midpoint $(u, \lambda)=(0.5,0)$ are provided in Figure 2.6. Barely any penalization is visible which is in line with the smoothness properties of the underlying process. However, we also observe (Fig. 2.5 (e)) that the nonadaptive estimated spectrum $\hat{f}^{\mathrm{Na}}$ with comparable bandwidths clearly out smooths the curvature. A comparison of the squared errors in Figure 2.7 (a) shows our adaptive procedure outperforms the optimal nonadaptive estimated spectrum. Although less convincing than in the previous case, this is still interesting as the original process is close to stationary. Over-smoothing by a global bandwidth will therefore not pose the same problem as it does for processes that exhibit a higher degree of nonstationarity. Figure 2.7 (b) gives the average over frequencies and demonstrates our method captures the curvature better than the nonadaptive estimates with optimal global bandwidths.

\subsubsection{Structural break in locally stationary series}

We now combine the above two processes. More precisely, we consider a white noise process that at $t_{0}=410$ turns into an moving average process with similar dynamics as in the previous example but shifted in time. Thus we have

$$
X_{t, T}=\sigma Z_{t} 1_{\left\{t \leqslant t_{0}\right\}}+\left[\cos \left(2 \pi\left(\frac{t}{T}-\frac{1}{5}\right)\right) Z_{t}-\left(\frac{t}{T}-\frac{1}{5}\right)^{2} Z_{t-1}\right] 1_{\left\{t>t_{0}\right\}}
$$


with $Z_{t} \stackrel{\text { iid }}{\sim} \mathcal{N}(0,1)$. The corresponding time-varying spectral density is given by

$$
f(u, \lambda)=\frac{\sigma^{2}}{2 \pi} 1_{\left\{u \leqslant t_{0} / T\right\}}+g\left(u-\frac{1}{5}, \lambda\right) 1_{\left\{u>t_{0} / T\right\}},
$$

where $g(u, \lambda)$ is as in (2.52). These type of spectra can for example occur when a signal is constant for a while and then receives a stimulus. This time-varying spectrum is interesting as the peak and the break are both close in distance as well as in level and hence are difficult to resolve. The estimation results are depicted in Figures 2.8 to 2.10 . The algorithm is also in this case stopped after $k=7$ iterations. For visual comparison, we additionally included (Fig. $2.8(\mathrm{~d})$ ) the nonadaptive estimated spectrum $\hat{f}^{\mathrm{Na}}$. It can be seen to suffer clearly from over-smoothing. Figure 2.9 (a)- (b) show strong penalization in time direction and close to none in frequency direction for the midpoint, which lies in the valley. This is in accordance with the different slopes in the two directions. Compared to the previous case, the better performance compared to the optimal global bandwidth proxy is much more pronounced (Fig. 2.8(a)). In particular, it can be seen in $2.10(\mathrm{~d})$, that the adaptive estimated plane again better captures the features of the true spectrum.

To see whether our conclusions depend on the variance level of the white noise before the structural break, we also consider the case where $\sigma^{2}=3$. The corresponding plots are in Figures 2.11 to 2.13. The penalty kernel of the midpoint now shows a quicker cut-off for the break point than in the lower variance case (Fig. 2.9 (a)-(b)). The adaptive kernel turns out as expected and gives full weight in frequency direction while it stops smoothing in time direction for areas outside the valley (Fig. 2.12 (a)-(b)). In terms of errors, also here our data-adaptive procedure shows a clear improvement compared to the optimal global bandwidth proxy. 


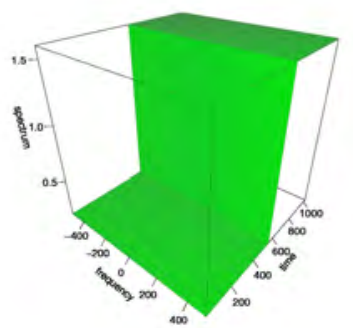

(a)

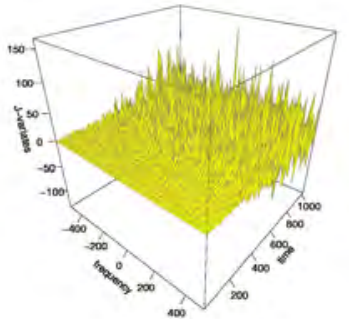

(b)

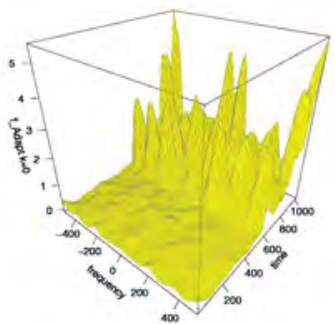

(c)

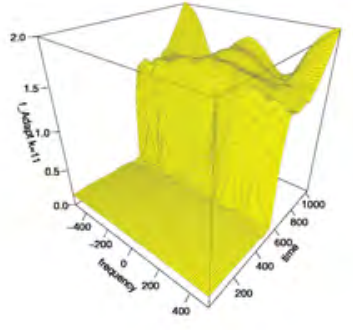

(d)

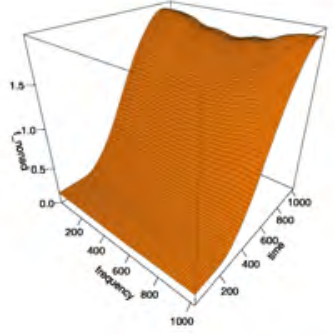

(e)

Figure 2.2: Example (2.5.1): true spectrum (a); pre-periodogram (b); adaptive estimated spectrum for $k=0(\mathrm{c})$ and for $k_{\max }=11(\mathrm{~d})$; nonadaptive estimated spectrum $\hat{f}^{\mathrm{Na}}(\mathrm{e})$.
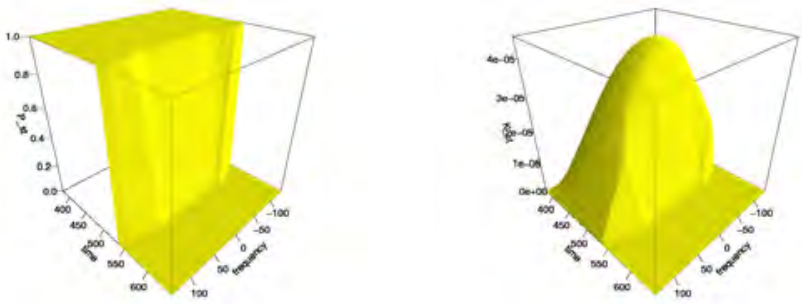

Figure 2.3: Penalty kernel (left) and adaptive kernel (right) $\hat{f}_{k_{\max }}^{\mathrm{Ad}}$ for $k_{\max }=11$ at $(u, \lambda)=(0.5,0)$.
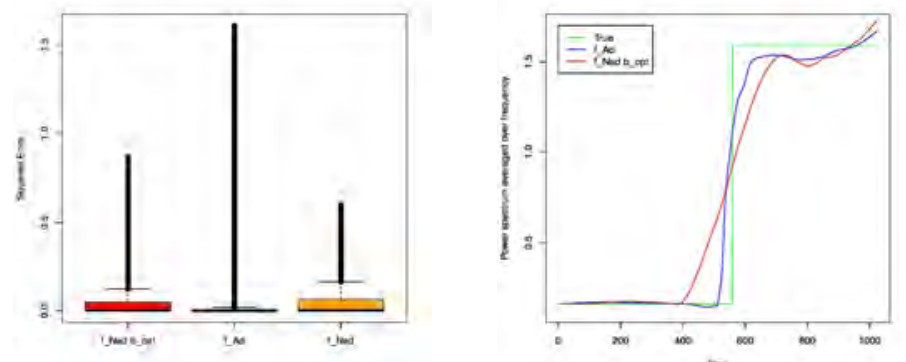

Figure 2.4: Squared error (left) and frequency average (right) of $\hat{f}_{b_{\mathrm{opt}}}^{\mathrm{Na}}, \hat{f}_{k_{\max }}^{\mathrm{Ad}}$ and $\hat{f}^{\mathrm{Na}}$, respectively. 


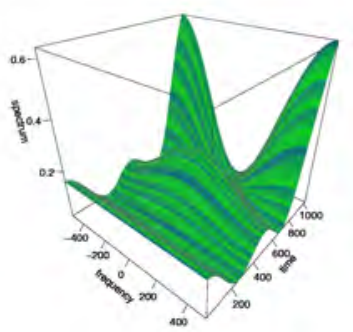

(a)

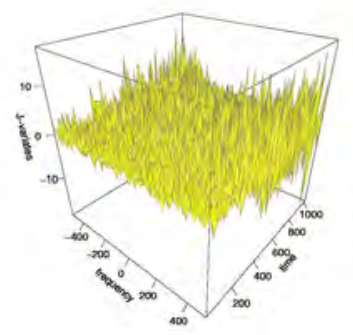

(b)

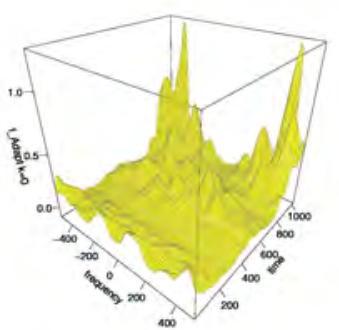

(c)

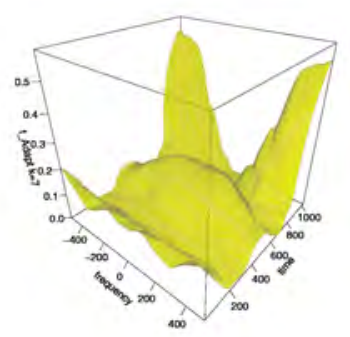

(d)

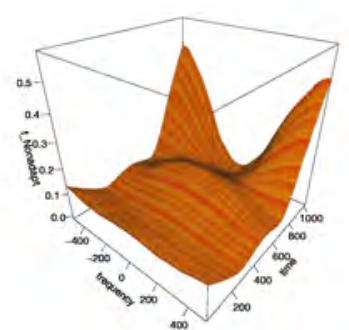

(e)

Figure 2.5: Example in (2.5.2): true spectrum (a); pre-periodogram (b); adaptive estimated spectrum for $k=0$ (c) and for $k_{\max }=7$ (d); nonadaptive estimated spectrum $\hat{f}^{\mathrm{Na}}$ (e).
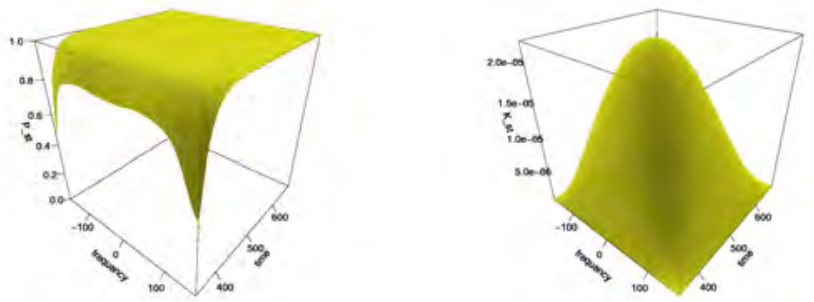

Figure 2.6: Penalty kernel (left) and adaptive kernel (right) $\hat{f}_{k_{\max }}^{\mathrm{Ad}}$ for $k_{\max }=7$ at $(u, \lambda)=$ $(0.5,0)$.
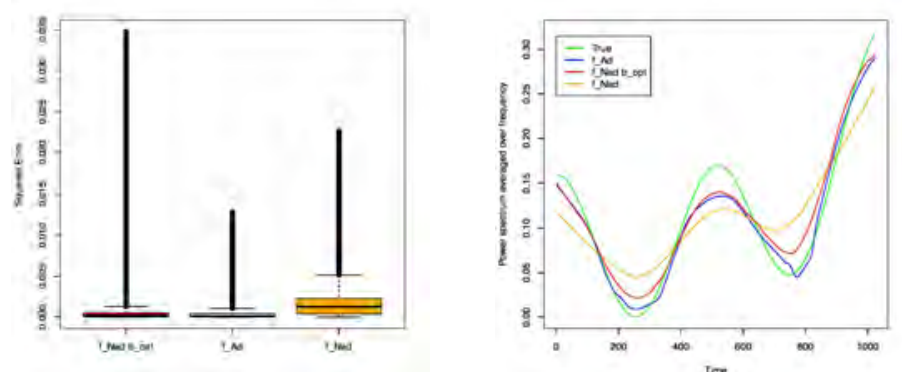

Figure 2.7: Squared error (left) and frequency average (right) of $\hat{f}_{b_{\mathrm{opt}}}^{\mathrm{Na}}, \hat{f}_{k_{\max }}^{\mathrm{Ad}}$ and $\hat{f}^{\mathrm{Na}}$, respectively. 


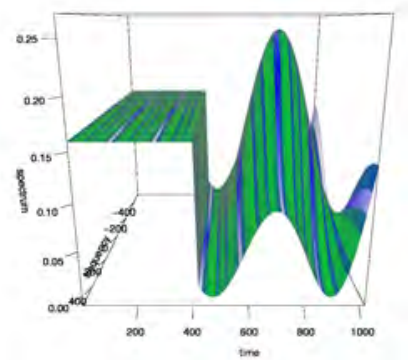

(a)

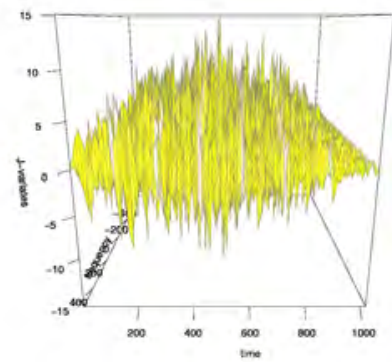

(b)

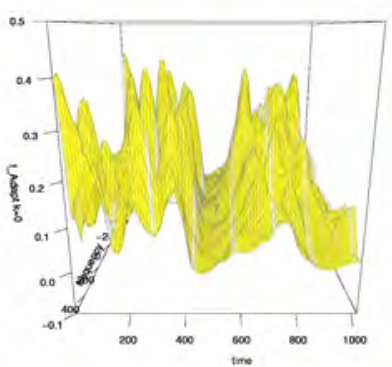

(c)

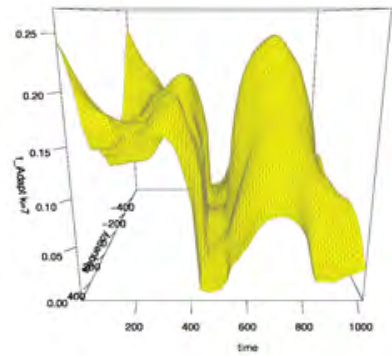

(d)

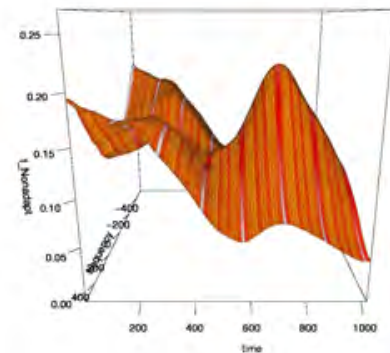

(e)

Figure 2.8: Example in (2.5.3) with $\sigma=1$ : true spectrum (a); pre-periodogram (b); adaptive estimated spectrum for $k=0$ (c) and for $k_{\max }=7$ (d); nonadaptive estimated spectrum $\hat{f}^{\mathrm{Na}}(\mathrm{e})$.
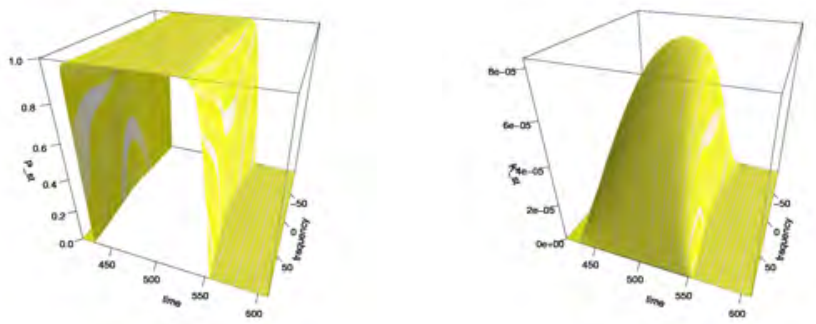

Figure 2.9: Penalty kernel (left) and adaptive kernel (right) $\hat{f}_{k_{\max }}^{\mathrm{Ad}}$ for $k_{\max }=7$ at $(u, \lambda)=$ $(0.5,0)$.
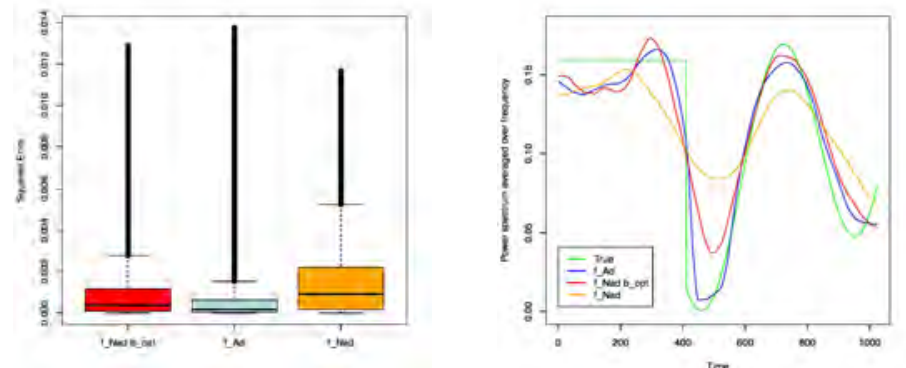

Figure 2.10: Squared error (left) and frequency average (right) of $\hat{f}_{b_{\text {opt }}}^{\mathrm{Na}}, \hat{f}_{k_{\max }}^{\mathrm{Ad}}$ and $\hat{f}^{\mathrm{Na}}$, respectively. 


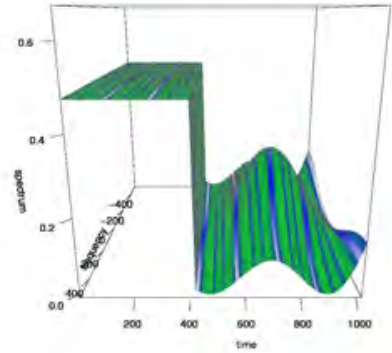

(a)

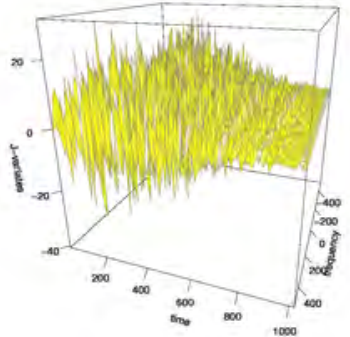

(b)

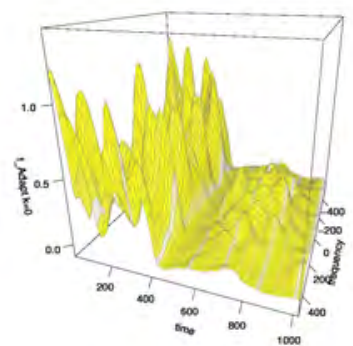

(c)

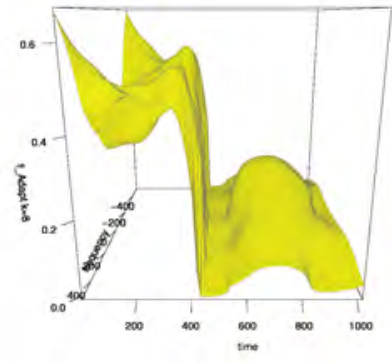

(d)

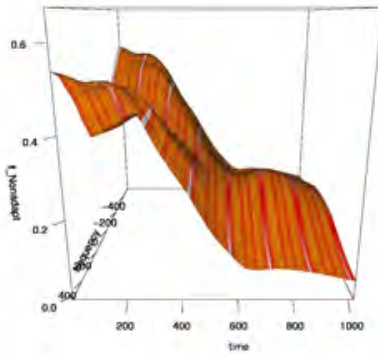

(e)

Figure 2.11: Example in (2.5.3) with $\sigma=3$ : true spectrum (a); pre-periodogram (b); adaptive estimated spectrum for $k=0$ (c) and for $k_{\max }=8$ (d); nonadaptive estimated spectrum $\hat{f}^{\mathrm{Na}}(\mathrm{e})$.
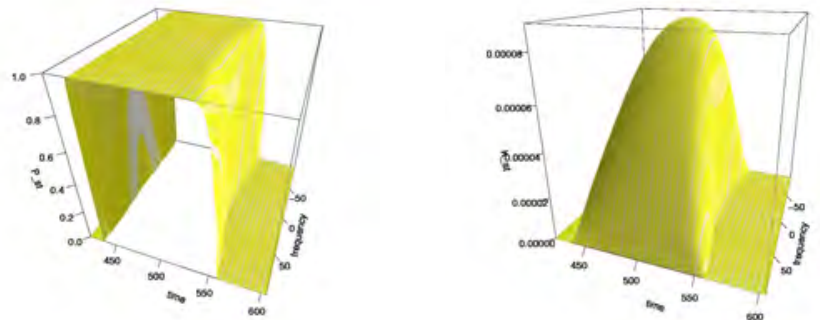

Figure 2.12: Penalty kernel (left) and adaptive kernel (right) $\hat{f}_{k_{\max }}^{\mathrm{Ad}}$ for $k_{\max }=8$ at $(u, \lambda)=(0.5,0)$.
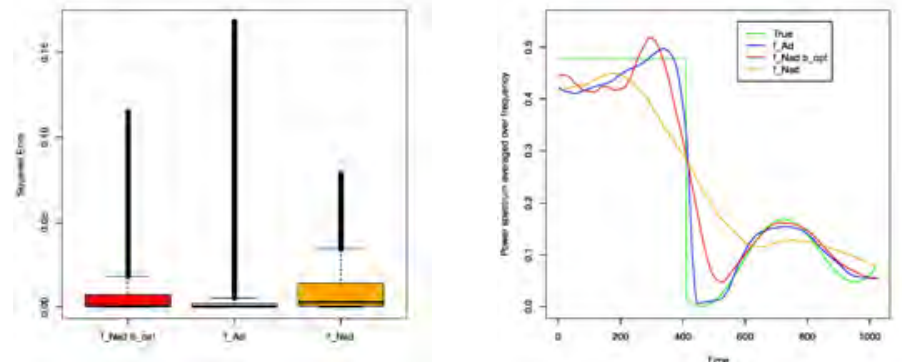

Figure 2.13: Squared error (left) and frequency average (right) of $\hat{f}_{b_{\text {opt }}}^{\mathrm{Na}}, \hat{f}_{k_{\max }}^{\mathrm{Ad}}$ and $\hat{f}^{\mathrm{Na}}$, respectively. 


\subsection{Application to Local Field Potentials}

As an application of our method, we consider Local Field Potential (LFP) recordings of the nucleas accumbens of a male macaque monkey during an associative learning experiment. The experiment was conducted at the Neurosurgery Department at the Massachusetts General Hospital. During each trial, the monkey was shown four pictures and a then had to select one of four doors. If the monkey made the correct association between picture and door he would receive a reward. In total, the learning experiment consisted of 675 trials. During the experiment, local field potentials were recorded of the nucleus accumbens and the hippocampus. In Fiecas and Ombao (2016), the nature of the dynamic interactions between these were investigated. There is clear evidence of nonstationary behavior within the trials, something that has also been observed in other types of brain data. It is therefore interesting to see if our method can capture this nonstationary behavior.

In Fig 2.14 we have the data of one of the trials. For our purposes, we consider the stretch of data $t \in[490: 1513]$ as this shows interesting behavior in time direction. A first inspection of the pre-periodogram (Fig 2.15(a)-(b)) indicates most of the neuronal activity is centered really close to frequency zero. In order to resolve the narrow peak(s), a small bandwidth is required in frequency direction. In particular, it is well-known from the stationary setting the width of the main lobe from the kernel function should be no larger and preferably half the size than the bandwidth of the narrowest peak in the spectrum. However, if it is set too conservative the erratic behavior of the pre-periodogram will be carried over to the estimates. Moreover, there is also clear evidence of nonstationary behavior in time direction (Fig 2.15 (c)). Given the length of data, any starting bandwidths for which Assumption 2.3.7 (ii) holds might out-smooth most of this behavior. In order to still capture the activity and deal with possible more volatile behavior, we set $b_{\mathrm{t}, T}^{(0)}=b_{\mathrm{f}, T}^{(0)}=0.05, \gamma_{t}=\gamma_{f}=1.1$ and $b_{T}^{(0) *}=b_{T}^{(0) * *}=b_{\mathrm{f}, T}^{(0)} / 2$. Additionally, $c=2 \chi_{1,0.99}^{2}$ and $c_{m e m}=2 \chi_{1,0.5}^{2}$ to take into account there will be more variation due to the small starting bandwidths. The rest of the parameters are the same as in section 2.5.

The estimated spectrum is given in Figure 2.16. Despite the fact that the stretch of data might not give enough resolution to resolve the exact peak location, it is clearly estimated away from zero. We observe some definite changes in both width and location of the peak over time. Figure 2.17 shows the estimated spectrum locally around the point $(u, \lambda)=(0.5,0.362)$. We see rough patches here and there due to the low starting bandwidths but overall good adjustment of the penaltyand final kernel to the local peak. Although some effect of the cross-interference terms are visible in the final estimate, the overall estimated time-frequency plane appears to capture local structures reasonably well. This is promising given that the assumption of the underlying penalty statistic are not completely satisfied. 

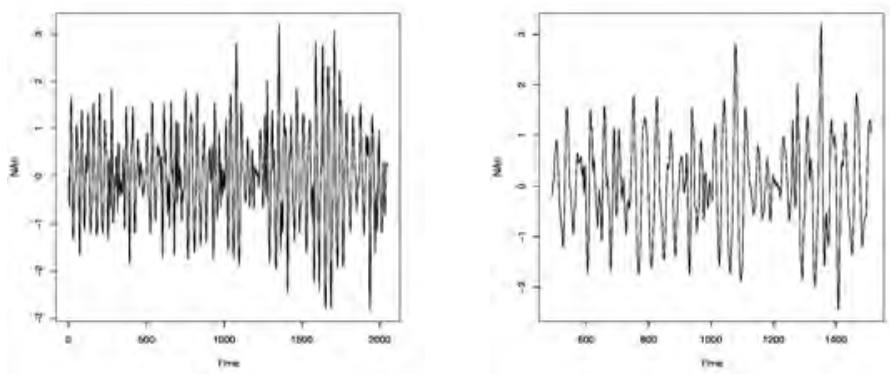

Figure 2.14: Time series Nac trial (left) and sub-stretch (right) over [490, 1513].

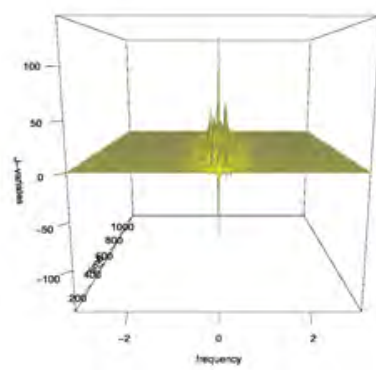

(a)

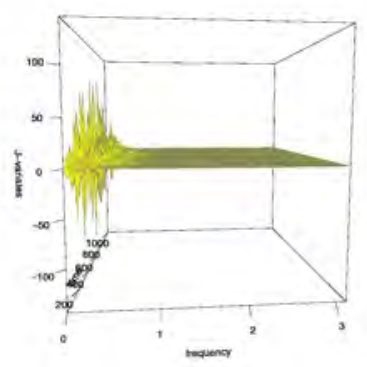

(b)

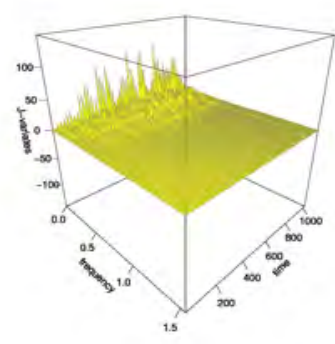

(c)

Figure 2.15: Pre-periodogram over $(-\pi, \pi]$ (a) and over $\lceil 0, \pi\rceil$ for different angles (b)-(c).
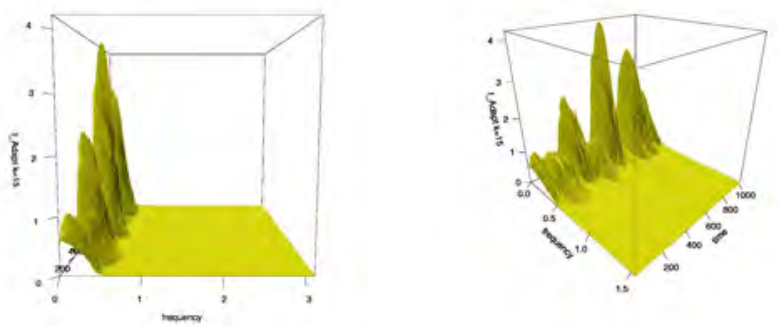

Figure 2.16: estimated spectrum $k_{\max }=15$ over $[0, \pi]$ (left) and over $[0, \pi / 2]$ for different angles (right).

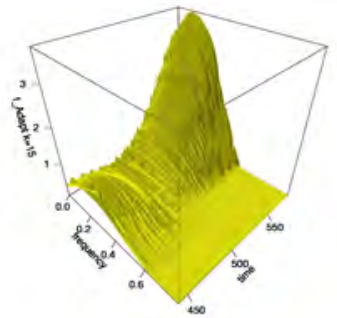

(a)

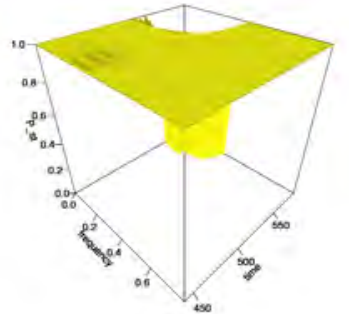

(b)

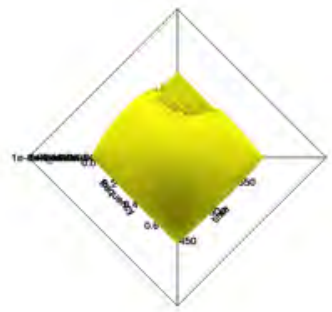

(c)

Figure 2.17: estimated spectrum $k_{\max }=15$ around point $(u, \lambda)=(0.5,0.362)$ (a) with penalty kernel (b) and adaptive kernel (c), respectively. 


\subsection{Conclusion}

In this chapter, we introduced an adaptive kernel smoothing method for timevarying spectral densities. The method conceptually is based on the propagationseparation algorithm by Polzehl and Spokoiny (2006) but differs from it on essential points.

Firstly, although the algorithm by Polzehl and Spokoiny allows some smoothness, it is particularly designed to model piecewise constant functions. It will separate regions for which homogeneity is violated in a very strict manner. As an implication, such a method will favor identifying a steep hill-side as a structural break rather than a smooth transition. In contrast, the spectral density of a time series - stationary or nonstationary - is rarely piecewise constant over frequencies. Moreover, capturing the valleys and hill-sides of peaks in the spectrum form a particular difficulty that are of a very different nature than what can be found in pure time domain analysis. Appropriately dealing with smoothly changing spectra requires that transitions from lower levels of energy to higher levels of energy are not being identified as a structural break. The method discussed in this chapter is tailored to take this into account. Our way of penalizing, by means of the penalty kernel, is such that we allow for a smoother transition between adjacent points so that too quick separation is prevented.

Secondly, the original propagation-separation method relies on a regularity condition that follows from the underlying assumption of an exponential family. Consequently, the sample should give more or less equal information for different local parameter values. This assumption will often be violated for processes of interest in this chapter since peaks and curvature are the rule rather than the exception. Consequently, the difference between optimal bandwidths over the plane could be substantial. Despite the essence of the problem being essentially the same, namely finding the optimal region adaptively for estimation, our underlying assumptions and framework are thus quite different.

Admittedly, our method has its limitations. In particular, even though the extension to multivariate spectra is direct, the running time can become quite long for the series that are of interest to us $(T>600)$. This is a drawback but can be solved by running the algorithm on the graphics processing unit. The other issue has to do with identification. In a way we run into the classical problem of trying to obtain both momentum and position and as noted in section 2.3.2, we cannot get them perfect at the same time. The balance that we need between optimal bandwidths and reliable updates requires that extremes should be over smoothed to some extent. However, the method allows for a high level of flexibility between final bandwidths for different points and the level of over-smoothing is much less than when a global bandwidth for all local variates is imposed. Empirical results show that in case of locally stationary processes with or without break, we obtain much lower errors. In particular, our method adjusts very well to the presence of a break in smooth changing spectra. The method is directly applicable to data and takes into account data features as presented to the researcher and does not rely too heavily on asymptotical results yet still obeys to its framework. Furthermore, 
it is robust under a variety of processes without fine-tuning of the parameters. It would be worth looking into the theoretical properties in more detail, possibly allowing optimizing parameter specification. Additionally, it is of general interest to investigate a method that optimally reduces the effect of cross-interference terms of the underlying pre-periodogram. This is left for future research. 



\section{Chapter 3}

\section{Locally stationary functional time series}

\subsection{Introduction}

In functional data analysis, the variables of interest take the form of smooth functions that vary randomly between repeated observations or measurements. Thus functional data are represented by random smooth functions $X(\tau), \tau \in D$, defined on a continuum $D$. Examples of functional data are concentration of fine dust as a function of day time, the growth curve of children as functions of age, or the intensity as a function of wavelength in spectroscopy. Because functional data analysis deals with inherently infinite-dimensional data objects, dimension reduction techniques such as functional principal component analysis (FPCA) have been a focal point in the literature. Fundamental for these methods is the existence of a Karhunen-Loève decomposition of the process (Karhunen, 1947; Loève, 1948). Some noteworthy early contributions are Kleffe (1973); Grenander (1981); Dauxois et al. (1982); Besse and Ramsay (1986). For an introductory overview of the main functional data concepts we refer to Ramsay and Silverman (2005) and Ferraty and Vieu (2006).

Most techniques to analyze functional data are developed under the assumption of independent and identically distributed functional observations and focus on capturing the first- and second-order structure of the process. A variety of functional data is however collected sequentially over time. In such cases, the data can be described by a functional time series $\left\{X_{t}(\tau): t \in \mathbb{Z}\right\}$. Since such data mostly show serial dependence, the assumption of i.i.d. repetitions is violated. Examples of functional time series in finance are bond yield curves, where each function is the yield of the bond as a function of time to maturity (e.g. Bowsher and Meeks, 2008; Hays et al., 2012) or the implied volatility surface of a European call option as a function of moneyness and time to maturity. In demography, mortality and fertility rates are given as a function of age (e.g. Erbas et al., 2007; Hyndman and Ullah, 2007; Hyndman and Booth, 2008), while in geophysical sciences, magnometers record the strength and direction of the magnetic field every five seconds. Due to the wide range of applications, functional time series and the development of techniques that 
allow to relax the i.i.d. assumption have received an increased interest in recent years.

The literature on functional time series has mainly centered around stationary linear models (Mas, 2000; Bosq, 2002; Dehling and Sharipov, 2005). In particular, the dissertation by Bosq (2000) investigates the theory of linear functional time series in Banach spaces with emphasis on the functional autoregressive model. Prediction methods have also attracted a reasonable amount of attention (Antoniadis et al., 2006; Bosq and Blanke, 2007; Aue et al., 2015). A general framework to investigate the effect of temporal dependence among functional observations on existing techniques has been provided by Hörmann and Kokoszka (2010), who introduce $L_{m}^{p}$-approximability as a moment-based notion of dependence.

Violation of the assumption of identically distributed observations has been examined in the setting of change-point detection (e.g. Berkes et al., 2009; Hörmann and Kokoszka, 2010; Aue et al., 2009; Horváth et al., 2010; Gabrys et al., 2010), in the context of functional regression by Yao et al. (2005); Cardot and Sarda (2006) and in the context of common principal component models by Benko et al. (2009).

Despite the growing literature on functional time series, the existing theory has so far been limited to strongly or weakly stationary processes. With the possibility to record, store and analyze functional time series of an increasing length, the common assumption of (weak) stationarity becomes more and more implausible. For instance, in meteorology the distribution of the daily records of temperature, precipitation and cloud cover for a region, viewed as three related functional surfaces, may change over time due to global climate changes. In the financial industry, implied volatility of an option as a function of moneyness changes over time. While heuristic approaches such as localized estimation are readily implemented and applied, a statistical theory for inference from nonstationary functional time series is yet to be developed.

The objective of the current chapter is to develop a framework for inference of nonstationary functional time series that allows the derivation of large sample approximations for estimators and test statistics. For this, we extend the concept of locally stationary processes (Dahlhaus, 1996a) to the functional time series setting. Our work draws from previous work of Panaretos and Tavakoli (2013b,a) who derived a Cramér representation for weakly stationary functional time series and discussed the frequency domain-based inference in this setting.

The chapter is structured as follows. In section 3.2, we introduce some basic notation and methodology for functional data and relate this in a heuristic manner to the concept of locally stationary time series and introduce the definition of a locally stationary functional time series. In section 3.3, the time-varying spectral density operator is defined and its properties are derived. In particular, we will show uniqueness. In section 3.4, we demonstrate that time-varying functional ARMA models have a causal solution and are functional locally stationary according to the definition as provided in section 3.2. Then in section 3.5, we derive the distributional properties of a local nonparametric estimator of the time-varying spectral density operator and deduce a central limit theorem. 


\subsection{Locally stationary functional time series}

Let $\left\{X_{t}\right\}_{t=1, \ldots, T}$ be a stochastic process taking values in the Hilbert space $H=$ $L^{2}([0,1], \lambda)$ of all real-valued functions that are square integrable with respect to the Lebesgue measure $\lambda$. While current theory for such processes is limited to the case where $X_{t}$ is either strictly or weakly stationary, we consider nonstationary processes with dynamics that vary slowly over time and thus can be considered as approximately stationary at a local level.

As an example, consider the functional autoregressive process $\left\{X_{t}\right\}$ given by

$$
X_{t}(\tau)=B_{t}\left(X_{t-1}\right)(\tau)+\varepsilon_{t}(\tau), \quad \tau \in[0,1]
$$

for $t=1, . ., T$, where the errors $\varepsilon_{t}$ are independent and identically distributed random elements in $H$ and $B_{t}$ for $t=1, \ldots, T$ are bounded operators on $H$. Assuming that the autoregressive operators $B_{t}$ change only slowly over time, we can still obtain estimates by treating the process as stationary over short time periods. However, since this stationary approximation deteriorates over longer time periods, standard asymptotics based on an increasing sample size $T$ do not provide suitable distributional approximations for the finite sample estimators. Instead we follow the approach by Dahlhaus $(1996 a, 1993)$ and define local stationary processes in a functional setting based on an infill asymptotics. The main idea of this approach is that for increasing $T$ the operator $B_{t}$ is still 'observed' on the same interval but on a finer grid, resulting in more and more observations in the time period over which the process can be considered as approximately stationary. Thus we consider a family of functional processes

$$
X_{t, T}(\tau)=B_{t / T}\left(X_{t-1, T}\right)(\tau)+\varepsilon_{t}(\tau), \quad \tau \in[0,1], \quad 1 \leqslant t \leqslant T,
$$

indexed by $T \in \mathbb{N}$ that all depend on the common operators $B_{u}$ indexed by rescaled time $u=t / T$. Consequently, we in fact examine a triangular array of random functions that share common dynamics as provided by the continuous operator-valued function $B_{u}, u=\frac{t}{T} \in[0,1]$. For each $T$, a different 'level' of the sequence is thus considered where the dynamics change more slowly for increasing values of $T$. We will establish a class of functional time series with a time-varying functional spectral representation that includes interesting processes such as the above example and higher order time-varying functional ARMA models. The framework as provided in this chapter will allow to investigate how nonstationarity affects existing methods, such as (dynamic) functional principal component analysis (see Panaretos and Tavakoli, 2013a; Hörmann et al., 2015), and how these methods should be adjusted in order to be robust for changing characteristics. Similarly as Dahlhaus and Subba Rao (2006) and Vogt (2012) in the case of ordinary time series, we call a functional time series locally stationary if it can be locally approximated by a stationary functional time series.

Definition 3.2.1 (Local stationarity). A stochastic processes $X_{t, T} \in H$ with $t=$ $1, . ., T$ and $T \in \mathbb{N}$ is called locally stationary if for all rescaled times $u \in[0,1]$ there 
exists a strictly stationary process $X_{t}^{(u)} \in H, t \in \mathbb{Z}$, such that

$$
\left\|X_{t, T}-X_{t}^{(u)}\right\|_{2} \leqslant\left(\left|\frac{t}{T}-u\right|+\frac{1}{T}\right) P_{t, T}^{(u)} \quad \text { almost surely }
$$

where $P_{t, T}^{(u)}$ is a positive real-valued process such that for some $\rho>0$ and $C<\infty$ the process satisfies $\mathbb{E}\left(\left|P_{t, T}^{(u)}\right|^{\rho}\right)<C$ for all $t$ and $T$ and uniformly in $u \in[0,1]$.

The above definition will be further investigated in Aue and van Delft (2016). For the remainder of the chapter, we will focus on frequency domain-based methods. For this we provide sufficient conditions for local stationarity in terms of spectral representations. We start by providing the necessary terminology on operators and spectral representations for stationary functional time series.

\subsubsection{Functional spaces and operators}

First, we introduce some basic notation and definitions on functional spaces and operators. Let $L^{2}(\Omega, H, \mathbb{P})$ be the Hilbert space of $H$-valued random variables with finite second moment where $\mathbb{P}$ is the underlying probability measure and $H=$ $L^{2}\left([0,1], \mathscr{B}_{[0,1]}, \lambda\right)$, with $\lambda$ indicating the Lebesgue measure, is the separable Hilbert space of all complex-valued square integrable functions defined on $[0,1]$. To ease notation the latter space will sometimes be abbreviated by $H=L^{2}([0,1], \mathbb{C})$. The main object of the current chapter are functional time series $X=\left\{X_{t}\right\}_{t \in \mathbb{Z}}$, where $X_{t}$ takes values in the Hilbert space $H_{\mathbb{R}}=L^{2}([0,1], \mathbb{R})$ and we shall therefore denote $\mathbb{H}=L^{2}\left(\Omega, H_{\mathbb{R}}, \mathbb{P}\right)$ to be the Hilbert space of $H_{\mathbb{R}}$-valued random variables. We remark that the interval $[0,1]$ is chosen for simplicity, but the results of this chapter hold for any separable Hilbert space.

The complex conjugate of $z \in \mathbb{C}$ is denoted by $\bar{z}$ and the imaginary number by i. Then the inner product and the induced norm on $L^{2}([0,1], \mathbb{C})$ are as usual given by

$$
\langle f, g\rangle=\int_{0}^{1} f(\tau) \overline{g(\tau)} d \tau \quad \text { and } \quad\|f\|_{2}=\sqrt{\langle f, f\rangle}
$$

respectively, for $f, g \in L^{2}([0,1], \mathbb{C})$. More generally, for a function $g \in L^{2}\left([0,1]^{p}, \mathbb{C}\right)$ we shall denote the $L^{p}$-norm and the supremum norm by

$\|g\|_{p}=\left(\int_{[0,1]^{k}}\left|g\left(\tau_{1}, \ldots, \tau_{k}\right)\right|^{p} d \tau_{1} \cdots d \tau_{k}\right)^{1 / p} \quad$ and $\quad\|g\|_{\infty}=\sup _{\tau_{1}, . ., \tau_{k} \in[0,1]}\left|g\left(\tau_{1}, \ldots, \tau_{k}\right)\right|$, respectively.

Next, an operator $A$ on $H$ is said to be compact if there exist orthonormal bases $\left\{\phi_{n}\right\}$ and $\left\{\psi_{n}\right\}$ of $H$ and a monotonically decreasing sequence of non-negative numbers $s_{n}(A), n \in \mathbb{N}$ converging to zero, such that

$$
A=\sum_{n=1}^{\infty} s_{n}(A) \psi_{n} \otimes \phi_{n}
$$


where $\otimes$ denotes the tensor product. The values $s_{n}(A)$ are called the singular values of $A$ and (4.2) is the singular value decomposition of $A$. For operators on $H$, we denote the Schatten $p$-class by $S_{p}(H)$ and its norm by $\|\cdot\|_{p}$. More specifically, for $p=\infty$, the space $S_{\infty}(H)$ indicates the space of bounded linear operators equipped with the standard operator norm, while for $1 \leqslant p<\infty$ the Schatten $p$-class is the subspace of all compact operators $A$ on $H$ such that the sequence $s(A)=\left(s_{n}(A)\right)_{n \in \mathbb{N}}$ of singular values of $A$ belongs to $\ell^{p}$; the corresponding norm is given by $\|A\|_{p}^{n}=$ $\|s(A)\|_{p}$. For $1 \leqslant p \leqslant q \leqslant \infty$, we have the inclusion $S_{p}(H) \subseteq S_{q}(H)$. Two important classes are the Trace-class (nuclear) and the Hilbert-Schmidt operators on $H$, which are given by $S_{1}(H)$ and $S_{2}(H)$, respectively. More properties of Schatten class operators and in particular of Hilbert-Schmidt operators are provided in Appendix A.1. Finally, the adjoint of $A$ is denoted by $A^{\dagger}$ while the identity and zero operator are given by $I_{H}$ and $O_{H}$, respectively.

\subsubsection{Cramér representation for strictly stationary processes}

We recall some basic properties of functional time series. First, a functional time series $X=\left\{X_{t}\right\}_{t \in \mathbb{Z}}$ is called strictly stationary if, for all finite sets of indices $J \subset \mathbb{Z}$, the joint distribution of $\left\{X_{t+j} \mid j \in J\right\}$ does not depend on $t \in \mathbb{Z}$. Similarly, $X$ is weakly stationary if its first- and second-order moments exist and are invariant under translation in time. In that case, we define the mean function of $X$ by $m(\tau)=\mathbb{E}\left(X_{t}(\tau)\right)$ for $\tau \in[0,1]$ and the autocovariance kernel at lag $h \in \mathbb{Z}$ by

$$
c_{h}(\tau, \sigma)=\operatorname{cov}\left(X_{t+h}(\tau), X_{t}(\sigma)\right), \quad \tau, \sigma \in[0,1] ;
$$

both are well defined in the $L^{2}$ sense if $\mathbb{E}\left\|X_{0}\right\|_{2}^{2}<\infty$. By Proposition A.1.7, each kernel $c_{h}$ induces a corresponding autocovariance operator $\mathcal{C}_{h}$ on $H_{\mathbb{R}}$ by

$$
\mathcal{C}_{h} g(\tau)=\int_{0}^{1} c_{h}(\tau, \sigma) g(\sigma) d \sigma=\mathbb{E}\left(\left\langle g, X_{0}\right\rangle X_{h}(\tau)\right)
$$

for all $g \in H_{\mathbb{R}}$. Because $S_{2}(H)$ is isomorphic to the tensor product, we shall sometimes refer to $\mathcal{C}_{h}$ as the autocovariance tensor. In analogy to weakly stationary multivariate time series, where the covariance matrix and spectral density matrix form a Fourier pair, the spectral density operator or tensor $\mathcal{F}_{\omega}$ is given by the Fourier transform of $\mathcal{C}_{h}$,

$$
\mathcal{F}_{\omega}=\frac{1}{2 \pi} \sum_{h \in \mathbb{Z}} \mathcal{C}_{h} e^{-\mathrm{i} \omega h}
$$

A sufficient condition for the existence of $\mathcal{F}_{\omega}$ in $S_{p}(H)$ is $\sum_{h \in \mathbb{Z}}\left\|\mathcal{C}_{h}\right\|_{p}<\infty$. Panaretos and Tavakoli (2013a) showed under the condition $\sum_{h \in \mathbb{Z}}\left\|\mathcal{C}_{h}\right\|_{1}<\infty$ that a zero-mean weakly stationary functional time series $X$ admits a functional spectral representation of the form

$$
X_{t}=\int_{-\pi}^{\pi} e^{\mathrm{i} \omega t} d Z_{\omega} \quad \text { a.s. a.e. }
$$


where $Z_{\omega}$ is a functional orthogonal increment process such that, for fixed $\omega, Z_{\omega}$ is a random element in $L^{2}([0,1], \mathbb{C})$ with $\mathbb{E}\left\|Z_{\omega}\right\|_{2}^{2}=\int_{-\pi}^{\omega}\left\|\mathcal{F}_{\lambda}\right\|_{1} d \lambda$. The representation (3.3) can thus be seen as the natural extension of the Cramér representation for weakly stationary multivariate time series (Cramér, 1942) to the infinite-dimensional case.

Since the setting of this chapter allows for higher order dependence among the functional observations, we also require the notion of higher order cumulant tensors. We define this formally in Appendix A.2. In particular, we can define the point-wise $k$-th order cumulant kernel of the process $X$ is given by

$$
c_{t_{1}, \ldots, t_{k-1}}\left(\tau_{1}, \ldots \tau_{k}\right)=\sum_{\nu=\left(\nu_{1}, . ., \nu_{p}\right)}(-1)^{p-1}(p-1) ! \prod_{l=1}^{p} \mathbb{E}\left[\prod_{j \in \nu_{l}} X_{t_{j}}\left(\tau_{j}\right)\right],
$$

where the summation is over all unordered partitions of $\{1, \ldots, k\}$. We shall refer to (3.4) as the $k$-th order cumulant kernel if it is properly defined in an $L^{2}$ sense. A sufficient condition for this to be satisfied is $\mathbb{E}\left\|X_{0}\right\|_{2}^{k}<\infty$. The cumulant kernel $c_{t_{1}, \ldots, t_{k-1}}\left(\tau_{1}, \ldots \tau_{k}\right)$ induces a $k$-th order cumulant tensor $\mathcal{C}_{t_{1}, . ., t_{k-1}}$

$$
\mathcal{C}_{t_{1}, \ldots, t_{k-1}}=\sum_{i_{1}, \ldots, i_{k} \in \mathbb{N}} \int_{[0,1]^{k}} c_{t_{1}, \ldots, t_{k-1}}\left(\tau_{1}, \ldots \tau_{k}\right) \prod_{j=1}^{k} \psi_{i_{j}}\left(\tau_{j}\right) d \tau_{1} \cdots d \tau_{k} \psi_{i_{1}} \otimes \cdots \otimes \psi_{i_{k}},
$$

where $\left\{\psi_{i}\right\}_{i \in \mathbb{N}}$ is an orthonormal basis of $H$. Similar to the second-order case, this operator will form a Fourier pair with a $2 k$-th order cumulant spectral operator given summability with respect to $\|\cdot\|_{p}$ is satisfied. The $2 k$-th order cumulant spectral operator is specified as

$$
\mathcal{F}_{\omega_{1}, . ., \omega_{2 k-1}}=(2 \pi)^{1-2 k} \sum_{t_{1}, . ., t_{2 k-1} \in \mathbb{Z}} \mathcal{C}_{t_{1}, . ., t_{2 k-1}} \exp \left(-\mathrm{i} \sum_{j=1}^{2 k-1} \omega_{j} t_{j}\right)
$$

where the convergence is in $\|\cdot \cdot\|_{p}$. Note that for $k>1$, it maps onto $L^{2}\left([0,1]^{k}, \mathbb{C}\right)$. Under suitable regularity conditions, the corresponding kernels also form a Fourier pair as shown in section A.2 of the appendix. More properties on the kernels that are relevant in our framework are discussed in section 3.3. We moreover require the following theorem, which is similar to Theorem 4.6.1 of Brillinger (1981). For this, let $\eta(\lambda)=\sum_{j=-\infty}^{\infty} \delta(\lambda+2 \pi j)$ be the $2 \pi$-periodic extension of the Dirac delta function. The proof of the theorem can be found in Appendix A.4.1.

Theorem 3.2.2. Let $\left\{X_{t}\right\}_{t \in \mathbb{Z}}$ be a strictly stationary stochastic process in $L^{2}([0,1], \mathbb{R})$ such that $\mathbb{E}\left\|X_{0}\right\|_{2}^{k}<\infty$ for all $k \in \mathbb{N}$ and $\sum_{t_{1}, \ldots, t_{k-1}=-\infty}^{\infty}\left\|c_{t_{1}, \ldots, t_{k-1}}\right\|_{2}<\infty$. Furthermore let

$$
Z_{\omega}^{(N)}(\tau)=\frac{1}{2 \pi} \sum_{t=-N}^{N} X_{t}(\tau) \int_{-\pi}^{\omega} e^{-\mathrm{i} \lambda t} d \lambda
$$

Then there exists a $2 \pi$-periodic stochastic process $\left\{Z_{\omega}\right\}_{\omega \in \mathbb{R}}$ taking values in $L^{2}([0,1], \mathbb{C})$ with $\bar{Z}_{\omega}=Z_{-\omega}$ such that $\lim _{N \rightarrow \infty} \mathbb{E}\left\|Z_{\omega}^{(N)}-Z_{\omega}\right\|_{2}^{2}=0$. Moreover, $\left\{Z_{\omega}\right\}$ almost surely 
and almost everywhere equals the functional orthogonal increment process of the Cramer representation of $\left\{X_{t}\right\}$, that is,

$$
X_{t}=\int_{-\pi}^{\pi} e^{\mathrm{i} \omega t} d Z_{\omega} \quad \text { a.s and a.e. }
$$

Finally, we have for $k \geqslant 2$

$$
\begin{aligned}
\operatorname{cum}\left(Z_{\omega_{1}}\left(\tau_{1}\right), \ldots, Z_{\omega_{k}}\left(\tau_{k}\right)\right)= & \int_{-\pi}^{\omega_{1}} \ldots \int_{-\pi}^{\omega_{k}} \eta\left(\alpha_{1}+\ldots+\alpha_{k}\right) \\
& \times f_{\alpha_{1}, \ldots, \alpha_{k-1}}\left(\tau_{1}, \ldots, \tau_{k}\right) d \alpha_{1} \cdots d \alpha_{k},
\end{aligned}
$$

which holds almost everywhere and in $L^{2}$. We have in differential notation,

$$
\operatorname{cum}\left(d Z_{\omega_{1}}\left(\tau_{1}\right), \ldots, d Z_{\omega_{k}}\left(\tau_{k}\right)\right)=\eta\left(\omega_{1}+\ldots+\omega_{k}\right) f_{\omega_{1}, \ldots, \omega_{k-1}}\left(\tau_{1}, \ldots, \tau_{k}\right) d \omega_{1} \cdots d \omega_{k} .
$$

Throughout the rest of the chapter, time points in $\{1, \ldots, T\}$ will be denoted by $t, s$ or $r$, while rescaled time points on the interval $[0,1]$ will be given by $u$ and $v$. Additionally, angular frequencies are indicated with $\lambda, \alpha, \beta$ or $\omega$ and functional arguments are denoted by $\tau, \sigma$ or $\mu$.

\subsubsection{Existence of stochastic integral}

Before we provide sufficient conditions for local stationarity of functional processes in terms of spectral representations, we turn to the conditions under which stochastic integrals $\int_{-\pi}^{\pi} U_{\omega} d Z_{\omega}$ for $S_{\infty}(H)$-valued functions $U_{\omega}$ are well-defined. For this, let $\mu$ be a measure on the interval $[-\pi, \pi]$ given by

$$
\mu(E)=\int_{E}\left\|\mathcal{F}_{\omega}\right\|_{1} d \omega
$$

for all Borel sets $E \subseteq[-\pi, \pi]$ and let $\mathcal{B}_{\infty}=L^{2}\left([-\pi, \pi], S_{\infty}(H), \mu\right)$ be the corresponding Bochner space of all strongly measurable functions $U:[-\pi, \pi] \rightarrow S_{\infty}(H)$ such that

$$
\|U\|_{\mathcal{B}_{\infty}}^{2}=\int_{-\pi}^{\pi}\left\|U_{\omega}\right\|_{\infty}^{2} d \mu(\omega)<\infty .
$$

Panaretos and Tavakoli (2013a) showed that the stochastic integral is well defined in $\mathbb{H}_{\mathbb{C}}=L^{2}(\Omega, H, \mathbb{P})$ for operators that belong to the Bochner space $\mathcal{B}_{2}=$ $L^{2}\left([-\pi, \pi], S_{2}(H), \mu\right)$, which is a subspace of $\mathcal{B}_{\infty}$. In particular, it contains all functions $U:[-\pi, \pi] \rightarrow S_{2}(H)$ of the form

$$
U_{\omega}=g(\omega) I+A_{\omega}
$$

where $g$ and $A$ are, respectively, $\mathbb{C}$ and $S_{2}(H)$-valued functions that are both càdlàg with a finite number of jumps and $A$ additionally satisfies $\int_{-\pi}^{\pi}\left\|A_{\omega}\right\|\left\|_{2}^{2}\right\| \mathcal{F}_{\omega} \|_{1} d \omega<\infty$. Here, continuity in $S_{2}(H)$ is meant with respect to the operator norm $\|\cdot\|_{\infty}$. Because the space $\mathcal{B}_{2}$ is too restrictive to include interesting processes such as general 
functional autoregressive processes, we first show that the integral is properly defined in $\mathbb{H}_{\mathbb{C}}$ for all elements of $\mathcal{B}_{\infty}$. To do so, consider the subspace $\mathcal{Q}_{0} \subset \mathcal{B}_{\infty}$ of step functions spanned by elements $U \mathbf{1}_{[\alpha, \beta)}$ for $U \in S_{\infty}(H)$ and $\alpha<\beta \in[-\pi, \pi]$. Additionally, denote its closure by $\mathcal{Q}=\overline{\mathcal{Q}_{0}}$. Define then the mapping $\mathcal{T}: \mathcal{Q}_{0} \mapsto \mathbb{H}_{\mathbb{C}}$ by linear extension of

$$
\mathcal{T}\left(U \mathbf{1}_{[\alpha, \beta)}\right)=U\left(Z_{\beta}-Z_{\alpha}\right) .
$$

The following lemma shows that the image of $\mathcal{T}$ is in $\mathbb{H}_{\mathbb{C}}$.

Lemma 3.2.3. Let $X_{t}$ be a weakly stationary functional process with spectral representation $X_{t}=\int_{-\pi}^{\pi} e^{\mathrm{i} \omega t} d Z_{\omega}$ for some functional orthogonal increment process $Z_{\omega}$ that satisfies $\mathbb{E}\left\|Z_{\omega}\right\|_{2}^{2}=\int_{-\pi}^{\omega}\left\|\mathcal{F}_{\lambda}\right\|_{1} d \lambda$. Then for $U_{1}, U_{2} \in S_{\infty}(H)$ and $\alpha, \beta \in[-\pi, \pi]$

$$
\left\langle U_{1} Z_{\alpha}, U_{2} Z_{\beta}\right\rangle_{\mathbb{H}_{\mathbb{C}}}=\operatorname{tr}\left(U_{1}\left[\int_{-\pi}^{\alpha \wedge \beta} \mathcal{F}_{\omega} d \omega\right] U_{2}^{\dagger}\right)
$$

and

$$
\left\|U_{1} Z_{\alpha}\right\|_{\mathbb{H}_{\mathbb{C}}}^{2} \leqslant\left\|U_{1}\right\|_{\infty}^{2} \int_{-\pi}^{\alpha}\left\|\mathcal{F}_{\lambda}\right\|_{1} d \lambda
$$

Proof of Lemma 3.2.3. Firstly, we note that by Cauchy-Schwarz Inequality

$$
\begin{aligned}
\mathbb{E} \int_{0}^{1}\left|U_{1} Z_{\alpha}(\tau) U_{2} Z_{\beta}(\tau)\right| d \tau & \leqslant \mathbb{E}\left\|U_{1} Z_{\alpha}\right\|_{2}\left\|U_{2} Z_{\beta}\right\|_{2} \leqslant\left\|U_{1}\right\|_{\infty}\left\|U_{2}\right\|_{\infty} \mathbb{E}\left\|Z_{\alpha}\right\|_{2}\left\|Z_{\beta}\right\|_{2} \\
& \leqslant\left\|U_{1}\right\|\left\|_{\infty}\right\| U_{2}\|\|_{\infty} \int_{-\pi}^{\alpha \wedge \beta}\left\|\mathcal{F}_{\lambda}\right\|_{1} d \lambda<\infty
\end{aligned}
$$

Secondly, $U_{1} Z_{\alpha}$ and $U_{2} Z_{\beta}$ are elements in $H$ and therefore the (complete) tensor product $U_{1} Z_{\alpha} \otimes U_{2} \overline{Z_{\beta}}$ belongs to $S_{2}(H)$. By Proposition A.1.7, it is thus a kernel operator with kernel $\left[U_{1} Z_{\alpha} \otimes U_{2} \overline{Z_{\beta}}\right](\tau, \sigma)=U_{1} Z_{\alpha}(\tau) \overline{U_{2} Z_{\beta}}(\sigma)$. An application of Fubini's Theorem yields

$$
\begin{aligned}
& \mathbb{E} \int_{0}^{1} U_{1} Z_{\alpha}(\tau) \overline{U_{2} Z_{\beta}}(\tau) d \tau=\int_{0}^{1} E\left(U_{1} Z_{\alpha} \otimes U_{2} \overline{Z_{\beta}}\right)(\tau, \tau) d \tau \\
& \quad=\int_{0}^{1}\left(U_{1} \otimes U_{2}\right) \mathbb{E}\left(Z_{\alpha} \otimes \overline{Z_{\beta}}\right)(\tau, \tau) d \tau=\int_{0}^{1}\left(U_{1} \otimes U_{2}\right) \int_{-\pi}^{\alpha \wedge \beta} \mathcal{F}_{\omega} d \omega(\tau, \tau) d \tau \\
& \quad=\int_{0}^{1} U_{1} \int_{-\pi}^{\alpha \wedge \beta} \mathcal{F}_{\omega} d \omega(\tau, \tau) U_{2}^{\dagger} d \tau
\end{aligned}
$$

where the second equality follows because the expectation commutes with bounded operators for integrable random functions (Proposition A.2.1) and the last equality follows from the identity (3.59) of definition A.1.2. This shows the first result of Lemma 3.2.3. The second result follows straightforwardly from (3.11). 
It is easily seen from the previous lemma that for $\lambda_{1}>\lambda_{2} \geqslant \lambda_{3}>\lambda_{4}$

$$
\left\langle U_{1}\left(Z_{\lambda_{1}}-Z_{\lambda_{2}}\right), U_{2}\left(Z_{\lambda_{3}}-Z_{\lambda_{4}}\right)\right\rangle_{\mathbb{H}_{\mathbb{C}}}=0,
$$

demonstrating orthogonality of the increments is preserved. Since every element $U_{n} \in \mathcal{Q}_{0}$ can be written as $\sum_{j=1}^{n} U_{j} \mathbf{1}_{\left[\lambda_{j}, \lambda_{j+1}\right)}$ the lemma moreover implies

$$
\begin{aligned}
\|\mathcal{T}(U)\|_{\mathbb{H}_{\mathbb{C}}}^{2} & =\sum_{j, k=1}^{n}\left\langle U_{j}\left(Z_{\lambda_{j+1}}-Z_{\lambda_{j}}\right), U_{k}\left(Z_{\lambda_{k+1}}-Z_{\lambda_{k}}\right)\right\rangle_{\mathbb{H}_{\mathbb{C}}}=\sum_{j=1}^{n}\left\|U_{j}\left(Z_{\lambda_{j+1}}-Z_{\lambda_{j}}\right)\right\|_{\mathbb{H}}^{2} \\
& \leqslant \sum_{j=1}^{n}\left\|U_{j}\right\|_{\infty}^{2} \int_{\lambda_{j}}^{\lambda_{j+1}}\left\|\mathcal{F}_{\alpha}\right\|_{1} d \alpha=\|U\|_{\mathcal{B}_{\infty}}^{2} .
\end{aligned}
$$

The mapping $\mathcal{T}: \mathcal{Q}_{0} \mapsto \mathbb{H}_{\mathbb{C}}$ is therefore continuous. Together with the completeness of the space $\mathbb{H}_{\mathbb{C}}$ this establishes that, for every sequence $\left\{U_{n}\right\}_{n \geqslant 1} \subset \mathcal{Q}_{0}$ converging to some element $U \in \overline{\mathcal{Q}}$, the sequence $\left\{\mathcal{T}\left(U_{n}\right)\right\}_{n \geqslant 1}$ forms a Cauchy sequence in $\mathbb{H}_{\mathbb{C}}$ with limit $\mathcal{T}(U)=\lim _{n \rightarrow \infty} \mathcal{T}\left(U_{n}\right)$. By linearity and continuity of the mapping $\mathcal{T}$, the limit is independent of the choice of the sequence. Furthermore, since $\mathcal{Q}_{0}$ is the subspace spanned by step functions that are square integrable on $[-\pi, \pi]$ with respect to the finite measure $\mu$ and hence is dense in $L^{2}\left([-\pi, \pi], S_{\infty}(H), \mu\right)$, we have $\mathcal{B}_{\infty} \subseteq \overline{\mathcal{Q}}$. Since $\|\mathcal{T}(U)\|_{\mathbb{H}_{\mathbb{C}}} \leqslant\|U\|_{\mathcal{B}_{\infty}}$, the above extension is well-defined for all $U \in \mathcal{B}_{\infty}$.

Because stronger results can be obtained for elements of the Bochner space $\mathcal{B}_{2}$, we will make the distinction between these elements and general elements of $\mathcal{B}_{\infty}$ throughout the rest of the chapter.

\subsubsection{Local stationarity in the frequency domain}

With the results from the previous two subsections, we can now give a characterization of locally stationary functional processes in the frequency domain. This can be viewed a generalization of Dahlhaus (1996a) to the functional setting.

Proposition 3.2.4. Let $\left\{\varepsilon_{t}\right\}_{t \in \mathbb{Z}}$ be a weakly stationary process taking values in $H_{\mathbb{R}}$ with spectral representation $\varepsilon_{t}=\int_{-\pi}^{\pi} e^{-\mathrm{i} \omega t} d Z_{\omega}$. Furthermore, suppose that the functional process $X_{t, T}$ with $t=1, \ldots, T$ and $T \in \mathbb{N}$ is given by

$$
X_{t, T}=\int_{-\pi}^{\pi} e^{\mathrm{i} \omega t} \mathcal{A}_{t, \omega}^{(T)} d Z_{\omega} \quad \text { a.s. a.e. }
$$

with transfer operator $\mathcal{A}_{t, \omega}^{(T)} \in \mathcal{B}_{p}$ for $p \in\{2, \infty\}$. If there exists an $S_{p}(H)$-valued function $\mathcal{A}_{u, \omega}$ that is $2 \pi$-periodic with respect to $\omega$ and continuous in $u \in[0,1]$ such that for all $T \in \mathbb{N}$

$$
\sup _{\omega, t}\left\|\mathcal{A}_{t, \omega}^{(T)}-\mathcal{A}_{\frac{t}{T}, \omega}\right\|_{p}=O\left(\frac{1}{T}\right)
$$

then $\left\{X_{t, T}\right\}$ is a locally stationary process in $H_{\mathbb{R}}$. 
Proof. For $u \in[0,1]$, we define the approximating stationary functional process $\left\{X_{t}^{(u)}\right\}_{t \in \mathbb{Z}}$ by

$$
X_{t}^{(u)}=\int_{-\pi}^{\pi} e^{\mathrm{i} \omega t} \mathcal{A}_{u, \omega} d Z_{\omega}
$$

Then we have

$$
\left\|X_{t, T}-X_{t}^{(u)}\right\|_{2}=\left\|\int_{-\pi}^{\pi} e^{\mathrm{i} \omega t}\left(\mathcal{A}_{t, \omega}^{(T)}-\mathcal{A}_{u, \omega}\right) d Z_{\omega}\right\|_{2} \leqslant c P_{t, T}^{(u)}
$$

with

$$
\begin{aligned}
c & =\sup _{\omega}\left\|\mathcal{A}_{t, \omega}^{(T)}-\mathcal{A}_{u, \omega}\right\|_{p} \\
& \leqslant \sup _{\omega}\left\|\mathcal{A}_{t, \omega}^{(T)}-\mathcal{A}_{t / T, \omega}\right\|_{p}+\sup _{\omega}\left\|\mathcal{A}_{t / T, \omega}-\mathcal{A}_{u, \omega}\right\|_{p}=O\left(\frac{t}{T}+\left|\frac{t}{T}-u\right|\right)
\end{aligned}
$$

and

Since

$$
P_{t, T}^{(u)}=\frac{1}{c}\left\|\int_{-\pi}^{\pi} e^{\mathrm{i} \omega t}\left(\mathcal{A}_{t, \omega}^{(T)}-\mathcal{A}_{u, \omega}\right) d Z_{\omega}\right\|_{2}
$$

$$
\mathbb{E}\left|P_{t, T}^{(u)}\right|^{2} \leqslant \frac{1}{c^{2}} \int_{-\pi}^{\pi}\left\|\mid \mathcal{A}_{t, \omega}^{(T)}-\mathcal{A}_{u, \omega}\right\|_{\infty}^{2}\left\|\mathcal{F}_{\omega}\right\|_{1} d \omega \leqslant \int_{-\pi}^{\pi}\left\|\mathcal{F}_{\omega}\right\|_{1} d \omega,
$$

the process satisfies the conditions of Definition 3.2.1 with $\rho=2$.

As in the time series setting, we need the existence of a transfer operator $\mathcal{A}_{u, \omega}$ that is continuous in $u \in[0,1]$ to guarantee locally an approximately stationary behavior without sudden changes. In order to include interesting cases such as autoregressive processes for which a time-varying functional spectral representation with a common continuous transfer operator $\mathcal{A}_{u, \omega}$ does not exist, we require that such a representation only holds approximately by condition (ii) of Proposition 3.2.4. We remark that Proposition 3.2.4 will coincide with the original characterization (Dahlhaus, 1996a) if the data are in fact finite-dimensional.

It follows directly from Panaretos and Tavakoli (2013a) that a spectral representation with transfer operator $\mathcal{A}_{t, \omega}^{(T)}=\frac{1}{\sqrt{2 \pi}} \sum_{s=-\infty}^{\infty} A_{t, s}^{(T)} e^{-\mathrm{i} \omega s}$ exists for time-varying processes of the form

$$
X_{t, T}=\sum_{s \in \mathbb{Z}} A_{t, s}^{(T)} \varepsilon_{t-s}
$$

where $\left\{\varepsilon_{s}\right\}_{s \in \mathbb{Z}}$ is a functional white noise process in $\mathbb{H}$ and $\left\{A_{t, s}^{(T)}\right\}_{s \in \mathbb{Z}}$ are sequences of linear operators in $S_{2}(H)$ satisfying $\Sigma_{s}\left\|A_{t, s}^{(T)}\right\|_{2}<\infty$ for all $t=1, \ldots, T$ and $T \in \mathbb{N}$. In order to allow for time-varying functional autoregressive processes, it will be necessary (see Section 3.4) to weaken these assumptions to linear processes where at least $A_{t, 0}^{(T)}=c I_{H}$ for some finite constant $c$. We note that this case is captured by Proposition 3.2.4 if $\mathcal{A}_{t, \omega}^{(T)} \in \mathcal{B}_{2}$. The case where a moving average representation exists with $\sum_{s}\left\|A_{t, s}^{(T)}\right\|_{\infty}<\infty$ for all $t=1, \ldots, T$ and $T \in \mathbb{N}$ will be covered by the more general set of transfer operators of the form $\mathcal{A}_{t, \omega}^{(T)} \in \mathcal{B}_{\infty}$ as shown in the following proposition. 
Proposition 3.2.5. Let $\left\{\varepsilon_{t}\right\}_{t \in \mathbb{Z}}$ be a weakly stationary process in $H_{\mathbb{R}}$ with spectral representation $\varepsilon_{t}=\int_{-\pi}^{\pi} e^{-\mathrm{i} \omega t} d Z_{\omega}$ and let $\left\{A_{t, s}^{(T)}\right\}_{s \in \mathbb{Z}}$ be a sequence of operators in $S_{\infty}(H)$ satisfying $\sum_{s}\left\|A_{t, s}^{(T)}\right\|_{\infty}<\infty$ for all $t=1, \ldots, T$ and $T \in \mathbb{N}$. Then the process

$$
X_{t, T}=\sum_{s \in \mathbb{Z}} A_{t, s}^{(T)} \varepsilon_{t-s}
$$

has a representation as given by Proposition 3.2.4 $(i)$ with $\mathcal{A}_{t, \omega}^{(T)} \in \mathcal{B}_{\infty}$.

Proof. For fixed $t \in\{1, \ldots, T\}$ and $T \in \mathbb{N}$, let $U_{s, \omega}=e^{\mathrm{i} \omega(t-s)} A_{t, s}^{(T)}$. We have

$$
A_{t, s}^{(T)} \varepsilon_{t-s}=\int_{-\pi}^{\pi} e^{\mathrm{i} \omega(t-s)} A_{t, s}^{(T)} d Z_{\omega}=\mathcal{T}\left(U_{s,}\right)
$$

where $\mathcal{T}$ is the mapping that was defined in (3.10). By definition of the operator $U_{s, \text {, }}$, $\left\|U_{s, \cdot}\right\|_{\mathcal{B}_{\infty}}^{2} \leqslant\left\|A_{t, s}^{(T)}\right\|_{\infty}^{2} \int_{-\pi}^{\pi}\left\|\mathcal{F}_{\omega}^{\varepsilon}\right\|_{1} d \omega<\infty$ and thus $U_{s, \cdot} \in \mathcal{B}_{\infty}$. Similarly, $\sum_{s} \mathcal{T}\left(U_{s, \cdot}\right) \in$ $\mathcal{B}_{\infty}$ from which it follows that

$$
\lim _{N \rightarrow \infty} \sum_{|s| \leqslant N} e^{\mathrm{i} \omega(t-s)} A_{t, s}^{(T)}=e^{\mathrm{i} \omega t} \sum_{s \in \mathbb{Z}} e^{-\mathrm{i} \omega s} A_{t, s}^{(T)}=e^{\mathrm{i} \omega t} \mathcal{A}_{t, \omega}^{(T)} \in \mathcal{B}_{\infty} .
$$

The continuity of the mapping $\mathcal{T}$ then implies

$$
X_{t, T}=\sum_{s} \mathcal{T}\left(U_{s, .}\right)=\mathcal{T}\left(\sum_{s} U_{s, \cdot}\right)=\int_{-\pi}^{\pi} e^{\mathrm{i} \omega t} \mathcal{A}_{t, \omega}^{(T)} d Z_{\omega} \quad \text { a.s. a.e. }
$$

Remark 3.2.6. If the transfer function operator does not depend on $t$ and $T$, we obtain the classical case of Bosq (2000). Moreover, we note that our setting covers the framework of Hörmann and Kokoszka (2010). More precisely, if the linear filter given by Proposition 3.2.4 $(i)$ is causal (i.e. $A_{t, s}^{(T)}=0$ for $s<0$ ), we can write $X_{t, T}$ also as $X_{t, T}=g_{t, T}\left(\varepsilon_{t}, \varepsilon_{t-1}, \ldots\right)$ for measurable functions $g_{t, T}: H_{\mathbb{R}}^{\infty} \rightarrow H_{\mathbb{R}}$ with $t=1, \ldots, T$ and $T \in \mathbb{N}$. This is easily seen since the operator $A_{t, \omega}^{(T)} \in \mathcal{B}_{\infty}$ has a welldefined Fourier transform so that an application of the Dominated Convergence Theorem yields $X_{t, T}=\sum_{s \in \mathbb{N}} A_{t, s}^{(T)} \varepsilon_{t-s}$. The context of $L_{m}^{p}$-approximability is then straightforward if one defines the $m$-dependent coupling process by

$$
X_{t, T}^{(m)}=g_{t, T}\left(\varepsilon_{t}, \ldots, \varepsilon_{t-m+1}, \varepsilon_{t-m}^{*}, \varepsilon_{t-m-1}^{(*)}, \ldots\right),
$$

where $\left\{\varepsilon_{t}^{*}\right\}$ is an independent copy of $\left\{\varepsilon_{t}\right\}$.

\subsection{Time-varying spectral density operator}

We will now introduce the time-varying spectral density operator and its properties. In particular, we will show that the uniqueness property of the time-varying spectral density established by Dahlhaus (1996a) also extends to the infinite dimension. Let 
$X_{t, T}(\tau)$ be given as in Proposition 3.2.4 with $\mathcal{A}_{t, \omega}^{(T)}=\mathcal{A}_{0, \omega}^{(T)}$ for $t<1$ and $\mathcal{A}_{t, \omega}^{(T)}=\mathcal{A}_{1, \omega}^{(T)}$ for $t>T$. For fixed $T$, the local autocovariance kernel is defined by

$$
c_{u, s}^{(T)}(\tau, \sigma)=\operatorname{cov}\left(X_{\lfloor u T-s / 2\rfloor, T}(\tau), X_{\lfloor u T+s / 2\rfloor, T}(\sigma)\right),
$$

where $\lfloor s\rfloor$ denotes the largest integer not greater than $s$. It is straightforward to derive that it is a proper element of $L^{2}\left([0,1]^{2}, \mathbb{R}\right)$, and so it induces a local autocovariance operator on $H_{\mathbb{R}}$ given by

$$
\mathcal{C}_{u, s}^{(T)}=\operatorname{cov}\left(X_{\lfloor u T-s / 2\rfloor, T}, X_{\lfloor u T+s / 2\rfloor, T}\right) .
$$

Proposition 3.3.1. Under the conditions of Proposition 3.2.4, the autocovariance kernel defined in (3.14) satisfies $\sum_{s \in \mathbb{Z}}\left\|c_{u, s}^{(T)}\right\|_{2}<\infty$.

The proof can be found in section A.4.2 of the appendix. In order for $c_{u, s}^{(T)}$ to be properly defined point-wise it is sufficient to additionally assume that the functional white noise process $\left\{\varepsilon_{t}\right\}_{t \in \mathbb{Z}}$ is mean square continuous - that is, its autocovariance kernel is continuous at $\operatorname{lag} h=0$ - and that the sequence of operators $\left\{A_{t, s}^{(T)}\right\}_{s \in \mathbb{Z}}$ for all $t=1, \ldots, T$ and $T \in \mathbb{N}$ is Hilbert-Schmidt with kernels that are continuous in their functional arguments. The process $\left\{X_{t, T}\right\}$ will then itself be mean square continuous which allows certain results such as the Karhunen-Loève representation to hold in uniform norm rather than in $L^{2}$-norm. In particular, a slight adjustment in the proof of Proposition 3.3.1 demonstrates that under these extra conditions we have $\sum_{s \in \mathbb{Z}}\left\|c_{u, s}^{(T)}\right\|_{\infty}<\infty$. It will become clear in the next section that a representation under these stronger conditions excludes time-varying functional $\operatorname{AR}(p)$ models, which is why we will not impose them. Similar to Proposition 2.1 of Panaretos and Tavakoli (2013b), it is easily shown that the Fourier transform of the autocovariance kernel, for fixed $t$ and $T$, provides a local spectral density kernel

$$
f_{u, \omega}^{(T)}(\tau, \sigma)=\frac{1}{2 \pi} \sum_{s \in \mathbb{Z}} c_{u, s}^{(T)}(\tau, \sigma) e^{-\mathrm{i} \omega s}
$$

that is well-defined. More precisely, given $\sum_{s \in \mathbb{Z}}\left\|c_{u, s}^{(T)}\right\|_{p}<\infty$ for $p=2$ or $p=\infty$, the spectral density kernel is uniformly bounded and uniformly continuous in $\omega$ with respect to $\|\cdot\|_{p}$. Additionally, the inversion formula

$$
c_{u, s}^{(T)}(\tau, \sigma)=\int_{-\pi}^{\pi} f_{u, \omega}^{(T)}(\tau, \sigma) e^{\mathrm{i} s \omega} d \omega
$$

holds in $\|\cdot\|_{p}$ for all $s, u, T, \tau$, and $\sigma$. Extension of this result to higher order cumulant spectral kernels is provided in Theorem A.2.3. For fixed $T$, the spectral density kernel in (3.16) can be seen as a functional version of the Wigner-Ville spectrum (see Martin and Flandrin, 1985). The corresponding induced Wigner-Ville spectral operator $\mathcal{F}_{u, \omega}^{(T)}$ forms moreover a Fourier pair with (3.15) and can similarly be given by

$$
\mathcal{F}_{u, \omega}^{(T)}=\frac{1}{2 \pi} \sum_{s} \mathcal{C}_{u, s}^{(T)} e^{-\mathrm{i} \omega s}
$$


This operator is $2 \pi$-periodic in $\omega$ and self-adjoint since $c_{u,-s}^{(T)}(\sigma, \tau)=c_{u, s}^{(T)}(\tau, \sigma)$, which implies $f_{u, \omega}^{\dagger(T)}(\sigma, \tau)=\overline{f_{u, \omega}^{(T)}(\tau, \sigma)}$. Here, $f^{\dagger}$ the kernel function of the adjoint operator $\mathcal{F}^{\dagger}$. Moreover, $\mathcal{F}_{\omega}^{\varepsilon}$ is nuclear by Parseval's Identity and therefore Proposition A.1.3 implies that (3.17) is also an element of $S_{1}(H)$. We will show in the following that (3.17) converges in integrated mean square to the time-varying spectral density operator defined as

$$
\mathcal{F}_{u, \omega}=\mathcal{A}_{u, \omega} \mathcal{F}_{\omega}^{\varepsilon} \mathcal{A}_{u, \omega}^{\dagger} .
$$

The time-varying spectral density operator satisfies all of the above properties and is additionally non-negative definite. This is straightforward by noting that, for every $\psi \in L^{2}([0,1], \mathbb{C})$,

$$
\left\langle\mathcal{A}_{u, \omega} \mathcal{F}_{\omega}^{\varepsilon} \mathcal{A}_{u, \omega}^{\dagger} \psi, \psi\right\rangle=\left\langle\mathcal{F}_{\omega}^{\varepsilon} \mathcal{A}_{u, \omega}^{\dagger} \psi, \mathcal{A}_{u, \omega}^{\dagger} \psi\right\rangle \geqslant 0,
$$

which is a consequence of the non-negative definiteness of $\mathcal{F}_{\omega}^{\varepsilon}$. For any two elements $\psi, \varphi$ in $L^{2}([0,1], \mathbb{C})$, one can interpret the mapping $\omega \mapsto\left\langle\psi, \mathcal{F}_{u, \omega} \varphi\right\rangle=$ $\left\langle\mathcal{F}_{u, \omega} \psi, \varphi\right\rangle \in \mathbb{C}$ to be the local cross-spectrum of the sequences $\left\{\left\langle\psi, X_{t}^{(u)}\right\rangle\right\}_{t \in \mathbb{Z}}$ and $\left\{\left\langle\varphi, X_{t}^{(u)}\right\rangle\right\}_{t \in \mathbb{Z}}$. In particular, $\omega \mapsto\left\langle\psi, \mathcal{F}_{u, \omega} \psi\right\rangle \geqslant 0$ can be interpreted as the local power spectrum of $\left\{\left\langle\psi, X_{t}^{(u)}\right\rangle\right\}_{t \in \mathbb{Z}}$ for all $u \in[0,1]$. In case a point-wise interpretation is possible, i.e., the random functions take values in $L^{\infty}([0,1], \mathbb{R})$, the mapping $\omega \mapsto f_{u, \omega}(\tau, \sigma)$ provides information on how the covariation of the sequences $\left\{X_{t}^{(u)}(\tau)\right\}_{t \in \mathbb{Z}}$ and $\left\{X_{t}^{(u)}(\sigma)\right\}_{t \in \mathbb{Z}}$ is distributed over different frequency components with $\omega \mapsto f_{u, \omega}(\tau, \tau)$ and $\omega \mapsto f_{u, \omega}(\tau, \sigma)$, respectively, denoting the local power spectrum and the local cross-spectrum. In analogy to the spectral density matrix in multivariate time series, we will show in the below that the local spectral density operator completely characterizes the limiting second-order dynamics of the family of functional processes $\left\{X_{t, T}: t=1, \ldots, T\right\}_{T \in \mathbb{N}}$.

Theorem 3.3.2. Let $\left\{X_{t, T}\right\}$ be a locally stationary process satisfying Proposition 3.2.4 and let the operator-valued function $\mathcal{A}_{u, \omega}$ be Hölder continuous of order $\alpha>$ $1 / 2$ in $u$ and $\omega$. Then, for all $u \in(0,1)$,

$$
\int_{-\pi}^{\pi}\left\|\mathcal{F}_{u, \omega}^{(T)}-\mathcal{F}_{u, \omega}\right\|_{2}^{2} d \omega=o(1)
$$

as $T \rightarrow \infty$.

Proof. By definition of the Wigner-Ville operator and Lemma 3.2.3,

$$
\begin{aligned}
\mathcal{F}_{u, \omega}^{(T)} & =\frac{1}{2 \pi} \sum_{s} \operatorname{cov}\left(\int_{-\pi}^{\pi} e^{\mathrm{i} \lambda\lfloor u T-s / 2\rfloor} \mathcal{A}_{\lfloor u T-s / 2\rfloor, \lambda}^{(T)} d Z_{\lambda}, \int_{-\pi}^{\pi} e^{\mathrm{i} \beta\lfloor u T+s / 2\rfloor} \mathcal{A}_{\lfloor u T+s / 2\rfloor, \beta}^{(T)} d Z_{\beta}\right) e^{-\mathrm{i} \omega s} \\
& =\frac{1}{2 \pi} \sum_{s} \int_{-\pi}^{\pi} e^{\mathrm{i} \lambda s} \mathcal{A}_{\lfloor u T-s / 2\rfloor, \lambda}^{(T)} \mathcal{F}_{\lambda}^{\varepsilon}\left(\mathcal{A}_{\lfloor u T+s / 2\rfloor, \lambda}^{(T)}\right)^{\dagger} d \lambda e^{-\mathrm{i} \omega s} .
\end{aligned}
$$

Using identity (3.59), we have that

$$
\mathcal{A}_{\lfloor u T-s / 2\rfloor, \lambda}^{(T)} \mathcal{F}_{\lambda}^{\varepsilon}\left(\mathcal{A}_{\lfloor u T+s / 2\rfloor, \lambda}^{(T)}\right)^{\dagger}=\left(\mathcal{A}_{\lfloor u T-s / 2\rfloor, \lambda}^{(T)} \otimes \mathcal{A}_{\lfloor u T+s / 2\rfloor, \lambda}^{(T)}\right) \mathcal{F}_{\lambda}^{\varepsilon} .
$$


Similarly,

$$
\mathcal{F}_{u, \omega}=\frac{1}{2 \pi} \sum_{s} \int_{-\pi}^{\pi} e^{\mathrm{i} \lambda s}\left(\mathcal{A}_{u, \lambda} \otimes \mathcal{A}_{u, \lambda}^{\dagger}\right) \mathcal{F}_{\lambda}^{\varepsilon} d \lambda e^{-\mathrm{i} \omega s} .
$$

We can therefore write the left-hand side of (3.19) as

$$
\int_{-\pi}^{\pi}\left\|\frac{1}{2 \pi} \sum_{s} \int_{-\pi}^{\pi} e^{\mathrm{i} \lambda s}\left(\mathcal{A}_{\lfloor u T-s / 2], \lambda}^{(T)} \otimes \mathcal{A}_{\lfloor u T+s / 2], \lambda}^{(T)}-\mathcal{A}_{u, \lambda} \otimes \mathcal{A}_{u, \lambda}\right) \mathcal{F}_{\lambda}^{\varepsilon} d \lambda e^{-\mathrm{i} \omega s}\right\|_{2}^{2} d \omega .
$$

Consider the operator

$$
G_{s, \lambda}^{(u, T)}=\left(\mathcal{A}_{\lfloor u T-s / 2\rfloor, \lambda}^{(T)} \otimes \mathcal{A}_{\lfloor u T+s / 2\rfloor, \lambda}^{(T)}-\mathcal{A}_{u, \lambda} \otimes \mathcal{A}_{u, \lambda}\right) \mathcal{F}_{\lambda}^{\varepsilon}
$$

and its continuous counterpart

$$
G_{\frac{s}{2 T}, \lambda}=\left(\mathcal{A}_{\left(u-\frac{s}{2 T}\right), \lambda} \otimes \mathcal{A}_{\left(u+\frac{s}{2 T}\right), \lambda}-\mathcal{A}_{u, \lambda} \otimes \mathcal{A}_{u, \lambda}\right) \mathcal{F}_{\lambda}^{\varepsilon}
$$

By Hölder's Inequality for operators (Proposition A.1.3), both are nuclear and hence Hilbert-Schmidt. Another application of Hölder's Inequality together with condition (ii) of Proposition 3.2.4 yields

$$
\begin{aligned}
& \left\|\left(\mathcal{A}_{\lfloor u T \mp s / 2\rfloor, \lambda}^{(T)} \otimes\left[\mathcal{A}_{\lfloor u T \pm s / 2\rfloor, \lambda}^{(T)}-\mathcal{A}_{u \pm \frac{s}{2 T}, \lambda}\right]\right) \mathcal{F}_{\lambda}^{\varepsilon}\right\|_{2}^{2} \\
& \leqslant\left\|\mathcal{A}_{\lfloor u T \mp s / 2\rfloor, \lambda}^{(T)}\right\|\left\|_{\infty}^{2}\right\| \mathcal{A}_{\lfloor u T \pm s / 2\rfloor, \lambda}^{(T)}-\mathcal{A}_{u \pm \frac{s}{2 T}, \lambda}\left\|_{\infty}^{2}\right\| \mathcal{F}_{\omega}^{\varepsilon} \|_{2}^{2}=O\left(\frac{1}{T^{2}}\right) .
\end{aligned}
$$

Minkowski's Inequality then implies

$$
\int_{-\pi}^{\pi}\left\|\mathcal{F}_{u, \omega}^{(T)}-\mathcal{F}_{u, \omega}\right\|_{2}^{2} d \omega=\int_{-\pi}^{\pi}\left\|G_{\frac{s}{2 T}, \omega}\right\|_{2}^{2} d \omega+o(1)
$$

It is therefore sufficient to derive a bound on

$$
\int_{-\pi}^{\pi}\left\|G_{\frac{s}{2 T}, \omega}\right\|_{2}^{2} d \omega
$$

A similar argument as in (3.20) shows that

$$
\left\|\left(\mathcal{A}_{\left(u-\frac{s}{2 T}\right), \omega} \otimes\left(\mathcal{A}_{\left(u+\frac{s}{2 T}\right), \omega}-\mathcal{A}_{u, \omega}\right)+\left(\mathcal{A}_{\left(u-\frac{s}{2 T}\right), \omega}-\mathcal{A}_{u, \omega}\right) \otimes \mathcal{A}_{u, \omega}\right) \mathcal{F}_{\omega}^{\varepsilon}\right\|_{2} \leqslant C\left|\frac{s}{2 T}\right|^{\alpha}
$$

for some constant $C>0$. The operator-valued function $G_{u, \omega}$ is therefore Hölder continuous of order $\alpha>1 / 2$ in $u$. Using the inversion formula (Theorem A.2.3) consecutively, we can write (3.21) as

$$
\begin{aligned}
& \frac{1}{(2 \pi)^{2}} \int_{-\pi}^{\pi} \sum_{s, s^{\prime}} e^{-\mathrm{i} \omega\left(s-s^{\prime}\right)}\left\langle\int_{0}^{2 \pi} G_{\frac{s}{2 T}, \lambda}, e^{\mathrm{i} s \lambda} d \lambda, \int_{0}^{2 \pi} \overline{G_{\frac{s^{\prime}}{2 T}, \lambda^{\prime}}} e^{-\mathrm{i} s^{\prime} \lambda^{\prime}} d \lambda^{\prime}\right\rangle_{H \otimes H} d \omega \\
& =\frac{1}{2 \pi} \sum_{s \in \mathbb{Z}}\left\|\tilde{G}_{\frac{s}{2 T}}\right\|_{2}^{2}
\end{aligned}
$$


where $\tilde{G}_{s}$ can be viewed as the $s$-th Fourier coefficient operator of $G_{\frac{s}{2 T}, \lambda}$. Because of Hölder continuity, we have that these satisfy $\left\|\tilde{G}_{s}\right\|_{2} \leqslant\left.\left\|\pi^{\alpha+1}\right\| G_{u, \omega}\left|\|_{2}\right| s\right|^{-\alpha}=O\left(s^{-\alpha}\right)$. Hence,

$$
\sum_{s=n}^{\infty}\left\|\tilde{G}_{\frac{|s|}{2 T}}\right\|_{2}^{2}=O\left(n^{1-2 \alpha}\right) .
$$

Concerning the partial sum $\sum_{s=0}^{n-1}\left|\hat{g}_{s}(\tau, \sigma)\right|^{2}$, we proceed as in Dahlhaus (1996a) and use summation by parts to obtain

$$
\sum_{s=0}^{n-1}\left\|\tilde{G}_{s}\right\|_{2}^{2}=\int_{0}^{2 \pi} \int_{0}^{2 \pi} \sum_{s=0}^{n-1} e^{\mathrm{i} s\left(\lambda-\lambda^{\prime}\right)}\left\langle G_{\frac{s}{2 T}, \lambda}, \overline{G_{\frac{s^{\prime}}{2 T}, \lambda^{\prime}}}\right\rangle_{H \otimes H} d \lambda d \lambda^{\prime}=O\left(\frac{n \log (n)}{T^{\alpha}}\right)
$$

which follow from the properties of $\tilde{G}_{s}$ and Lemma A.3.1. It is straightforward to see that $\sum_{s=0}^{n-1}\left|\tilde{G}_{-s}\right|^{2}$ satisfies the same bound. Hence,

$$
\begin{aligned}
\int_{-\pi}^{\pi}\left\|\mathcal{F}_{u, \omega}^{(T)}-\mathcal{F}_{u, \omega}\right\|_{2}^{2} d \omega & =\int_{-\pi}^{\pi}\left\|G_{\frac{s}{2 T}, \omega}\right\|_{2}^{2} d \omega+o(1) \\
& =O\left(n^{1-2 \alpha}\right)+O\left(\frac{n \log (n)}{T^{\alpha}}\right) .
\end{aligned}
$$

Choosing an appropriate value $n \ll T$ completes the proof.

It is well-known from the time series setting that a Cramér representation as given in Proposition 3.2.4 is in general not unique (e.g. Priestley, 1981). However, Theorem 3.3.2 shows that the uniqueness property as proved by Dahlhaus (1996a) generalizes to the functional setting. That is, if the family of functional processes $\left\{X_{t, T}: t=1, \ldots, T\right\}_{T \in \mathbb{N}}$ taking values in $H$ has a representation with common transfer operator $\mathcal{A}_{u, \omega}$ that operates on this space and that is continuous in $u$, then the time-varying spectral density operator will be uniquely determined from the triangular array.

Intuitively, the value of $n$ such that $n \log (n) T^{-\alpha} \rightarrow 0$ can be seen to determine the length of the data-segment over which the observations are approximately stationary. To see this, observe that only those functional observations $X_{t, T}$ from the triangular array with $t / T \in\left[u+\frac{n}{T}, u-\frac{n}{T}\right]$ will effectively contribute to the time-varying spectral density operator at $u$. As $T$ increases, the width of this interval shrinks and sampling becomes more dense. Because the array shares dynamics through the operator-valued function $\mathcal{A}_{u, \omega}$, which is smooth in $u$, the observations belonging to this interval will thus become close to stationary as $T \rightarrow \infty$. The theorem therefore implies that, if we would have infinitely many observations with the same probabilistic structure around some fixed time point, the local second-order dynamics of the family are uniquely characterized by the operator $\mathcal{F}_{u, \omega}$.

Remark 3.3.3. Note that in case Proposition 3.2.4 holds with $p=2$, we have by continuity of the inner product that the kernel $a_{u, \omega} \in L^{2}\left([0,1]^{2}, \mathbb{C}\right)$ of $\mathcal{A}_{u, \omega}$ is uniformly Hölder continuous of order $\alpha>1 / 2$ in both $u$ and $\omega$. If we thus additionally assume 
that the $\left\{\varepsilon_{t}\right\}_{t \in \mathbb{Z}}$ are mean square continuous and the operator $\mathcal{A}_{u, \omega}$ is an element of $\mathcal{B}_{2}$ of which the Hilbert-Schmidt component has a kernel that is continuous in its functional arguments, the error holds in uniform norm.

\subsection{Locally stationary functional autoregressive processes}

Autoregressive processes are of general interest as they have applications in a wide range of disciplines such as economics and medicine and can especially be useful for prediction purposes. Early work on prediction based on the functional autoregressive mode can for example be found in Damon and Guillas (1982), Besse and Ramsay (1986) and Antoniadis and Sapatinas (2003). Linear processes in Hilbert and Banach spaces and in particular functional autoregressive processes have also been thoroughly investigated in the monograph of Bosq (2000). Although the model of Bosq (2000) assumes only that the errors of the causal solution in an appropriate Hilbert space sense are uncorrelated, nowadays most estimation techniques are still based on the assumption of i.i.d. functional errors. In order to incorporate temporal dependence among functional observations, Hörmann and Kokoszka (2010) introduced a moment-based notion of dependence, $L_{m}^{p}$-approximability, and investigated the properties of the functional $\mathrm{AR}(1)$ under the new set of conditions. We will now show that time-varying functional autoregressive processes are locally stationary in the sense of Proposition 3.2.4 and that stationary functional $\operatorname{AR}(p)$ are a special case. First, we will have to show that a causal solution exists for these type of processes. This is done in the theorem stated below.

Theorem 3.4.1. Let $\left\{\varepsilon_{t}\right\}_{t \in \mathbb{Z}}$ be a white noise process in $H_{\mathbb{R}}$. Then the locally stationary functional $A R(p)$ process

$$
X_{t, T}=\sum_{j=1}^{p} B_{\frac{t}{T}, j}\left(X_{t-j, T}\right)+\varepsilon_{t}
$$

will have a unique causal solution of the form

$$
X_{t, T}(\tau)=\sum_{l=0}^{\infty} A_{t, l}^{(T)}\left(\varepsilon_{t-l}\right)(\tau)
$$

with $\sup _{t, T} \sum_{l=0}^{\infty}\left\|A_{t, l}^{(T)}\right\|_{\infty}<\infty$ if

(i) the operators $B_{u, j}$ are continuous in $u \in[0,1]$ for all $j=1, \ldots, p$;

(ii) for all $u \in[0,1]$, the operators satisfy $\sum_{j=1}^{p}\left\|B_{u, j}\right\|_{\infty}<1$. 
In order to prove the theorem, note that we can represent the functional $A R(p)$ process in state space form

$$
\underbrace{\left(\begin{array}{c}
X_{t, T} \\
X_{t-1, T} \\
\vdots \\
X_{t-p+1, T}
\end{array}\right)}_{\boldsymbol{X}_{t, T}^{*}}=\underbrace{\left(\begin{array}{cccc}
B_{\frac{t}{T}, 1} & B_{\frac{t}{T}, 2} & \cdots & B_{\frac{t}{T}, p} \\
I_{H} & & & O_{H} \\
& \ddots & & \vdots \\
& & I_{H} & O_{H}
\end{array}\right)}_{\boldsymbol{B}_{T}^{*}} \underbrace{\left(\begin{array}{c}
X_{t-1, T} \\
X_{t-2, T} \\
\vdots \\
X_{t-p, T}
\end{array}\right)}_{\boldsymbol{X}_{t-1, T}^{*}}+\underbrace{\left(\begin{array}{c}
\varepsilon_{t} \\
O_{H} \\
\vdots \\
O_{H}
\end{array}\right)}_{\varepsilon_{t}^{*}} .
$$

Here, $\boldsymbol{X}_{t, T}^{*}$ is a $p$-dimensional vector of functions taking values in $H_{\mathbb{R}}^{p}$. Together with the inner product $\langle x, y\rangle=\sum_{i=1}^{p}\left\langle x_{i}, y_{i}\right\rangle$ it forms a Hilbert space. The $\boldsymbol{B}_{u}^{*}$ denotes a matrix of operators and we can write the functional $\operatorname{AR}(p)$ therefore more compactly as

$$
\boldsymbol{X}_{t, T}^{*}=\boldsymbol{B}_{\frac{t}{T}}^{*}\left(\boldsymbol{X}_{t-1, T}^{*}\right)+\varepsilon_{t}^{*}
$$

with $\varepsilon_{t}^{*} \in L^{2}\left(\Omega, H_{\mathbb{R}}^{p}, \mathbb{P}\right)$. Before proving the theorem, we state a lemma that is required in the proof.

Lemma 3.4.2. For $u \in[0,1]$, the assumption $\sum_{j=1}^{p}\left\|B_{u, j}\right\|_{\infty}<1$ implies that the operator $\boldsymbol{B}_{u}^{*}$ satisfies $\left\|\boldsymbol{B}_{u}^{* k_{o}}\right\|_{\infty}<1$ for some $k_{o} \geqslant 1 \in \mathbb{Z}$.

Proof of Lemma 3.4.2. We follow the lines of Bosq (2000)[Theorem 5.2, Corollary 5.1]. To ease notation, we shall write $I$ and $O$ for the identity and zero operator on $H$, respectively while we denote the identity operator on $H^{p}$ by $I_{H^{p}}$. Consider the bounded linear operator $P(\lambda)_{u}$ on $H$

$$
P(\lambda)_{u}=\lambda_{u}^{p} I-\lambda_{u}^{p-1} B_{u, 1}-\ldots-\lambda_{u} B_{u, p-1}-B_{u, p}, \quad \lambda_{u} \in \mathbb{C} .
$$

It is straightforward to derive that, under the assumption $\sum_{j=1}^{p}\left\|B_{u, j}\right\|_{\infty}<1$, noninvertibility of $P(\lambda)_{u}$ implies that $\lambda_{u}$ has modulus strictly less than 1 . Define the following invertible matrices on the complex extension $H_{\mathbb{R}}^{p}$

$$
U\left(\lambda_{u}\right)=\left(\begin{array}{ccccc}
I & \lambda_{u} I & \lambda_{u}^{2} I & \cdots & \lambda_{u}^{p-1} I \\
O & I & \lambda_{u} I & \cdots & \lambda_{u}^{p-2} I \\
\vdots & \ddots & & \vdots & \\
\vdots & & & I & \lambda_{u} I \\
O & \ldots & \ldots & O & I
\end{array}\right)
$$

and

$$
M\left(\lambda_{u}\right)=\left(\begin{array}{ccccc}
O & -I & O & \ldots & O \\
O & O & -I & \ldots & O \\
\vdots & \ddots & & \vdots & \\
O & \ldots & \ldots & O & -I \\
P_{u, 0}(\lambda) & P_{u, 1}(\lambda) & \ldots & \ldots & P_{u, p-1}(\lambda)
\end{array}\right)
$$


where $P_{u, 0}(\lambda)=O$ and $P_{u, j}(\lambda)=\lambda_{u} P_{u, j-1}(\lambda)-B_{u, j}$ for $j=1, \ldots, p$. Then

$$
M\left(\lambda_{u}\right)\left(\lambda_{u} I_{p}-B_{u}^{*}\right) U\left(\lambda_{u}\right)=\left(\begin{array}{ccccc}
I & O & \ldots & O & O \\
O & I & \ldots & \vdots & \vdots \\
\vdots & & & I & O \\
O & \ldots & \ldots & O & P(\lambda)_{u}
\end{array}\right)
$$

from which it follows that $\left(\lambda_{u} I^{H^{p}}-\boldsymbol{B}_{u}^{*}\right)$ is not invertible when $P(\lambda)_{u}$ is not invertible. In other words, the spectrum $S_{u}$ of $\boldsymbol{B}_{u}^{*}$ over the complex extension of $H^{p}$, which is a closed set, satisfies

$$
\begin{aligned}
S_{u}=\left\{\lambda_{u}: \lambda_{u} I^{H^{p}}-B_{u}^{*} \text { not invertible }\right\} & \subset\left\{\lambda_{u}: P(\lambda)_{u} \text { not invertible }\right\} \\
& =\left\{\lambda_{u}:\left|\lambda_{u}\right|<1\right\} .
\end{aligned}
$$

Hence, the assumption that $\sum_{j=1}^{p}\left\|B_{u, j}\right\|_{\infty}<1$ for all $u$, implies the spectral radius of $\boldsymbol{B}_{u}^{*}$ satisfies

$$
r\left(\boldsymbol{B}_{u}^{*}\right)=\sup _{\lambda(u) \in S_{u}}|\lambda(u)|=\lim _{k \rightarrow \infty}\left\|\boldsymbol{B}_{u}^{* k}\right\|_{\infty}^{1 / k}<\frac{1}{1+\delta}
$$

for some $\delta>0$. The equality is a well-known result for the spectral radius of bounded linear operators ${ }^{1}$ and can for example be found in Dunford and Schwartz (1958). From (3.24) it is now clear that there exists a $k_{0} \in \mathbb{Z}, \alpha \in(0,1)$ and a constant $c_{1}$ such that

$$
\left\|\boldsymbol{B}_{u}^{* k}\right\|_{\infty}<c_{1} \alpha^{k}, k \geqslant k_{0} .
$$

Finally, it has been shown in Bosq (2000)[p.74] that this is equivalent to the condition $\left\|\boldsymbol{B}_{u}^{* k_{0}}\right\|_{\infty}<1$ for some integer $k_{0} \geqslant 1$.

We note that this is a weaker assumption than $\left\|\boldsymbol{B}_{u}^{*}\right\|_{\infty}<1$. Although $\left\|\boldsymbol{B}_{u}^{* k_{0}}\right\|_{\infty}<$ 1 is usually stated as the condition for a causal solution in the stationary case, the condition $\sum_{j=1}^{p}\left\|B_{u, j}\right\|_{\infty}<1$ is easier to check in practice. We will now show that a causal solution exists also in the locally stationary setting.

Proof of Theorem 3.4.1. First observe that by recursive substitution

$$
\boldsymbol{X}_{t, T}^{*}=\sum_{l=0}^{\infty}\left(\prod_{s=0}^{l-1} \boldsymbol{B}_{\frac{t-s}{T}}^{*}\right) \varepsilon_{t-l}^{*}
$$

From (3.23), this implies a solution is given by

$$
X_{t, T}=\sum_{l=0}^{\infty}\left[\prod_{s=0}^{l-1} \boldsymbol{B}_{\frac{t-s}{T}}^{*}\right]_{1,1}\left(\varepsilon_{t-l}\right)
$$

\footnotetext{
${ }^{1}$ Gelfand's formula
} 
where $[\cdot]_{1,1}$ refers to the upper left block element of the corresponding block matrix of operators. In order to prove the theorem we will show in a similar manner as Künsch (1995) that

$$
\sup _{t, T}\left\|\left[\prod_{s=0}^{l-1} \boldsymbol{B}_{\frac{t-s}{T}}^{*}\right]_{1,1}\right\|_{\infty}<c \rho^{l} .
$$

for some constant $c$ and $\rho<1$. The proof requires yet another lemma:

Lemma 3.4.3. Let $B(H)$ be the algebra of bounded linear operators on a Hilbert space. Then for each $A \in B(H)$ and each $\varepsilon>0$, there exists an invertible element $M$ of $B(H)$ such that $r(A) \leqslant\left\|M A M^{-1}\right\|_{\infty} \leqslant r(A)+\varepsilon$.

Since $B(H)$ forms a unital $C^{*}$-algebra, this lemma is a direct consequence of a result in Murphy (1990)[p.74]. From (3.24) and by lemma 3.4.3, we can specify for fixed $u$ a new operator $M(u) \in B(H)$ such that

$$
\left\|M(u) \boldsymbol{B}_{u}^{*} M^{-1}(u)\right\|_{\infty}<\frac{1}{1+\delta / 2} .
$$

Because of the continuity of the autoregressive operators in $u$, we have that for all $u \in[0,1]$, there exists a neighborhood $\mathcal{V}(u)$ such that

$$
\left\|M(u) \boldsymbol{B}_{v}^{*} M^{-1}(u)\right\|_{\infty}<\frac{1}{1+\delta / 3}<1 \quad \text { for } \quad v \in \mathcal{V}(u), \quad u \in[0,1] .
$$

Define now the finite union $\bigcup_{i=1}^{m} \mathcal{V}\left(u_{i}\right)$ with $\mathcal{V}\left(u_{i}\right) \cap \mathcal{V}\left(u_{l}\right)=\varnothing$ for $i \neq l$. Due to compactness and the fact that $\boldsymbol{B}_{u}^{*}=\boldsymbol{B}_{0}^{*}$ for $u \leqslant 0$ this union forms a cover of $(-\infty, 1]$. The preceding then implies that there exists a constant $c$ such that

$$
\left\|\boldsymbol{B}_{v}^{*}\right\|_{\infty} \leqslant C\left\|M\left(u_{i}\right) \boldsymbol{B}_{v}^{*} M^{-1}\left(u_{i}\right)\right\|_{\infty} \quad i=1, . ., m .
$$

Now, fix $t$ and $T$ and define the set $J_{i, l}=\left\{s \geqslant 0: \frac{t-s}{T} \in \mathcal{V}\left(u_{i}\right)\right\} \cap\{0,1, \ldots, l-1\}$. Then specify $\rho=\frac{1}{1+\delta / 3}$ to obtain

$$
\begin{aligned}
\left\|\left(\prod_{s=0}^{l-1} \boldsymbol{B}_{\frac{t-s}{T}}^{*}\right)_{1,1}\right\| \|_{\infty} & \leqslant\left\|\prod_{s=0}^{l-1} \boldsymbol{B}_{\frac{t-s}{T}}^{*}\right\|\left\|_{\infty} \leqslant \prod_{i=1}^{m}\right\| \prod_{s \in J_{i, l}} \boldsymbol{B}_{\frac{t-s}{T}}^{*} \|_{\infty} \\
& \leqslant c^{m} \prod_{i=1}^{m} \prod_{s \in J_{i, l}}\left\|\mid M\left(u_{i}\right) \boldsymbol{B}_{\frac{t-s}{T}}^{*} M^{-1}\left(u_{i}\right)\right\|_{\infty} \\
& \leqslant c^{m} \prod_{i=1}^{m} \rho^{\left|J_{i, l}\right|}=c^{m} \rho^{l},
\end{aligned}
$$

which gives the result.

Theorem 3.4.1 will be used to show that time-varying functional ARMA models for which a functional spectral representation exists, fall under Proposition 3.2.4. In order to do so, we first show that for time-varying functional autoregressive processes there exists a common continuous transfer operator $\mathcal{A}_{u, \omega}$ that satisfies condition (ii) of Proposition 3.2.4. This is then extended to general time-varying functional ARMA models. 
Theorem 3.4.4. Let $\left\{\varepsilon_{t}\right\}_{t \in \mathbb{Z}}$ be a white noise process in $H_{\mathbb{R}}$ and consider the functional autoregressive process given by

$$
\sum_{j=0}^{p} B_{\frac{t}{T}, j}\left(X_{t-j, T}\right)(\tau)=C_{\frac{t}{T}}\left(\varepsilon_{t}(\tau),\right.
$$

where the operators satisfy $B_{u, j}=B_{j, 0}$ and $C_{(u)}=C_{(0)}$ if $u<0$, and $B_{u, j}=B_{j, 1}$ and $C_{(u)}=C_{1}$ if $u>1$. If the process satisfies, for all $u \in[0,1]$ and $l=2$ or $l=\infty$, the conditions

(i) $C_{u}$ is an invertible element of $S_{\infty}(H)$;

(ii) $B_{u, j} \in S_{l}(H)$ for $j=1, \ldots, p$ with $\sum_{j=1}^{p}\left\|B_{u, j}\right\|_{l}<1$ and $B_{0, u}=I_{H}$;

(iii) the mappings $u \mapsto B_{u, j}$ for $j=1, . ., p$ and $u \mapsto C_{u}$, are continuous in $u \in[0,1]$ and differentiable on $u \in(0,1)$ with bounded derivatives,

then Proposition 3.2.4 holds in Schatten l-class norm with

$$
\mathcal{A}_{\frac{t}{T}, \omega}^{(T)}=\frac{1}{\sqrt{2 \pi}}\left(\sum_{j=0}^{p} e^{-\mathrm{i} \omega j} B_{\frac{t}{T}, j}\right)^{-1} C_{\frac{t}{T}} .
$$

As shown in Theorem 3.4.1, a sufficient condition for the difference equation (3.27) to have a causal solution is $\sum_{j=1}^{p}\left\|B_{u, j}\right\|_{\infty}<1$ or $\left\|\boldsymbol{B}_{u}^{* k_{0}}\right\|_{\infty}<1$ for some $k_{0} \geqslant 1$. The moving average operators will then satisfy $\sum_{l=0}^{\infty}\left\|A_{t, l}^{(T)}\right\|_{\infty}<\infty$ and Proposition 3.2.5 shows that $X_{t, T}$ satisfies Proposition 3.2 .4 with $\mathcal{A}_{t, \omega}^{(T)} \in \mathcal{B}_{\infty}$. It can be derived from (3.26) that time-varying functional $\operatorname{AR}(p)$ with causal solution of which the moving average operators satisfy $\sum_{l=0}^{\infty}\|\| \mathcal{A}_{t, l}^{(T)} \|_{2}<\infty$ do not exist. We would need at least $\mathcal{A}_{t, 0}^{(T)}$ to be an invertible element of $S_{\infty}(H)$ and $\sum_{j=1}^{p}\left\|B_{u, j}\right\|_{2}<1$. By Proposition A.1.6, this case is covered by Proposition 3.2.4 with $\mathcal{A}_{\frac{t}{T}, \omega} \in S_{2}(H)$. For stationary functional $\operatorname{AR}(p)$ this is straightforward to verify using backshift operator notation and by solving for the inverse of the autoregressive lag operator. As mentioned in Section 3.3, for operators of the form $\mathcal{A}_{t, \omega}^{(T)} \in \mathcal{B}_{2}$ uniform convergence results can be obtained under slightly more restrictive assumptions. We will come back to this in more detail in Section 3.5 where we consider estimation of the timevarying spectral density operator.

Proof of Theorem 3.4.4. The moving average representation (3.22) and the difference equation (3.27) together imply that the process can be represented as

$$
X_{t, T}(\tau)=\sum_{l=0}^{\infty} A_{t, l}^{(T)}\left(C_{\frac{t-l}{T}}^{-1} \sum_{j=0}^{p} B_{\frac{t-l}{T}, j}\left(X_{t-l-j, T}\right)\right)(\tau) .
$$

Using the linearity of the operators and applying a change of variables $l^{\prime}=l+j$, this can be written as

$$
X_{t, T}(\tau)=\sum_{l^{\prime}=0}^{\infty} \sum_{j=0}^{p} A_{t, l^{\prime}-j}^{(T)} C_{\frac{t-l^{\prime}+j}{T}}^{-1} B_{\frac{t-l^{\prime}+j}{T}, j}\left(X_{t-l^{\prime}, T}\right),
$$


where $A_{t, l^{\prime}-j}^{(T)}=O_{H}$ for $l^{\prime}<j$. For a purely nondeterministic solution we require

$$
\sum_{j=0}^{p} A_{t, l^{\prime}-j}^{(T)} C_{\frac{t-l^{\prime}+j}{T}}^{-1} B_{\frac{t-l^{\prime}+j}{T}, j}= \begin{cases}I_{H} & \text { if } l^{\prime}=0 \\ O_{H} & \text { if } l^{\prime} \neq 0 .\end{cases}
$$

Because $\varepsilon_{t}$ is white noise in $L^{2}([0,1], \mathbb{R})$, it has spectral representation

$$
\varepsilon_{t}=(2 \pi)^{-1 / 2} \int_{-\pi}^{\pi} e^{\mathrm{i} \omega t} d Z_{\omega}, \quad t \in \mathbb{Z} .
$$

Since a solution of the form (3.22) exists, we also have

$$
X_{t, T}=\int_{-\pi}^{\pi} e^{i \omega t} \mathcal{A}_{t, \omega}^{(T)} d Z_{\omega}
$$

where $\mathcal{A}_{t, \omega}^{(T)}=\frac{1}{\sqrt{2 \pi}} \sum_{l=0}^{\infty} A_{t, l}^{(T)} e^{-\mathrm{i} \omega l}$. Substituting the spectral representations of $X_{t, T}$ and $\varepsilon_{t}$ into (3.27), we get together with the linearity of the operators $B_{u, j}$ and $\mathcal{A}_{t, \omega}^{(T)}$

$$
\int_{-\pi}^{\pi} \sum_{j=0}^{p} e^{\mathrm{i} \omega(t-j)} B_{\frac{t}{T}, j} \mathcal{A}_{t-j, \omega}^{(T)} d Z_{\omega}=(2 \pi)^{-1 / 2} \int_{-\pi}^{\pi} e^{\mathrm{i} \omega t} C_{\frac{t}{T}} d Z_{\omega}
$$

Given the operator $\mathcal{A}_{\frac{t}{T}, \omega}$ satisfies equation (3.28), the previous implies we can write

$$
\begin{aligned}
\frac{1}{\sqrt{2 \pi}} C_{\frac{t}{T}} & =\sum_{j=0}^{p} e^{-\mathrm{i} \omega j} B_{\frac{t}{T}, j} \mathcal{A}_{\frac{t}{T}, \omega} \\
& =\sum_{j=0}^{p} e^{-\mathrm{i} \omega j} B_{\frac{t}{T}, j} \mathcal{A}_{\frac{t-j}{T}, \omega}+\sum_{j=0}^{p} e^{-\mathrm{i} \omega j} B_{\frac{t}{T}, j}\left(\mathcal{A}_{\frac{t}{T}, \omega}-\mathcal{A}_{\frac{t-j}{T}, \omega}\right) .
\end{aligned}
$$

From the last equation, it follows that

$$
\begin{aligned}
\sum_{j=0}^{p} e^{\mathrm{i} \omega(t-j)} B_{\frac{t}{T}, j}\left(\mathcal{A}_{t-j, \omega}^{(T)}-\mathcal{A}_{\frac{t-j}{T}, \omega}\right) & =\sum_{j=0}^{p} e^{\mathrm{i} \omega(t-j)} B_{\frac{t}{T}, j}\left(\mathcal{A}_{\frac{t}{T}, \omega}-\mathcal{A}_{\frac{t-j}{T}, \omega}\right) \\
& =C_{\frac{t}{T}} \Omega_{t, \omega}^{(T)}
\end{aligned}
$$

where $\Omega_{t, \omega}^{(T)}=O_{H}, t \leqslant 0$. We will show that this operator is of order $O\left(\frac{1}{T}\right)$ in $S_{l}(H)$. Throughout the rest of the proof, we focus on the case $l=2$. By Proposition A.1.6, the smooth transfer operator satisfies $\mathcal{A}_{u, \omega} \in S_{2}(H)$. Under the conditions of Theorem 3.4.4, we have that for any element $\psi \in L^{2}([0,1], \mathbb{C})$ and fixed $\omega \in$ $\Pi$, the mapping $u \mapsto \mathcal{A}_{u, \omega}(\psi)(\tau)$ is continuous and, from the properties of the $B_{u, j}$, is differentiable and has bounded derivatives with respect to $u$. Therefore $\mathcal{A}_{u, \omega}(\psi)(\tau)=\left\langle a_{u, \omega}, \psi\right\rangle$, which implies by continuity of the inner product that the kernels $a_{u, \omega} \in L^{2}\left([0,1]^{2}, \mathbb{C}\right)$ are Lipschitz continuous with respect to $u$. Letting $K$ denote the Lipschitz constant, we have

$$
\sup _{t, \omega}\left\|a_{\frac{t}{T}, \omega}-a_{\frac{t-j}{T}, \omega}\right\|_{2} \leqslant K\left|\frac{j}{T}\right|
$$


for all $\omega \in \Pi$, uniformly in $u$. Hence,

$$
\sup _{t, \omega}\left\|\mathcal{A}_{\frac{t}{T}, \omega}-\mathcal{A}_{\frac{t-j}{T}, \omega}\right\|_{2}=\sup _{t, \omega}\left\|a_{u, \frac{t}{T}} \omega-a_{u, \frac{t-j}{T}} \omega\right\|_{2}=O\left(\frac{1}{T}\right),
$$

and it easily follows from (3.31) and Proposition A.1.3 that $\left\|C_{\frac{t}{T}} \Omega_{t, \omega}^{(T)}\right\|_{2}=O\left(\frac{1}{T}\right)$ uniformly in $t, \omega$. From (3.29), we additionally have

$$
\begin{aligned}
\sum_{l=0}^{t} A_{t, l}^{(T)} \Omega_{t-l, \omega}^{(T)} & =\sum_{l=0}^{t} \sum_{j=0}^{p} A_{t, l-j}^{(T)} C_{\frac{t-l+j}{T}}^{-1} B_{\frac{t-l+j}{T}, j} e^{\mathrm{i} \omega(t-l)}\left[\mathcal{A}_{t-l, \omega}^{(T)}-\mathcal{A}_{\frac{t-l}{T}, \omega}\right] \\
& =e^{\mathrm{i} \omega t}\left[\mathcal{A}_{t, \omega}^{(T)}-\mathcal{A}_{\frac{t}{T}, \omega}\right] .
\end{aligned}
$$

Since the moving average operators are either in $S_{2}(H)$ or in $S_{\infty}(H)$, the above together with another application of Hölder's Inequality for operators yields

$$
\sup _{t, \omega}\left\|\mathcal{A}_{t, \omega}^{(T)}-\mathcal{A}_{\frac{t}{T}, \omega}\right\|_{2} \leqslant \sup _{t, \omega}\left(\left\|A_{t, 0}^{(T)}\right\|\left\|_{\infty}\right\| \Omega_{t, \omega}^{(T)}\left\|_{2}+\sum_{l=1}^{t}\right\|\left\|A_{t, l}^{(T)}\right\|\left\|_{2}\right\| \Omega_{t-l, \omega}^{(T)} \|_{2}\right) \leqslant \frac{K}{T},
$$

for some constant $K$ independent of $T$.

Remark 3.4.5 (Case $l=\infty$ ). In case it is only assumed that the moving average operators are summable in operator norm and $\sum_{j=1}^{p}\left\|B_{\frac{t}{T}, j}\right\|_{\infty}<1$ (or the weaker assumption in (3.25)), condition (ii) of Proposition 3.2.4 does not hold in HilbertSchmidt norm. Rather, the condition only holds in operator norm. In this case, Theorem A.1.8 yields

$$
\sup _{t, \omega}\left\|\mathcal{A}_{\frac{t}{T}, \omega}-\mathcal{A}_{\frac{t-j}{T}, \omega}\right\|_{\infty} \leqslant\left|\frac{j}{T}\right|\left\|\frac{\partial}{\partial u} A_{u, \omega}\right\|_{\infty}=O\left(\frac{1}{T}\right),
$$

and by the equality in (3.31), we find $\left\|C_{\frac{t}{T}} \Omega_{t, \omega}^{(T)}\right\|_{\infty}=O\left(\frac{1}{T}\right)$ uniformly in $t, \omega$.

It can now easily be shown that the time-varying functional ARMA processes are locally stationary in the sense of Proposition 3.2.4. A time-varying functional moving average process of order $q$ will have transfer operator

$$
\mathcal{A}_{t, \omega}^{(T)}=\frac{1}{\sqrt{2 \pi}} \sum_{j=0}^{q} \Phi_{\frac{t}{T}, j} e^{-\mathrm{i} \omega j}
$$

where $\Phi_{t / T, j} \in S_{l}(H)$ are the moving average filter operators. This follows from the spectral representation of the $\varepsilon_{t}$ as given in (3.30). Taking $\mathcal{A}_{\frac{t}{T}, \omega}=\mathcal{A}_{t, \omega}^{(T)}$ gives the result. Finally, we can combine this with the above theorem to obtain that Proposition 3.2.4 holds for time-varying functional $\operatorname{ARMA}(p, q)$ with common continuous transfer operator given by

$$
\mathcal{A}_{\frac{t}{T}, \omega}=\frac{1}{\sqrt{2 \pi}} C_{\frac{t}{T}}\left(\sum_{j=0}^{p} e^{-\mathrm{i} \omega j} B_{\frac{t}{T}, j}\right)^{-1} \sum_{l=0}^{q} \Phi_{\frac{t}{T}, l} e^{-\mathrm{i} \omega l} .
$$


Remark 3.4.6 (Cramér representation stationary functional ARMA). It is straightforward to see that when the operators do not depend on $t$ and $T$ we obtain stationary functional $\operatorname{ARMA}(p, q)$ processes as discussed in Bosq (2000) or, as explained in Remark 3.2.6, the model introduced in Hörmann and Kokoszka (2010). We therefore showed that these models also have a well-defined functional Cramér representation.

\subsection{Estimation}

The time-varying spectral density operator as defined in section 3.3.2 allows to capture the second-order structure of a functional time series with possibly changing dynamics. In order to consider inferential techniques such as dynamic functional principal components for time-varying functional time series, we require a way to consistently estimate the time-varying spectral density operator. In this section, we present a nonparametric estimator of the time-varying spectral density operator. First, a functional version of the segmented periodogram operator and its kernel are introduced and the mean and covariance structure are obtained. We will then consider a smoothed version of this operator and show it is consistent. Furthermore, a central limit theorem is derived at the end of this section. For this, we need the specify also the structure of our functional orthogonal increment process $Z_{\omega}$ as given in Proposition 3.2.4. Using Theorem 3.2.2, we can assume its dependence structure satisfies:

Assumption 3.5.1. For fixed $\omega, Z_{\omega}$ is a random element of $L^{2}([0,1], \mathbb{C})$ with $E\left\|Z_{\omega}\right\|_{2}^{2}=\int_{-\pi}^{\omega}\left\|\mathcal{F}_{\lambda}^{\varepsilon}\right\|_{1} d \lambda$, and the process $\omega \mapsto Z_{\omega}$ satisfies

$$
\begin{aligned}
\mathbb{E}\left\langle Z_{\omega_{1}}-Z_{\omega_{2}}, Z_{\omega_{3}}-Z_{\omega_{4}}\right\rangle & =0 \text { for } \omega_{1}>\omega_{2} \geqslant \omega_{3}>\omega_{4}, \\
\operatorname{cum}\left(Z_{\omega_{1}}\left(\tau_{1}\right), \ldots, Z_{\omega_{k}}\left(\tau_{k}\right)\right)= & \int_{-\pi}^{\omega_{1}} \cdots \int_{-\pi}^{\omega_{k}} \eta\left(\lambda_{1}+. .+\lambda_{k}\right) \\
& \times f_{\lambda_{1}, . ., \lambda_{k-1}}^{\varepsilon}\left(\tau_{1}, . ., \tau_{k}\right) d \lambda_{1} d \lambda_{2} \ldots d \lambda_{k},
\end{aligned}
$$

where $f_{\omega_{0}}^{\varepsilon}(\tau)=\mathbb{E} \varepsilon_{0}(\tau)=0$ and for $k \geqslant 2, \sup _{\omega_{1}, \ldots, \omega_{k-1}}\left\|f_{\omega_{1}, . ., \omega_{k-1}}^{\varepsilon}\right\|_{p} \leqslant\|\mathcal{k}\|_{p}<\infty$ with $\kappa\left(\tau_{1}, . ., \tau_{k}\right):[0,1]^{k} \rightarrow \mathbb{R}$ and $p=2$ or $p=\infty$.

\subsubsection{The functional segmented periodogram}

The general idea underlying inference methods in the setting of locally stationary processes is that the process $X_{t, T}$ can be considered to be close to some stationary process, say $X_{t}^{\left(u_{0}\right)}$, on a reasonably small data-segment around $u_{0}$. If this segment is described by $\left\{t:\left|\frac{t}{T}-u_{0}\right| \leqslant b_{\mathrm{t}} / 2\right\}$ for some bandwidth $b_{\mathrm{t}}$, classical estimation methods from the stationary framework can be applied on this stretch. The estimated value is subsequently assigned to be the value of the parameter curve at the midpoint $u_{o}$ of the segment. The entire parameter curve of interest in time direction can then be obtained by shifting the segment. We will also apply this technique in the functional setting. 
First, let the length of the stretch considered for estimation be denoted by $N_{T}$, where $N_{T}$ is even and $N_{T} \ll T$. In the following, we will drop the explicit dependence of $N$ on $T$ and simple write $N=N_{T}$. Then the local version of the functional Discrete Fourier Transform (fDFT) is defined as

$$
D_{u, \omega}^{(T)}(\tau)=\sum_{s=0}^{N-1} h_{s, N} X_{\lfloor u T\rfloor-N / 2+s+1, T}(\tau) e^{-\mathrm{i} \omega s},
$$

where $h_{s, N}$ is a data taper of length $N$. It is clear that $D_{u, \omega}^{(T)}$ is a $2 \pi$-periodic function in $\omega$ that takes values in $H$. The data taper is used to improve the finite sample properties of the estimator (Dahlhaus, 1988): firstly, it mitigates spectral leakage, which is the transfer of frequency content from large peaks to surrounding areas and is also a problem in the stationary setting. Secondly, it reduces the bias that stems from the degree of nonstationarity of the process on the given data-segment, that is, the fact that we use the observations $X_{t, T}$ for estimation rather than the unknown stationary process $X_{t}^{\left(u_{0}\right)}$. We define the data taper by a function $h:[0,1] \rightarrow \mathbb{R}$ and setting $h_{s, N}=h\left(\frac{s}{N}\right)$; the taper function $h$ should decay smoothly to zero at the endpoints of the interval while being essentially equal to 1 in the central part of the interval. Thus the taper gives more weight to data points closer to the midpoint. More particularly, we impose the following conditions of the taper function $h$.

Assumption 3.5.2 (Taper function). The taper function $h: \mathbb{R} \rightarrow \mathbb{R}^{+}$is symmetric with compact support on $[0,1]$ and is of bounded variation.

As a basis for estimation of the time-varying spectral density operator, we consider the normalized tensor product of the local functional Discrete Fourier Transform. This leads to the concept of a segmented or localized periodogram tensor

$$
I_{u, \omega}^{(T)}=\left(2 \pi H_{2, N}(0)\right)^{-1} D_{u, \omega}^{(T)} \otimes D_{u,-\omega}^{(T)},
$$

where

$$
H_{k, N}(\omega)=\sum_{s=0}^{N-1} h_{s, N}^{k} e^{-\mathrm{i} \omega s}
$$

is the finite Fourier transform of the $k$-th power of the data taper. Given the moments are well-defined in $L^{2}\left([0,1]^{2}, \mathbb{C}\right)$, the operator $(3.35)$ is induced by the localized periodogram kernel

$$
I_{u, \omega}^{(T)}(\tau, \sigma)=\left(2 \pi H_{2, N}(0)\right)^{-1} D_{u, \omega}^{(T)}(\tau) D_{u,-\omega}^{(T)}(\sigma) .
$$

We remark that, similar to the stationary case, sufficient conditions for the existence of the higher order moments of the localized periodogram tensor are obtained from

$$
\left\|I_{u, \omega}^{(T)}\right\|_{2}^{\rho}=\left(2 \pi H_{2, N}(0)\right)^{-\rho}\left\|D_{u, \omega}^{(T)}\right\|_{2}^{2 \rho}
$$

which implies that $\mathbb{E}\left\|I_{u, \omega}^{(T)}\right\|_{2}^{\rho}<\infty$ if $\mathbb{E}\left\|D_{u, \omega}^{(T)}\right\|_{2}^{2 \rho}<\infty$ or, in terms of moments of $X$, $\mathbb{E}\left\|X_{t, T}\right\|_{2}^{2 \rho}<\infty$.

To ease notation, we denote $t_{u, r}=\lfloor u T\rfloor-N / 2+r+1$ to be the $r$-th element of the data-segment with midpoint $u$. For $u_{j}=j / T$ we also write $t_{j, r}=t_{u_{j}, r}$ and abbreviate $u_{j, r}=t_{j, r} / T$. The following result is used throughout the rest of the chapter. 
Proposition 3.5.3. Let the conditions of Proposition 3.2.4 be satisfied with $\mathcal{A}_{t, \omega}^{(T)} \in$ $\mathcal{B}_{\infty}$ and $\sup _{\omega_{1}, \ldots, \omega_{k-1}}\left\|\mathcal{F}_{\omega_{1}, \ldots, \omega_{k-1}}^{\varepsilon}\right\|_{2}<\infty$. Then

$$
\begin{aligned}
\operatorname{cum}\left(X_{t_{r_{1}}, T}, \ldots, X_{t_{r_{k}}, T}\right)= & \int_{\Pi^{k}} e^{\mathrm{i}\left(\lambda_{1} r_{1}+\ldots+\lambda_{k} r_{k}\right)}\left(\mathcal{A}_{t_{r_{1}}, \lambda_{1}}^{(T)} \otimes \cdots \otimes \mathcal{A}_{t_{r_{k}}, \lambda_{k}}^{(T)}\right) \\
& \times \eta\left(\lambda_{1}+\ldots+\lambda_{k}\right) \mathcal{F}_{\lambda_{1}, \ldots, \lambda_{k-1}}^{\varepsilon} d \lambda_{1} \cdots d \lambda_{k},
\end{aligned}
$$

where the equality holds in the tensor product space $H \otimes \cdots \otimes H$. Moreover, for fixed $t \in\{1, \ldots, T\}$ and $T \in \mathbb{N}$, the $k$-th order cumulant spectral tensor of the linear functional process $\left\{X_{t, T}\right\}$,

$$
\mathcal{F}_{\lambda_{1}, \ldots, \lambda_{k-1}}^{(t, T)}=\left(\mathcal{A}_{t_{r_{1}}, \lambda_{1}}^{(T)} \otimes \cdots \otimes \mathcal{A}_{t_{r_{k-1}}, \alpha_{k-1}}^{(T)} \otimes \mathcal{A}_{t_{r_{k}},-\lambda_{+}}^{(T)}\right) \mathcal{F}_{\lambda_{1}, \ldots, \lambda_{k-1}}^{\varepsilon},
$$

where $\lambda_{+}=\lambda_{1}+\ldots+\lambda_{k-1}$ of the linear functional process $\left\{X_{t, T}\right\}$ is well-defined in the tensor product space $\bigotimes_{i=1}^{k} H$ with kernel $f_{\lambda_{1}, \ldots, \lambda_{k-1}}^{(t, T)}\left(\tau_{1}, \ldots, \tau_{k}\right)$. For $k=1$ the corresponding operator $\mathcal{F}_{\omega}^{(t, T)}$ is an element of $S_{1}(H)$.

The proof can be found in Section A.4.3 of the appendix. We remark that under the stronger condition $\mathcal{A}_{t, \omega}^{(T)} \in \mathcal{B}_{2}$, the tensor $\mathcal{F}_{\lambda_{1}, . ., \lambda_{k-1}}^{(t, T)}$ will be Trace-class for all $k \geqslant 2$. The above proposition implies that the higher order cumulant tensor of the local fDFT can be written as

$$
\begin{aligned}
\operatorname{cum}\left(D_{u, \omega_{1}}^{(T)}, \ldots, D_{u, \omega_{k}}^{(T)}\right) & \\
= & \int_{\Pi^{k}}\left(H_{N}\left(\mathcal{A}_{t_{u, \bullet}, \lambda_{1}}^{(T)}, \omega_{1}-\lambda_{1}\right) \otimes \cdots \otimes H_{N}\left(\mathcal{A}_{t_{u, \bullet}, \lambda_{k}}^{(T)}, \omega_{k}-\lambda_{k}\right)\right) \\
& \quad \times \eta\left(\lambda_{1}+\ldots+\lambda_{k}\right) \mathcal{F}_{\lambda_{1}, \ldots, \lambda_{k-1}}^{\varepsilon} d \lambda_{1} \cdots d \lambda_{k} .
\end{aligned}
$$

Here, the function $H_{N}\left(G_{\bullet}, \omega\right)$ and similarly $H_{k, N}\left(G_{\bullet}, \omega\right)$ generalize the definitions of $H_{N}$ and $H_{k, N}$ to

$$
H_{k, N}\left(G_{\bullet}, \omega\right)=\sum_{s=0}^{N-1} h_{s, N}^{k} G_{s} e^{-\mathrm{i} \omega s}
$$

with $H_{N}\left(G_{\bullet}, \omega\right)=H_{1, N}\left(G_{\bullet}, \omega\right)$, where in our setting $G_{s} \in \mathcal{B}_{\infty}$ for all $s \in \mathbb{N}_{0}$. For $G_{\bullet}=I_{H}$, we get back the original definitions of $H_{N}$ and $H_{k, N}$. The convolution property of $H_{N}$ straightforwardly generalizes to

$$
\int_{-\pi}^{\pi} H_{k, N}\left(A_{\bullet}, \alpha+\gamma\right) \otimes H_{l, N}\left(B_{\bullet}, \beta-\gamma\right) d \gamma=2 \pi H_{k+l, N}\left(A_{\bullet} \otimes B_{\bullet}, \alpha+\beta\right),
$$

where $\left(A_{r}\right)_{r=0, \ldots, N-1}$ and $\left(B_{r}\right)_{r=0, \ldots, N-1}$ are vectors of tensors or operators.

From the taper function $h$, we derive the smoothing kernel $K_{\mathrm{t}}$ in rescaled time $u$ by

$$
K_{\mathrm{t}}(x)=\frac{1}{H_{2}} h\left(x+\frac{1}{2}\right)^{2}
$$

for $x \in\left[-\frac{1}{2}, \frac{1}{2}\right]$ and zero elsewhere; furthermore, we define the bandwidth $b_{\mathrm{t}, T}=N / T$ that corresponds to segments of length $N$, and set $K_{\mathrm{t}, T}(x)=\frac{1}{b_{\mathrm{t}, T}} K_{\mathrm{t}}\left(\frac{x}{b_{\mathrm{t}, T}}\right)$. Finally, we define the kernel-specific constants

$$
\kappa_{\mathrm{t}}=\int_{\mathbb{R}} x^{2} K_{\mathrm{t}}(x) d x \quad \text { and } \quad\left\|K_{\mathrm{t}}\right\|_{2}^{2}=\int_{\mathbb{R}} K_{\mathrm{t}}(x)^{2} d x
$$


The first order and second order properties of the segmented functional periodogram can now be determined.

Theorem 3.5.4. Let $\left\{X_{t, T}\right\}$ be a locally stationary process in $H_{\mathbb{R}}$ satisfying Proposition 3.2.4 with $\mathcal{A}_{t, \omega}^{(T)} \in \mathcal{B}_{\infty}$ and $\sup _{\omega_{1}, \ldots, \omega_{k-1}}\left\|\mathcal{F}_{\omega_{1}, \ldots, \omega_{k-1}}^{\varepsilon}\right\|_{2}<\infty$ for $k=2$, 4. Additionally, let the operator-valued function $\mathcal{A}_{u, \omega}$ be Hölder continuous of order $\alpha>1 / 2$ and twice continuously differentiable in both $u$ and $\omega$. Then the mean and covariance structure of the local functional periodogram are given by

$$
\mathbb{E} I_{u, \omega}^{(T)}(\tau, \sigma)=f_{u, \omega}(\tau, \sigma)+\frac{1}{2} \kappa_{\mathrm{t}} b_{\mathrm{t}, T}^{2} \frac{\partial^{2} f_{u, \omega}(\tau, \sigma)}{\partial u^{2}}+o\left(b_{\mathrm{t}, T}^{2}\right)+O\left(\frac{\log \left(b_{\mathrm{f}, T} T\right)}{b_{\mathrm{f}, T} T}\right),
$$

and

$$
\begin{aligned}
& \operatorname{cov}\left(I_{u, \omega_{1}}^{(T)}\left(\tau_{1}, \sigma_{1}\right), I_{u, \omega_{2}}^{(T)}\left(\tau_{2}, \sigma_{2}\right)\right) \\
& =H_{2, N}\left(f_{\frac{t_{u, \bullet}, \omega_{1}}{T}}\left(\tau_{1}, \tau_{2}\right), \omega_{1}-\omega_{2}\right) H_{2, N}\left(f_{\frac{t_{u, \bullet}}{T},-\omega_{1}}\left(\sigma_{1}, \sigma_{2}\right), \omega_{2}-\omega_{1}\right) \\
& +H_{2, N}\left(f_{\frac{t_{u, \bullet}, \omega_{1}}{T}}\left(\tau_{1}, \sigma_{2}\right), \omega_{1}+\omega_{2}\right) H_{2, N}\left(f_{\frac{t_{u, \bullet},-\omega_{1}}{T}}\left(\sigma_{1}, \tau_{2}\right),-\omega_{1}-\omega_{2}\right) \\
& +O\left(\frac{\log (N)}{N}\right)+O\left(\frac{1}{N}\right)
\end{aligned}
$$

in $L^{2}$.

The proof can be found in section A.4.3. In particular, it exploits condition ( $i$ i of Proposition 3.2.4 and uses the theory of $L$-functions (Dahlhaus, 1983) to provide upper bound conditions on the data taper function. For details of the latter we additionally refer to Section A.3 of the appendix.

\subsubsection{Consistent estimation}

Theorem 3.5.4 shows that the functional segmented periodogram is not a consistent estimator. In order to obtain a consistent estimator we proceed by smoothing the raw estimator over different frequencies. That is, we consider convolving the segmented periodogram kernel with a window function in frequency direction

$$
\hat{f}_{u, \omega}^{(T)}(\tau, \sigma)=\frac{1}{b_{\mathrm{f}, T}} \int_{\pi}^{\pi} K_{\mathrm{f}}\left(\frac{\omega-\lambda}{b_{\mathrm{f}, T}}\right) I_{u, \lambda}^{(T)}(\tau, \sigma) d \lambda,
$$

where $b_{\mathrm{f}, T}$ denotes the bandwidth in frequency direction. We make the following assumption about the kernel function.

Assumption 3.5.5 (Kernel function). The frequency kernel function $K_{\mathrm{f}}: \mathbb{R} \rightarrow \mathbb{R}^{+}$ is symmetric, has bounded variation and compact support $[-1,1]$, and satisfies
(i) $\int_{\mathbb{R}} K_{\mathrm{f}}(\omega) d \omega=1$;
(ii) $\int_{\mathbb{R}} \omega K_{\mathrm{f}}(\omega) d \omega=0$. 
To ease notation, we also write $K_{\mathrm{f}, T}(\omega)=\frac{1}{b_{\mathrm{f}, T}} K_{\mathrm{f}}\left(\frac{\omega}{b_{\mathrm{f}, T}}\right)$. Additionally we use subsequently

$$
\kappa_{\mathrm{f}}=\int_{\mathbb{R}} \omega^{2} K_{\mathrm{f}}(\omega) d \omega \quad \text { and } \quad\left\|K_{\mathrm{f}}\right\|_{2}^{2}=\int_{\mathbb{R}} K_{\mathrm{f}}^{2}(\omega) d \omega
$$

as an abbreviation for kernel--specific constants.

Theorem 3.5.6 (Properties of the spectral density estimator). Let $\left\{X_{t, T}\right\}$ be a locally stationary process in $H_{\mathbb{R}}$ satisying Proposition 3.2.4 with $\mathcal{A}_{t, \omega}^{(T)} \in \mathcal{B}_{\infty}$ and $\sup _{\omega_{1}, \ldots, \omega_{k-1}}\left\|\mathcal{F}_{\omega_{1}, \ldots, \omega_{k-1}}^{\varepsilon}\right\|_{2}<\infty$ for $k=2,4$, and let the operator-valued function $\mathcal{A}_{u, \omega}$ be Hölder continuous of order $\alpha>1 / 2$ and twice continuously differentiable in both $u$ and $\omega$. Additionally, the kernel $K_{\mathrm{f}}$ satisfies Assumption 3.5.5. Then the estimator

$$
\hat{f}_{u, \omega}^{(T)}(\tau, \sigma)=\int_{-\pi}^{\pi} K_{\mathrm{f}, T}(\omega-\lambda) I_{u, \lambda}^{(T)}(\tau, \sigma) d \lambda
$$

has mean

$$
\begin{gathered}
\mathbb{E} \hat{f}_{u, \omega}^{(T)}(\tau, \sigma)=f_{u, \omega}(\tau, \sigma)+\frac{1}{2} b_{\mathrm{t}, T}^{2} \kappa_{\mathrm{t}} \frac{\partial^{2} f_{u, \omega}(\tau, \sigma)}{\partial u^{2}}+\frac{1}{2} b_{\mathrm{f}, T}^{2} \kappa_{\mathrm{f}} \frac{\partial^{2} f_{u, \omega}(\tau, \sigma)}{\partial \omega^{2}} \\
+o\left(b_{\mathrm{t}, T}^{2}\right)+o\left(b_{\mathrm{f}, T}^{2}\right)+O\left(\frac{\log \left(b_{\mathrm{t}, T} T\right)}{b_{\mathrm{t}, T} T}\right)
\end{gathered}
$$

and covariance structure

$$
\begin{aligned}
\operatorname{cov}\left(\hat{f}_{u, \omega_{1}}^{(T)}\left(\tau_{1}, \sigma_{1}\right), \hat{f}_{u, \omega_{2}}^{(T)}\left(\tau_{2}, \sigma_{2}\right)\right) & \\
= & \frac{2 \pi\left\|K_{\mathrm{t}}\right\|_{2}^{2}}{b_{\mathrm{t}, T} T} \int_{-\pi}^{\pi} K_{\mathrm{f}, T}\left(\omega_{1}-\lambda_{1}\right) K_{\mathrm{f}, T}\left(\omega_{2}-\lambda_{1}\right) f_{u, \lambda_{1}}\left(\tau_{1}, \tau_{2}\right) f_{u,-\lambda_{1}}\left(\sigma_{1}, \sigma_{2}\right) d \lambda_{1} \\
& +\frac{2 \pi\left\|K_{\mathrm{t}}\right\|_{2}^{2}}{b_{\mathrm{f}, T} T} \int_{-\pi}^{\pi} K_{\mathrm{f}, T}\left(\omega_{1}-\lambda_{1}\right) K_{\mathrm{f}, T}\left(\omega_{2}+\lambda_{1}\right) f_{u, \lambda_{1}}\left(\tau_{1}, \sigma_{2}\right) f_{u,-\lambda_{1}}\left(\sigma_{1}, \tau_{2}\right) d \lambda_{1} \\
& +O\left(\frac{\log \left(b_{\mathrm{t}, T} T\right)}{b_{\mathrm{t}, T} T}\right)+O\left(\frac{b_{\mathrm{t}, T}}{T}\right)+O\left(\left(b_{\mathrm{t}, T} b_{\mathrm{f}, T} T\right)^{-2}\right)
\end{aligned}
$$

in $L^{2}$.

The proof follows from a multivariate Taylor expansion and an application of Lemma P4.1 of Brillinger (1981) and is given in Section A.4.3 of the appendix. We note that the covariance has greatest magnitude for $\omega_{1} \pm \omega_{2} \equiv 0(\bmod 2 \pi)$, where the weight is concentrated in a band of width $O\left(b_{\mathrm{f}, T}\right)$ around $\omega_{1}$ and $\omega_{2}$ respectively. The above theorem demonstrates that, in order for the error terms to disappear, we need the bandwidths to decay at an appropriate rate.

Assumption 3.5.7 (bandwidths). As $T$ tends to infinity, the bandwidths satisfy $b_{\mathrm{f}, T} \rightarrow 0$ and $b_{\mathrm{t}, T} \rightarrow 0$ such that $\left.\left.i\right) b_{\mathrm{f}, T} b_{\mathrm{t}, T} T \rightarrow \infty, i i\right) b_{\mathrm{f}, T} \log \left(b_{\mathrm{t}, T} T\right) \rightarrow 0$ and iii) $b_{\mathrm{t}, T}^{2} b_{\mathrm{f}, T} \rightarrow 0$. 
We then have the following proposition.

Proposition 3.5.8. Assume the conditions of Theorem 3.5.6 hold and that the banwidths $b_{\mathrm{t}}, b_{\mathrm{f}}$ satisfy Assumption 3.5 .7 as $T \rightarrow \infty$. Then

$$
\begin{aligned}
& \lim _{T \rightarrow \infty} b_{\mathrm{t}, T} b_{\mathrm{f}, T} T \operatorname{cov}\left(\hat{f}_{u, \omega_{1}}^{(T)}\left(\tau_{1}, \sigma_{1}\right), \hat{f}_{u, \omega_{2}}^{(T)}\left(\tau_{2}, \sigma_{2}\right)\right) \\
&=2 \pi\left\|K_{\mathrm{t}}\right\|_{2}^{2}\left\|K_{\mathrm{f}}\right\|_{2}^{2} \eta\left(\omega_{1}-\omega_{2}\right) f_{u, \omega_{1}}\left(\tau_{1}, \tau_{2}\right) f_{u,-\omega_{1}}\left(\sigma_{1}, \sigma_{2}\right) \\
& \quad+2 \pi\left\|K_{\mathrm{t}}\right\|_{2}^{2}\left\|K_{\mathrm{f}}\right\|_{2}^{2} \eta\left(\omega_{1}+\omega_{2}\right) f_{u, \omega_{1}}\left(\tau_{1}, \sigma_{2}\right) f_{u,-\omega_{1}}\left(\sigma_{1}, \tau_{2}\right),
\end{aligned}
$$

where the convergence is in $L^{2}$ for fixed $\omega_{1}, \omega_{2}$. If $\omega_{1}, \omega_{2}$ depend on $T$ then convergence in $L^{2}$ holds provided that $\liminf _{T \rightarrow \infty}\left|\left(\omega_{1, T} \pm \omega_{2, T}\right) \bmod 2 \pi\right|>\varepsilon$ for some $\varepsilon>0$.

Proof. A change of variables shows that (3.47) can be written as

$$
\begin{array}{rl}
b_{\mathrm{t}, T} b_{\mathrm{f}, T} & T \operatorname{cov}\left(\hat{f}_{u, \omega_{1}}^{(T)}\left(\tau_{1}, \sigma_{1}\right), \hat{f}_{u, \omega_{2}}^{(T)}\left(\tau_{2}, \sigma_{2}\right)\right) \\
=2 \pi & b_{\mathrm{f}, T}\left\|K_{\mathrm{t}}\right\|_{2}^{2} \int_{-\pi}^{\pi} K_{\mathrm{f}, T}\left(\omega_{1}-\omega_{2}-\lambda\right) K_{\mathrm{f}, T}(\lambda) f_{u, \omega_{2}-\lambda}\left(\tau_{1}, \tau_{2}\right) f_{u,-\omega_{2}-\lambda}\left(\sigma_{1}, \sigma_{2}\right) d \lambda \\
\quad+2 \pi b_{\mathrm{f}, T}\left\|K_{\mathrm{t}}\right\|_{2}^{2} \int_{-\pi}^{\pi} K_{\mathrm{f}, T}\left(\omega_{1}+\omega_{2}-\lambda\right) K_{\mathrm{f}, T}(\lambda) f_{u,-\omega_{2}+\lambda}\left(\tau_{1}, \sigma_{2}\right) f_{u, \omega_{2}-\lambda}\left(\sigma_{1}, \tau_{2}\right) d \lambda \\
\quad+O\left(b_{\mathrm{f}, T} \log \left(b_{\mathrm{t}, T} T\right)\right)+O\left(b_{\mathrm{t}, T}^{2} b_{\mathrm{f}, T}\right)+O\left(\left(b_{\mathrm{t}, T} b_{\mathrm{f}, T} T\right)^{-1}\right) .
\end{array}
$$

The error terms will tend to zero under Assumption 3.5.7. Since the product of the two kernels in the first integral is exactly zero whenever $\left|\lambda-\left(\omega_{1}-\omega_{2}\right)\right|>b_{\mathrm{f}, T}$ or $\lambda>b_{\mathrm{f}, T}$, the first integral vanishes for large enough $T$ unless $\omega_{1}=\omega_{2}$. For $\omega_{1}=\omega_{2}$, the integral in the first term becomes

$$
\int_{-\pi}^{\pi} K_{\mathrm{f}, T}(-\lambda) K_{\mathrm{f}, T}(\lambda) f_{u, \omega_{1}+\lambda}\left(\tau_{1}, \tau_{2}\right) f_{u,-\omega_{1}-\lambda}\left(\sigma_{1}, \sigma_{2}\right) d \lambda
$$

and further by symmetry of the kernel

$$
=\int_{-\pi}^{\pi} K_{\mathrm{f}, T}(\lambda)^{2} f_{u, \omega_{1}+\lambda}\left(\tau_{1}, \tau_{2}\right) f_{u,-\omega_{1}-\lambda}\left(\sigma_{1}, \sigma_{2}\right) d \lambda .
$$

We note that $\left\|K_{\mathrm{f}}\right\|_{2}^{-2} K_{\mathrm{f}, T}(\lambda)^{2}$ satisfies the properties of an approximate identity (e.g., Edwards, 1967). Hence application of Lemma F.15 of Panaretos and Tavakoli (2013b), which covers approximate identities in a functional setting, yields that the integral converges to

$$
\left\|K_{\mathrm{f}}\right\|_{2}^{2} f_{u, \omega_{1}}\left(\tau_{1}, \tau_{2}\right) f_{u,-\omega_{1}}\left(\sigma_{1}, \sigma_{2}\right)
$$

with respect to $\|\cdot\|_{2}$. Since the integral in the second term in 3.49 vanishes unless $\omega_{1}=-\omega_{2}$, we can apply a similar argument, which proves the proposition. 
Corollary 3.5.9. Under the conditions of Theorem 3.5.6 and Assumption 3.5.7, we have

$$
\left\|\operatorname{cov}\left(\hat{\mathcal{F}}_{u, \omega_{1}}^{(T)}, \hat{\mathcal{F}}_{u, \omega_{2}}^{(T)}\right)\right\|_{2}=O\left(\frac{1}{b_{\mathrm{f}, T} b_{\mathrm{t}, T} T}\right)
$$

uniformly in $\omega_{1}, \omega_{2} \in[-\pi, \pi]$ and $u \in[0,1]$.

Proof. Since the frequency kernel satisfies $\left\|K_{\mathrm{f}, T}\right\|_{\infty}=O\left(\frac{1}{b_{\mathrm{f}, T}}\right)$ and $\left\|K_{\mathrm{f}, T}\right\|_{1}=1$, it is easy to see that

$$
\sup _{\omega}\left|\int_{-\pi}^{\pi} K_{\mathrm{f}, T}(\omega+\lambda) K_{\mathrm{f}, T}(\lambda) d \lambda\right|=O\left(\frac{1}{b_{\mathrm{f}, T}}\right) .
$$

Together with the fact that $\left\|\mathcal{F}_{u, \omega}\right\|_{2}$ is uniformly bounded in $u$ and $\omega$, the result then follows directly from (3.49).

Remark 3.5.10. If $\left\{\varepsilon_{t}\right\}$ is mean square continuous, $\left\|f_{\lambda_{1}, \cdots, \lambda_{k-1}}^{\varepsilon}\right\|_{\infty}<\infty$, and the transfer operator satisfies $\mathcal{A}_{t, \omega}^{(T)} \in \mathcal{B}_{2}$ with integral kernel that is continuous in its functional arguments for $k=2,4$ then Theorem 3.5.4, Theorem 3.5.6, Proposition 3.5.8, and Corollary 3.5.9 hold in uniform norm. For general transfer operators $\mathcal{A}_{t, \omega}^{(T)} \in \mathcal{B}_{\infty}$, the corresponding kernel $a_{t, \omega}^{(T)}$ is not necessarily well-defined or at least not bounded in $L^{2}$. However, for transfer operators that are elements of $\mathcal{B}_{2}$, that is, $\mathcal{A}_{t, \omega}^{(T)}=c I_{H}+\Upsilon_{t, T}^{\omega}$, where $c \in \mathbb{C}$ and $\Upsilon_{t, T}^{\omega} \in S_{2}(H)$, the result will also hold in uniform norm given the kernel of the Hilbert-Schmidt component is continuous in its functional arguments. In particular, for transfer operators $\mathcal{A}_{t, \omega}^{(T)} \in \mathcal{B}_{2}$, we can still use kernel notation if one takes as kernel of $I_{H}$ the Dirac delta function such that $I_{H} x(\tau)=\int_{0}^{1} \delta(\tau-\sigma) x(\sigma) d \sigma$. To see this, consider for example the term

$$
\begin{aligned}
{\left[\left(c I_{H}\right.\right.} & \left.\left.\otimes \Upsilon_{\lfloor u T+s / 2\rfloor, T}^{\omega}\right) \mathcal{F}_{\omega}^{\varepsilon}\right](\tau, \sigma) \\
& =c \int_{[0,1]^{2}} \delta\left(\tau-\mu_{1}\right) f_{\omega}^{\varepsilon}\left(\mu_{1}, \mu_{2}\right) \overline{\Upsilon_{\lfloor u T+s / 2\rfloor, T}^{\omega}\left(\sigma, \mu_{2}\right)} d \mu_{1} d \mu_{2} \\
& =c\left[\mathcal{F}_{\omega}^{\varepsilon}\left(\Upsilon_{\lfloor u T+s / 2\rfloor, T}^{\omega}\right)^{\dagger}\right](\tau, \sigma)=c\left[\Upsilon_{\lfloor u T+s / 2\rfloor, T}^{\omega} \mathcal{F}_{\omega}^{\varepsilon}\right](\sigma, \tau),
\end{aligned}
$$

where we used that the kernel of the conjugate operator is given by $a(\tau, \sigma)^{\dagger}=\overline{a(\sigma, \tau)}$. Note that this is consistent with the identity 3.59 in Definition A.1.2.

Theorem 3.5.11 (Convergence in integrated mean square). Under the conditions of Theorem 3.5.6 and bandwidths that satisfy Assumption 3.5.7, the spectral density operator is consistent in integrated mean square. More precisely, we have

$$
\begin{aligned}
\operatorname{IMSE}\left(\hat{\mathcal{F}}_{u, \omega}^{(T)}\right) & =\int_{-\pi}^{\pi} \mathbb{E}\left\|\hat{\mathcal{F}}_{u, \omega}^{(T)}-\mathcal{F}_{u, \omega}\right\|_{2}^{2} d \omega \\
& =O\left(\left(b_{\mathrm{t}, T} b_{\mathrm{f}, T} T\right)^{-1}\right)+o\left(b_{\mathrm{t}, T}^{2}+b_{\mathrm{f}, T}^{2}+\left(b_{\mathrm{t}, T} T\right)^{-1} \log \left(b_{\mathrm{t}, T} T\right)\right) .
\end{aligned}
$$

Since it is uniform in $\omega \in \Pi$, we have point-wise mean square convergence where the error also satisfies $\mathbb{E}\left\|\hat{\mathcal{F}}_{u, \omega}^{(T)}-\mathcal{F}_{u, \omega}\right\|_{2}^{2}=O\left(\frac{1}{b_{\mathrm{t}} b_{\mathrm{f}} T}\right)+o\left(b_{\mathrm{t}}^{2}+b_{\mathrm{f}}^{2}+\frac{\log b_{\mathrm{t}} T}{b_{\mathrm{t}} T}\right)$. 
Proof of Theorem 3.5.11. The proof follows straightforwardly from decomposing the above in terms of its variance and its squared bias. That is,

$$
\begin{aligned}
\int_{-\pi}^{\pi} \mathbb{E}\left\|\hat{\mathcal{F}}_{u, \omega}^{(T)}-\mathbb{E} \hat{\mathcal{F}}_{u, \omega}^{(T)}+\mathbb{E} \hat{\mathcal{F}}_{u, \omega}^{(T)}-\mathcal{F}_{u, \omega}\right\|_{2}^{2} d \omega \\
\quad=\int_{-\pi}^{\pi} \mathbb{E}\left\|\hat{\mathcal{F}}_{u, \omega}^{(T)}-\mathbb{E} \hat{\mathcal{F}}_{u, \omega}^{(T)}\right\|_{2}^{2} d \omega+\int_{-\pi}^{\pi} \mathbb{E}\left\|\mathbb{E} \hat{\mathcal{F}}_{u, \omega}^{(T)}-\mathcal{F}_{u, \omega}\right\|_{2}^{2} d \omega .
\end{aligned}
$$

The cross term cancels which is easily seen by noting that $\mathbb{E}\left(\hat{\mathcal{F}}_{u, \omega}^{(T)}-\mathbb{E}\left(\hat{\mathcal{F}}_{u, \omega}^{(T)}\right)\right)=O_{H}$ and hence

$$
\mathbb{E}\left(\left\langle\hat{\mathcal{F}}_{u, \omega}^{(T)}-\mathbb{E}\left(\hat{\mathcal{F}}_{u, \omega}^{(T)}\right), \mathbb{E}\left(\hat{\mathcal{F}}_{u, \omega}^{(T)}\right)-\mathcal{F}_{u, \omega}\right\rangle_{H \otimes H}\right)=0
$$

for all $u \in[0,1]$ and $\omega \in[-\pi, \pi]$. Now, for the first term of (4.60), we have by the properties of the Hilbert-Schmidt norm and, accordingly, by Corollary 3.5.9 that

$$
\int_{-\pi}^{\pi} \mathbb{E}\left\|\hat{\mathcal{F}}_{u, \omega}^{(T)}-\mathbb{E} \hat{\mathcal{F}}_{u, \omega}^{(T)}\right\|_{2}^{2} d \omega=\int_{-\pi}^{\pi} \int_{[0,1]^{2}} \operatorname{var}\left(\hat{f}_{u, \omega}^{(T)}(\tau, \sigma)\right) d \tau d \sigma d \omega=O\left(\frac{1}{b_{\mathrm{t}} b_{\mathrm{f}} T}\right) .
$$

Considering the second term of (4.60), Theorem 3.5.6 yields

$$
\begin{aligned}
\int_{-\pi}^{\pi} \| \mathcal{F}_{u, \omega}-\mathbb{E}\left(\hat{\mathcal{F}}_{u, \omega}^{(T)} \|_{2}^{2} d \omega\right. & =\int_{-\pi}^{\pi} \int_{[0,1]^{2}}\left|\mathbb{E} \hat{f}_{u, \omega}^{(T)}(\tau, \sigma)-f_{u, \omega}(\tau, \sigma)\right|^{2} d \tau d \sigma d \omega \\
& =O\left(b_{\mathrm{t}}^{2}+b_{\mathrm{f}}^{2}+\frac{\log b_{\mathrm{t}} T}{b_{\mathrm{t}} T}\right)^{2}
\end{aligned}
$$

\subsubsection{Weak convergence of the empirical process}

The results of the previous section give rise to investigating the limiting distribution of $\hat{f}_{u, \omega_{j}}^{(T)}$, the local estimator of the spectral density kernel and operator. We will show that for appropriate decay rates of the bandwidths, joint convergence to complex Gaussian elements in $L^{2}\left([0,1]^{2}, \mathbb{C}\right)$ can be established.

In our setting, we consider the sequence of random elements $\left(\hat{E}_{u, \omega}^{(T)}(\tau, \sigma)\right)_{T \in \mathbb{N}}$ in $L^{2}\left([0,1]^{2}, \mathbb{C}\right)$, where

$$
\hat{E}_{u, \omega}^{(T)}(\tau, \sigma)=\sqrt{b_{\mathrm{t}, T} b_{\mathrm{f}, T} T}\left(\hat{f}_{u, \omega}^{(T)}(\tau, \sigma)-\mathbb{E}\left[\hat{f}_{u, \omega}^{(T)}(\tau, \sigma)\right]\right)
$$

for $\tau, \sigma \in[0,1]$ and fixed $\omega \in[-\pi, \pi]$ and $u \in[0,1]$. In order to establish convergence in $L^{2}\left([0,1]^{2}, \mathbb{C}\right)$, it is more appropriate to consider the representation of $\hat{E}_{u, \omega}^{(T)}$ with respect to some orthonormal basis. For this, let $\left\{\psi_{m}\right\}_{m \in \mathbb{N}}$ be an orthonormal basis of $H$. Then $\left\{\psi_{m n}\right\}_{m, n \in \mathbb{N}}$ with $\psi_{m n}=\psi_{m} \otimes \psi_{n}$ forms an orthonormal basis of $L^{2}\left([0,1]^{2}, \mathbb{C}\right)$, and $\hat{E}_{u, \omega}^{(T)}$ equals

$$
\hat{E}_{u, \omega}^{(T)}=\sum_{m, n \in \mathbb{N}}\left\langle\hat{E}_{u, \omega}^{(T)}, \psi_{m n}\right\rangle \psi_{m n} .
$$

Hence, the distribution of $\hat{E}_{u, \omega}^{(T)}$ is fully characterized by the finite-dimensional distribution of the coefficients of its basis representation. Furthermore, weak convergence 
of $\hat{E}_{u, \omega}^{(T)}$ will follow from the weak convergence of $\left(\left\langle\hat{E}_{u, \omega}^{(T)}, \psi_{m n}\right\rangle\right)_{m, n \in \mathbb{N}}$ in the sequence space $\ell_{\mathbb{C}}^{2}$.

Subsequently, we identify $\hat{E}_{u, \omega}^{(T)}$ with its dual $\left(\hat{E}_{u, \omega}^{(T)}\right)^{*} \in L^{2}\left([0,1]^{2}, \mathbb{C}\right)^{*}$ and write

$$
\hat{E}_{u, \omega}^{(T)}(\phi)=\left\langle\hat{E}_{u, \omega}^{(T)}, \phi\right\rangle
$$

for all $\phi \in L^{2}\left([0,1]^{2}, \mathbb{C}\right)$.

To show convergence to a Gaussian functional process, we make use of the following result by Cremers and Kadelka (1986), which weakens the tightness condition usually employed to prove weak convergence and generalizes earlier results by Grinblat (1976).

Lemma 3.5.12. Let $(T, \mathcal{B}, \mu)$ be a measure space, let $(E,|\cdot|)$ be a Banach space, and let $\left(X_{n}\right)_{n \in \mathbb{N}}$ be a sequence of random elements in $L_{E}^{p}(T, \mu)$ such that

(i) the finite-dimensional distributions of $X_{n}$ converge weakly to those of a random element $X_{0}$ in $L_{E}^{p}(T, \mu)$ and

(ii) $\limsup _{n \rightarrow \infty} \mathbb{E}\left\|X_{n}\right\|_{p}^{p} \leqslant \mathbb{E}\left\|X_{0}\right\|_{p}^{p}$.

Then $X_{n}$ converges weakly to $X_{0}$ in $L_{E}^{p}(T, \mu)$.

In our setting, the weak convergence of the process $\hat{E}_{u, \omega}^{(T)}$ in $L^{2}\left([0,1]^{2}, \mathbb{C}\right)$ will follow from the joint convergence of $\hat{E}_{u, \omega}^{(T)}\left(\psi_{m_{1}, n_{1}}\right), \ldots, \hat{E}_{u, \omega}^{(T)}\left(\psi_{m_{k}, n_{k}}\right)$ for all $k \in \mathbb{N}$ and the condition

$$
\mathbb{E}\left\|\hat{E}_{u, \omega}^{(T)}\right\|_{2}^{2}=\sum_{m, n \in \mathbb{N}} \mathbb{E}\left|\hat{E}_{u, \omega}^{(T)}\left(\psi_{m n}\right)\right|^{2} \rightarrow \sum_{m, n \in \mathbb{N}} \mathbb{E}\left|E_{u, \omega}\left(\psi_{m n}\right)\right|^{2}=\mathbb{E}\left\|E_{u, \omega}\right\|_{2}^{2}
$$

as $T \rightarrow \infty$. In contrast, Panaretos and Tavakoli (2013b) employ the slightly stronger condition

$$
\left|\hat{E}_{u, \omega}^{(T)}\left(\psi_{m n}\right)\right|^{2} \leqslant \phi_{m n}
$$

for all $T \in \mathbb{N}$ and $m, n \in \mathbb{N}$ and some sequence $\left(\phi_{m n}\right) \in \ell^{1}$. In fact, the condition corresponds in our setting to the one given in Grinblat (1976). Finally, we note that condition (3.51) is sufficient for our purposes, but recently it has been shown (Bogachev and Miftakhov, 2015) that it can be further weakened to

$$
\sup _{T \in \mathbb{N}} \mathbb{E}\left\|\hat{E}_{u, \omega}^{(T)}\right\|_{2}^{2}<\infty .
$$

For the convergence of the finite-dimensional distributions, we show convergence of the cumulants of all orders to that of the limiting process. For the first and second order cumulants of $\hat{E}_{u, \omega}^{(T)}\left(\psi_{m n}\right)$, this follows from Theorem 3.5.6. It therefore remains to show that all cumulants of higher order vanish asymptotically.

Proposition 3.5.13. Under the conditions of Theorem 3.5.6, we have for all $u \in$ $[0,1]$ and for all $\omega_{i} \in[-\pi, \pi]$ and $m_{i}, n_{i} \in \mathbb{N}$ for $i=1, \ldots, k$, and for all $k \geqslant 3$

$$
\operatorname{cum}\left(\hat{E}_{u, \omega_{1}}^{(T)}\left(\psi_{m_{1} n_{1}}\right), \ldots, \hat{E}_{u, \omega_{k}}^{(T)}\left(\psi_{m_{k} n_{k}}\right)\right)=o(1)
$$

as $T \rightarrow \infty$. 
The proof of the proposition can be found in Section A.4.3 of the appendix. The distributional properties of the functional process are then given in the following theorem.

Theorem 3.5.14 (Weak convergence). Let $\left\{X_{t, T}\right\}$ be a locally stationary functional process satisfying Proposition 3.2.4 with $\mathcal{A}_{t, \omega}^{(T)} \in \mathcal{B}_{\infty}$ and $\sup _{\omega_{1}, \ldots, \omega_{k-1}}\left\|\mathcal{F}_{\omega_{1}, \ldots, \omega_{k-1}}^{\varepsilon}\right\|_{2}<$ $\infty$ for $k=2,4$. Additionally, let the operator-valued function $\mathcal{A}_{u, \omega}$ be Hölder continuous of order $\alpha>1 / 2$ and twice continuously differentiable in both $u$ and $\omega$. Then for bandwidths that satisfy Assumption 3.5.7

$$
\left(\hat{E}_{u, \omega_{j}}^{(T)}\right)_{j=1, \ldots, J} \stackrel{\mathcal{D}}{\rightarrow}\left(E_{u, \omega_{j}}\right)_{j=1, \ldots, J}
$$

where $E_{u, \omega_{j}}, j=1, \ldots, J$, are jointly Gaussian elements in $L_{\mathbb{C}}^{2}\left([0,1]^{2}\right)$ with means $\mathbb{E}\left(E_{u, \omega_{i}}\left(\psi_{m n}\right)\right)=0$ and covariances

$$
\begin{array}{r}
\operatorname{cov}\left(E_{u, \omega_{i}}\left(\psi_{m n}\right), E_{u, \omega_{j}}\left(\psi_{m^{\prime} n^{\prime}}\right)\right) \\
=2 \pi\left\|K_{\mathrm{t}}\right\|_{2}^{2}\left\|K_{\mathrm{f}}\right\|_{2}^{2}\left[\eta\left(\omega_{i}-\omega_{j}\right)\left\langle\mathcal{F}_{u, \omega_{i}} \psi_{m^{\prime}}, \psi_{m}\right\rangle\left\langle\mathcal{F}_{u,-\omega_{i}} \psi_{n^{\prime}}, \psi_{n}\right\rangle\right. \\
\left.+\eta\left(\omega_{i}+\omega_{j}\right)\left\langle\mathcal{F}_{u, \omega_{i}} \psi_{n^{\prime}}, \psi_{m}\right\rangle\left\langle\mathcal{F}_{u,-\omega_{i}} \psi_{m^{\prime}}, \psi_{n}\right\rangle\right]
\end{array}
$$

for all $i, j \in 1, \ldots, J$ and $m, m^{\prime}, n, n^{\prime} \in \mathbb{N}$.

Proof of Theorem 3.5.14. For condition 3.51, we note that

$$
\mathbb{E}\left\|\hat{E}_{u, \omega}^{(T)}\right\|_{2}^{2}=\int_{[0,1]^{2}} \operatorname{var}\left(\hat{E}_{u, \omega}^{(T)}(\tau, \sigma)\right) d \tau d \sigma=b_{\mathrm{t}, T} b_{\mathrm{f}, T} T\left\|\operatorname{var}\left(\mathcal{F}_{u, \omega}^{(T)}\right)\right\|_{2}^{2} .
$$

and it therefore is satisfied by Theorem 3.5.6. Together with the convergence of the finite-dimensional distributions this proves the asserted weak convergence.

\subsection{Numerical simulations}

To illustrate the performance of the estimator in finite samples, we consider a timevarying functional time series with representation

$$
X_{t, T}=B_{\frac{t}{T}, 1}\left(X_{t-1, T}\right)+\varepsilon_{t},
$$

where $B_{\frac{t}{T}, 1} \in \mathcal{B}_{\infty}$ for all $t$ and $T$ and where $\left\{\varepsilon_{t}\right\}$ is a collection of independent innovation functions. In order to generate the process, let $\left\{\psi_{i}\right\}_{i \in \mathbb{N}}$ be an orthonormal basis of $H$ and denote the vector of the first $k$ Fourier coefficients of $X_{t, T}$ by $\boldsymbol{X}_{t}^{(T)}=$ $\left(\left\langle X_{t, T}, \psi_{1}\right\rangle, \ldots,\left\langle X_{t, T}, \psi_{k}\right\rangle\right)^{\prime}$. Similar to Hörmann et al. (2015), we exploit that the linearity of the autoregressive operator implies the first $k$ Fourier coefficients, for $k$ large, approximately satisfy a $\operatorname{VAR}(1)$ equation. That is,

$$
\boldsymbol{X}_{t}^{(T)} \approx \mathfrak{B}_{\frac{t}{T}, 1} \boldsymbol{X}_{t-1}^{(T)}+\varepsilon_{t} \quad \forall t, T,
$$


where $\varepsilon_{t}=\left(\left\langle\varepsilon_{t}, \psi_{1}\right\rangle, \ldots,\left\langle\varepsilon_{t}, \psi_{k}\right\rangle\right)^{\prime}$ and $\mathfrak{B}_{\frac{t}{T}, 1}=\left(\left\langle B_{\frac{t}{T}, 1}\left(\psi_{i}\right), \psi_{j}\right\rangle, 1 \leqslant i, j \leqslant k\right)$. Correspondingly, the local spectral density kernel will satisfy

$$
f_{u, \omega}^{(T)}(\tau, \sigma) \approx \lim _{k \rightarrow \infty} \sum_{i, j=1}^{k} \mathfrak{f}_{u, \omega, i, j}^{(T)} \psi_{i}(\tau) \psi_{j}(\sigma),
$$

where $\mathfrak{f}_{u, \omega}^{(T)}$ is the spectral density matrix of the Fourier coefficients in (3.56). Implementation was done in $\mathrm{R}$ together with the fda package. For the simulations, we chose the Fourier basis functions on $[0,1]$. The construction of the estimator in (3.44) requires specification of smoothing kernels and corresponding bandwidths in time as well as frequency direction. Although the choice of the smoothing kernels usually does not affect the performance significantly, bandwidth selection is a well-known problem in nonparametric statistics. As seen from Theorem 3.5.6, both bandwidths influence the bias-variance relation. Depending on the persistence of the autoregressive process a smaller bandwidth in frequency direction is desirable around the peak (at $\lambda=0$ for the above process), while slow changes in time direction allow for tapering (i.e., smoothing in time direction) over more functional observations. It would therefore be of interest to develop an adaptive procedure as proposed in van Delft and Eichler (2015) to select the bandwidth parameters. Investigation of this is however beyond the scope of the current chapter. In the examples below, the bandwidths were set fixed to $b_{\mathrm{t}, T}=T^{-1 / 6}$ and $b_{\mathrm{f}, T}=2 T^{-1 / 5}-b_{\mathrm{t}, T}$. We chose as smoothing kernels

$$
K_{\mathrm{t}}(x)=K_{\mathrm{f}}(x)=6\left(\frac{1}{4}-x^{2}\right) \quad x \in\left[-\frac{1}{2}, \frac{1}{2}\right],
$$

which have been shown to be optimal in the time series setting (Dahlhaus, 1996b).

In order to construct the matrix $\mathfrak{B}_{\frac{t}{T}, 1}$, we first generate a matrix $A_{u}$ with entries that are mutually independent Gaussian where the $(i, j)$-th entry has variance

$$
u i^{-2 c}+(1-u) e^{-i-j} .
$$

The entries will tend to zero as $i, j \rightarrow \infty$, because the operator $B_{\frac{t}{T}, 1}$ is required to be bounded. The matrix $\mathfrak{B}_{\frac{t}{T}, 1}$ is consequently obtained as $\mathfrak{B}_{\frac{t}{T}, 1}=\eta A_{u} /\left\|A_{u}\right\|_{\infty}$. The value of $\eta$ thus determines the persistence of the process. Additionally, the collection of innovation functions $\left\{\varepsilon_{t}\right\}$ is specified as a linear combination of the Fourier basis functions with independent zero-mean Gaussian coefficients such that the $l$-th coefficient $\left\langle\varepsilon_{t}, \psi_{l}\right\rangle$ has variance $1 /[(l-1.5) \pi]^{2}$. The parameters were set to $c=3$ and $\eta=0.4$. To visualize the variability of the estimator, tables 3.1-3.2 depict the amplitude of the true spectral density kernel of the process for various values of $u$ and $\lambda$ with 20 replications of the corresponding estimator superposed for different sample sizes $T$. For each row, the same level curves were used where each level curve has the same color-coding within that row. Table 3.1 gives the different levels for the estimator around the peak in frequency direction, while table 3.2 provides contour plots further away from the peak. Increasing the sample size leads to less variability, as can be seen from the better aligned contour lines. It can 
also be observed that the estimates become more stable as we move further away from the peak. Nevertheless, the peaks and valleys are generally reasonably well captured even for the contour plots in the area around the peak.

As a second example, we consider a $\operatorname{FAR}(2)$ with the location of the peak varying with time. More specifically, the Fourier coefficients are now obtained by means of a $\operatorname{VAR}(2)$

$$
\boldsymbol{X}_{t}^{(T)}=\mathfrak{B}_{\frac{t}{T}, 1} \boldsymbol{X}_{t-1}^{(T)}+\mathfrak{B}_{\frac{t}{T}, 2} \boldsymbol{X}_{t-2}^{(T)}+\varepsilon_{t},
$$

where $\mathfrak{B}_{\frac{t}{T}, 1}=\eta_{u, 1} A_{u, 1} /\left\|A_{u, 1}\right\|_{\infty}$ and $\mathfrak{B}_{\frac{t}{T}, 2}=\eta_{u, 2} A_{u, 2} /\left\|A_{u, 2}\right\|_{\infty}$. The entries of the matrices $A_{u, 1}$ and $A_{u, 2}$ are mutually independent and are generated such that $\left[A_{u, 1}\right]_{i, j}=\mathcal{N}\left(0, e^{-(i-3)-(j-3)}\right)$ and $\left[A_{u, 2}\right]_{i, j}=\mathcal{N}\left(0,\left(i^{8 / 2}+j^{2 / 2}\right)^{-1}\right)$, respectively. The norms are specified as

$$
\eta_{u, 1}=0.4 \cos (1.5-\cos (\pi u)) \text { and } \eta_{u, 2}=-0.5 .
$$

This will result in the peak to be located at $\lambda=\arccos (0.3 \cos [1.5-\cos (\pi u)])$. The collection of innovation functions $\left\{\varepsilon_{t}\right\}$ is chosen such that the $l$-th coefficient $\left\langle\varepsilon_{t}, \psi_{l}\right\rangle$ has variance $1 /[(l-2.65) \pi]^{2}$. Table 3.3 provides the contour plots for different local time values where the frequency was set to $\lambda=1.5-\cos (\pi u)$, i.e., the direction in which most change in time direction is visible in terms of amplitude. Overall, the method seems to identify both the location and magnitude of the peaks and valleys very well, where again the variability clearly reduces for $T>512$. This is promising for the application to real data. For the value $u=0.5$, one is really close to the location of a peak and observe wrongful detection of a small peak in the middle of the contour plot. This is an indication some over-smoothing occurs which, to some extent, is difficult to prevent for autoregressive models, even in the stationary time series case. 

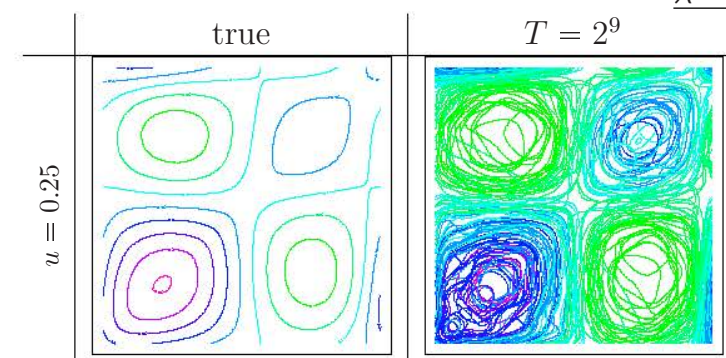

$\underline{\lambda=0}$
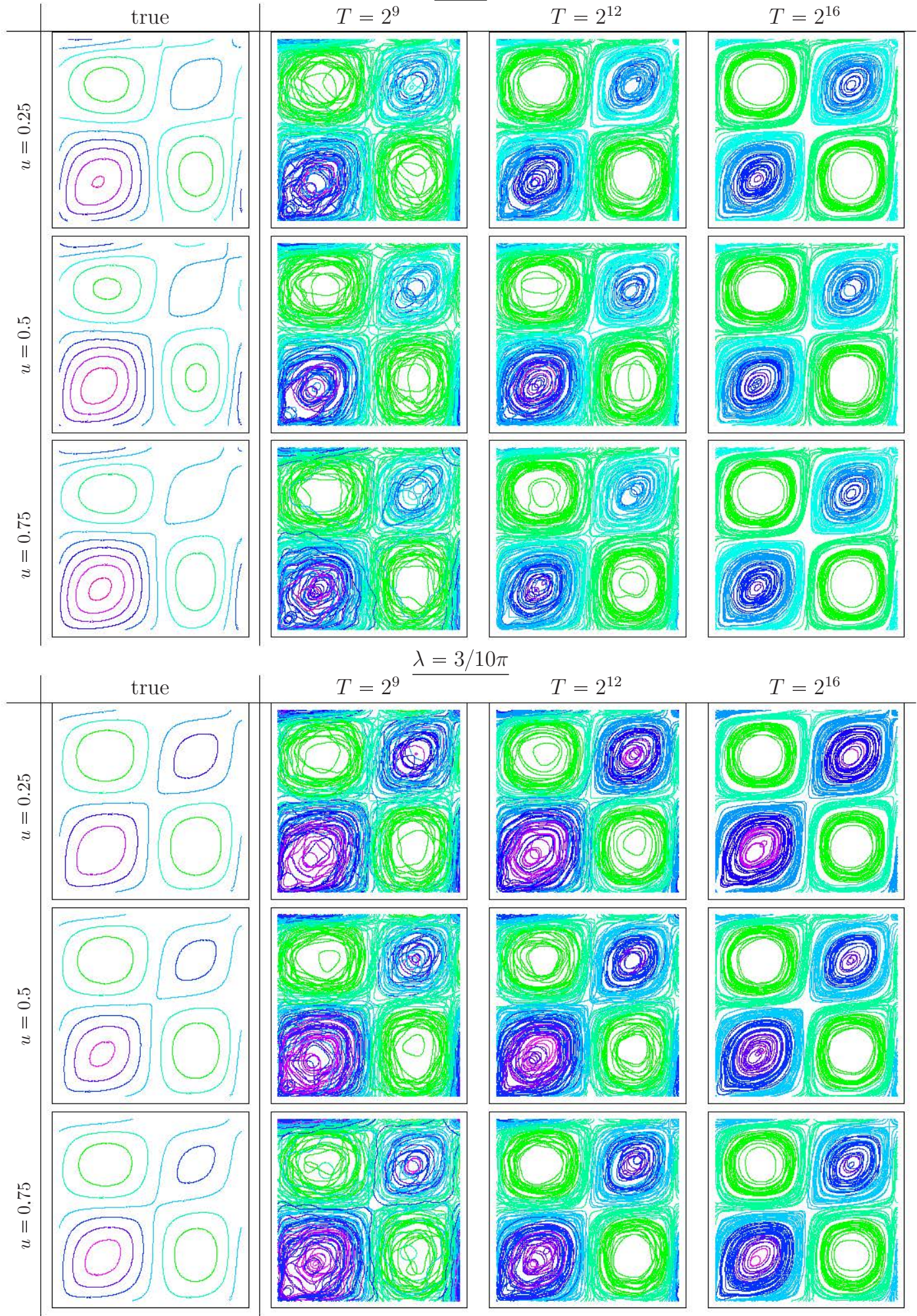

Table 3.1: Contour plots of the true and estimated spectral density of the FAR(1) at different time points at frequencies $\lambda=0$ and $\lambda=\frac{3}{10} \pi$. 


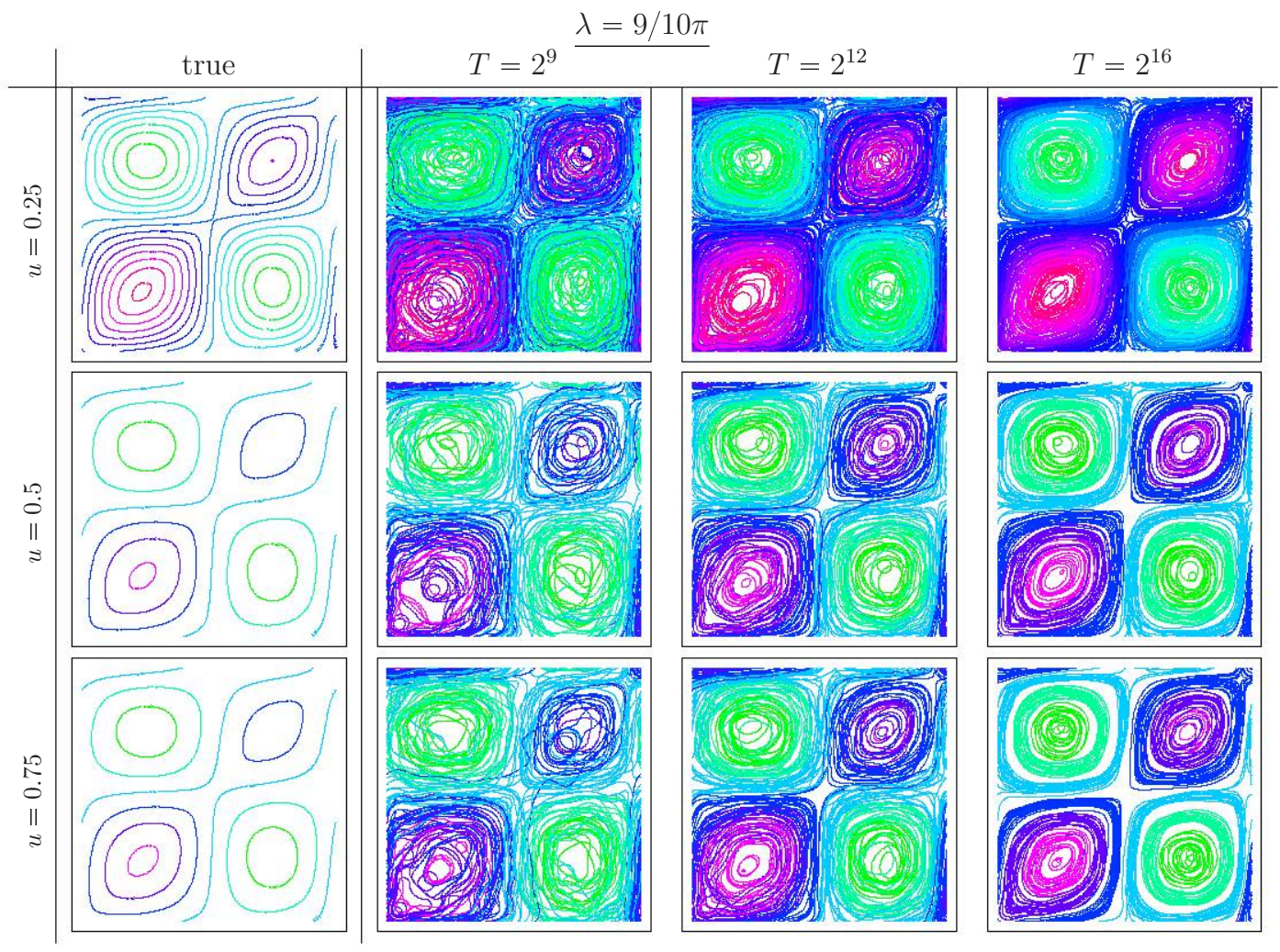

Table 3.2: Contour plots of the true and estimated spectral density of the FAR(1) at different time points at frequency $\lambda=\frac{9}{10} \pi$. 

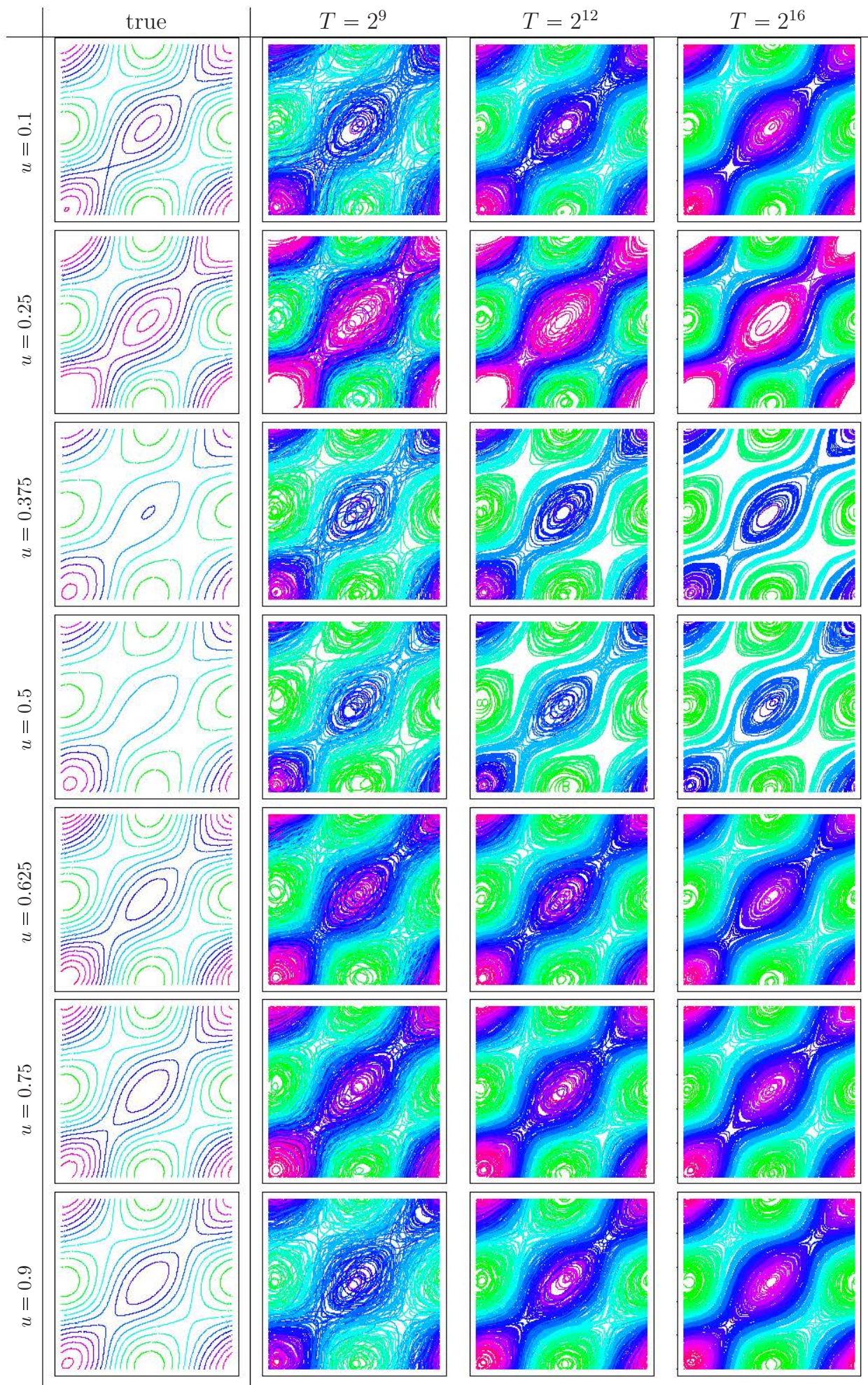

Table 3.3: Contour plots of the true and estimated spectral density of the $\operatorname{FAR}(2)$ at different time points for $\lambda=1.5-\cos (\pi u)$. 


\subsection{Conclusion}

This chapter provided a theoretical framework for meaningful statistical inference of functional time series of which the dynamics change slowly over time. For this, we introduced the notion of local stationarity for time series that take values in infinitedimensional separable Hilbert spaces. Because it is often not clear what specific model a functional time series follows, a well-defined spectral theory can especially be important to capture the dependence structure. Particular attention is therefore paid to a class of functional locally stationary processes for which a time-varying functional spectral representation exists. The second-order characteristics of processes belonging to this class are completely captured by the so-called time-varying spectral density operator. Analogously, the higher order dependence structure in terms of cumulant tensors can be given by the corresponding higher order cumulant spectral operators. From the triangular array that constitutes the locally stationary process, the time-varying spectral density operator has been demonstrated to be uniquely defined. This is a useful property that will be further explored in future research.

Time-varying functional ARMA processes were introduced and shown to have a well-defined time-varying functional Cramér representation. An important aspect of this result has been the determination of the existence of a causal solution of timevarying functional autoregressive processes. Weakly stationary functional (ARMA) processes are comprised as a special case of the introduced class. The introduced class can therefore be seen to provide a direct generalization of the Cramér representation to nonstationary functional processes. More generally, the asymptotic theory introduced reduces to classical asymptotic theory for functional time series in case the second-order characteristics are temporally constant. This generalization makes an easy comparison possible of the behavior of processes with changing characteristics versus those for which these remain constant. Such information is crucial for the correct use of statistical methods and tools and hence for correct inference. The next chapter is devoted to the issue of determining whether the assumption of weak stationarity is justifiable.

The last section of this chapter was concerned with the estimation of the timevarying spectral density operator. To construct the estimator, we considered the tensor product of tapered versions of the functional Discrete Fourier Transform. The taper acts as a bandwidth in time direction. The properties of the resulting segmented periodogram operator, a functional generalization of the segmented periodogram matrix, were derived and the operator was shown to behave in corroboration to what one would expect based on the results available in the finite-dimensional setting. To form consistent estimators, these operators were subsequently linearly transformed by smoothing them with a window function in frequency direction. Conditions on the bandwidths necessary for consistency were then studied and the asymptotic distribution was derived. The latter was done using a weaker tightness criterion than what is usually employed in the existing literature and allowed to show convergence to a Gaussian functional process without imposing stronger conditions on the Schatten $p$-class of the time-varying spectral density operator. Finite 
sample properties of the estimator were illustrated at the end of the chapter via a simulation study.

This chapter can be seen to form the basis for the development of statistical techniques and methods for the analysis of functional time series that require relaxation of the assumption of weak stationarity. Because the random elements are intrinsically infinite-dimensional, of direct interest would be the development of dimension reduction techniques. In particular, functional dynamic principal component analysis for nonstationary functional time series as well as the investigation of the validity and optimality of localized versions of the Karhunen-Loève expansion will be investigated in future work. 



\section{Appendix}

\section{A.1 Some operator theory}

We start with a general characterization of a tensor product of a finite sequence of vector spaces, which in particular holds for sequences of Hilbert spaces.

Definition A.1.1 (Algebraic tensor product of Banach spaces). Given a finite sequence of vector spaces $V_{1}, \ldots, V_{k}$ over an arbitrary field $\mathbb{F}$, we define the algebraic tensor product $V_{1} \otimes \cdots \otimes V_{k}$ as a vector space with a multi-linear map $V_{1} \times \cdots \times V_{k} \rightarrow W$ given by $\left(f_{1}, \ldots, f_{k}\right) \rightarrow\left(f_{1} \otimes \cdots \otimes f_{k}\right)$ such that, for every linear map $\mathcal{T}: V_{1} \times \cdots \times V_{k} \rightarrow W$, there is unique k-linear map $\tilde{\mathcal{T}}: V_{1} \times \cdots \times V_{k} \rightarrow W$ that satisfies

$$
\mathcal{T}\left(f_{1}, \ldots, f_{k}\right)=\tilde{\mathcal{T}}\left(f_{1} \otimes \cdots \otimes f_{k}\right)
$$

Here, uniqueness is meant up to isomorphisms. The tensor product can be viewed as a linearized version of the product space $V_{1} \times \cdots \times V_{k}$ satisfying equivalence relations of the form $a\left(v_{1}, v_{2}\right) \sim\left(a v_{1}, v_{2}\right) \sim\left(v_{1}, a v_{2}\right)$ where $a \in \mathbb{K}$ and $v_{1} \in V_{1}, v_{2} \in V_{2}$, which induce a quotient space. These relationships uniquely identify the points in the product space $V_{1} \times \ldots \times V_{k}$ that yield multi-linear relationships. In a way, the tensor product $\otimes_{j=1}^{k} V_{j}$ can thus be viewed as the 'freest' way to put the respective different vector spaces $V_{1}, \ldots, V_{k}$ together. We mention in particular that the algebraic tensor product satisfies the associative law, i.e., $\left(V_{1} \otimes V_{2}\right) \otimes V_{3}=V_{1} \otimes\left(V_{2} \otimes V_{3}\right)$, and hence it will often be sufficient to restrict attention to $k=2$.

The algebraic tensor product of two Hilbert spaces $H_{1}$ and $H_{2}$ is itself not a Hilbert space. We can however construct a Hilbert space by considering the inner product acting on $H_{1} \otimes H_{2}$ given by

$$
\left\langle x \otimes y, x^{\prime} \otimes y^{\prime}\right\rangle_{H_{1} \otimes H_{2}}=\left\langle x, x^{\prime}\right\rangle\left\langle y, y^{\prime}\right\rangle, \quad x, x^{\prime} \in H_{1}, y, y^{\prime} \in H_{2}
$$

and then taking the completion with respect to the induced norm $\|\cdot\|_{H_{1} \otimes H_{2}}$. The completed space, denoted by $H_{1} \widehat{\otimes} H_{2}$, is identifiable with the Hilbert-Schmidt operators and is referred to as the Hilbert Schmidt tensor product. Throughout this chapter, when reference is made to the tensor product space of Hilbert spaces, we mean the latter space. When no confusion can arise, we shall moreover abuse notation slightly and denote $H_{1} \widehat{\otimes} H_{2}$ simply by $H_{1} \otimes H_{2}$. 
Definition A.1.2. The tensor product $(A \otimes B) \in S_{P}(H) \otimes S_{p}(H) \cong S_{p}\left(S_{p}(H)\right)$ between two operators $A, B \in S_{p}(H)$ is defined as

$$
(A \otimes B)(x \otimes y)=A x \otimes B y,
$$

for $x, y \in H$. It follows straightforwardly from the property

$$
(x \otimes y) z=\langle z, y\rangle x, \quad z \in H,
$$

that for any $C \in S_{p}(H)$, we have the identity

$$
(A \otimes B) C=A C B^{\dagger},
$$

where $B^{\dagger}$ denote the adjoint operator of $B$.

Proposition A.1.3 (Hölder's Inequality for operators). Let $H$ be a separable Hilbert space and $A, B \in S_{\infty}(H)$. Then the composite operator $A B$ also defines a bounded linear operator over $H$, i.e., $A B \in S_{\infty}(H)$. This operation satisfies the associative law. Moreover, let $1 \leqslant p, q, r \leqslant \infty$, such that $\frac{1}{r}=\frac{1}{q}+\frac{1}{p}$. If $A \in S_{q}(H)$ and $B \in S_{p}(H)$ then $A B \in S_{r}(H)$ and

$$
\|A B\|_{r} \leqslant\|A\|_{q}\|B\|_{p} .
$$

Proposition A.1.4. Let $H=L_{\mathbb{C}}^{2}(T, \mu)$ be a separable Hilbert space, where $(T, \mu)$ is a measure space. The functions $a, b, c \in L_{\mathbb{C}}^{2}(T \times T, \mu \otimes \mu)$ induce operators $A, B, C$ on $H$ such that for all $x \in H$

$$
A x(\tau)=\int a\left(\tau, \mu_{1}\right) x\left(\mu_{1}\right) d \mu_{1},
$$

and the composition operator $A B$ has kernel

$$
[A B](\tau, \sigma)=\int a\left(\tau, \mu_{1}\right) b\left(\mu_{1}, \sigma\right) d \mu_{1},
$$

for all $\tau \in T \mu$-almost everywhere. The tensor product operator $(A \otimes B) \in S_{2}\left(S_{2}(H)\right)$ in composition with $C$ has kernel

$$
[(A \otimes B) C](\tau, \sigma)=\int_{\mathcal{D}} \int_{\mathcal{D}} a\left(\tau, \mu_{1}\right) \overline{b\left(\sigma, \mu_{2}\right)} c\left(\mu_{1}, \mu_{2}\right) d \mu_{1} d \mu_{2} .
$$

Because $(A \otimes B) C$ has a well defined kernel in $L_{\mathbb{C}}^{2}(T \times T, \mu \otimes \mu)$, it can moreover be viewed as an operator on $H$. Using identity (3.59), this is the operator $A C B^{\dagger}$, where $B^{\dagger}$ has kernel $b^{\dagger}\left(\mu_{2}, \sigma\right)=\overline{b\left(\sigma, \mu_{2}\right)}$.

Corollary A.1.5. Let $A_{i}, i=1, \cdots, k$ for $k$ finite belong to $S_{p}(H)$ and let

$$
\boldsymbol{\psi}=\left(\psi_{1} \otimes \cdots \otimes \psi_{k}\right)
$$

be an element of $\otimes_{i=1}^{k} H$. Then we have that the linear mapping

$$
\mathcal{A}=\left(A_{1} \otimes \ldots \otimes A_{k}\right)
$$

satisfies i) $\|\mathcal{A} \psi\|_{2}<\infty$ and ii) $\|\mathcal{A}\|_{p}<\infty$. 
Proof of Corollary A.1.5. For i), we have by proposition A.1.3,

$$
\begin{aligned}
& \|\mathcal{A} \boldsymbol{\psi}\|_{2}=\left\|\left(A_{1} \otimes \ldots \otimes A_{k}\right) \boldsymbol{\psi}\right\|_{2}=\left\|\left(A_{1} \otimes \ldots \otimes A_{k}\right)\right\|_{\infty}\|\boldsymbol{\psi}\|_{2} \\
& \leqslant\left\|\left(A_{2} \otimes \ldots \otimes A_{k}\right)\right\|_{\infty}\left\|A_{1}\right\|_{\infty}\|\boldsymbol{\psi}\|_{2} \\
& \leqslant \prod_{i=1}^{k}\left\|A_{i}\right\|_{\infty}\|\boldsymbol{\psi}\|_{2} \leqslant \prod_{i=1}^{k}\left\|A_{i}\right\|_{p}\|\boldsymbol{\psi}\|_{2}<\infty
\end{aligned}
$$

In case $p=2$, the latter equals $\prod_{i=1}^{k}\left\|a_{i}\right\|_{2}\|\boldsymbol{\psi}\|_{2}$ by proposition A.1.7. Property ii) holds since for any $A_{1}, A_{2} \in S_{p}(H)$, we have $\left\|A_{1} \otimes A_{2}\right\|_{p}=\left\|A_{1}\right\|\left\|_{p}\right\| A_{2} \|_{p}$. To illustrate the second property, observe that if $p=2$ we obtain

$$
\begin{aligned}
& \|\mathcal{A}\|_{2}^{2}=\left\|A_{1} \otimes \ldots \otimes A_{k}\right\|_{2}^{2}=\int_{[0,1]^{2 k}}\left|a_{1}\left(\tau_{1}, \mu_{1}\right) . . a_{k}\left(\tau_{k}, \mu_{k}\right)\right|^{2} d \tau_{1} . . d \tau_{k} d \mu_{1} . . d \mu_{k} \\
& =\int_{[0,1]^{2 k}} a_{1}\left(\tau_{1}, \mu_{1}\right) \overline{a_{1}\left(\tau_{1}, \mu_{1}\right)} . . a_{k}\left(\tau_{k}, \mu_{k}\right) \overline{a_{k}\left(\tau_{k}, \mu_{k}\right)} d \tau_{1} . . d \tau_{k} d \mu_{1} . . d \mu_{k} \\
& =\int_{[0,1]^{2}} a_{1}\left(\tau_{1}, \mu_{1}\right) \overline{a_{1}\left(\tau_{1}, \mu_{1}\right)} d \tau_{1}, d \mu_{1} . . \int_{[0,1]^{2}} a_{k}\left(\tau_{k}, \mu_{k}\right) \overline{a_{k}\left(\tau_{k}, \mu_{k}\right)} d \tau_{k} d \mu_{k} \\
& =\left\|a_{1}\right\|_{2}^{2} . .\left\|a_{k}\right\|_{2}^{2}<\infty .
\end{aligned}
$$

Proposition A.1.6 (Neumann series). Let $A$ be a bounded linear operator on $H$ and $I_{H}$ be the identity operator. If $\|A\|_{\infty}<1$, the operator $I_{H}-A$ has a unique bounded inverse on $H$ given by

$$
\left(I_{H}-A\right)^{-1}=\sum_{k=0}^{\infty} A^{k}
$$

If $A \in S_{2}(H)$ with $\|A\|_{2}<1$, then this equality holds in Hilbert-Schmidt norm.

Proof. We only show the case $A \in S_{2}(H)$. Note that the space $S_{2}(H)$ is a Hilbert space. Then for $m<n$,

$$
\left\|\sum_{k=0}^{m} A^{k}-\sum_{k=0}^{n} A^{k}\right\|_{2} \leqslant \sum_{k=m+1}^{n}\|A\|_{2}^{k} \leqslant \frac{\|A\|_{2}^{m+1}}{1-\|A\|_{2}},
$$

which shows that the partial sum forms a Cauchy sequence and hence has a limit $A^{*}$ in $S_{2}(H)$. Furthermore, we have

$$
\left(I_{H}-A\right) A^{*}=\lim _{n \rightarrow \infty}\left(I_{H}-A\right) \sum_{k=0}^{n} A^{n}=\lim _{n \rightarrow \infty}\left(I_{H}-A^{n+1}\right)=I_{H}
$$

in $S_{2}(H)$, which shows that $A^{*}$ is the inverse of $I_{H}-A$. 
Proposition A.1.7 (Hilbert-Schmidt operators as kernel operator). Let $H=L_{\mathbb{C}}^{2}(T, \mu)$ be a separable Hilbert space, where $(T, \mu)$ is a measure space, and let $A$ be an operator on $H$. Then $A \in S_{2}(H)$ if and only if it is an integral operator, that is, there exists a function $a \in L_{\mathbb{C}}^{2}(T \times T, \mu \otimes \mu)$ such that

$$
A x(\tau)=\int a(\tau, \sigma) x(\sigma) d \mu(\tau) d \mu(\sigma)
$$

for all $\tau \in T \mu$-almost everywhere. Moreover, we have $\|A\|_{2}=\|a\|_{2}$.

Proof. First, suppose $A$ is an integral operator on $H$ with kernel $a \in L_{\mathbb{C}}^{2}(T \times T, \mu \otimes \mu)$. Because $H$ is separable, it has a countable orthonormal basis $\left\{\psi_{n}\right\}_{n \in \mathbb{N}}$. For fixed $\tau \in M$, the function $a_{\tau}(\sigma)=a(\tau, \sigma)$ defines a measurable function on $L_{\mathbb{C}}^{2}(T, \mu)$. We can therefore write

$$
A \psi(\tau)=\int a(\tau, \sigma) \psi_{n}(\sigma) d \sigma=\left\langle a_{\tau}, \overline{\psi_{n}}\right\rangle
$$

Observe that $\left\{\overline{\psi_{n}}\right\}_{n \geqslant 1}$ also forms a orthonormal basis of $H$. An application of the Cauchy-Schwarz Inequality gives $\left|\left\langle a_{\tau}, \overline{\psi_{n}}\right\rangle\right|^{2} \leqslant\left\|a_{\tau}\right\|^{2}\left\|\overline{\psi_{n}}\right\|^{2}<\infty$ and therefore

$$
\sum_{n=1}^{m}\left|\left\langle a_{\tau}, \overline{\psi_{n}}\right\rangle\right|^{2} \leqslant \sum_{n=1}^{\infty}\left|\left\langle a_{\tau}, \overline{\psi_{n}}\right\rangle\right|^{2}=\left\|a_{\tau}\right\|_{2}^{2}<\infty,
$$

by Parseval's Identity. Hence, as a corollary of the Monotone and Dominated Convergence Theorem we find

$$
\begin{aligned}
\|A\|_{2}^{2} & =\sum_{n=1}^{\infty}\left\|A \psi_{n}\right\|^{2}=\lim _{m \rightarrow \infty} \sum_{n=1}^{m}\left|\left\langle a_{\tau}, \bar{\psi}_{n}\right\rangle\right|^{2} d \tau=\int \lim _{m \rightarrow \infty} \sum_{n=1}^{m}\left|\left\langle a_{\tau}, \bar{\psi}_{n}\right\rangle\right|^{2} d \tau \\
& =\int\left\|a_{\tau}\right\|^{2} d \tau=\iint|a(\tau, \sigma)|^{2} d \sigma d \tau=\|a\|_{2}^{2}<\infty,
\end{aligned}
$$

showing $A$ is Hilbert Schmidt and $\|A\|_{2}=\|a\|_{2}$. Now suppose $A$ is Hilbert Schmidt. In this case, we have by definition $\sum_{n=1}^{\infty}\left\|A \psi_{n}\right\|^{2}<\infty$ and consequently the series $\sum_{n=1}^{\infty} A \psi_{n}$ converges in $L_{\mathbb{C}}^{2}(T, \mu)$. Therefore the function

$$
a(\tau, \sigma):=\sum_{n=1}^{\infty} A \psi_{n}(\tau) \overline{\psi_{n}(\sigma)}
$$

will be well-defined on $L_{\mathbb{C}}^{2}(T \times T, \mu \otimes \mu)$. Hence, for any element $x \in L_{\mathbb{C}}^{2}(T, \mu)$, the Dominated Convergence Theorem yields

$$
\begin{aligned}
A x(\tau) & =A\left(\lim _{m \rightarrow \infty} \sum_{n=1}^{m}\left\langle x, \psi_{n}\right\rangle \psi_{n}\right)(\tau)=\lim _{m \rightarrow \infty} \sum_{n=1}^{m}\left\langle x, \psi_{n}\right\rangle A \psi_{n}(\tau) \\
& =\lim _{m \rightarrow \infty} \sum_{n=1}^{m}\left(\int x(\sigma) \overline{\psi_{n}(\sigma)} d \sigma\right) A \psi_{n}(\tau)=\lim _{m \rightarrow \infty}\left(\int x(\sigma) \sum_{n=1}^{m} \overline{\psi_{n}(\sigma)} A \psi_{n}(\tau) d \sigma\right) \\
& \int x(\sigma) \sum_{n \geqslant 1} \overline{\psi_{n}(\sigma)} A \psi_{n}(\tau) d \sigma=\int x(\sigma) a(\tau, \sigma) d \sigma .
\end{aligned}
$$


Theorem A.1.8 (Product Rule on Banach spaces). Let $E, F_{1}, F_{2}, G$ be Banach spaces and let $U \subset E$ be open. Suppose that $f: U \rightarrow F_{1}$, and $G: U \in F_{2}$ are Fréchet differentiable of order $k$. Let $Z(\cdot, \cdot): F_{1} \times F_{2} \rightarrow G$ be a continuous bilinear map. Then, $Z(f, g): U \rightarrow G$ is Fréchet differentiable of order $k$ and

$$
\frac{\partial Z}{\partial u}(f(u), g(u))=Z\left(\frac{\partial f(u)}{\partial u}, g(u)\right)+Z\left(f(u), \frac{\partial g(u)}{\partial u}\right) .
$$

For the proof, see for example Nelson (1969).

\section{A.2 Higher order dependence}

\section{A.2.1 Moment tensors on separable Hilbert spaces}

Let $X$ be a random element of the probability space $(\Omega, \mathscr{A}, \mathbb{P})$ that takes values in a separable Hilbert space $H$. More precisely, we endow $H$ with the topology induced by the norm on $H$ and assume that the mapping $X: \Omega \rightarrow H$ is Borelmeasurable. Because $H$ is separable, it has a countable orthonormal basis $\left\{\psi_{i}\right\}_{i \in \mathbb{N}}$. We can therefore define the mean of $X$ by

$$
\mathbb{E}(X)=\sum_{i \in \mathbb{N}} \mathbb{E}\left(\left\langle X, \psi_{i}\right\rangle\right) \psi_{i}
$$

which exists in $H$ provided $\mathbb{E}\left(\|X\|_{2}^{2}\right)<\infty$. It is of interest to consider also higher order moments. We shall introduce these as tensors in a tensor product space $H \otimes \cdots \otimes H$ of appropriate dimension. That, is for random elements $X_{1}, \ldots, X_{k}$ in $H$, the moment tensor of order $k$ can be defined as

$$
\mathbb{E}\left(X_{1} \otimes \cdots \otimes X_{k}\right)=\sum_{i_{1}, \ldots i_{k} \in \mathbb{N}} \mathbb{E}\left(\prod_{j=1}^{k}\left\langle X_{j}, \psi_{i_{j}}\right\rangle\right)\left(\psi_{i_{1}} \otimes \cdots \otimes \psi_{i_{k}}\right),
$$

where the elementary tensors $\left\{\psi_{i_{1}} \otimes \cdots \otimes \psi_{i_{k}}\right\}_{1_{1}, \ldots, i_{k} \in \mathbb{N}}$ form an orthonormal basis in $\otimes_{j=1}^{k} H$. The latter follows straightforwardly by the fact that $\left\{\psi_{i}\right\}_{i \in \mathbb{N}}$ is an orthonormal basis of the separable space $H$. Similarly, we can define the $k$-th order cumulant tensor by

$$
\operatorname{cum}\left(X_{1} \otimes \cdots \otimes X_{k}\right)=\sum_{i_{1}, \ldots i_{k} \mathbb{N}} \operatorname{cum}\left(\prod_{j=1}^{k}\left\langle X_{j}, \psi_{i_{j}}\right\rangle\right)\left(\psi_{i_{1}} \otimes \cdots \otimes \psi_{i_{k}}\right)
$$

where the cumulants on the right-hand side are as usual given by

$$
\operatorname{cum}\left(\left\langle X_{1}, \psi_{i_{1}}\right\rangle, \ldots,\left\langle X_{k}, \psi_{i_{k}}\right\rangle\right)=\sum_{\nu=\left(\nu_{1}, \ldots, \nu_{p}\right)}(-1)^{p-1}(p-1) ! \prod_{r=1}^{p} \mathbb{E}\left(\prod_{j \in \nu_{r}}\left\langle X_{j}, \psi_{i_{j}}\right\rangle\right)
$$

where the summation extends over all unordered partitions $\nu$ of $\{1, \ldots, k\}$. 
More generally, we also require the case where the $X_{i}$ are themselves tensors. That is, $X_{i}=\otimes_{j=1}^{J_{i}} X_{i j}, i=1, \ldots, k$, for random elements $X_{i j}$ in $H$ with $j=1, \ldots, J_{i}$ and $i=1, \ldots, k$. To do so, Let $\nu=\left\{\nu_{1}, \ldots \nu_{p}\right\}$ be a partition of $\{1, \ldots, k\}$. Define the probability measure $\mathbb{P}_{\nu}$ on $\left(H \otimes \cdots \otimes H, \mathscr{B}_{H \otimes \cdots \otimes H}\right)$ such that the preimage of the projection mappings $\pi_{v_{l}}{ }^{-1}\left(\bigotimes_{j \in \nu_{l}} X_{j} \in B_{j}\right)$ are independent for all $l=1, \ldots, p$. Then, we can write $\mathbb{P}_{\nu}=\bigotimes_{l=1}^{p} \mathbb{P}_{l}$ with

$$
\mathbb{P}_{\nu}\left(X_{1} \otimes \cdots \otimes X_{p} \in B_{1} \otimes \cdots \otimes B_{p}\right)=\prod_{l=1}^{p} \mathbb{P}\left(\bigotimes_{j \in \nu_{l}} X_{j} \in B_{j}\right) .
$$

The joint cumulant tensor $\operatorname{cum}\left(X_{1}, \ldots, X_{k}\right)$ can then be given by an appropriate generalization of the product theorem for cumulants (Brillinger, 1981, Theorem 2.3.2) to the tensor case,

$$
\operatorname{cum}\left(X_{1}, \ldots, X_{k}\right)=\sum_{r_{11}, \ldots, r_{k J}} \sum_{\nu=\left(\nu_{1}, \ldots, \nu_{p}\right)} \prod_{n=1}^{p} \operatorname{cum}\left(\left\langle X_{i j}, \psi_{r_{i j}}\right\rangle \mid(i, j) \in \nu_{n}\right) \psi_{r_{11}} \otimes \cdots \otimes \psi_{r_{k l_{k}}},
$$

where the summation extends over all indecomposable partitions $\nu=\left(\nu_{1}, \ldots, \nu_{p}\right)$ of the table

$$
\begin{array}{ccc}
(1,1) & \cdots & \left(1, J_{1}\right) \\
\vdots & \ddots & \vdots \\
(k, 1) & \cdots & \left(k, J_{k}\right) .
\end{array}
$$

Formally, we also abbreviate this by

$$
\operatorname{cum}\left(X_{1}, \ldots, X_{k}\right)=\sum_{\nu=\left(\nu_{1}, \ldots, \nu_{p}\right)} S_{\nu}\left(\bigotimes_{n=1}^{p} \operatorname{cum}\left(X_{i j} \mid(i, j) \in \nu_{n}\right)\right),
$$

where $S_{\nu}$ is the permutation that maps the components of the tensor back into the original order, that is, $S_{\nu}\left(\otimes_{r=1}^{p} \otimes_{(i, j) \in \nu_{r}} X_{i j}\right)=X_{11} \otimes \cdots \otimes X_{k J_{k}}$.

\section{A.2.2 Linear transformations of elementary tensors}

As in Appendix A.1, let $A_{1}, \ldots, A_{k}$ be a sequence of bounded linear operators on $H$ let $A_{1} \otimes \cdots \otimes A_{k}$ be the operator on $H \otimes \cdots \otimes H$ given by

$$
\left(A_{1} \otimes \cdots \otimes A_{k}\right)\left(x_{1} \otimes \cdots \otimes x_{k}\right)=\left(A_{1} x_{1}\right) \otimes \cdots \otimes\left(A_{k} x_{k}\right)
$$

for all $x_{1}, \ldots, x_{k} \in H$. The next proposition states that moment tensors - and hence also cumulant tensors by the above definitions - transform linearly.

Proposition A.2.1. Let $A_{1}, \ldots, A_{k}$ be bounded linear operators on $H$ and $X_{1}, \ldots, X_{k}$ be random elements in $H$. Define the mapping $\mathcal{A}=\left(A_{1} \otimes \cdots \otimes A_{k}\right): \otimes_{l=1}^{k} H \rightarrow$ $\bigotimes_{l=1}^{k} H$. Then this map commutes with the moment tensor $\mathbb{E}$, i.e.,

$$
\begin{aligned}
\mathcal{A} \mathbb{E}\left(X_{1} \otimes \cdots \otimes X_{k}\right) & =\mathbb{E} \mathcal{A}\left(X_{1} \otimes \cdots \otimes X_{k}\right) \\
& =\mathbb{E}\left(\left(A_{1} X_{1}\right) \otimes \cdots \otimes\left(A_{k} X_{k}\right)\right) .
\end{aligned}
$$


Proof. Let $\left\{\psi_{i}\right\}_{i \in \mathbb{N}}$ be an orthonormal basis of $H$ and henceforth let $\left\{\boldsymbol{\psi}_{i}\right\}_{i \in \mathbb{N}}=$ $\left\{\psi_{i_{1}} \otimes \cdots \otimes \psi_{i_{k}}\right\}_{i_{1}, \ldots, i_{k} \in \mathbb{N}}$ be an orthonormal basis of $H \otimes \cdots \otimes H$. By definition of the moment tensor, we find

$$
\begin{aligned}
\mathcal{A} \mathbb{E}\left(X_{1} \otimes \cdots \otimes X_{k}\right) & =\mathcal{A} \sum_{i_{1}, \ldots i_{k} \in \mathbb{N}} \mathbb{E}\left(\prod_{j=1}^{k}\left\langle X_{j}, \psi_{i_{j}}\right\rangle\right)\left(\psi_{i_{1}} \otimes \cdots \otimes \psi_{i_{k}}\right) \\
& =\sum_{i_{1}, \ldots i_{k} \in \mathbb{N}} \mathbb{E}\left(\prod_{j=1}^{k}\left\langle X_{j}, \psi_{i_{j}}\right\rangle\right) \mathcal{A}\left(\boldsymbol{\psi}_{i}\right)
\end{aligned}
$$

and by exanding $\mathcal{A}\left(\boldsymbol{\psi}_{i}\right)$ with respect to the chosen orthornormal basis $\left\{\boldsymbol{\psi}_{n}\right\}_{n \in \mathbb{N}}$

$$
\begin{aligned}
& =\sum_{i_{1}, \ldots i_{k} \in \mathbb{N}} \sum_{n \in \mathbb{N}} \mathbb{E}\left(\prod_{j=1}^{k}\left\langle X_{j}, \psi_{i_{j}}\right\rangle\right)\left\langle\mathcal{A}\left(\boldsymbol{\psi}_{i}\right), \boldsymbol{\psi}_{n}\right\rangle \boldsymbol{\psi}_{n} \\
& =\sum_{n \in \mathbb{N}} \mathbb{E}\left[\prod_{j=1}^{k}\left\langle\mathcal{A}\left(\sum_{i_{1}, \ldots i_{k} \in \mathbb{N}}\left\langle X_{j}, \psi_{i_{j}}\right\rangle\right) \boldsymbol{\psi}_{i}, \boldsymbol{\psi}_{n}\right\rangle\right] \boldsymbol{\psi}_{n} \\
& =\sum_{n \in \mathbb{N}} \mathbb{E}\left\langle\mathcal{A}\left(X_{1} \otimes \cdots \otimes X_{k}\right), \boldsymbol{\psi}_{n}\right\rangle \boldsymbol{\psi}_{n} \\
& =\mathbb{E}\left(A_{1} X_{1} \otimes \cdots \otimes A_{k} X_{k}\right),
\end{aligned}
$$

where we used the linearity of the operator $\mathcal{A}$, of the inner product and the ordinary mean.

The cumulant operation can be viewed as a linear combination of expectation operations. The linearity of the cumulant tensors are therefore a direct consequence of the above proposition. That is, we have

Corollary A.2.2. Let $A_{i j} ; j=1, \ldots, J_{i} ; i=1, \ldots, I$ be an array of bounded linear operators on $H$. Then the cumulant tensor defined in 4.24 satisfies

$$
\bigotimes_{i=1}^{I}\left(\bigotimes_{j=1}^{J_{i}} A_{i j}\right) \operatorname{cum}\left(X_{1}, \ldots, X_{I}\right)=\operatorname{cum}\left(\left(\bigotimes_{j=1}^{J_{i}} A_{1 j}\right) X_{1}, \ldots,\left(\bigotimes_{j=1}^{J_{I}} A_{I j}\right) X_{I}\right) \text {. }
$$

Finally, the next theorem formally states the inversion formula for higher order cumulant spectral tensors introduced in Section 2.

Theorem A.2.3 (Inversion formula). Let $\left\{X_{t}\right\}_{t \in \mathbb{Z}}$ be a stationary sequence taking values in $H_{\mathbb{R}}$ such that the cumulant kernel function satisfies

$$
\sum_{t_{1}, \ldots, t_{k-1}=-\infty}^{\infty}\left\|c_{t_{1}, \ldots, t_{k-1}}\right\|_{p}<\infty
$$

for $p=2$ or $p=\infty$. Then, for all $\boldsymbol{\omega}=\left(\omega_{1}, \ldots, \omega_{k}\right) \in \mathbb{R}^{k}$, the Fourier transform of $c_{t_{1}, \ldots, t_{k-1}}$ given by

$$
f_{\omega_{1}, \ldots, \omega_{k}}\left(\tau_{1}, \ldots, \tau_{k}\right)=\frac{1}{(2 \pi)^{k-1}} \sum_{t_{1}, . ., t_{k-1}=-\infty}^{\infty} c_{t_{1}, \ldots, t_{k-1}}\left(\tau_{1}, \ldots \tau_{k}\right) e^{-\mathrm{i} \sum_{j=1}^{k-1} \omega_{j} t_{j}},
$$


converges uniformly in $\boldsymbol{\omega}$ with respect to $\|\cdot\|_{p}$. In the manifold $\sum_{j=1}^{k} \omega_{j} \equiv 0 \bmod 2 \pi$, it is uniformly continuous with respect to the $L^{p}$-norm. The inverse Fourier transform

$$
c_{t_{1}, \ldots, t_{k-1}}\left(\tau_{1}, \ldots \tau_{k}\right)=\int_{\Pi^{k}} e^{\mathrm{i} \sum_{j=1}^{k-1} \alpha_{j} t_{j}} f_{\alpha_{1}, \ldots, \alpha_{k-1}}\left(\tau_{1}, \ldots, \tau_{k}\right) \eta\left(\sum_{j=1}^{k} \alpha_{j}\right) d \alpha_{1} \cdots d \alpha_{k}
$$

holds in $\|\cdot\|_{p}$ for all $\left(t_{1}, \ldots, t_{k-1}\right) \in \mathbb{Z}^{k-1}$ and $\boldsymbol{\tau}=\left(\tau_{1}, \ldots, \tau_{k}\right) \in[0,1]^{k}$.

Proof of Proposition A.2.3. The convergence in $\|\cdot\|_{p}$ of (3.69) follows straightforwardly from the triangle inequality. For the inversion formula, subsitution of (3.70) into (3.69) yields

$$
\begin{aligned}
& \left.\int_{\Pi^{k-1}} e^{\mathrm{i} \sum_{j=1}^{k-1} \alpha_{j} t_{j}} \frac{1}{(2 \pi)^{k-1}} \sum_{s_{1}, \ldots, s_{k-1}=-\infty}^{\infty} c_{s_{1}, \ldots, s_{k-1}}\left(\tau_{1}, \ldots, \tau_{k}\right) e^{-\mathrm{i} \sum_{j=1}^{k-1} \alpha_{j} s_{j}} \alpha_{j}\right) d \alpha_{1} \cdots d \alpha_{k-1} \\
& =\sum_{s_{1}, \ldots, s_{k}=-\infty}^{\infty} \frac{1}{(2 \pi)^{k-1}} c_{s_{1}, \ldots, s_{k-1}}\left(\tau_{1}, \ldots, \tau_{k}\right) \int_{\Pi^{k-1}} e^{\mathrm{i} \sum_{j=1}^{k-1} \alpha_{j}\left(t_{j}-s_{j}\right)} d \alpha_{1} \cdots d \alpha_{k-1} \\
& =c_{t_{1}, \ldots, t_{k-1}}\left(\tau_{1}, \ldots, \tau_{k}\right) .
\end{aligned}
$$

The permutation of the sum and integrals in case $p=\infty$ is justified by the Dominated Convergence Theorem since

$$
\begin{aligned}
& \left|\sum_{s_{1}, \ldots, s_{k-1}=-N}^{N} c_{s_{1}, \ldots, s_{k-1}}\left(\tau_{1}, \ldots, \tau_{k}\right) e^{\mathrm{i} \sum_{j=1}^{k} \alpha_{j}\left(t_{j}-s_{j}\right)}\right| \\
& \leqslant \sup _{\tau} \sum_{s_{1}, \ldots, s_{k-1}=-\infty}^{\infty}\left|c_{s_{1}, \ldots, s_{k-1}}\left(\tau_{1}, \ldots, \tau_{k}\right)\right|<\infty,
\end{aligned}
$$

Consider then the case $p=2$. Because the space $L^{2}$ is isomorphic to the sequence space $\ell^{2}$, it will be sufficient to show equality of the projections

$$
\left\langle\int_{\Pi^{k-1}} e^{\mathrm{i} \sum_{j=1}^{k-1} \alpha_{j} t_{j}} f_{\alpha_{1}, \ldots, \alpha_{k-1}} d \alpha_{1} \cdots d \alpha_{k-1}, \boldsymbol{\psi}_{i}\right\rangle=\left\langle c_{t_{1}, \ldots, t_{k-1}}, \boldsymbol{\psi}_{i}\right\rangle
$$

where $\left\{\boldsymbol{\psi}_{i}\right\}_{i \in \mathbb{N}}=\left\{\psi_{i_{1}} \otimes \cdots \otimes \psi_{i_{k}}\right\}_{i_{1}, \ldots, i_{k} \in \mathbb{N}}$ is an orthonormal basis of $H \otimes \cdots \otimes H$. Using the Cauchy Schwarz Inequality, the right-hand side of (3.70) can be bounded by

$$
\begin{aligned}
\frac{1}{(2 \pi)^{k-1}} \int_{[0,1]^{k}} \mid & \int_{\Pi^{k-1}} e^{\mathrm{i} \sum_{j=1}^{k-1} \alpha_{j}\left(t_{j}-s_{j}\right)} \sum_{s_{1}, \ldots, s_{k-1}=-\infty}^{\infty} c_{t_{1}, \ldots, t_{k-1}}\left(\tau_{1}, \ldots \tau_{k}\right) \\
& \times \psi_{i_{1}}\left(\tau_{1}\right) \cdots \psi_{i_{k}}\left(\tau_{k}\right) d \alpha_{1} \cdots d \alpha_{k-1} \mid d \tau_{1} \cdots d \tau_{k} \\
& \leqslant\left\|\sum_{s_{1}, \ldots, s_{k-1}=-\infty}^{\infty} c_{s_{1}, \ldots, s_{k-1}}\right\|_{2} \prod_{j=1}^{k}\left\|\psi_{i_{j}}\right\|_{2}<\infty .
\end{aligned}
$$

The Dominated Convergence Theorem therefore justifies again the permutation of sums and integrals, which completes the proof. 


\section{A.3 Data taper}

In order to show convergence of the higher order cumulants of the estimator in (3.44), we will make use of two lemmas from Dahlhaus (1993) (Lemma A.4 and A.5 resp.). Both rely on the function $L_{T}: \mathbb{R} \rightarrow \mathbb{R}, T \in \mathbb{R}^{+}$, which is the $2 \pi$-periodic extension of

$$
L_{T}(\lambda)= \begin{cases}T & \text { if }|\lambda| \leqslant 1 / T, \\ 1 /|\lambda| & \text { if } 1 / T \leqslant|\lambda| \leqslant \pi .\end{cases}
$$

The function $L_{T}$ satisfies some nice properties. The following lemma lists those required in the current chapter:

Lemma A.3.1. Let $k, l, T \in \mathbb{N}, \lambda, \alpha, \omega, \mu, \gamma \in \mathbb{R}$ and $\Pi:(-\pi, \pi]$. The following inequalities then hold with a constant $C$ independent of $T$.

(i) $L_{T}(\lambda)$ is monotone increasing in $T$ and decreasing in $\lambda \in[0, \pi]$;

(ii) $|\lambda| L_{T}(\lambda) \leqslant C$ for all $|\lambda| \leqslant \pi$;

(iii) $\int_{\Pi} L_{T}(\lambda) d \lambda \leqslant C \log T$;

(iv) $\int_{\Pi} L_{T}(\lambda)^{k} d \lambda \leqslant C T^{k-1}$ for $k>1$;

(v) $\int_{\Pi} L_{T}(\alpha-\lambda) L_{T}(\lambda+\gamma) d \lambda \leqslant C L_{T}(\alpha+\gamma) \log T$.

In addition, we also make use of Lemma 2 from Eichler (2007).

Lemma A.3.2. Let $\left\{P_{1}, \ldots, P_{m}\right\}$ be an indecomposable partition of the table

$$
\begin{array}{cc}
\alpha_{1} & -\alpha_{1} \\
\vdots & \vdots \\
\alpha_{n} & -\alpha_{n}
\end{array}
$$

with $n \geqslant 3$. For $P_{j}=\left\{\gamma_{j 1}, \ldots, \gamma_{j d_{j}}\right\}$, let $\bar{\gamma}_{j}=\gamma_{j 1}+\ldots+\gamma_{j d_{j}}$.

(i) If $m=n$ then for any $n-2$ variables $\alpha_{i_{1}}, \ldots, \alpha_{i_{n-2}}$ we have

$$
\int_{\Pi^{k-2}} \prod_{j=1}^{n} L_{T}\left(\bar{\gamma}_{j}\right) d \alpha_{i_{1}} \cdots d \alpha_{i_{n-2}} \leqslant C L_{N}\left(\alpha_{i_{n-1}} \pm \alpha_{i_{n}}\right)^{2} \log (T)^{n-2} .
$$

(ii) If $m<n$ then there exists $n-2$ variables $\alpha_{i_{1}}, \ldots, \alpha_{i_{n-2}}$ such that

$$
\int_{\Pi^{k-2}} \prod_{j=1}^{n} L_{T}\left(\bar{\gamma}_{j}\right) d \alpha_{i_{1}} \cdots d \alpha_{i_{n-2}} \leqslant C T \log (T)^{n-2} .
$$

The usefulness of the $L_{T}$ function stems from the fact that it gives an upperbound for the function $H_{k, N}$ which was defined in Section 3.5. Namely, we have

$$
\left|H_{k, N}^{(\lambda)}\right| \leqslant L_{N}(\lambda), \forall k \in \mathbb{N} .
$$

We also require an adjusted version of Lemma A.5 of Dahlhaus (1993): 
Lemma A.3.3. Let $N, T \in \mathbb{N}$. Suppose $h$ is a data taper of bounded variation and let the operator-valued function $G_{u}:[0,1] \rightarrow S_{p}(H)$ be continuously differentiable in $u$ such that $\left\|\frac{\partial G_{u}}{\partial u}\right\|_{p}<\infty$ uniformly in $u$. Then we have for $0 \leqslant t \leqslant N$,

$$
\begin{aligned}
H_{N}\left(G_{\dot{\bar{T}}}, \omega\right) & =H_{N}(\omega) G_{\frac{t}{T}}+O\left(\sup _{u}\left\|\frac{\partial}{\partial u} G_{u}\right\| \|_{p} \frac{N}{T} L_{N}(\omega)\right) \\
& =O\left(\sup _{u \leqslant N / T}\left\|G_{u}\right\|_{p} \frac{N}{T} L_{N}(\omega)+\sup _{u}\left\|\frac{\partial}{\partial u} G_{u}\right\| \|_{p} \frac{N}{T} L_{N}(\omega)\right),
\end{aligned}
$$

where $H_{N}\left(G_{\bullet}, \omega\right)$ is as in (3.41) The same holds if $G_{\dot{\bar{T}}}$ on the left-hand side is replaced by operators $G_{\bullet}^{(T)}$ for which $\sup _{s}\left\|G_{\bullet}^{(T)}-G_{\cdot}\right\|_{p}=O\left(\frac{1}{T}\right)$.

Proof. Summation by parts gives

$$
\begin{aligned}
H_{N}\left(G_{\dot{\bar{T}}}, \omega\right)-H_{N}(\omega) G_{\frac{t}{T}} & =\sum_{s=0}^{N-1}\left[G_{\frac{s}{T}}-G_{\frac{t}{T}}\right] h_{s, N} e^{-\mathrm{i} \omega s} \\
& =-\sum_{s=0}^{N-1}\left[G_{\frac{s}{T}}-G_{\frac{s-1}{T}}\right] H_{s}\left(h_{\bullet, N}, \omega\right)+\left[G_{\frac{N-1}{T}}-G_{\frac{t}{T}}\right] H_{N}(\omega) .
\end{aligned}
$$

It has been shown in Dahlhaus (1988) that $\left|H_{s}\left(h_{\bullet}, N, \omega\right)\right| \leqslant K L_{s}(\omega) \leqslant K L_{N}(\omega)$. The result in 3.73 then follows since

$$
\left\|G_{b}-G_{a}\right\|_{p} \leqslant \sup _{a<\xi<b}\left\|\left.\frac{\partial}{\partial u} G_{u}\right|_{u=\xi}\right\| \|_{p}|b-a|, \quad a, b \mathbb{R},
$$

by the Mean Value Theorem. The lemma holds additionally for operators $G_{\bullet}^{(T)}$ that satisfy $\sup _{s}\left\|G_{\bullet}^{(T)}-G_{\dot{T}}\right\|_{p}=O\left(\frac{1}{T}\right)$. This is a consequence of Minkowski's Inequality since

$$
\begin{aligned}
& \left\|H_{N}\left(G_{\bullet}^{(T)}-G_{\dot{\bar{T}}}, \omega\right)+H_{N}\left(G_{\dot{\bar{T}}}, \omega\right)\right\|_{p} \\
& =\left\|H_{N}\left(G_{\bullet}^{(T)}-G_{\dot{\bar{T}}}, \omega\right)\right\|_{p}+\left\|H_{N}\left(G_{\dot{\bar{T}}}, \omega\right)\right\|_{p} \\
& =O\left(\frac{N}{T}+L_{N}(\lambda)\right)=O\left(L_{N}(\lambda)\right) .
\end{aligned}
$$

Hence, the replacement error is negligible compared to the error of 3.73.

If $p=2$, the above implies that the kernel function $g_{u} \in H_{\mathbb{C}}^{2}$ of $G_{u}$ satisfies

$$
\begin{aligned}
& \left\|H_{N}\left(g_{\dot{\bar{T}}}, \omega\right)-H_{N}(\omega) g_{\frac{t}{T}}\right\|_{2}=R_{1, N}, \\
& \left\|H_{N}\left(g_{\dot{\bar{T}}}, \omega\right)\right\|=R_{2, N}+R_{1, N}
\end{aligned}
$$

where

$$
\begin{aligned}
\left\|R_{1, N}\right\|_{2} & =O\left(\sup _{u}\left\|\frac{\partial}{\partial u} g_{u}\right\|_{p} \frac{N}{T} L_{N}(\omega)\right), \\
\left\|R_{2, N}\right\|_{2} & =O\left(\sup _{u \leqslant N / T}\left\|g_{u}\right\|_{p} \frac{N}{T} L_{N}(\omega) .\right.
\end{aligned}
$$

Similarly if $g_{\dot{T}}$ on the left-hand side is replaced by the kernel function $g_{\bullet}^{(T)} \in H_{\mathbb{C}}^{2}$ of $G_{\bullet}^{(T)}$. If the kernels are bounded uniformly in their functional arguments, Lemma A.5 of Dahlhaus (1993) is point-wise applicable. 


\section{A.4 Proofs}

\section{A.4.1 Proofs of section 3.2}

Proof of Theorem 3.2.2. Let $\mu$ be the measure on the interval $[-\pi, \pi]$ given by

$$
\mu(A)=\int_{A}\left\|\mathcal{F}_{\omega}\right\|_{1} d \omega
$$

for all Borel sets $A \subseteq[-\pi, \pi]$. Similar to the time series setting, it has been shown (Panaretos and Tavakoli, 2013a) that there is a unique isomorphism $\mathcal{T}$ of $\overline{\operatorname{sp}}\left\{X_{t}\right\}_{t \in \mathbb{Z}}$ onto $L_{\mathbb{C}}^{2}([-\pi, \pi], \mu)$ such that

$$
\mathcal{T} X_{t}=e^{\mathrm{i} t}
$$

for all $t \in \mathbb{Z}$. The process defined by $Z_{\omega}=\mathcal{T}^{-1}\left(1_{(-\pi, \omega]}(\cdot)\right)$ is then a functional orthogonal increment process of which the second order properties are completely determined by the spectral density operator $\mathcal{F}$. We have

$$
\mathcal{T}\left(Z_{\omega}-Z_{\nu}\right)=1_{(\nu, \omega]}(\cdot), \quad-\pi<\nu<\omega<\pi,
$$

and for $b_{j} \in \mathbb{C}, j=1, \ldots, N$

$$
\mathcal{T}\left(\sum_{j=1}^{N} b_{j} X_{t_{j}}\right)=\sum_{j=1}^{N} b_{j} e^{\mathrm{i} t_{j}(\cdot)} .
$$

For the first part of the proof, we shall use that the function $1_{(-\pi, \omega]}(\cdot)$ can be approximated by the $N$-th order Fourier series approximation

$$
b_{N}(\lambda)=\sum_{|t| \leqslant N} \tilde{b}_{\omega, t} e^{i t \lambda}
$$

where the Fourier coefficients are given by

$$
\tilde{b}_{\omega, t}=\frac{1}{2 \pi} \int_{-\pi}^{\pi} 1_{(-\pi, \omega]}(\lambda) e^{-i t \lambda} d \lambda
$$

The approximation satisfies the properties listed in the following proposition (Brockwell and Davis, 1991, Proposition 4.11.2).

Proposition A.4.1. Let $\left\{b_{N}\right\}_{N \geqslant 1}$ be the sequence of functions defined in (3.76). Then for $-\pi<\nu<\omega<\pi$,

(i) $\sup _{\lambda \in[-\pi, \pi] \backslash \mathcal{E}}\left|b_{N}(\lambda)-1_{(\nu, \omega]}(\lambda)\right| \rightarrow 0$ as $N \rightarrow \infty$, where $\mathcal{E}$ is an open subset of $[-\pi, \pi]$ containing both $\nu$ and $\omega$;

(ii) $\sup _{\lambda \in[-\pi, \pi]}\left|b_{N}(\lambda)\right| \leqslant C<\infty$ for all $N \geqslant 1$. 
Note then that we can write

$$
Z_{\omega}^{(N)}=\frac{1}{2 \pi} \sum_{|t| \leqslant N} X_{t} \int_{-\pi}^{\pi} 1_{(-\pi, \omega]}(\lambda) e^{\mathrm{i} t \lambda} d \lambda=\sum_{|t| \leqslant N} \tilde{b}_{\omega, t} X_{t},
$$

where $\left\{\tilde{b}_{\omega, t}\right\}_{t \in \mathbb{N}}$ are the Fourier coefficients of the indicator function $1_{(-\pi, \omega]}$. Therefore,

$$
\begin{aligned}
\operatorname{cum} & \left(Z_{\omega_{1}}^{(N)}, \ldots, Z_{\omega_{k}}^{(N)}\right) \\
& =\sum_{\left|t_{1}\right|, \ldots,\left|t_{k}\right| \leqslant N} \tilde{b}_{\omega_{1}, t_{1}} \cdots \tilde{b}_{\omega_{k}, t_{k}} \operatorname{cum}\left(X_{t_{1}} \ldots, X_{t_{k}}\right)
\end{aligned}
$$

and by stationarity of the process $X_{t}$

$$
\begin{aligned}
& =\sum_{\left|t_{1}\right|, \ldots,\left|t_{k}\right| \leqslant N} \tilde{b}_{\omega_{1}, t_{1}} \cdots \tilde{b}_{\omega_{k}, t_{k}} \int_{\Pi^{k}} e^{\mathrm{i}\left(\alpha_{1} t_{1}+\ldots+\alpha_{k} t_{k}\right)} \eta\left(\sum_{j=1}^{k} \alpha_{j}\right) \mathcal{F}_{\alpha_{1} \ldots \alpha_{k-1}} d \alpha_{1} \cdots d \alpha_{k} \\
& =\int_{\Pi^{k}} \eta\left(\sum_{j=1}^{k} \alpha_{j}\right) \mathcal{F}_{\alpha_{1} \ldots \alpha_{k-1}} \prod_{i=1}^{k} \sum_{\left|t_{i}\right|<N}\left(\int_{\Pi^{k}} 1_{\left(-\pi, \omega_{i}\right]}\left(\lambda_{i}\right) e^{-\mathrm{i} t_{i} \lambda_{i}} d \lambda_{i}\right) e^{\mathrm{i} \alpha_{i} t_{i}} d \alpha_{1} \cdots d \alpha_{k} \\
& =\int_{\Pi^{k}} \eta\left(\sum_{j=1}^{k} \alpha_{j}\right) \mathcal{F}_{\alpha_{1} \ldots \alpha_{k-1}} b_{\omega_{1}, N}\left(\alpha_{1}\right) \cdots b_{\omega_{k}, N}\left(\alpha_{k}\right) d \alpha_{1} \cdots d \alpha_{k} .
\end{aligned}
$$

To show convergence, recall that the kernel function $\mathcal{F}_{\alpha_{1} \ldots \alpha_{k-1}}$ is bounded and uniformly continuous in the manifold $\sum_{j=1}^{k} \alpha_{j} \equiv 0 \bmod (2 \pi)$ with respect to $\|\cdot\|_{2}$. An application of Hölder's inequality yields

$$
\begin{aligned}
& \left\|\int_{\Pi^{k}} \eta\left(\sum_{j=1}^{k} \alpha_{j}\right) \mathcal{F}_{\alpha_{1} \ldots \alpha_{k-1}}\left[b_{\omega_{1}, N}\left(\alpha_{1}\right) \cdots b_{\omega_{k}, N}\left(\alpha_{k}\right)-1_{\left(-\pi, \omega_{1}\right]}\left(\alpha_{1}\right) \cdots 1_{\left(-\pi, \omega_{k}\right]}\left(\alpha_{k}\right)\right] d \alpha_{1} \cdots d \alpha_{k}\right\|_{2} \\
& \quad \leqslant \sup _{\alpha_{1}, \ldots, \alpha_{k-1}}\left\|\mathcal{F}_{\alpha_{1} \ldots \alpha_{k-1}}\right\|_{2} \int_{\Pi^{k}}\left|b_{\omega_{1}, N}\left(\alpha_{1}\right) \cdots b_{\omega_{k}, N}\left(\alpha_{k}\right)-1_{\left(-\pi, \omega_{1}\right]}\left(\alpha_{1}\right) \cdots 1_{\left(-\pi, \omega_{k}\right]}\left(\alpha_{k}\right)\right| d \alpha_{1} \cdots d \alpha_{k}
\end{aligned}
$$

A standard telescoping argument together with Proposition A.4.1 gives

$$
\begin{aligned}
& \leqslant K \int_{\Pi^{k}} \sum_{j=1}^{k} \prod_{l=1}^{j-1}\left|b_{\omega_{l}, N}\left(\alpha_{l}\right)\right| \prod_{l=j+1}^{k}\left|1_{\left(-\pi, \omega_{1}\right]}\left(\alpha_{l}\right)\right|\left|b_{\omega_{j}, N}\left(\alpha_{j}\right)-1_{\left(-\pi, \omega_{j}\right]}\left(\alpha_{j}\right)\right| d \alpha_{1} \cdots d \alpha_{k} \\
& \leqslant K k\left(\sup _{1 \leqslant j \leqslant k} \sup _{\alpha}\left|b_{\omega_{j}, N}(\alpha)\right|\right)^{k-1} \sup _{\omega} \int_{\Pi}\left|b_{\omega, N}(\alpha)-1_{(-\pi, \omega]}(\alpha)\right| d \alpha \rightarrow 0
\end{aligned}
$$

as $N \rightarrow \infty$. Hence, the dominated convergence theorem implies

$$
\begin{aligned}
& \lim _{N \rightarrow \infty} \operatorname{cum}\left(Z_{\omega_{1}}^{(N)}, \ldots, Z_{\omega_{k}}^{(N)}\right) \\
& =\frac{1}{(2 \pi)^{k}} \int_{\Pi^{k}} 1_{\left(-\pi, \omega_{1}\right]}\left(\alpha_{1}\right) \cdots 1_{\left(-\pi, \omega_{k}\right]}\left(\alpha_{k}\right) \mathcal{F}_{\alpha_{1} \ldots \alpha_{k-1}} \eta\left(\sum_{j=1}^{k} \alpha_{j}\right) d \alpha_{1} \cdots d \alpha_{k} \\
& =\frac{1}{(2 \pi)^{k}} \int_{-\pi}^{\omega_{1}} \cdots \int_{-\pi}^{\omega_{k}} \eta\left(\sum_{j=1}^{k} \lambda_{j}\right) \mathcal{F}_{\alpha_{1} \ldots \alpha_{k-1}} d \lambda_{1} \cdots d \lambda_{k}, \\
& =\operatorname{cum}\left(Z_{\omega_{1}}, \ldots, Z_{\omega_{k}}\right)
\end{aligned}
$$


which establishes the $L^{2}$ convergence in (3.7). The almost everywhere convergence is proved similarly by replacing $\mathcal{F}$ by $f\left(\tau_{1}, \ldots, \tau_{k}\right)$. In order to show that $X_{t}=$ $\int_{-\pi}^{\pi} e^{\mathrm{i} \omega t} d Z_{\omega}$ with probability 1 , it remains to show that

$$
\mathbb{E}\left\|X_{t}-\int_{-\pi}^{\pi} e^{\mathrm{i} \omega t} d Z_{\omega}\right\|_{2}^{2}=0
$$

We refer to Panaretos and Tavakoli (2013a) for a proof.

Finally, to suggested differential notation follows since

$$
\operatorname{cum}\left(Z_{\omega_{1}}, \ldots, Z_{\omega_{k}}\right)=\int_{-\pi}^{\omega_{1}} \cdots \int_{-\pi}^{\omega_{k}} \eta\left(\sum_{j=1}^{k} \lambda_{j}\right) \mathcal{F}_{\lambda_{1}, \ldots, \lambda_{k-1}} d \lambda_{1} \cdots d \lambda_{k}
$$

we can view $\operatorname{cum}\left(Z_{\omega_{1}}, \ldots, Z_{\omega_{k}}\right)$ as a measure on $[-\pi, \pi]^{k}$ and write

$$
\operatorname{dcum}\left(Z_{\lambda_{1}}, \ldots, Z_{\lambda_{k}}\right)=\eta\left(\sum_{j=1}^{k} \lambda_{j}\right) \mathcal{F}_{\lambda_{1}, \ldots, \lambda_{k-1}} d \lambda_{1} \cdots d \lambda_{k}
$$

By a similar argument as above, we have the projections satisfy

$$
\begin{aligned}
& \lim _{h \downarrow 0}\left\langle\operatorname{cum}\left(Z_{\omega_{1}+h}-Z_{\omega_{1}}, \ldots, Z_{\omega_{k}+h}-Z_{\omega_{k}}\right), \psi_{i_{1}} \otimes \cdots \otimes \psi_{i_{k}}\right\rangle \\
& =\left\langle\lim _{h \downarrow 0} \int_{\omega_{1}}^{\omega_{1}+h} \cdots \int_{\omega_{k}}^{\omega_{k}+h} \eta\left(\sum_{j=1}^{k} \lambda_{j}\right) f_{\lambda_{1}, \ldots, \lambda_{k-1}} d \lambda_{1} \cdots d \lambda_{k}, \psi_{i_{1}} \otimes \cdots \otimes \psi_{i_{k}}\right\rangle \\
& =\left\langle\eta\left(\sum_{j=1}^{k} \omega_{j}\right) f_{\omega_{1}, \ldots, \omega_{k-1}} d \omega_{1} \cdots d \omega_{k}, \psi_{i_{1}} \otimes \cdots \otimes \psi_{i_{k}}\right\rangle=0 .
\end{aligned}
$$

Without loss of generality we can therefore write in differential notation

$$
\operatorname{cum}\left(d Z_{\omega_{1}}\left(\tau_{1}\right), \ldots, d Z_{\omega_{k}}\left(\tau_{k}\right)\right)=\eta\left(\omega_{1}+\ldots+\omega_{k}\right) f_{\omega_{1}, \ldots, \omega_{k-1}}\left(\tau_{1}, \ldots, \tau_{k}\right) d \omega_{1} \cdots d \omega_{k} .
$$

\section{A.4.2 Proofs section 3.3}

Proposition A.4.2. Let $\left\{\varepsilon_{t}\right\}_{t \in \mathbb{Z}}$ be a functional i.i.d. process in $H_{\mathbb{R}}$ with $\mathbb{E}\left\|\varepsilon_{0}\right\|_{2}^{k}<$ $\infty, k \in \mathbb{N}$ and let $\left\{A_{t, s}^{(T)}\right\}_{s \in \mathbb{Z}}$ be a sequence of operators in $S_{\infty}(H)$ satisfying $\sum_{s}\left\|A_{t, s}^{(T)}\right\|_{\infty}<$ $\infty$ for all $t=1, \ldots, T$ and $T \in \mathbb{N}$. Then the process $X_{t, T}^{(N)}=\sum_{|s| \leqslant N} A_{t, s}^{(T)} \varepsilon_{t-s}$ has the following properties:

(i) $X_{t, T}^{(N)} \stackrel{L^{k}(\mathbb{P})}{\longrightarrow} X_{t, T}: X_{t, T}^{(N)}$ converges to a process $X_{t, T} \in L^{k}(\mathbb{P})$.

(ii) $\operatorname{cum}\left(X_{t_{1}, T}, \ldots, X_{t_{k}, T}\right)=\left(\sum_{s_{1} \in \mathbb{Z}} A_{t_{1}, s_{1}}^{(T)} \otimes \cdots \otimes \sum_{s_{k} \in \mathbb{Z}} A_{t_{k}, s_{k}}^{(T)}\right) \operatorname{cum}\left(\varepsilon_{t_{1}-s_{1}}, \ldots, \varepsilon_{t_{k}-s_{k}}\right)$,

where the convergence is with respect to $\|\cdot\|_{2}$. 
Proof of Proposition A.4.2. For the first equality, we need to show that

$$
\lim _{N \rightarrow \infty} \mathbb{E}\left\|X_{t, T}^{(N)}-X_{t, T}\right\|_{2}^{k}=0
$$

We will do this by demonstrating that the the tail series $X_{t, T}^{-(N)}=\sum_{s=N+1}^{M} A_{t, s}^{(T)} \varepsilon_{t-s}$ converges. Since $\left\|A_{t, s}^{(T)} \varepsilon_{t}\right\|_{2} \leqslant\left\|A_{t, s}^{(T)}\right\|\left\|_{\infty}\right\| \varepsilon_{t} \|_{2}$, an application of the generalized Hölder's Inequality yields

$$
\begin{aligned}
\mathbb{E}\left\|X_{t_{1}, T}^{-(N)}\right\|_{2}^{k} & \leqslant \sum_{s_{1}, \ldots, s_{k}=N+1}^{M}\left\|A_{t_{1}, s_{1}}^{(T)}\right\|_{\infty} \cdots\left\|A_{t_{k}, s_{k}}^{(T)}\right\| \|_{\infty} \mathbb{E}\left[\left\|\varepsilon_{t_{1}-s_{1}}\right\|_{2} \cdots\left\|\varepsilon_{t_{k}-s_{k}}\right\|_{2}\right] \\
& \leqslant \sum_{\left|s_{1}\right|, \ldots,\left|s_{k}\right|>N}\left\|A_{t_{1}, s_{1}}^{(T)}\right\|_{\infty} \cdots\left\|A_{t_{k}, s_{k}}^{(T)}\right\|_{\infty}\left[\mathbb{E}\left\|\varepsilon_{t_{1}-s_{1}}\right\|_{2}^{k} \cdots \mathbb{E}\left\|\varepsilon_{t_{k}-s_{k}}\right\|_{2}^{k}\right]^{1 / k} \\
& \leqslant\left(\sum_{|s|>N}\left\|A_{t, s}^{(T)}\right\|_{\infty}\right)^{k} \mathbb{E}\left\|_{\varepsilon_{0}}\right\|_{2}^{k}<\infty
\end{aligned}
$$

uniformly in $M$. Hence, $\lim _{N \rightarrow \infty}\left(\mathbb{E}\left\|X_{t, T}^{-(N)}\right\|_{2}^{k}\right)^{1 / k} \rightarrow 0$.

We now prove 2. By Corollary A.2.2 and 1, we have

$$
\operatorname{cum}\left(A_{t_{1}, s_{1}}^{(T)} \varepsilon_{t_{1}-s_{1}}, \ldots, A_{t_{k}, s_{k}}^{(T)} \varepsilon_{t_{k}-s_{k}}\right)=\left(A_{t_{1}, s_{1}}^{(T)} \otimes \cdots \otimes A_{t_{k}, s_{k}}^{(T)}\right) \operatorname{cum}\left(\varepsilon_{t_{1}-s_{1}}, \ldots, \varepsilon_{t_{k}-s_{k}}\right) .
$$

It is therefore sufficient to show that

$$
\operatorname{cum}\left(\sum_{s_{1} \in \mathbb{Z}} A_{t_{1}, s_{1}}^{(T)} \varepsilon_{t_{1}-s_{1}}, \ldots, \sum_{s_{k} \in \mathbb{Z}} A_{t_{k}, s_{k}}^{(T)} \varepsilon_{t_{k}-s_{k}}\right)=\sum_{s_{1}, \ldots, s_{k} \in \mathbb{Z}} \operatorname{cum}\left(A_{t_{1}, s_{1}}^{(T)} \varepsilon_{t_{1}-s_{1}}, \ldots, A_{s_{k}, t_{k}, T} \varepsilon_{t_{k}-s_{k}}\right) .
$$

Let $\left\{\psi_{l}\right\}_{l \in \mathbb{N}}$ be an orthonormal basis of $H$. Then $\left\{\psi_{l_{1}} \otimes \cdots \otimes \psi_{l_{k}}\right\}_{l_{1}, \ldots, l_{k} \geqslant 1}$ forms an orthonormal basis $\otimes_{j=1}^{k} H$. For the partial sums

$$
\sum_{s_{j}=1}^{N} A_{t_{j}, s_{j}}^{(T)} \varepsilon_{t_{j}-s_{j}}, \quad j=1, \cdots, k
$$

we obtain by virtue of the triangle inequality, the Cauchy-Schwarz Inequality and generalized Hölder Inequality

$$
\begin{aligned}
& \mathbb{E}\left\|\prod_{j=1}^{k} \sum_{s_{j}=1}^{N} A_{t_{j}, s_{j}}^{(T)} \varepsilon_{t_{j}-s_{j}} \psi_{l}\right\|_{1} \leqslant \prod_{j=1}^{k} \mathbb{E}\left\|\sum_{s_{j}=1}^{N} A_{t_{j}, s_{j}}^{(T)} \varepsilon_{t_{j}-s_{j}} \psi_{l}\right\|_{1} \\
& \left(\sup _{t, T} \sum_{s \in \mathbb{Z}}\left\|A_{t, s}^{(T)}\right\|_{\infty}\right)^{k} \mathbb{E}\left\|\varepsilon_{0}\right\|_{2}^{k}<\infty .
\end{aligned}
$$

The result now follows by the Dominated Convergence Theorem. 
Proof of Proposition 3.3.1. For fixed $t$ and $T$, we have by Minkowski's Inequality

$$
\begin{aligned}
& \sum_{s}\left\|\operatorname{cum}\left(X_{\lfloor u T-s / 2\rfloor, T}, X_{\lfloor u T+s / 2\rfloor, T}\right)\right\|_{2} \\
& =\sum_{s}\left\|\frac{1}{2 \pi} \int_{\Pi}\left(\mathcal{A}_{\lfloor u T-s / 2\rfloor, \lambda_{1}}^{(T)} \otimes \mathcal{A}_{\lfloor u T+s / 2\rfloor,-\lambda_{1}}^{(T)}\right) \mathcal{F}_{\lambda_{1}}^{\varepsilon} e^{\mathrm{i} \lambda_{1} s} d \lambda_{1}\right\|_{2} \\
& =\sum_{\substack{s:\{(1 \leqslant\lfloor u T-s / 2\rfloor \leqslant T) \cup \\
(1 \leqslant\lfloor u T+s / 2\rfloor \leqslant T)\}}}\left\|\mathcal{C}_{u, s}^{(T)}\right\|\left\|_{2}+\sum_{\substack{s:\{(1 \leqslant\lfloor u T-s / 2\rfloor \leqslant T) \cup \\
(1 \leqslant\lfloor u T+s / 2\rfloor \leqslant T)\}^{\mathrm{C}}}}\right\| \mathcal{C}_{u, s}^{(T)} \|_{2}
\end{aligned}
$$

where $\{\cdot\}^{\complement}$ denotes the complement event. Now since $\mathcal{A}_{t, \omega}^{(T)}=\mathcal{A}_{0, \omega}$ for $t<1$ and $\mathcal{A}_{t, \omega}^{(T)}=\mathcal{A}_{0, \omega}$ for $t>T$, we can write

$$
\begin{aligned}
& =\sum_{s: B}\left\|\frac{1}{2 \pi} \int_{\Pi}\left(\mathcal{A}_{\lfloor u T-s / 2\rfloor, \lambda_{1}}^{(T)} \otimes \mathcal{A}_{\lfloor u T+s / 2\rfloor,-\lambda_{1}}^{(T)}\right) \mathcal{F}_{\lambda_{1}}^{\varepsilon} e^{\mathrm{i} \lambda_{1} s} d \lambda_{1}\right\|_{2} \\
& +\sum_{s: B^{\complement}}\left\|\frac{1}{2 \pi} \int_{\Pi}\left(\mathcal{A}_{0, \lambda_{1}} \otimes \mathcal{A}_{1,-\lambda_{1}}\right) \mathcal{F}_{\lambda_{1}}^{\varepsilon} e^{\mathrm{i} \lambda_{1} s} d \lambda_{1}\right\|_{2},
\end{aligned}
$$

where $B=\{(1 \leqslant\lfloor u T-s / 2\rfloor \leqslant T) \cup(1 \leqslant\lfloor u T+s / 2\rfloor \leqslant T)\}$. Because the first sum is finite, an application of proposition A.1.3 implies it can be bounded by

$$
K \sup _{t, T, \omega}\left\|\mathcal{A}_{t, \omega}^{(T)}\right\|\left\|_{\infty}^{2}\right\| \mathcal{F}^{\varepsilon} \|_{2}<\infty
$$

for some constant $K$. For the second term, we note that

$$
\frac{1}{2 \pi} \int_{\Pi}\left(\mathcal{A}_{0, \lambda_{1}} \otimes \mathcal{A}_{1,-\lambda_{1}}\right) \mathcal{F}_{\lambda_{1}}^{\varepsilon} e^{\mathrm{i} \lambda_{1} s} d \lambda_{1}=\operatorname{cum}\left(X_{t+s}^{(0)}, X_{t}^{(1)}\right) .
$$

It thus corresponds to the cross-covariance operator of the two stationary processes $X_{t}^{(0)}$ and $X_{t}^{(1)}$ at lag $s$, which we can alternatively express as

$$
\operatorname{cum}\left(X_{s}^{(0)}, X_{0}^{(1)}\right)=\sum_{l, k}\left(A_{0, l} \otimes A_{1, k}\right) \operatorname{cum}\left(\varepsilon_{t+s-l}, \varepsilon_{t-k}\right)
$$

Using then that $\varepsilon_{t}$ is functional white noise, we find for the second term in 3.79

$$
\begin{aligned}
\sum_{s: B^{C}}\left\|\mathcal{C}_{u, s}^{(T)}\right\|_{2} & \leqslant\left\|\sum_{l, k \in \mathbb{Z}}\left(A_{0, l} \otimes A_{1, k}\right) \operatorname{cum}\left(\varepsilon_{0}, \varepsilon_{0}\right)\right\|_{2} \\
& \leqslant \sum_{l \in \mathbb{Z}}\left\|A_{0, l}\right\|_{\infty} \sum_{k \in \mathbb{Z}}\left\|A_{1, k}\right\|\left\|_{\infty}\right\| \operatorname{cum}\left(\varepsilon_{0}, \varepsilon_{0}\right) \|_{2}<\infty
\end{aligned}
$$

The result now follows. 


\section{A.4.3 Proofs of Section 3.5}

Proof of Proposition 3.5.3. We have by Theorem 3.2.2 and by Corollary A.2.2,

$$
\begin{aligned}
& \operatorname{cum}\left(\int_{\Pi} e^{\mathrm{i} \lambda_{1} r_{1}} A_{t_{r_{1}}, \lambda_{1}}^{(T)} d Z_{\lambda_{1}}, \ldots, \int_{\Pi} e^{\mathrm{i} \lambda_{k} r_{k}} A_{t_{r_{k}}, \lambda_{k}}^{(T)} d Z_{\lambda_{k}}\right) \\
& =\int_{\Pi} \cdots \int_{\Pi} \operatorname{cum}\left(e^{\mathrm{i} \lambda_{1} r_{1}} A_{t_{r_{1}}, \lambda_{1}}^{(T)} d Z_{\lambda_{1}}, \ldots, e^{\mathrm{i} \lambda_{k} r_{k}} A_{t_{r_{k}}, \lambda_{k}}^{(T)} d Z_{\lambda_{k}}\right) \\
& =\int_{\Pi} \cdots \int_{\Pi}\left(e^{\mathrm{i} \lambda_{1} r_{1}} A_{t_{r_{1}}, \lambda_{1}}^{(T)} \otimes \cdots \otimes e^{\mathrm{i} \lambda_{k} r_{k}} A_{t_{r_{k}}, \lambda_{k}}^{(T)}\right) \operatorname{cum}\left(d Z_{\lambda_{1}}, \ldots, d Z_{\lambda_{k}}\right) \\
& =\int_{\Pi^{k}} e^{\mathrm{i}\left(\lambda_{1} r_{1}+\ldots+\lambda_{k} r_{k}\right)}\left(\mathcal{A}_{t_{r_{1}}, \lambda_{1}}^{(T)} \otimes \cdots \otimes \mathcal{A}_{t_{r_{k}}, \lambda_{k}}^{(T)}\right) \eta\left(\lambda_{1}+\ldots+\lambda_{k}\right) \mathcal{F}_{\lambda_{1}, \ldots, \lambda_{k-1}}^{\varepsilon} d \lambda_{1} \cdots d \lambda_{k},
\end{aligned}
$$

where the equality holds in the tensor product space $H \otimes \cdots \otimes H$. Note that the last line corresponds to the inversion formula of the cumulant tensor of order $k$. For fixed $t \in\{1, \ldots, T\}$ and $T \in \mathbb{N}$, the $k$-th order cumulant spectral tensor of the linear functional process $\left\{X_{t, T}\right\}$ can thus be given by

$$
\mathcal{F}_{\lambda_{1}, \ldots, \lambda_{k-1}}^{(t, T)}=\left(\mathcal{A}_{t_{r_{1}}, \lambda_{1}}^{(T)} \otimes \cdots \otimes \mathcal{A}_{t_{r_{k-1}}, \alpha_{k-1}}^{(T)} \otimes \mathcal{A}_{t_{r_{k}},-\lambda_{+}}^{(T)}\right) \mathcal{F}_{\lambda_{1}, \ldots, \lambda_{k-1}}^{\varepsilon},
$$

and is well-defined in the tensor product space $\bigotimes_{i=1}^{k} H$. In particular, Proposition A.1.3 implies the corresponding operator is Hilbert-Schmidt for $k \geqslant 2$

$$
\begin{aligned}
& \left\|\mathcal{F}_{\lambda_{1}, \ldots, \lambda_{2 k-1}}\right\|_{2} \leqslant\left\|\mathcal{A}_{t_{r_{1}}, \lambda_{1}}^{(T)} \otimes \cdots \otimes \mathcal{A}_{t_{r_{k-1}}, \alpha_{k-1}}^{(T)} \otimes \mathcal{A}_{t_{r_{k}},-\lambda_{+}}^{(T)}\right\|\left\|_{\infty}\right\| \mathcal{F}_{\lambda_{1}, \ldots, \lambda_{2 k-1}}^{\varepsilon} \|_{2} \\
& =\left(\sup _{t_{r_{j}, \lambda_{j}}}\left\|\mathcal{A}_{t_{r_{j}}, \lambda_{j}}^{(T)}\right\|_{\infty}\right)^{2 k}\left\|\mathcal{F}_{\lambda_{1}, \ldots, \lambda_{2 k-1}}^{\varepsilon}\right\|_{2}<\infty .
\end{aligned}
$$

We therefore have that the kernel function $f_{\lambda_{1}, \ldots, \lambda_{k-1}}^{(t, T)}\left(\tau_{1}, \ldots, \tau_{k}\right)$ is a properly defined element in $L^{2}\left([0,1]^{2}, \mathbb{C}\right)$. In case $k=2$, we moreover have that $\mathcal{F}_{\lambda_{1}} \in$ $S_{1}(H)$. This follows by the fact that the $\varepsilon_{t}$ are white noise and thus $\left\|\mathcal{F}_{\lambda_{1}}^{\varepsilon}\right\|_{1} \leqslant$ $\sum_{t}\left\|\operatorname{cum}\left(\varepsilon_{t}, \varepsilon_{0}\right)\right\|_{1}=\left\|\mathcal{C}_{0}^{\varepsilon}\right\|_{1}=\mathbb{E}\left\|\varepsilon_{0}\right\|_{2}^{2}<\infty$.

Proof of Theorem 3.5.4. Under Proposition 3.2.4 we have for all $t=1, . ., T$ and $T \in \mathbb{N}$ that $X_{t, T}$ are random elements in $H_{\mathbb{R}}$ and hence by Proposition 3.5.3 and (3.40),

$$
\begin{aligned}
\mathbb{E}\left(I_{u_{j}, \omega}^{(T)}\right) & =\frac{1}{2 \pi H_{2, N}(0)} \operatorname{cum}\left(D_{u_{j}, \omega}^{(T)}, D_{u_{j},-\omega}^{(T)}\right) \\
& =\frac{1}{2 \pi H_{2, N}(0)} \int_{\Pi}\left(H_{N}\left(\mathcal{A}_{t_{j, \bullet}, \lambda}^{(T)}, \omega-\lambda\right) \otimes H_{N}\left(\mathcal{A}_{t_{j, \bullet},-\lambda}^{(T)}, \lambda-\omega\right)\right) \mathcal{F}_{\lambda}^{\varepsilon} d \lambda .
\end{aligned}
$$

In order to replace the transfer function kernels with their continuous approximations, we write

$$
\begin{aligned}
\mathcal{A}_{t_{j, r}, \lambda}^{(T)} & \otimes \mathcal{A}_{t_{j, s},-\lambda}^{(T)}-\mathcal{A}_{u_{j, r}, \omega} \otimes \mathcal{A}_{u_{j, s},-\omega} \\
& =\left(\mathcal{A}_{t_{j, r}, \lambda}^{(T)}-\mathcal{A}_{u_{j, r}, \omega}\right) \otimes \mathcal{A}_{t_{j, s},-\lambda}^{(T)}+\mathcal{A}_{u_{j, r}, \omega} \otimes\left(\mathcal{A}_{t_{j, s},-\lambda}^{(T)}-\mathcal{A}_{u_{j, s},-\omega}\right) .
\end{aligned}
$$


We focus on finding a bound on the first term as the second term can be bounded similarly. Since $H_{N}(\cdot, \cdot)$ is linear in its first argument, we have by the triangle inequality

$$
\begin{aligned}
& \left\|H_{N}\left(\mathcal{A}_{t_{j, r}, \lambda}^{(T)}-\mathcal{A}_{u_{j, r}, \omega}, \omega-\lambda\right)\right\|_{\infty} \\
& \quad \leqslant\left\|H_{N}\left(\mathcal{A}_{t_{j, \bullet}, \lambda}^{(T)}-\mathcal{A}_{u_{j, \bullet}, \lambda}, \omega-\lambda\right)\right\|_{\infty}+\left\|H_{N}\left(\mathcal{A}_{u_{j, \bullet}, \lambda}-\mathcal{A}_{u_{j, \bullet}, \omega}, \omega-\lambda\right)\right\|_{\infty} .
\end{aligned}
$$

For the first term of this expression, condition (ii) of Proposition 3.2.4 and Lemma A.3.3 imply

$$
\left\|\sum_{r=0}^{N-1} h_{r, N}\left(\mathcal{A}_{t_{j, r}, \lambda}^{(T)}-\mathcal{A}_{u_{j, r}, \lambda}\right) e^{-\mathrm{i} r(\omega-\lambda)}\right\|_{\infty} \leqslant C \frac{N}{T}
$$

for some generic constant $C$ independent of $T$. Next, we consider the second term. Similarly as in the proof of Lemma A.3.3, we have

$$
\begin{aligned}
& H_{N}\left(\mathcal{A}_{u_{j, \bullet}, \lambda}-\mathcal{A}_{u_{j, \bullet}, \omega}, \omega-\lambda\right) \\
& =H_{N}(\omega-\lambda)\left(\mathcal{A}_{u_{j}, \lambda}-\mathcal{A}_{u_{j}, \omega}\right)+H_{N}(\omega-\lambda)\left(\mathcal{A}_{u_{j, N-1}, \lambda}-\mathcal{A}_{u_{j, N-1}, \omega}\right) \\
& \quad-\sum_{r=0}^{N-1}\left[\left(\mathcal{A}_{u_{j, r}, \lambda}-\mathcal{A}_{u_{j, r-1}, \lambda}\right)-\left(\mathcal{A}_{u_{j, r}, \omega}-\mathcal{A}_{u_{j, r-1}, \omega}\right)\right] H_{s}(\omega-\lambda) .
\end{aligned}
$$

Since the transfer function operator is twice continuously differentiable in $u$ and $\omega$, we find by two applications of the mean value theorem

$$
\left\|\left(\mathcal{A}_{u_{j, r}, \lambda}-\mathcal{A}_{u_{j, r-1}, \lambda}\right)-\left(\mathcal{A}_{u_{j, r}, \omega}-\mathcal{A}_{u_{j, r-1}, \omega}\right)\right\|_{\infty} \leqslant \sup _{u \in[0,1], \omega \in \Pi}\left\|\frac{\partial^{2} \mathcal{A}_{u, \omega}}{\partial u \partial \omega}\right\| \frac{|\lambda-\omega|}{T} .
$$

Hence we obtain the upper bound

$$
\left\|H_{N}\left(\mathcal{A}_{u_{j, \boldsymbol{\bullet}}, \lambda}-\mathcal{A}_{u_{j, \boldsymbol{\bullet}}, \omega}, \omega-\lambda\right)\right\|_{\infty} \leqslant C L_{N}(\omega-\lambda)|\omega-\lambda|+C \frac{N}{T} L_{N}(\omega-\lambda)|\omega-\lambda| .
$$

Moreover, Lemma A.3.3 implies

$$
\left\|H_{N}\left(\mathcal{A}_{t_{j, \bullet},-\lambda}^{(T)}, \omega-\lambda\right)\right\|_{\infty} \leqslant C L_{N}(\omega-\lambda) .
$$

With these bounds and Proposition A.1.3 and Lemma A.3.1, we now obtain

$$
\begin{aligned}
& \int_{\Pi}\left\|\left(H_{N}\left(\mathcal{A}_{u_{j, \boldsymbol{\bullet}}, \lambda}-\mathcal{A}_{u_{j, \boldsymbol{\bullet}}, \omega}, \omega-\lambda\right) \otimes H_{N}\left(\mathcal{A}_{t_{j, \bullet},-\lambda}^{(T)}, \omega-\lambda\right)\right) \mathcal{F}_{\lambda}^{\varepsilon}\right\|_{2} d \lambda \\
& \quad \leqslant \int_{\Pi}\left\|H_{N}\left(\mathcal{A}_{u_{j, \bullet}, \lambda}-\mathcal{A}_{u_{j, \bullet}, \omega}, \omega-\lambda\right)\right\|_{\infty}\left\|H_{N}\left(\mathcal{A}_{t_{j, \bullet},-\lambda}^{(T)}, \omega-\lambda\right)\right\|\left\|_{\infty}\right\| \mathcal{F}_{\lambda}^{\varepsilon} \|_{2} d \lambda \\
& \quad \leqslant C \int_{\Pi} L_{N}(\omega-\lambda)^{2} d \lambda \leqslant C \log (N) .
\end{aligned}
$$

The second term of (3.81) is similar and thus the error from replacing $\mathcal{A}_{t_{j, r}, \lambda}^{(T)}$ and $\mathcal{A}_{t_{j, s},-\lambda}^{(T)}$ by $\mathcal{A}_{u_{j, r}, \omega}$ and $\mathcal{A}_{u_{j, s},-\omega}$, respectively, is of order $O\left(\frac{\log (N)}{N}\right)$ in $L^{2}$. 
The expectation of the periodogram tensor can therefore be written as

$$
\begin{aligned}
\mathbb{E}\left(I_{u_{j}, \omega}^{(T)}\right) & =\frac{1}{2 \pi H_{2, N}(0)} \int_{\Pi}\left(H_{N}\left(\mathcal{A}_{t_{j, \bullet}, \lambda}^{(T)}, \omega-\lambda\right) \otimes H_{N}\left(\mathcal{A}_{t_{j, \bullet},-\lambda}^{(T)}, \lambda-\omega\right)\right) \mathcal{F}_{\lambda}^{\varepsilon} d \lambda \\
& =\frac{1}{2 \pi H_{2, N}(0)} \int_{\Pi}\left(H_{N}\left(\mathcal{A}_{u_{j, \boldsymbol{\bullet}}, \omega}, \omega-\lambda\right) \otimes H_{N}\left(\mathcal{A}_{u_{j, \bullet},-\omega}, \lambda-\omega\right)\right) \mathcal{F}_{\lambda}^{\varepsilon} d \lambda+R_{T} \\
& =\frac{1}{H_{2, N}(0)} H_{2, N}\left(\mathcal{A}_{u_{j, \bullet}, \omega} \otimes \mathcal{A}_{u_{j, \bullet},-\omega}, 0\right) \mathcal{F}_{\omega}^{\varepsilon}+R_{T} \\
& =\frac{1}{H_{2, N}(0)} H_{2, N}\left(\mathcal{F}_{u_{j, \bullet}, \omega}, 0\right)+R_{T}
\end{aligned}
$$

where the remainder term $R_{T}$ is of order $O\left(\frac{\log (N)}{N}\right)$. Correspondingly, the local periodogram kernel is given by

$$
\mathbb{E}\left(I_{u_{j}, \omega}^{(T)}(\tau, \sigma)=\frac{1}{H_{2, N}(0)} \sum_{r=1}^{N} h_{r, N}^{2} f_{u_{j, r}, \omega}(\tau, \sigma)+O\left(\frac{\log (N)}{N}\right) .\right.
$$

Since by the conditions of the theorem, the operator-valued function $\mathcal{A}_{u, \omega}$ is twice continuously differentiable with respect to $u$, Theorem A.1.8 implies that the spectral density tensor $\mathcal{F}_{u, \omega}(\tau, \sigma)$ is also twice continuously differentiable in $u \in(0,1)$. Hence, by a Taylor approximation of $\mathcal{F}_{u_{j, r}, \omega}$ about $u_{j}$, we find for the mean of the periodogram tensor

$$
\mathbb{E}\left(I_{u_{j}, \omega}^{(T)}\right)=\mathcal{F}_{u_{j}, \omega}+\left.b_{\mathrm{t}, T}^{2} \kappa_{\mathrm{t}} \frac{\partial^{2} \mathcal{F}_{u, \omega}}{\partial u^{2}}\right|_{u=u_{j}}+O\left(\frac{\log (N)}{N}\right)+O\left(\frac{N}{T}\right)
$$

where we have used the definition in (3.43) of the smoothing kernel $K_{\mathrm{t}}$ in time direction. As by the assumption on the taper function this kernel is symmetric about zero, the first order term in the Taylor approximation is zero.

This proves the first part of from Theorem 3.5.4. For the covariance, we note that the product theorem for cumulants (Theorem 3.67) and the fact that the means are zero imply

$$
\begin{aligned}
& \operatorname{cov}\left(I_{u_{j}, \omega_{1}}^{(T)}, I_{u_{j}, \omega_{2}}^{(T)}\right) \\
& =\frac{1}{4 \pi^{2} H_{2, N}(0)^{2}}\left[\operatorname{cum}\left(D_{u_{j}, \omega_{1}}^{(T)}, D_{u_{j},-\omega_{1}}^{(T)}, D_{u_{j},-\omega_{2}}^{(T)}, D_{u_{j}, \omega_{2}}^{(T)}\right)\right. \\
& \quad+S_{1423}\left(\operatorname{cum}\left(D_{u_{j}, \omega_{1}}^{(T)}, D_{u_{j}, \omega_{2}}^{(T)}\right) \otimes \operatorname{cum}\left(D_{u_{j},-\omega_{1}}^{(T)}, D_{u_{j},-\omega_{2}}^{(T)}\right)\right) \\
& \left.\quad+S_{1324}\left(\operatorname{cum}\left(D_{u_{j}, \omega_{1}}^{(T)}, D_{u_{j},-\omega_{2}}^{(T)}\right) \otimes \operatorname{cum}\left(D_{u_{j},-\omega_{1}}^{(T)}, D_{u_{j}, \omega_{2}}^{(T)}\right)\right)\right],
\end{aligned}
$$

where $S_{i j k l}$ denotes the permutation operator on $\otimes_{i=1}^{4} L_{\mathbb{C}}^{2}([0,1])$ that permutes the components of a tensor according to the permutation $(1,2,3,4) \mapsto(i, j, k, l)$, that is, $S_{i j k l}\left(x_{1} \otimes \cdots \otimes x_{4}\right)=x_{i} \otimes \cdots \otimes x_{l}$.

We first show that the first term of this expression is of lower order than the other two. By (3.40), the cumulant is equal to

$$
\begin{gathered}
\int_{\Pi^{4}} H_{N}\left(\mathcal{A}_{t_{j,}, \lambda_{1}}^{(T)}, \omega_{1}-\lambda_{1}\right) \otimes H_{N}\left(\mathcal{A}_{t_{j,}, \lambda_{2}}^{(T)},-\omega_{1}-\lambda_{2}\right) \otimes H_{N}\left(\mathcal{A}_{t_{j,}, \lambda_{3}}^{(T)},-\omega_{2}-\lambda_{3}\right) \\
\otimes H_{N}\left(\mathcal{A}_{t_{j,}, \lambda_{4}}^{(T)}, \omega_{2}-\lambda_{4}\right) \eta\left(\lambda_{1}+\ldots+\lambda_{4}\right) \mathcal{F}_{\lambda_{1}, \lambda_{2}, \lambda_{3}}^{\varepsilon} d \lambda_{1} \cdots d \lambda_{4}
\end{gathered}
$$


and hence, by Lemma A.3.3, is bounded in $L^{2}$-norm by

$$
\begin{gathered}
C \int_{\Pi^{3}} L_{N}\left(\omega_{1}-\lambda_{1}\right) L_{N}\left(-\omega_{1}-\lambda_{2}\right) L_{N}\left(-\omega_{2}-\lambda_{3}\right) L_{N}\left(\lambda_{1}+\lambda_{2}+\lambda_{3}+\omega_{2}\right) d \lambda 1 d \lambda_{2} d \lambda_{3} \\
\leqslant C \log (N)^{2} \int_{\Pi^{3}} L_{N}\left(\omega_{2}+\lambda_{3}\right)^{2} d \lambda_{3} \leqslant C N \log (N)^{2} .
\end{gathered}
$$

Next we consider the second term of (4.63). A similar derivation as for the expectation of the periodogram tensor shows that the term equals

$$
\begin{aligned}
& \int_{\Pi^{2}} H_{N}\left(\mathcal{A}_{t_{j, \boldsymbol{\bullet}}, \lambda_{1}}^{(T)}, \omega_{1}-\lambda_{1}\right) \otimes H_{N}\left(\mathcal{A}_{t_{j, \bullet},-\lambda_{1}}^{(T)}, \omega_{2}+\lambda_{1}\right) \otimes H_{N}\left(\mathcal{A}_{t_{j, \boldsymbol{\bullet}}, \lambda_{2}}^{(T)},-\omega_{1}-\lambda_{2}\right) \\
& \otimes H_{N}\left(\mathcal{A}_{t_{j, \bullet},-\lambda_{2}}^{(T)}, \lambda_{2}-\omega_{2}\right) \mathcal{F}_{\lambda_{1}}^{\varepsilon} \otimes \mathcal{F}_{\lambda_{2}}^{\varepsilon} d \lambda_{1} d \lambda_{2} \\
& =\int_{\Pi^{2}} H_{N}\left(\mathcal{A}_{u_{j, \bullet}, \lambda_{1}}, \omega_{1}-\lambda_{1}\right) \otimes H_{N}\left(\mathcal{A}_{u_{j, \bullet},-\lambda_{1}}, \omega_{2}+\lambda_{1}\right) \otimes H_{N}\left(\mathcal{A}_{u_{j, \bullet}, \lambda_{2}},-\omega_{1}-\lambda_{2}\right) \\
& \otimes H_{N}\left(\mathcal{A}_{u_{j, \bullet},-\lambda_{2}}, \lambda_{2}-\omega_{2}\right) \mathcal{F}_{\lambda_{1}}^{\varepsilon} \otimes \mathcal{F}_{\lambda_{2}}^{\varepsilon} d \lambda_{1} d \lambda_{2}+R_{T} \\
& =H_{2, N}\left(\mathcal{A}_{u_{j, \bullet}, \omega_{1}} \otimes \mathcal{A}_{u_{j, \bullet},-\omega_{1}}, \omega_{1}-\omega_{2}\right) \otimes H_{2, N}\left(\mathcal{A}_{u_{j, \bullet}, \omega_{1}} \otimes \mathcal{A}_{u_{j, \bullet},-\omega_{1}}, \omega_{2}-\omega_{1}\right) \\
& \times \mathcal{F}_{\omega_{1}}^{\varepsilon} \otimes \mathcal{F}_{-\omega_{1}}^{\varepsilon} \\
& =H_{2, N}\left(\mathcal{F}_{u_{j, \boldsymbol{\bullet}}, \omega_{1}}, \omega_{1}-\omega_{2}\right) \otimes H_{2, N}\left(\mathcal{F}_{u_{j, \boldsymbol{\bullet}},-\omega_{1}}, \omega_{2}-\omega_{1}\right)
\end{aligned}
$$

Proceeding in an analogous matter for the third term of (4.63), we obtain the stated result.

Proof of Theorem 3.5.6. Recall that by Theorem 3.5.4, the expectation of the periodogram tensor can be written as

$$
\mathbb{E}\left(I_{u_{j}, \omega}^{(T)}\right)=H_{2, N}\left(\mathcal{F}_{u_{j, \boldsymbol{\bullet}} \omega}, 0\right)+R_{T}=\frac{1}{H_{2, N}(0)} \sum_{r=1}^{N} h_{r, N}^{2} \mathcal{F}_{u_{j, r}, \omega}+O\left(\frac{\log (N)}{N}\right) .
$$

where the remainder term $R_{T}$ is of order $O\left(\frac{\log (N)}{N}\right)$. Because the operator-valued function $\mathcal{A}_{u, \omega}$ is twice differentiable with respect to both $u$ and $\omega$, it follows from Theorem A.1.8 that the tensor $\mathcal{F}_{u, \omega}$ is twice continuously differentiable in both $u$, and $\omega$. We can therefore apply a Taylor expansion of $\mathcal{F}_{u_{j, r}, \omega}$ about to the point $x=\left(u_{j}, \omega_{o}\right)$ to obtain

$$
\begin{aligned}
\mathcal{F}_{u_{j, r}, \omega} & =\mathcal{F}_{u_{j}, \omega_{o}}+\left.\left(\frac{r-N / 2}{T}\right) \frac{\partial}{\partial u} \mathcal{F}_{u, \omega}\right|_{(u, \omega)=x}+\left.(\omega-\lambda) \frac{\partial}{\partial \omega} \mathcal{F}_{u, \omega}\right|_{(u, \omega)=x} \\
& =\left.\frac{1}{2}\left(\frac{r-N / 2}{T}\right)^{2} \frac{\partial^{2}}{\partial u^{2}} \mathcal{F}_{u, \omega}\right|_{(u, \omega)=x}+\left.\frac{1}{2}(\omega-\lambda)^{2} \frac{\partial^{2}}{\partial \omega^{2}} \mathcal{F}_{u, \omega}\right|_{(u, \omega)=x} \\
& +\left(\frac{r-N / 2}{T}\right)(\omega-\lambda)\left(\left.\frac{\partial^{2}}{\partial u \partial \omega} \mathcal{F}_{u, \omega}\right|_{(u, \omega)=x}+\left.\frac{\partial^{2}}{\partial \omega \partial u} \mathcal{F}_{u, \omega}\right|_{(u, \omega)=x}\right)+R_{T, p},
\end{aligned}
$$

where the remainder can generally be bounded by

$$
\begin{aligned}
R_{T, p} & =\sum_{i_{1}, i_{2} \in \mathbb{N}: i_{1}+i_{2}>p} \frac{b_{\mathrm{t}, T}^{i_{1}}|\omega-\alpha|^{i_{2}}}{\left(i_{1}\right) !\left(i_{2}\right) !} \sup _{u, \omega}\left\|\frac{\partial^{i_{1}+i_{2}}}{\partial u^{i^{1}} \partial \omega^{i_{2}}} f_{u, \omega}\right\|_{2} \\
& =\sum_{i_{1}, i_{2} \in \mathbb{N}: i_{1}+i_{2}=p} O\left(b_{\mathrm{t}, T}^{i_{1}}|\omega-\alpha|^{i_{2}}\right) \quad p \geqslant 2 .
\end{aligned}
$$


In order to derive the mean of the estimator, we set $v b_{\mathrm{t}, T}=\frac{r-N / 2}{T}$ and recall that the taper function relates to a smoothing kernel $K_{\mathrm{t}}$ in time direction by

$$
K_{\mathrm{t}}(v)=\frac{1}{H_{2}} h^{2}\left(\frac{v+1}{2}\right)
$$

for $v \in\left[-\frac{1}{2}, \frac{1}{2}\right]$ with bandwidth $b_{\mathrm{t}, T}=N / T$. It then follows from (3.84) that a Taylor expansion about to the point $x=\left(u_{j}, \omega_{o}\right)$ yields

$$
\begin{aligned}
& \mathbb{E}\left(\hat{\mathcal{F}}_{u_{j}, \omega_{o}}^{(T)}\right)=\mathcal{F}_{u_{j}, \omega_{o}}+\left.\sum_{i=1}^{2} \frac{1}{i !} b_{\mathrm{t}, T}^{i} \int v^{i} K_{\mathrm{t}}(v) d v \int_{\Pi} K_{\mathrm{f}}(\alpha) d \alpha \frac{\partial^{i}}{\partial u^{i}} \mathcal{F}_{u, \omega}\right|_{(u, \omega)=x} \\
& +\left.\sum_{i=1}^{2} \frac{1}{i !} b_{\mathrm{f}, T}^{i} \int_{\Pi} \alpha^{i} K_{\mathrm{f}}(\alpha) d \alpha \int K_{\mathrm{t}}(v) d v \frac{\partial^{i}}{\partial \omega^{i}} \mathcal{F}_{u \omega}\right|_{(u, \omega)=x} \\
& +\frac{1}{2} b_{\mathrm{t}, T} b_{\mathrm{f}, T} \int v K_{\mathrm{t}}(v) d v \int_{\Pi} \alpha K_{\mathrm{f}}(\alpha) d \alpha\left(\left.\frac{\partial^{2}}{\partial u \partial \omega} \mathcal{F}_{u, \omega}\right|_{(u, \omega)=x}+\left.\frac{\partial^{2}}{\partial \omega \partial u} \mathcal{F}_{u, \omega}\right|_{(u, \omega)=x}\right)+R_{T, p} .
\end{aligned}
$$

Because the smoothing kernels are symmetric around 0, we obtain

$$
\begin{aligned}
\mathbb{E}\left(\hat{\mathcal{F}}_{u_{j}, \omega_{o}}^{(T)}\right) & =\mathcal{F}_{u_{j}, \omega_{o}}+\left.\frac{1}{2} b_{\mathrm{t}, T}^{2} \kappa_{\mathrm{t}} t \frac{\partial^{2}}{\partial u^{2}} \mathcal{F}_{u, \omega}\right|_{(u, \omega)=x}+\frac{1}{2} b_{\mathrm{f}, T}^{2} \kappa_{\mathrm{f}} 2,\left.1 \frac{\partial^{2}}{\partial \omega^{2}} \mathcal{F}_{u \omega}\right|_{(u, \omega)=x} \\
& +o\left(b_{\mathrm{t}, T}^{2}\right)+o\left(b_{\mathrm{f}, T}^{2}\right)+O\left(\frac{\log \left(b_{\mathrm{t}, T} T\right)}{b_{\mathrm{t}, T} T}\right)
\end{aligned}
$$

where the error terms follow from (3.85) and Theorem 3.5.4, respectively. This establishes Result $i$ ) of Theorem 3.5.6.

For the proof of the covariance structure, we note that

$$
\operatorname{cov}\left(\hat{\mathcal{F}}_{u, \omega_{1}}^{(T)}, \hat{\mathcal{F}}_{u, \omega_{2}}^{(T)}\right)=\int_{\Pi^{2}} K_{\mathrm{f}, T}\left(\omega_{1}-\lambda_{1}\right) K_{\mathrm{f}, T}\left(\omega_{2}-\lambda_{2}\right) \operatorname{cov}\left(I_{u, \lambda_{1}}^{(T)}, I_{u, \lambda_{2}}^{(T)}\right) d \lambda_{1} d \lambda_{2} .
$$

where by Theorem 3.5.4

$$
\begin{aligned}
\operatorname{cov}\left(I_{u, \lambda_{1}}^{(T)}, I_{u, \lambda_{2}}^{(T)}\right) \\
=\frac{1}{4 \pi^{2} H_{4, N}(0)^{2}}\left[S_{1423}\left(H_{2, N}\left(\mathcal{F}_{u_{\bullet}, \lambda_{1}}, \lambda_{1}-\lambda_{2}\right) \otimes H_{2, N}\left(\mathcal{F}_{u_{\bullet},-\lambda_{1}}, \lambda_{2}-\lambda_{1}\right)\right)\right. \\
\left.\quad+S_{1324}\left(H_{2, N}\left(\mathcal{F}_{u_{\bullet}, \lambda_{1}}, \lambda_{1}+\lambda_{2}\right) \otimes H_{2, N}\left(\mathcal{F}_{u_{\bullet},-\lambda_{1}},-\lambda_{1}-\lambda_{2}\right)\right)\right]+O\left(\frac{\log (N)}{N}\right) .
\end{aligned}
$$


We treat the two terms of the covariance tensor separately. Starting with the first term, we have

$$
\begin{gathered}
\| \int_{\Pi^{2}} K_{\mathrm{f}, T}\left(\omega_{1}-\lambda_{1}\right)\left[K_{\mathrm{f}, T}\left(\omega_{2}-\lambda_{2}\right)\left[H_{2, N}\left(F_{u_{\bullet}, \lambda_{1}}, \lambda_{1}-\lambda_{2}\right) \otimes H_{2, N}\left(\mathcal{F}_{u_{\bullet},-\lambda_{1}}, \lambda_{2}-\lambda_{1}\right)\right]\right. \\
\left.-K_{\mathrm{f}, T}\left(\omega_{2}-\lambda_{1}\right)\left|H_{2, N}\left(\lambda_{1}-\lambda_{2}\right)\right|^{2}\left(\mathcal{F}_{u, \lambda_{1}} \otimes \mathcal{F}_{u,-\lambda_{1}}\right)\right] d \lambda_{1} d \lambda_{2} \|_{2} \\
\leqslant \| \int_{\Pi^{2}} K_{\mathrm{f}, T}\left(\omega_{1}-\lambda_{1}\right) K_{\mathrm{f}, T}\left(\omega_{2}-\lambda_{2}\right)\left[H_{2, N}\left(F_{u_{\bullet}, \lambda_{1}}, \lambda_{1}-\lambda_{2}\right) \otimes H_{2, N}\left(\mathcal{F}_{u_{\bullet},-\lambda_{1}}, \lambda_{2}-\lambda_{1}\right)\right. \\
\left.-\left|H_{2, N}\left(\lambda_{1}-\lambda_{2}\right)\right|^{2}\left(\mathcal{F}_{u, \lambda_{1}} \otimes \mathcal{F}_{u,-\lambda_{1}}\right)\right] d \lambda_{1} d \lambda_{2} \|_{2} \\
+\| \int_{\Pi^{2}} K_{\mathrm{f}, T}\left(\omega_{1}-\lambda_{1}\right)\left[K_{\mathrm{f}, T}\left(\omega_{2}-\lambda_{2}\right)-K_{\mathrm{f}, T}\left(\omega_{2}-\lambda_{1}\right)\right] \\
\times\left|H_{2, N}\left(\lambda_{1}-\lambda_{2}\right)\right|^{2}\left(\mathcal{F}_{u, \lambda_{1}} \otimes \mathcal{F}_{u,-\lambda_{1}}\right) d \lambda_{1} d \lambda_{2} \|_{2}
\end{gathered}
$$

Since $\mathcal{F}_{u, \lambda}$ is uniformly Lipschitz continuous in $u$, we have $\left\|\mathcal{F}_{u_{r}, \lambda}-\mathcal{F}_{u, \lambda}\right\|_{2} \leqslant C \frac{N}{T}$ and hence the first term on the right-hand side is bounded by

$$
C \int_{\Pi^{2}} b_{\mathrm{f}, T}^{2} L_{\frac{1}{b_{\mathrm{f}, T}}}\left(\omega_{1}-\lambda_{1}\right)^{2} L_{\frac{1}{b_{\mathrm{f}, T}}}\left(\omega_{2}-\lambda_{2}\right)^{2} L_{N}\left(\lambda_{1}-\lambda_{2}\right)^{2} \frac{N}{T} d \lambda_{1} d \lambda_{2} \leqslant C \frac{N^{2}}{b_{\mathrm{f}, T} T}
$$

For the second term, we exploit uniform Lipschitz continuity of the kernel function $K_{\mathrm{f}}$ to get the upper bound

$$
C \int_{\Pi^{2}} K_{\mathrm{f}, T}\left(\omega_{1}-\lambda_{1}\right)^{2} b_{\mathrm{f}, T}^{-2}\left|\lambda_{1}-\lambda_{2}\right| L_{N}\left(\lambda_{1}-\lambda_{2}\right)^{2} d \lambda_{1} d \lambda_{2} \leqslant C \frac{\log (N)}{b_{\mathrm{f}, T}^{2}} .
$$

In total we obtain

$$
\left\|\operatorname{cov}\left(\hat{\mathcal{F}}_{u, \omega_{1}}^{(T)}, \hat{\mathcal{F}}_{u, \omega_{2}}^{(T)}\right)\right\|_{2}=O\left(\frac{\log (N)}{b_{\mathrm{f}, T}^{2} N^{2}}\right)+O\left(\frac{1}{b_{\mathrm{f}, T} T}\right)+O\left(\frac{\log (N)}{N}\right)
$$

uniformly in $\omega_{1}, \omega 2 \in[-\pi, \pi]$ and $u \in[0,1]$.

Proof of Proposition 3.5.13. We have

$$
\begin{aligned}
\operatorname{cum} & \left(\hat{E}_{u, \omega_{1}}^{(T)}\left(\psi_{m_{1} n_{1}}\right), \ldots, \hat{E}_{u, \omega_{k}}^{(T)}\left(\psi_{m_{k} n_{k}}\right)\right)=\frac{\left(b_{\mathrm{t}, T} b_{\mathrm{f}, T} T\right)^{k / 2}}{H_{2, N}(0)^{k}} \int_{\Pi^{k}} \prod_{j=1}^{k} K_{\mathrm{f}, T}\left(\omega_{j}-\lambda_{j}\right) \\
& \times \operatorname{cum}\left(D_{u, \omega_{1}}^{(T)}\left(\psi_{m_{1}}\right) D_{u,-\omega_{1}}^{(T)}\left(\psi_{n_{1}}\right), \ldots, D_{u, \omega_{k}}^{(T)}\left(\psi_{m_{k}}\right) D_{u,-\omega_{k}}^{(T)}\left(\psi_{n_{k}}\right)\right) d \lambda_{1} \cdots d \lambda_{k},
\end{aligned}
$$

where $D_{u, \omega}^{(T)}(\phi)=\left\langle D_{u, \omega}^{(T)}, \phi\right\rangle$ for $\phi \in L_{\mathbb{C}}^{2}([0,1])$. Application of the product theorem for cumulants (Theorem 3.67) yields for the cumulant

$$
\begin{array}{r}
\operatorname{cum}\left(D_{u, \omega_{1}}^{(T)}\left(\psi_{m_{1}}\right) D_{u,-\omega_{1}}^{(T)}\left(\psi_{n_{1}}\right), \ldots, D_{u, \omega_{k}}^{(T)}\left(\psi_{m_{k}}\right) D_{u,-\omega_{k}}^{(T)}\left(\psi_{n_{k}}\right)\right) \\
=\sum_{i . p .} \prod_{l=1}^{M} \operatorname{cum}\left(D_{u, \gamma_{p}}^{(T)}\left(\psi_{r_{p}}\right), p \in P_{l}\right),
\end{array}
$$


where the summation extends over all indecomposable partitions $P=\left\{P_{1}, \ldots, P_{M}\right\}$ of the table

$$
\begin{array}{cc}
(1,0) & (1,1) \\
\vdots & \vdots \\
(k, 0) & (k, 1)
\end{array}
$$

and, for $p=(i, j), \gamma_{p}=\gamma_{i j}=(-1)^{j} \lambda_{i}$ as well as $r_{p}=r_{i j}=m_{i}^{1-j} n_{i}^{j}$ for $i=1, \ldots, k$ and $j \in\{0,1\}$. For the next steps, we further denote the elements of $P_{l}$ with $\left|P_{l}\right|=d_{l}$ by $p_{l 1}, \ldots, p_{l d_{l}}$. Then, by (3.40), we obtain further for the above cumulant

$$
\begin{aligned}
\sum_{i . p .} \prod_{l=1}^{M} \int_{\Pi^{d_{l}-1}} \int_{[0,1]^{d_{l}}} & {\left[\bigotimes_{s=1}^{d_{l}} H_{N}\left(\mathcal{A}_{t_{u, \bullet}, \alpha_{s}}^{(T)}, \gamma_{p_{l s}}-\alpha_{s}\right) \mathcal{F}_{\alpha_{1}, \ldots, \alpha_{d_{l}-1}}^{\varepsilon}\right]\left(\tau_{1}, \ldots, \tau_{d_{l}}\right) } \\
& \times \prod_{s=1}^{d_{l}} \overline{\psi_{r_{p_{l s}}}\left(\tau_{s}\right)} d \tau_{1} \cdots d \tau_{d_{l}} \eta\left(\alpha_{1}+\ldots+\alpha_{d l}\right) d \alpha_{1} \cdots d \alpha_{d_{l}} .
\end{aligned}
$$

Noting that the inner integral is a inner product in the tensor product space, we get

$$
\begin{aligned}
& \left|\left\langle\bigotimes_{s=1}^{d_{l}} H_{N}\left(\mathcal{A}_{t_{u, \bullet}, \alpha_{s}}^{(T)}, \gamma_{p_{l s}}-\alpha_{s}\right) \mathcal{F}_{\alpha_{1}, \ldots, \alpha_{d_{l}-1}}^{\varepsilon}, \bigotimes_{s=1}^{d_{l}} \psi_{r_{p_{l s}}}\right\rangle\right| \\
& \leqslant\left\|\bigotimes_{s=1}^{d_{l}} H_{N}\left(\mathcal{A}_{t_{u, \bullet}, \alpha_{s}}^{(T)}, \gamma_{p_{l s}}-\alpha_{s}\right) \mathcal{F}_{\alpha_{1}, \ldots, \alpha_{d_{l}-1}}^{\varepsilon}\right\|_{2}\left\|\bigotimes_{s=1}^{d_{l}} \psi_{r_{p_{l s}}}\right\|_{2} \\
& \leqslant\left\|\bigotimes_{s=1}^{d_{l}} H_{N}\left(\mathcal{A}_{t_{u, \bullet}, \alpha_{s}}^{(T)}, \gamma_{p_{l s}}-\alpha_{s}\right)\right\|\left\|_{\infty}\right\| \mathcal{F}_{\alpha_{1}, \ldots, \alpha_{d_{l}-1}}^{\varepsilon}\left\|_{2} \prod_{s=1}^{d_{l}}\right\| \psi_{r_{p_{l s}}} \|_{2} .
\end{aligned}
$$

Noting that by Lemma A.3.3

$$
\left\|H_{N}\left(\mathcal{A}_{t_{u, \cdot}, \alpha_{s}}^{(T)}, \gamma_{p_{l s}}-\alpha_{s}\right)\right\|_{\infty} \leqslant K L_{N}\left(\gamma_{p_{l s}}-\alpha_{s}\right)
$$

for some constant $K$, we get together with $\left\|\mathcal{F}_{\alpha_{1}, \ldots, \alpha_{d_{l}-1}}^{\varepsilon}\right\|_{2} \leqslant K^{\prime}$ as an upper bound for $(3.90)$

$$
K \sum_{i . p .} \prod_{l=1}^{M} \int_{\Pi^{d_{l}}} \prod_{s=1}^{d_{l}} L_{N}\left(\gamma_{p_{l s}}-\alpha_{s}\right) \eta\left(\alpha_{1}+\ldots+\alpha_{d l}\right) d \alpha_{1} \cdots d \alpha_{d_{l}}
$$

and further by repeated use of Lemma A.3.1(v)

$$
\leqslant K \sum_{i . p .} \prod_{l=1}^{M} L_{N}\left(\bar{\gamma}_{l}\right) \log (N)^{d_{l}-1} \leqslant K \log (N)^{2 k-M} \sum_{i . p .} \prod_{l=1}^{M} L_{N}\left(\bar{\gamma}_{l}\right) .
$$

Substituting the upper bound for the cumulant in (3.88) and noting that $\frac{1}{N} H_{2, N}(0) \rightarrow$ $\|h\|_{2}^{2}$ as $N \rightarrow \infty$,we find

$$
\begin{aligned}
& \left|\operatorname{cum}\left(\hat{E}_{u, \omega_{1}}^{(T)}\left(\psi_{m_{1} n_{1}}\right), \ldots, \hat{E}_{u, \omega_{k}}^{(T)}\left(\psi_{m_{k} n_{k}}\right)\right)\right| \\
& \quad \leqslant \frac{C b_{\mathrm{f}, T}^{k / 2} \log (N)^{2 k-M}}{N^{k / 2}} \sum_{i . p .} \int_{\Pi^{k}} \prod_{j=1}^{k} K_{\mathrm{f}, T}\left(\omega_{j}-\lambda_{j}\right) \prod_{l=1}^{M} L_{N}\left(\bar{\gamma}_{l}\right) d \lambda_{1} \cdots d \lambda_{k} .
\end{aligned}
$$


It is sufficient to show that for each indecomposable partition $\left\{P_{1}, \ldots, P_{M}\right\}$ the corresponding term in the above sum tends to zero. First, suppose that $M=k$. Bounding the factors $K_{\mathrm{f}, T}\left(\omega_{i}-\lambda_{i}\right)$ by $\left\|K_{\mathrm{f}}\right\|_{\infty} / b_{\mathrm{f}, T}$ for $i=2, \ldots, k$ and integrating over $\lambda_{3}, \ldots, \lambda_{k}$, we obtain by Lemma A.3.2(i) as an upper bound

$$
\begin{aligned}
& \frac{C \log (N)^{2 k-M}}{b_{\mathrm{f}, T}^{k / 2-1} N^{k / 2}} \int_{\Pi^{2}} K_{\mathrm{f}, T}\left(\omega_{1}-\lambda_{1}\right) L_{N}\left(\lambda_{1} \pm \lambda_{2}\right)^{2} d \lambda_{1} d \lambda_{2} \\
& \leqslant \frac{C \log (N)^{2 k-2}}{b_{\mathrm{f}, T}^{k / 2-2} N^{k / 2}} \int_{\Pi^{2}} L_{b_{\mathrm{f}, T}^{-1}}\left(\omega_{1}-\lambda_{1}\right)^{2} L_{N}\left(\lambda_{1} \pm \lambda_{2}\right)^{2} d \lambda_{2} d \lambda_{1} \\
& \leqslant \frac{C \log (N)^{2 k-2}}{b_{\mathrm{f}, T}^{k / 2-2} N^{k / 2}} \int_{\Pi} N L_{b_{\mathrm{f}, T}^{-1}}\left(\omega_{1}-\lambda_{1}\right)^{2} d \lambda_{1} \leqslant \frac{C \log (N)^{2 k-2}}{\left(b_{\mathrm{f}, T} N\right)^{k / 2-1}},
\end{aligned}
$$

where we have $K_{\mathrm{f}, T}(\omega) \leqslant b_{\mathrm{f}, T} L_{b_{\mathrm{f}, T}^{-1}}(\omega)$ and repeatedly Lemma A.3.1(iv). Next, if $M<k$ we select variables $\lambda_{i_{1}}, \ldots, \lambda_{i_{k-2}}$ according to Lemma A.3.2(ii) and bound all corresponding factors $K_{\mathrm{f}, T}\left(\omega_{i_{j}}-\lambda_{i_{j}}\right)$ for $j=1, \ldots, k-2$ by $\left\|K_{\mathrm{f}}\right\|_{\infty} / b_{\mathrm{f}, T}$. Then integration over the $k-2$ selected variables yields the upper bound

$$
\begin{aligned}
& \frac{C \log (N)^{3 k-M-2}}{b_{\mathrm{f}, T}^{k / 2-2} N^{k / 2-1}} \int_{\Pi^{2}} K_{\mathrm{f}, T}\left(\omega_{i_{k-1}}-\lambda_{i_{k-1}}\right) K_{\mathrm{f}, T}\left(\omega_{i_{k}}-\lambda_{i_{k}}\right) d \lambda_{i_{k-1}} d \lambda_{i_{k}} \\
& \quad \leqslant \frac{C b_{\mathrm{f}, T} \log (N)^{3 k-M-2}}{b_{\mathrm{f}, T}^{k / 2-1} N^{k / 2-1}},
\end{aligned}
$$

since $\left\|K_{\mathrm{f}, T}\right\|_{1}=1$. Since $b_{\mathrm{f}, T} N=b_{\mathrm{f}, T} b_{\mathrm{t}, T} T \rightarrow \infty$ and $k / 2-1>0$, the upper bounds tend to zero as $T \rightarrow \infty$, which completes the proof. 



\section{Chapter 4}

\section{Testing for stationarity of functional time series in the frequency domain}

\subsection{Introduction}

The aim of the current chapter is to provide a new stationarity test for functional time series based on frequency domain methods. Particular attention is given to taking into account alternatives allowing for smooth variation as a source of nonstationarity, even though non-smooth alternatives can be covered as well. Functional data analysis has seen an upsurge in research contributions for at least one decade. This is reflected in the growing number of monographs in the area. Readers interested in the current state of statistical inference procedures may consult Bosq (2000), Ferraty and Vieu (2006), Horváth and Kokoszka (2012), Hsing and Eubank (2015) and Ramsay and Silverman (2005).

Papers on functional time series have come into the focus more recently and constitute now an active area of research. Hörmann and Kokoszka (2010) introduced a general weak dependence concept for stationary functional time series, while van Delft and Eichler (2016) provided a framework for locally stationary functional time series. Antoniadis and Sapatinas (2003), Aue et al. (2015) and Besse et al. (2000) constructed prediction methodology that may find application across many areas of science, economics and finance. With the exception of van Delft and Eichler (2016), the above contributions are concerned with procedures in the time domain. Other methodology in the frequency domain has been developed in parallel. One should mention Panaretos and Tavakoli (2013b), who provided results concerning the Fourier analysis of time series in function spaces, and Hörmann et al. (2015), who addressed the problem of dimension reduction for functional time series using dynamic principal components.

The methodology proposed in this chapter provides a new frequency domain inference procedure for functional time series. More precisely, a test for secondorder stationarity is developed. In the univariate case, such tests have a long history, going back at least to the seminal paper Priestley and Rao (1969), who based their 
method on the evaluation of evolutionary spectra of a given time series. Other contributions building on this work include von Sachs and Neumann (1999), who used local periodograms and wavelet analysis, and Paparoditis (2009), whose test is based on comparing a local estimate of the spectral density to a global estimate. In all three papers, interest is in smoothly varying alternatives. The same tests, however, also have power against non-smooth alternatives such as structural breaks or change-points. A recent review discussing methodology for structural breaks in time series is Aue et al. (2013).

The proposed test for second-order stationarity of functional time series uses the Discrete Fourier Transform (DFT). Its construction seeks to exploit that the DFTs of a functional time series evaluated at distinct Fourier frequencies are asymptotically uncorrelated if and only if the series is second-order stationary. The proposed method is therefore related to the work of Dwivedi and Subba Rao (2011), who put forth a similar test in a univariate framework. A different version of stationarity tests, based on time domain methodology involving cumulative sum statistics is given in Horváth and Rice (2014).

The proposed test is the first of its kind in the frequency domain analysis of functional time series. A delicate understanding of the functional DFT is needed in order to derive the asymptotic theory given here. In particular, results on the large sample behavior of a quadratic form test statistic are provided both under the null hypothesis of a stationary functional time series and the alternative of a locally stationary functional time series. For this, a weak convergence result is established that might be of interest in its own right, as it is verified using a simplified tightness criterion going back to work of Cremers and Kadelka (1986).

The remainder of the chapter is structured as follows. Section 4.2 provides the background, gives the requisite notations and introduces the functional version of the DFT. The exact form of the hypothesis test, model assumptions and the test statistic are introduced in Section 4.3. The large sample behavior under the null hypothesis of second-order stationarity and the alternative of local stationarity is established in Section 4.4. Empirical aspects are highlighted in Section 4.5. Finally, all technical details are relegated to the Appendix.

\subsection{Notation and setup}

\subsubsection{The function space}

A functional time series $\left(X_{t}: t \in \mathbb{Z}\right)$ will be viewed as a sequence of random elements on a probability space $(\Omega, \mathscr{A}, \mathbb{P})$ taking values in the separable Hilbert space of realvalued, square integrable functions on the unit interval $[0,1]$. This Hilbert space will be denoted by $H_{\mathbb{R}}=L^{2}([0,1], \mathbb{R})$. The functional DFT of $\left(X_{t}: t \in \mathbb{Z}\right)$, to be introduced in Section 4.2.3, can then be viewed as an element of $H_{\mathbb{C}}=L^{2}([0,1], \mathbb{C})$, the complex counterpart of $H_{\mathbb{R}}$. While the interval $[0,1]$ provides a convenient parametrization for the functions, the results of this chapter continue to hold for any separable Hilbert space. 
The complex conjugate of $z \in \mathbb{C}$ is denoted by $\bar{z}$ and the imaginary number by i. The inner product and the induced norm on $H_{\mathbb{C}}$ are given by

$$
\langle f, g\rangle=\int_{0}^{1} f(\tau) \overline{g(\tau)} d \tau \quad \text { and } \quad\|f\|_{2}=\sqrt{\langle f, f\rangle},
$$

respectively, for $f, g \in H_{\mathbb{C}}$. Two elements of $H_{\mathbb{C}}$ are tacitly understood to be equal if their difference has vanishing $L_{2}-$ norm. More generally, for functions $g:[0,1]^{k} \rightarrow \mathbb{C}$, the supremum norm is denoted by

$$
\|g\|_{\infty}=\sup _{\tau_{1}, \ldots, \tau_{k} \in[0,1]}\left|g\left(\tau_{1}, \ldots, \tau_{k}\right)\right|
$$

and the $L^{p}$-norm by

$$
\|g\|_{p}=\left(\int_{[0,1]^{k}}\left|g\left(\tau_{1}, \ldots, \tau_{k}\right)\right|^{p} d \tau_{1} \cdots d \tau_{k}\right)^{1 / p} .
$$

In all of the above, the obvious modifications apply to $H_{\mathbb{R}}$, the canonical Hilbert space in the functional data analysis setting.

Let $H$ stand for either $H_{\mathbb{R}}$ or $H_{\mathbb{C}}$. An operator $A$ on $H$ is said to be compact if its pre-image is compact. A compact operator admits a singular value decomposition

$$
A=\sum_{n=1}^{\infty} s_{n}(A) \psi_{n} \otimes \phi_{n}
$$

where $\left(s_{n}(A): n \in \mathbb{N}\right)$, are the singular values of $A,\left(\phi_{n}: n \in \mathbb{N}\right)$ and $\left(\psi_{n}: n \in \mathbb{N}\right)$ orthonormal bases of $H$ and $\otimes$ denoting the tensor product. The singular values are ordered to form a monotonically decreasing sequence of non-negative numbers. Based on the convergence rate to zero, operators on $H$ can be classified into particular Schatten $p$-classes. That is, for $p \geqslant 1$, the Schatten $p$-class $S_{p}(H)$ is the subspace of all compact operators $A$ on $H$ such that the sequence $s(A)=\left(s_{n}(A): n \in \mathbb{N}\right)$ of singular values of $A$ belongs to the sequence space $\ell^{p}$, that is,

$$
A \in S_{p}(H) \quad \text { if and only if } \quad\|A\|_{p}=\left(\sum_{n=1}^{\infty} s_{n}^{p}(A)\right)^{1 / p}<\infty,
$$

where $\|A\|_{p}$ is referred to as the Schatten $p-n o r m$. The space $S_{p}(H)$ together with the norm $\|A\|_{p}$ forms a Banach space and a Hilbert space in case $p=2$. By convention, the space $S_{\infty}(H)$ indicates the space of bounded linear operators equipped with the standard operator norm. For $1 \leqslant p \leqslant q$, the inclusion $S_{p}(H) \subseteq S_{q}(H)$ is valid. Two important classes are the nuclear or Trace-class operators and the Hilbert-Schmidt operators on $H$, which are given by $S_{1}(H)$ and $S_{2}(H)$, respectively. More properties of Schatten class operators, in particular of Hilbert-Schmidt operators, are provided in van Delft and Eichler (2016). The adjoint of $A$ is denoted by $A^{\dagger}$. Finally, we mention that the identity and zero operator on $H$ are denoted by $I_{H}$ and $O_{H}$, respectively. 


\subsubsection{Dependence structure on the function space}

A functional time series $X=\left(X_{t}: t \in \mathbb{Z}\right)$ is called strictly stationary if, for all finite sets of indices $J \subset \mathbb{Z}$, the joint distribution of $\left(X_{t+j}: j \in J\right)$ does not depend on $t \in \mathbb{Z}$. Similarly, $X$ is weakly stationary if its first- and second-order moments exist and are invariant under translation in time. In the case that they exist, define the mean function $m$ of $X$ point-wise by setting $m(\tau)=\mathbb{E}\left[X_{t}(\tau)\right], \tau \in[0,1]$, and the autocovariance kernel $c_{h}$ at lag $h \in \mathbb{Z}$ by

$$
c_{h}\left(\tau, \tau^{\prime}\right)=\operatorname{cov}\left(X_{t+h}(\tau), X_{t}\left(\tau^{\prime}\right)\right), \quad \tau, \tau^{\prime} \in[0,1] .
$$

Both $m$ and $c_{h}$ are well defined in the $L^{2}$ sense if $\mathbb{E}\left[\left\|X_{0}\right\|_{2}^{2}\right]<\infty$. Each kernel $c_{h}$ induces a corresponding autocovariance operator $\mathcal{C}_{h}$ on $H_{\mathbb{R}}$ by

$$
\mathcal{C}_{h} g(\tau)=\int_{0}^{1} c_{h}\left(\tau, \tau^{\prime}\right) g\left(\tau^{\prime}\right) d \tau^{\prime}=\mathbb{E}\left[\left\langle g, X_{0}\right\rangle X_{h}(\tau)\right],
$$

for all $g \in H_{\mathbb{R}}$. In analogy to weakly stationary multivariate time series, where the covariance matrix and spectral density matrix form a Fourier pair, the spectral density operator $\mathcal{F}_{\omega}$ is given by the Fourier transform of $\mathcal{C}_{h}$,

$$
\mathcal{F}_{\omega}=\frac{1}{2 \pi} \sum_{h \in \mathbb{Z}} \mathcal{C}_{h} e^{-\mathrm{i} \omega h}
$$

A sufficient condition for the existence of $\mathcal{F}_{\omega}$ in $S_{p}\left(H_{\mathbb{C}}\right)$ is $\sum_{h \in \mathbb{Z}}\left\|\mathcal{C}_{h}\right\|_{p}<\infty$.

In the current chapter, no structural modeling conditions, for example along the lines of the weak dependence notion in Hörmann and Kokoszka (2010) are imposed. Rather, as in Panaretos and Tavakoli (2013b), higher order dependence among the functional observations is defined through cumulant mixing conditions (Brillinger, 1981; Brillinger and Rosenblatt, 1967). For this, the notion of higher order cumulant tensors is required; see Appendix B.1 for a discussion on their properties. Point-wise, the $k$-th order cumulant function of the process $X$ can be defined by

$$
c_{t_{1}, \ldots, t_{k-1}}\left(\tau_{1}, \ldots, \tau_{k}\right)=\sum_{\nu=\left(\nu_{1}, \ldots, \nu_{p}\right)}(-1)^{p-1}(p-1) ! \prod_{l=1}^{p} \mathbb{E}\left[\prod_{j \in \nu_{l}} X_{t_{j}}\left(\tau_{j}\right)\right],
$$

where the summation is over all unordered partitions of $\{1, \ldots, k\}$. The quantity in (4.6) will be referred to as the $k$-th order cumulant kernel if it is properly defined in the $L^{2}$ sense. A sufficient condition for this to be satisfied is $\mathbb{E}\left[\left\|X_{0}\right\|_{2}^{k}\right]<\infty$. The cumulant kernel $c_{t_{1}, \ldots, t_{2 k-1}}\left(\tau_{1}, \ldots, \tau_{2 k}\right)$ induces a $2 k$-th order cumulant operator $\mathcal{C}_{t_{1}, \ldots, t_{2 k-1}}$ through right integration:

$$
\mathcal{C}_{t_{1}, \ldots, t_{2 k-1}} g\left(\tau_{1}, \ldots, \tau_{k}\right)=\int_{[0,1]^{k}} c_{t_{1}, \ldots, t_{2 k-1}}\left(\tau_{1}, \ldots, \tau_{2 k}\right) g\left(\tau_{k+1}, \ldots, \tau_{2 k}\right) d \tau_{k+1} \cdots d \tau_{2 k},
$$

which maps from $L^{2}\left([0,1]^{k}, \mathbb{R}\right)$ to $L^{2}\left([0,1]^{k}, \mathbb{R}\right)$. Similar to the case $k=2$, this operator will form a Fourier pair with a $2 k$-th order cumulant spectral operator 
given summability with respect to $\|\cdot\|_{p}$ is satisfied. The $2 k$-th order cumulant spectral operator is specified as

$$
\mathcal{F}_{\omega_{1}, \ldots, \omega_{2 k-1}}=(2 \pi)^{1-2 k} \sum_{t_{1}, \ldots, t_{2 k-1} \in \mathbb{Z}} \mathcal{C}_{t_{1}, \ldots, t_{2 k-1}} \exp \left(-\mathrm{i} \sum_{j=1}^{2 k-1} \omega_{j} t_{j}\right)
$$

where the convergence is in $\|\cdot\|_{p}$. Under suitable regularity conditions, the corresponding kernels also form a Fourier pair.

\subsubsection{The functional Discrete Fourier Transform}

Let $\left(X_{t}: t \in \mathbb{Z}\right)$ be a zero-mean, $H_{\mathbb{R}}$-valued stochastic process of which the stretch $X_{1}, \ldots, X_{T}$ is observed. In a similar way as one defines the DFT for univariate time series, the functional Discrete Fourier Transform (fDFT) operator $D_{\omega}^{(T)}$ at frequency $\omega$ is set up point-wise by letting

$$
D_{\omega}^{(T)}(\tau)=\frac{1}{\sqrt{2 \pi T}} \sum_{t=1}^{T} X_{t}(\tau) e^{-\mathrm{i} \omega t}, \quad \tau \in[0,1]
$$

If $\mathbb{E}\left[\|X\|_{2}^{2}\right]<\infty$, the periodogram operator at frequency $\omega$ is then defined as the tensor product of $D_{\omega}^{(T)}$ in $(4.8)$ on $H_{\mathbb{C}}$ with its adjoint $\left[D_{\omega}^{(T)}\right]^{\dagger}$, that is,

$$
I_{\omega}^{(T)}=\left[D_{\omega}^{(T)}\right] \otimes\left[D_{\omega}^{(T)}\right]^{\dagger} .
$$

Panaretos and Tavakoli (2013b) gave a generalization of Theorem 4.4.1 in Brillinger (1981) on periodogram matrices to the operator setting. Their Theorem 2.2 is the starting point for the considerations of this chapter. It states that, under weak dependence conditions expressed through higher order cumulants, the fDFTs evaluated at distinct frequencies yield asymptotically independent Gaussian random elements in $H_{\mathbb{C}}$. It shows therefore that the fDFT sequence of a Hilbert space stationary process is asymptotically uncorrelated at the canonical frequencies $\omega_{j}=2 \pi j / T$. This fundamental result will be extended to locally stationary functional time series in the current chapter. In particular, a representation of the cumulant kernel of the fDFT sequence in terms of the (time-varying) spectral density kernel is given. For weakly stationary processes, it will turn out in particular that, for $j \neq j^{\prime}$ or $j \neq T-j^{\prime}$, the covariance of the fDFT satisfies $\operatorname{cov}\left(D_{\omega_{j}}^{(T)}, D_{\omega_{j^{\prime}}}^{(T)}\right)=O(1 / T)$. The reverse argument (uncorrelatedness of the functional DFT sequence implies weak stationarity) can be shown by means of the inverse fDFT

$$
X_{t}(\tau)=\sqrt{\frac{2 \pi}{T}} \sum_{j=0}^{T-1} D_{\omega_{j}}^{(T)}(\tau) e^{\mathrm{i} \omega_{j} t}, \quad \tau \in[0,1] .
$$


Using this expression, the point-wise covariance kernel of $X_{t}$ and $X_{t^{\prime}}$ in terms of the fDFT sequence can be written as

$$
\begin{aligned}
c_{t, t^{\prime}}\left(\tau, \tau^{\prime}\right) & =\mathbb{E}\left[X_{t}(\tau) X_{t^{\prime}}\left(\tau^{\prime}\right)\right] \\
& =\frac{2 \pi}{T} \sum_{j, j^{\prime}=0}^{T-1} \mathbb{E}\left[D_{\omega_{j}}^{(T)}(\tau), \overline{D_{\omega_{j^{\prime}}}^{(T)}\left(\tau^{\prime}\right)}\right] e^{\mathrm{i} t \omega_{j}-\mathrm{i} t^{\prime} \omega_{j^{\prime}}} \\
& =\frac{2 \pi}{T} \sum_{j=0}^{T-1} \mathbb{E}\left[I_{\omega_{j}}^{(T)}\left(\tau, \tau^{\prime}\right)\right] e^{\mathrm{i} \omega_{j}\left(t-t^{\prime}\right)} \\
& =c_{t-t^{\prime}}\left(\tau, \tau^{\prime}\right),
\end{aligned}
$$

where the equality in (4.10) holds when $\mathbb{E}\left[D_{\omega_{j}}^{(T)}(\tau) \overline{D_{\omega_{j^{\prime}}}^{(T)}\left(\tau^{\prime}\right)}\right]=0$ with $j \neq j^{\prime}$ or $j \neq T-j^{\prime}$. This demonstrates that the autocovariance kernel of a second-order stationary functional time series is obtained and, hence, that an uncorrelated DFT sequence implies second-order stationarity up to lag $T$. Below, the behavior of the fDFT under the smooth alternative of locally stationary functional time series is derived. Our findings thus extend the results of Dwivedi and Subba Rao (2011) to the functional time series setting.

\subsection{The functional stationarity testing framework}

This section gives precise formulations of the hypotheses of interest, states the main assumptions used throughout the chapter and introduces the test statistic. Throughout, interest is in testing the null hypothesis

$$
H_{0}:\left(X_{t}: t \in \mathbb{Z}\right) \text { is a stationary functional time series }
$$

versus the alternative

$$
H_{A}:\left(X_{t}: t \in \mathbb{Z}\right) \text { is a locally stationary functional time series. }
$$

The definition of locally stationary functional time series is taken from van Delft and Eichler (2016).

Definition 4.3.1. A stochastic process $\left(X_{t}: t \in \mathbb{Z}\right)$ taking values in $H_{\mathbb{R}}$ is said to be locally stationary if

(1) $X_{t}=X_{t}^{(T)}$ for $t=1, \ldots, T$ and $T \in \mathbb{N}$; and

(2) for any rescaled time $u=\frac{t}{T} \in[0,1]$, there is a strictly stationary process $\left(X_{t}^{(u)}: t \in \mathbb{Z}\right)$ such that

$$
\left\|X_{t}^{(T)}-X_{t}^{(u)}\right\|_{2} \leqslant\left(\left|\frac{t}{T}-u\right|+\frac{1}{T}\right) P_{t, T}^{(u)} \quad \text { a.s. }
$$

where $P_{t, T}^{(u)}$ is a positive, real-valued triangular array of random variables such that, for some $\rho>0$ and $C<\infty, \mathbb{E}\left[\left|P_{t, T}^{(u)}\right|^{\rho}\right]<\infty$ for all $t$ and $T$, uniformly in $u \in[0,1]$. 
Note that, under $H_{A}$, the process constitutes a triangular array of functions. Inference methods are then based on infill asymptotics as popularized in Dahlhaus (1997) for univariate time series. The process is therefore observed on a finer grid as $T$ increases and more observations are available at a local level. A rigorous statistical framework for locally stationary functional time series was recently provided in van Delft and Eichler (2016). These authors established in particular that linear functional time series can be defined by means of a time-varying functional Cramér representation and provided sufficient conditions in the frequency domain for the above definition to be satisfied.

Based on the observations in Section 4.2.3, a test for weak stationarity can be set up exploiting the uncorrelatedness of the elements in the sequence $\left(D_{\omega_{j}}^{(T)}\right)$. Standardizing these quantities is a non-trivial task as the spectral density operators $\mathcal{F}_{\omega_{j}}^{(T)}$ are not only unknown but generally not globally invertible. Here, a statistic based on projections is considered. Let $\left(\psi_{l}: l \in \mathbb{N}\right)$ be an orthonormal basis of $H_{\mathbb{C}}$. Then, $\left(\psi_{l} \otimes \psi_{l^{\prime}}: l, l^{\prime} \in \mathbb{N}\right)$ is an orthonormal basis of $L^{2}\left([0,1]^{2}, \mathbb{C}\right)$ and, by definition of the Hilbert Schmidt inner product on the algebraic tensor product space $H \otimes_{\text {alg }} H$,

$$
\begin{aligned}
\left\langle\mathbb{E}\left(D_{\omega_{j_{1}}}^{(T)} \otimes D_{\omega_{j_{2}}}^{(T)}\right), \psi_{l} \otimes \psi_{l^{\prime}}\right\rangle_{H \otimes H} & =\mathbb{E}\left[\left\langle D_{\omega_{j_{1}}}^{(T)}, \psi_{l}\right\rangle\left\langle D_{\omega_{j_{2}}}^{(T)}, \psi_{l^{\prime}}\right\rangle\right] \\
& =\operatorname{cov}\left(\left\langle D_{\omega_{j_{1}}}^{(T)}, \psi_{l}\right\rangle, \overline{\left\langle D_{\omega_{j_{2}}}^{(T)}, \psi_{l^{\prime}}\right\rangle}\right) .
\end{aligned}
$$

This motivates to set up a test statistics based on the quantities

$$
\gamma_{h}^{(T)}\left(l, l^{\prime}\right)=\frac{1}{T} \sum_{j=1}^{T} \frac{\left\langle D_{\omega_{j}}^{(T)}, \psi_{l}\right\rangle \overline{\left\langle D_{\omega_{j+h}}^{(T)}, \psi_{l^{\prime}}\right\rangle}}{\sqrt{\left\langle\mathcal{F}_{\omega_{j}}\left(\psi_{l}\right), \psi_{l}\right\rangle\left\langle\mathcal{F}_{\omega_{j+h}}^{\dagger}\left(\psi_{l^{\prime}}\right), \psi_{l^{\prime}}\right\rangle}}, \quad h=1, \ldots, T-1 .
$$

In practice, the unknown spectral density operators $\mathcal{F}_{\omega_{j}}$ and $\mathcal{F}_{\omega_{j+h}}$ are to be replaced with consistent estimators $\hat{\mathcal{F}}_{\omega_{j}}^{(T)}$ and $\hat{\mathcal{F}}_{\omega_{j+h}}^{(T)}$. The corresponding estimated quantity will be denoted by $\hat{\gamma}_{h}^{(T)}$. As an estimator of $\mathcal{F}_{\omega}^{(T)}$, take

$$
\hat{\mathcal{F}}_{\omega}^{(T)}=\frac{2 \pi}{T} \sum_{j=1}^{T} K_{b}\left(\omega-\omega_{j}\right)\left(\left[D_{\omega_{j}}^{T}\right] \otimes\left[D_{\omega_{j}}^{T}\right]^{\dagger}\right),
$$

where $K_{b}(\cdot)=\frac{1}{b} K(\dot{\bar{b}})$ is a window function satisfying the following conditions.

Assumption 4.3.2. Let $K:\left[-\frac{1}{2}, \frac{1}{2}\right] \rightarrow \mathbb{R}$ be a positive, symmetric window function with $\int K(x) d x=1$ and $\int K(x)^{2} d x<\infty$ that is periodically extended, i.e., $K_{b}(x)=$ $\frac{1}{b} K\left(\frac{x \pm 2 \pi}{b}\right)$.

The periodic extension is to include estimates for frequencies around $\pm \pi$. Further conditions on the bandwidth $b$ are imposed below to determine the large sample behavior of $\hat{\gamma}_{h}^{(T)}$ under both the null and the alternative; see Section 4.4 below for details. The replacement of the unknown operators with consistent estimators requires to derive the order of the difference

$$
\sqrt{T}\left|\gamma_{h}^{(T)}-\hat{\gamma}_{h}^{(T)}\right|
$$


It will be shown in the next section that, for appropriate choices of the bandwidth $b$, this term is negligible under both null and alternative hypothesis.

To set up the test statistic, it now appears reasonable to extract information across a range of directions $l, l^{\prime}=1, \ldots, L$ and a selection of lags $h=1, \ldots, \bar{h}$, where $\bar{h}$ denotes an upper limit. Build therefore first the $L \times L \operatorname{matrix} \hat{\Gamma}_{h}^{(T)}=$ $\left(\hat{\gamma}_{h}^{(T)}\left(l, l^{\prime}\right): l, l^{\prime}=1, \ldots, L\right)$ and construct the vector

$$
\hat{\gamma}_{h}^{(T)}=\operatorname{vec}\left(\hat{\Gamma}_{h}^{(T)}\right)
$$

by vectorizing $\hat{\Gamma}_{h}^{(T)}$ via stacking of its columns. Define a scaled version of $\hat{\gamma}_{h}^{(T)}$ by

$$
\hat{\boldsymbol{\beta}}_{h}^{(T)}=\boldsymbol{e}^{\top} \hat{\gamma}_{h}^{(T)}
$$

where $\boldsymbol{e}$ is a vector of dimension $L^{2}$ whose elements are all equal to one and ${ }^{\top}$ denotes transposition. Choose now a collection $h_{1}, \ldots, h_{M}$ of lags each of which is upper bounded by $\bar{h}$ to pool information across a number of autocovariances and build the vectors

$$
\sqrt{T} \hat{\boldsymbol{b}}_{M}^{(T)}=\sqrt{T}\left(\Re \hat{\boldsymbol{\beta}}_{h_{1}}^{(T)}, \ldots, \Im \hat{\boldsymbol{\beta}}_{h_{M}}^{(T)}, \Im \hat{\boldsymbol{\beta}}_{h_{1}}^{(T)}, \ldots, \Im \hat{\boldsymbol{\beta}}_{h_{M}}^{(T)}\right)^{\top},
$$

where $\Re$ and $\Im$ denote real and imaginary part, respectively. Finally, set up the quadratic form

$$
\hat{Q}_{M}^{(T)}=\left(\hat{\boldsymbol{b}}_{M}^{(T)}\right)^{\top} \hat{\Sigma}_{M}^{-1} \hat{\boldsymbol{b}}_{M}^{(T)},
$$

where $\hat{\Sigma}_{M}$ is an estimator of the asymptotic covariance matrix of the vectors $\boldsymbol{b}_{M}^{(T)}$ which are defined by replacing $\hat{\gamma}_{h}^{(T)}$ with $\gamma_{h}^{(T)}$ in the definition of $\hat{\boldsymbol{b}}_{M}^{(T)}$. The statistic $\hat{Q}_{M}^{(T)}$ will be used to test the null of stationarity against the alternative of local stationarity. Note that this quadratic form depends on the tuning parameters $L$ and $M$.

\subsection{Large sample results}

\subsubsection{Properties under the null of stationarity}

The following gives the main requirements under stationarity of the functional time series that are needed to establish the asymptotic behavior of the test statistic under the null hypothesis.

Assumption 4.4.1 (Stationary functional time series). Let $\left(X_{t}: t \in \mathbb{Z}\right)$ be a stationary functional time series with values in $H_{\mathbb{R}}$ such that

(i) $\mathbb{E}\left[\left\|X_{0}\right\|_{2}^{k}\right]<\infty$,

(ii) $\sum_{t_{1}, \ldots, t_{k-1}=-\infty}^{\infty}\left(1+\left|t_{j}\right|^{\ell}\right)\left\|c_{t_{1}, \ldots, t_{k-1}}\right\|_{2}<\infty$ for all $1 \leqslant j \leqslant k-1$,

for some fixed values of $k, \ell \in \mathbb{N}$. 
The conditions of Assumption 4.4.1 ensure that the $k$-th order cumulant spectral density kernel

$$
f_{\omega_{1}, \ldots, \omega_{k-1}}\left(\tau_{1}, \ldots, \tau_{k}\right)=\frac{1}{(2 \pi)^{k-1}} \sum_{t_{1}, \ldots, t_{k-1}=-\infty}^{\infty} c_{t_{1}, \ldots, t_{k-1}}\left(\tau_{1}, \ldots, \tau_{k}\right) e^{-\mathrm{i}\left(\sum_{j=1}^{k} \omega_{j} t_{j}\right)}
$$

is well-defined in $L^{2}$ and is uniformly continuous in $\omega$ with respect to $\|\cdot\|_{2}$. Additionally, the parameter $\ell$ controls the smoothness of $f_{\omega}$ in the sense that, for all $i \leqslant \ell$,

$$
\sup _{\omega}\left\|\frac{\partial^{i}}{\partial \omega^{i}} f_{\omega}\right\|_{2}<\infty .
$$

A proof of these facts can be found in Panaretos and Tavakoli (2013b); van Delft and Eichler (2016). As in Section 4.2.2, through right-integration (4.16) induces a $k$-th order cumulant spectral density operator $\mathcal{F}_{\omega_{1}, \ldots, \omega_{k-1}}$ which is Hilbert-Schmidt. The following theorem establishes that the scaled difference between $\gamma_{h}^{(T)}$ and $\hat{\gamma}_{h}^{(T)}$ is negligible in large samples.

Theorem 4.4.2. Let Assumptions 4.3.2 and 4.4.1 be satisfied with $k=8$ and $\ell=2$ and assume further that $\inf _{\omega}\langle\mathcal{F}(\psi), \psi\rangle>0$ for all $\psi \in H$. Then, for any fixed $h$,

$$
\sqrt{T}\left|\gamma_{h}^{(T)}-\hat{\gamma}_{h}^{(T)}\right|=O_{p}\left(\frac{1}{\sqrt{b T}}+b^{2}\right) \quad(T \rightarrow \infty) .
$$

The proof is given in Appendix B.2. Theorem 4.4.2 shows that the distributional properties of $\gamma_{r}^{(T)}$ are asymptotically the same as those of $\hat{\gamma}_{h}^{(T)}$, provided that the following extra condition on the bandwidth hold.

Assumption 4.4.3. The bandwidth $b$ satisfies $b \rightarrow 0$ such that $b T \rightarrow \infty$ as $T \rightarrow \infty$.

We note that these are in fact the rates necessary for the estimator (4.12) to be consistent (Panaretos and Tavakoli, 2013b) and therefore do not impose an additional constraint under $H_{0}$. The next theorem derives the second-order structure of $\gamma_{h}^{(T)}$. It shows that the asymptotic variance is uncorrelated for all lags $h$ and that there is no correlation between the real and imaginary parts. For any $z \in \mathbb{C}$ denote by $\Re z$ and $\Im z$ its real and imaginary part, respectively.

Theorem 4.4.4. Let Assumption 4.4.1 be satisfied with $k=\{2,4\}$. Then,

$$
\begin{aligned}
& T \operatorname{cov}\left(\Re \gamma_{h_{1}}^{(T)}\left(l_{1}, l_{1}^{\prime}\right), \Re \gamma_{h_{2}}^{(T)}\left(l_{2}, l_{2}^{\prime}\right)\right) \\
& =T \operatorname{cov}\left(\Im \gamma_{h_{1}}^{(T)}\left(l_{1}, l_{1}^{\prime}\right), \Im \gamma_{h_{2}}^{(T)}\left(l_{2}, l_{2}^{\prime}\right)\right) \\
& =\left\{\begin{array}{cc}
\frac{1}{4 \pi}\left(\int \frac{\mathcal{F}_{\omega}^{\left(l_{1}, l_{2}\right)} \mathcal{F}_{-\omega-\omega_{h}}^{\left(l_{1}^{\prime}, l_{2}^{\prime}\right)}}{\sqrt{\mathcal{F}_{\omega}^{\left(l_{1}, l_{1}\right)} \mathcal{F}_{-\omega}^{\left(l_{2}, l_{2}\right)} \mathcal{F}_{-\omega-\omega_{h}}^{\left(l_{\prime}^{\prime}, l_{\prime}^{\prime}\right)} \mathcal{F}_{\omega+\omega_{h}}^{\left(l_{2}^{\prime}, l_{2}^{\prime}\right)}}} d \omega+\int \frac{\mathcal{F}_{\omega}^{\left(l_{1}, l_{2}^{\prime}\right)} \mathcal{F}_{-\omega-\omega_{h}}^{\left(l_{1}^{\prime}, l_{2}\right)}}{\sqrt{\mathcal{F}_{\omega}^{\left(l_{1}, l_{1}\right)} \mathcal{F}_{-\omega}^{\left(l_{2}^{\prime}, l_{2}^{\prime}\right)} \mathcal{F}_{-\omega-\omega_{h}}^{\left(l_{1}^{\prime}, l_{1}^{\prime}\right)} \mathcal{F}_{\omega+\omega_{h}}^{\left(l_{2}, l_{2}\right)}}} d \omega\right. \\
\left.\quad+\iint \frac{\mathcal{F}_{\omega,-\omega-\omega_{h},-\omega^{\prime}}^{\left(l_{1} l_{1}^{\prime}, l_{2} l_{2}^{\prime}\right)}}{\sqrt{\mathcal{F}_{\omega}^{\left(l_{1}, l_{1}\right)} \mathcal{F}_{-\omega-\omega_{h}}^{\left(l_{1}^{\prime}, l_{1}^{\prime}\right)} \mathcal{F}_{-\omega^{\prime}}^{\left(l_{2}, l_{2}\right)} \mathcal{F}_{\omega^{\prime}+\omega_{h}}^{\left(l_{2}^{\prime}, l_{2}^{\prime}\right)}}} d \omega d \omega^{\prime}\right), & \text { if } h_{1}=h_{2}=h . \\
O\left(\frac{1}{T}\right), & \text { if } h_{1} \neq h_{2} .
\end{array}\right.
\end{aligned}
$$


where $\mathcal{F}_{\omega}^{\left(l, l^{\prime}\right)}=\left\langle\mathcal{F}_{\omega}\left(\psi_{l^{\prime}}\right), \psi_{l}\right\rangle, \mathcal{F}_{\omega_{1}, \omega_{2}, \omega_{3}}^{\left(l_{1} l_{1}^{\prime}, l_{2} l_{2}^{\prime}\right)}=\left\langle\mathcal{F}_{\omega_{1}, \omega_{2}, \omega_{3}}\left(\psi_{l_{2} l_{2}^{\prime}}\right), \psi_{l_{1} l_{1}^{\prime}}\right\rangle$ and $\psi_{l l^{\prime}}=\psi_{l} \otimes \psi_{l^{\prime}}$. Furthermore,

$$
T \operatorname{cov}\left(\Re \gamma_{h_{1}}^{(T)}\left(l_{1}, l_{1}^{\prime}\right), \Im \gamma_{h_{2}}^{(T)}\left(l_{2}, l_{2}^{\prime}\right)\right)=O\left(T^{-1}\right)
$$

uniformly in $h_{1}, h_{2} \in \mathbb{Z}$.

The proof of Theorem 4.4.4 is given in Appendix B.5. Note that the results in the theorem use at various places the fact that the $k$-th order spectral density operator evaluated at $\boldsymbol{\lambda}=\left(\lambda_{1}, \ldots, \lambda_{k}\right)^{\top} \in \mathbb{R}^{k}$ equals the $k$-th order spectral density operator evaluated at $-\boldsymbol{\lambda}$ in the manifold $\sum_{j=1}^{k} \lambda_{j} \equiv 0 \bmod 2 \pi$.

With the previous results in place, the large sample behavior of the quadratic form statistics $\hat{Q}_{M}^{(T)}$ defined in (4.15) can be derived. This is done in the following theorem.

Theorem 4.4.5. Let Assumptions 4.3.2 and 4.4.3 be satisfied. Let Assumption 4.4.1 be satisfied with $k=8$ and $\ell=2$ and assume that $\inf _{\omega}\left\langle\mathcal{F}_{\omega}(\psi), \psi\right\rangle>0$ for all $\psi \in H_{\mathbb{C}}$. Then,

(a) For any collection $h_{1}, \ldots, h_{M}$ bounded by $\bar{h}$,

$$
\sqrt{T} \boldsymbol{b}_{M}^{(T)} \stackrel{\mathcal{D}}{\rightarrow} \mathcal{N}_{2 M}\left(\mathbf{0}, \Sigma_{0}\right) \quad(T \rightarrow \infty),
$$

where $\stackrel{\mathcal{D}}{\rightarrow}$ denotes convergence in distribution and $\mathcal{N}_{2 M}\left(\mathbf{0}, \Sigma_{0}\right)$ a $2 M$-dimensional normal distribution with mean $\mathbf{0}$ and diagonal covariance matrix $\Sigma_{0}=\operatorname{diag}\left(\sigma_{0, m}^{2}\right.$ : $m=1, \ldots, 2 M)$ whose elements are

$$
\sigma_{0, m}^{2}=\lim _{T \rightarrow \infty} \sum_{l_{i}, l_{i}^{\prime}, l_{2}, l_{2}^{\prime}=1}^{L} T \operatorname{cov}\left(\Re \gamma_{h_{m}}^{(T)}\left(l_{1}, l_{1}^{\prime}\right), \Re \gamma_{h_{m}}^{(T)}\left(l_{2}, l_{2}^{\prime}\right)\right), \quad m=1, \ldots, M
$$

and $\sigma_{0, M+m}^{2}=\sigma_{0, m}^{2}$. The explicit form of the limit is given by Theorem 4.4.4.

(b) Using the result in (a), it follows that

$$
\hat{Q}_{M}^{(T)} \stackrel{\mathcal{D}}{\rightarrow} \chi_{2 M}^{2} \quad(T \rightarrow \infty),
$$

where $\chi_{2 M}^{2}$ a $\chi^{2}$-distributed random variable with $2 M$ degrees of freedom.

The proof of Theorem 4.4.5 is given in Appendix B.4. Part (b) of the theorem can now be used to construct tests with asymptotic level $\alpha$. To better understand the power of the test, the next section investigates the behavior under the alternative of local stationarity.

\subsubsection{Properties under the alternative}

This section contains the counterparts of the results in Section 4.4 .1 for locally stationary functional time series. The following conditions are essential for the large sample results to be established here. 
Assumption 4.4.6. Assume $\left(X_{t}^{(T)}: t \leqslant T, T \in \mathbb{N}\right)$ and $\left(X_{t}^{(u)}: t \in \mathbb{Z}\right)$ are as in Definition 4.3 .1 and let $\kappa_{k ; t_{1}, \ldots, t_{k-1}}$ be a positive sequence in $L^{2}\left([0,1]^{k}, \mathbb{R}\right)$ independent of $T$ such that, for all $j=1, \ldots, k-1$ and some $\ell \in \mathbb{N}$,

$$
\sum_{t_{1}, \ldots, t_{k-1} \in \mathbb{Z}}\left(1+\left|t_{j}\right|^{\ell}\right)\left\|\kappa_{k ; t_{1}, \ldots, t_{k-1}}\right\|_{2}<\infty .
$$

Suppose furthermore that there exist representations

$$
X_{t}^{(T)}-X_{t}^{(t / T)}=Y_{t}^{(T)} \quad \text { and } \quad X_{t}^{(u)}-X_{t}^{(v)}=(u-v) Y_{t}^{(u, v)},
$$

for some processes $\left(Y_{t}^{(T)}: t \leqslant T, T \in \mathbb{N}\right)$ and $\left(Y_{t}^{(u, v)}: t \in \mathbb{Z}\right)$ taking values in $H_{\mathbb{R}}$ whose $k$-th order joint cumulants satisfy

(i) $\left\|\operatorname{cum}\left(X_{t_{1}}^{(T)}, \ldots, X_{t_{k-1}}^{(T)}, Y_{t_{k}}^{(T)}\right)\right\|_{2} \leqslant \frac{1}{T}\left\|\kappa_{k ; t_{1}-t_{k}, \ldots, t_{k-1}-t_{k}}\right\|_{2}$,

(ii) $\left\|\operatorname{cum}\left(X_{t_{1}}^{\left(u_{1}\right)}, \ldots, X_{t_{k-1}}^{\left(u_{k-1}\right)}, Y_{t_{k}}^{\left(u_{k}, v\right)}\right)\right\|_{2} \leqslant\left\|\kappa_{k ; t_{1}-t_{k}, \ldots, t_{k-1}-t_{k}}\right\|_{2}$,

(iii) $\sup _{u}\left\|\operatorname{cum}\left(X_{t_{1}}^{(u)}, \ldots, X_{t_{k-1}}^{(u)}, X_{t_{k}}^{(u)}\right)\right\|_{2} \leqslant\left\|\kappa_{k ; t_{1}-t_{k}, \ldots, t_{k-1}-t_{k}}\right\|_{2}$,

(iv) $\sup _{u}\left\|\frac{\partial^{\ell}}{\partial u^{\ell}} \operatorname{cum}\left(X_{t_{1}}^{(u)}, \ldots, X_{t_{k-1}}^{(u)}, X_{t_{k}}^{(u)}\right)\right\|_{2} \leqslant\left\|\kappa_{k ; t_{1}-t_{k}, \ldots, t_{k-1}-t_{k}}\right\|_{2}$.

Note that these assumptions are generalizations of the ones in Lee and Subba Rao (2016), who investigated the properties of quadratic forms of stochastic processes in a finite-dimensional setting. For fixed $u_{0}$, the process $\left(X_{t}^{\left(u_{0}\right)}: t \in \mathbb{Z}\right)$ is stationary and thus the results of van Delft and Eichler (2016) imply that the local $k$-th order cumulant spectral kernel

$$
f_{u_{0} ; \omega_{1}, \ldots, \omega_{k-1}}\left(\tau_{1}, \ldots, \tau_{k}\right)=\frac{1}{(2 \pi)^{k-1}} \sum_{t_{1}, \ldots, t_{k-1} \in \mathbb{Z}} c_{u_{0} ; t_{1}, \ldots, t_{k-1}}\left(\tau_{1}, \ldots, \tau_{k}\right) e^{-\mathrm{i} \sum_{l=1}^{k-1} \omega_{l} t_{l}}
$$

exists, where $\omega_{1}, \ldots, \omega_{k-1} \in[-\pi, \pi]$ and

$$
c_{u_{0} ; t_{1}, \ldots, t_{k-1}}\left(\tau_{1}, \ldots, \tau_{k}\right)=\operatorname{cum}\left(X_{t_{1}}^{\left(u_{0}\right)}\left(\tau_{1}\right), \ldots, X_{t_{k-1}}^{\left(u_{0}\right)}\left(\tau_{k-1}\right), X_{t_{0}}^{\left(u_{0}\right)}\left(\tau_{k}\right)\right)
$$

is the corresponding local cumulant kernel of order $k$ at time $u_{0}$. The quantity $f_{u, \omega}$ will be referred to as the time-varying spectral density kernel of the stochastic process $\left(X_{t}^{(T)}: t \leqslant T, T \in \mathbb{N}\right)$. This expression is formally justified by Lemma B.1.4.

Because of the standardization necessary in $\hat{\gamma}^{(T)}$, it is of importance to consider the properties of the estimator (4.12) in case the process is locally stationary. The next theorem shows that it is a consistent estimator of the integrated time-varying spectral density operator

$$
G_{\omega}=\int_{0}^{1} \mathcal{F}_{u, \omega} d u
$$

where the convergence is uniform in $\omega \in[-\pi, \pi]$ with respect to $\|\cdot \cdot\|_{2}$.

Theorem 4.4.7 (Consistency and uniform convergence). Suppose $\left(X_{t}^{(T)}: t \leqslant\right.$ $T, T \in \mathbb{N}$ ) satisfies Assumption 4.4 .6 for $\ell=2$ and consider the estimator $\hat{\mathcal{F}}_{\omega}^{(T)}$ in (4.12) with bandwidth fulfilling Assumption 4.3.2. Then, 
(i) $\mathbb{E}\left[\left\|\hat{\mathcal{F}}_{\omega}^{(T)}-G_{\omega}\right\|_{2}^{2}\right]=O\left((b T)^{-1}+b^{4}\right)$;

(ii) $\sup _{\omega \in[-\pi, \pi]}\left\|\hat{\mathcal{F}}_{\omega}^{(T)}-G_{\omega}\right\|_{2} \stackrel{p}{\rightarrow} 0$,

uniformly in $\omega \in[-\pi, \pi]$.

The proof of Theorem 4.4.7 is given in Section B.6 of the Appendix.

To obtain the distributional properties of $\hat{\gamma}^{(T)}$, it is again necessary to replace the denominator with its deterministic limit. In analogy with Theorem 4.4.1, the theorem below gives conditions on the bandwidth for which this is justified under the alternative.

Theorem 4.4.8. Let Assumption 4.4.6 be satisfied with $k=8$ and $\ell=2$ and assume that $\inf _{\omega}\left\langle G_{\omega}(\psi), \psi\right\rangle>0$ for all $\psi \in H_{\mathbb{C}}$. Then,

$$
\sqrt{T}\left|\gamma_{h}^{(T)}-\hat{\gamma}_{h}^{(T)}\right|=O_{p}\left(\frac{1}{\sqrt{b T}}+b^{2}+\frac{1}{b \sqrt{T}}\right) \quad(T \rightarrow \infty) .
$$

The proof of Theorem 4.4.8 is given in Section B.2 of the Appendix. The theorem shows that for $\hat{\gamma}_{h}^{(T)}$ to have the same asymptotic sampling properties as $\gamma_{h}^{(T)}$, additional requirements on the bandwidth $b$ are needed. These are stated next.

Assumption 4.4.9. The bandwidth $b$ satisfies $b \rightarrow 0$ such that $b \sqrt{T} \rightarrow \infty$ as $T \rightarrow \infty$.

The conditions imply that the bandwidth should tend to zero at a slower rate than in the stationary case. The conditions on the bandwidth imposed in this chapter are in particular weaker than the ones used by Dwivedi and Subba Rao (2011) in the finite-dimensional context.

The dependence structure of $\gamma_{h}^{(T)}$ under the alternative is more involved than under the null of stationarity because the mean is nonzero for $h \neq 0 \bmod T$. Additionally, the real and imaginary component of the covariance structure are correlated.

Theorem 4.4.10. Let Assumption 4.4.6 be satisfied with $k=\{2,4\}$. Then, for $h=1, \ldots, T-1$,

$$
\mathbb{E}\left[\gamma_{h}^{(T)}\left(l, l^{\prime}\right)\right]=\frac{1}{2 \pi} \int_{0}^{2 \pi} \int_{0}^{1} \frac{\mathcal{F}_{u, \omega}^{\left(l, l^{\prime}\right)} e^{-\mathrm{i} 2 \pi u h}}{\left(G_{\omega}^{(l, l)} G_{\omega+\omega_{h}}^{\left(l^{\prime}, l^{\prime}\right)}\right)^{1 / 2}} d u d \omega+O\left(\frac{1}{T}\right)=O\left(\frac{1}{h^{2}}\right)+O\left(\frac{1}{T}\right)
$$

The covariance structure satisfies

1. $T \operatorname{cov}\left(\Re \gamma_{h_{1}}^{(T)}\left(l_{1}, l_{2}\right), \Re \gamma_{h_{2}}^{(T)}\left(l_{3}, l_{4}\right)\right)=$

$$
\frac{1}{4}\left[\Sigma_{h_{1}, h_{2}}^{(T)}\left(\boldsymbol{l}_{4}\right)+\dot{\Sigma}_{h_{1}, h_{2}}^{(T)}\left(\boldsymbol{l}_{4}\right)+\grave{\Sigma}_{h_{1}, h_{2}}^{(T)}\left(\boldsymbol{l}_{4}\right)+\bar{\Sigma}_{h_{1}, h_{2}}^{(T)}\left(\boldsymbol{l}_{4}\right)\right]+R_{T},
$$

2. $T \operatorname{cov}\left(\Re \gamma_{h_{1}}^{(T)}\left(l_{1}, l_{2}\right), \Im \gamma_{h_{2}}^{(T)}\left(l_{3}, l_{4}\right)\right)=$

$$
\frac{1}{4 \mathrm{i}}\left[\Sigma_{h_{1}, h_{2}}^{(T)}\left(\boldsymbol{l}_{4}\right)-\dot{\Sigma}_{h_{1}, h_{2}}^{(T)}\left(\boldsymbol{l}_{4}\right)+\grave{\Sigma}_{h_{1}, h_{2}}^{(T)}\left(\boldsymbol{l}_{4}\right)-\bar{\Sigma}_{h_{1}, h_{2}}^{(T)}\left(\boldsymbol{l}_{4}\right)\right]+R_{T},
$$


3. $T \operatorname{cov}\left(\Im \gamma_{h_{1}}^{(T)}\left(l_{1}, l_{2}\right), \Im \gamma_{h_{2}}^{(T)}\left(l_{3}, l_{4}\right)\right)=$

$$
\frac{1}{4}\left[\Sigma_{h_{1}, h_{2}}^{(T)}\left(\boldsymbol{l}_{4}\right)-\dot{\Sigma}_{h_{1}, h_{2}}^{(T)}\left(\boldsymbol{l}_{4}\right)-\grave{\Sigma}_{h_{1}, h_{2}}^{(T)}\left(\boldsymbol{l}_{4}\right)+\bar{\Sigma}_{h_{1}, h_{2}}^{(T)}\left(\boldsymbol{l}_{4}\right)\right]+R_{T},
$$

where $\Sigma_{h_{1}, h_{2}}^{(T)}\left(\boldsymbol{l}_{4}\right), \dot{\Sigma}_{h_{1}, h_{2}}^{(T)}\left(\boldsymbol{l}_{4}\right), \grave{\Sigma}_{h_{1}, h_{2}}^{(T)}\left(\boldsymbol{l}_{4}\right), \bar{\Sigma}_{h_{1}, h_{2}}^{(T)}\left(\boldsymbol{l}_{4}\right)$ are defined in $(4.67)-(4.70)$ and $\left\|R_{T}\right\|_{2}=$ $O\left(T^{-1}\right)$.

The proof of Theorem 4.4.10 is given in Section B.6 of the Appendix. The last result in this section concerns the asymptotic properties of $\hat{Q}_{M}^{(T)}$ in (4.15) under the alternative of local stationarity. Before we state the result, observe that the previous theorem shows that a non-centrality parameter will have to enter since the mean of $\gamma_{h}^{(T)}\left(l, l^{\prime}\right)$ is nonzero. Henceforth, the limit of (4.22) shall be denoted by $\mu_{h}\left(l, l^{\prime}\right)$, i.e.,

$$
\mu_{h}\left(l, l^{\prime}\right)=\frac{1}{2 \pi} \int_{0}^{2 \pi} \int_{0}^{1} \frac{\mathcal{F}_{u, \omega}^{\left(l, l^{\prime}\right)} e^{-\mathrm{i} 2 \pi u h}}{\left(G_{\omega}^{(l, l)} G_{\omega+\omega_{h}}^{\left(l^{\prime}, l^{\prime}\right)}\right)^{1 / 2}} d u d \omega
$$

and its corresponding vectorization by $\boldsymbol{\mu}_{\boldsymbol{h}}$. There is a nice intuitive interpretation of the degree of nonstationarity that can be detected by these functions ${ }^{1}$. For fixed $l, l^{\prime}$ and for small $h$, they can be seen to approximate the Fourier coefficients of the function $\mathcal{F}_{u, \omega}^{\left(l, l^{\prime}\right)} /\left(\mathcal{F}_{\omega}^{(l, l)} \mathcal{F}_{\omega}^{\left(l^{\prime}, l^{\prime}\right)}\right)^{1 / 2}$. More specifically, for $h$ small and $T \rightarrow \infty$, they approximate

$$
\vartheta_{h, j}\left(l, l^{\prime}\right)=\frac{1}{2 \pi} \int_{0}^{2 \pi} \int_{0}^{1} \frac{\mathcal{F}_{u, \omega}^{(l, l)}}{\left(G_{\omega}^{(l, l)} G_{\omega}^{\left(l^{\prime}, l^{\prime}\right)}\right)^{1 / 2}} e^{-\mathrm{i} 2 \pi u h-\mathrm{i} j \omega} d u d \omega,
$$

and thus $\mu_{h}\left(l, l^{\prime}\right) \approx \vartheta_{h, 0}\left(l, l^{\prime}\right)$. Clearly, if the process is weakly stationary then the integrand of these coefficients do not depend on $u$ and we find $\mathcal{F}_{u, \omega}^{(l, l} / G_{\omega}^{(l, l)}=1$. All Fourier coefficients are thus zero except for $\vartheta_{0,0}(l, l)$. For off-diagonal elements, we find coherencies and all coefficients are zero except for $\vartheta_{0, j}\left(l, l^{\prime}\right)$. The mean functions therefore can be seen to reveal long-term nonstationary behavior. Unlike testing methods that are based on segments in the time domain, our test should therefore be able to detect smooth changing behavior in the temporal dependence structure. A precise formulation of the asymptotic properties of the test statistic under $H_{A}$ is given in the next theorem.

Theorem 4.4.11. Let Assumptions 4.3.2 and 4.4.9 be satisfied. Let Assumption 4.4 .6 be satisfied with $k=8$ and $\ell=2$ and assume that $\inf _{\omega}\left\langle\mathcal{F}_{\omega}(\psi), \psi\right\rangle>0$ for all $\psi \in H_{\mathbb{C}}$. Then,

(a) For any collection $h_{1}, \ldots, h_{M}$ bounded by $\bar{h}$,

$$
\sqrt{T} \boldsymbol{b}_{M}^{(T)} \stackrel{\mathcal{D}}{\rightarrow} \mathcal{N}_{2 M}\left(\boldsymbol{\mu}, \Sigma_{A}\right) \quad(T \rightarrow \infty),
$$

where $\mathcal{N}_{2 M}\left(\boldsymbol{\mu}, \Sigma_{A}\right)$ denotes a $2 M$-dimensional normal distribution with mean $\boldsymbol{\mu}=\left(\Re \boldsymbol{e}^{\top} \boldsymbol{\mu}_{\boldsymbol{h}_{\mathbf{1}}}, \ldots, \Re \boldsymbol{e}^{\top} \boldsymbol{\mu}_{\boldsymbol{h}_{M}}, \Im \boldsymbol{e}^{\top} \boldsymbol{\mu}_{\boldsymbol{h}_{\mathbf{1}}}, \ldots, \Im \boldsymbol{e}^{\top} \boldsymbol{\mu}_{\boldsymbol{h}_{M}}\right)^{\top}$ and block covariance matrix

$$
\Sigma_{A}=\left(\begin{array}{cc}
\Sigma_{A}^{(11)} & \Sigma_{A}^{(12)} \\
\Sigma_{A}^{(21)} & \Sigma_{A}^{(22)}
\end{array}\right)
$$

\footnotetext{
${ }^{1}$ See also Paparoditis (2009); Dwivedi and Subba Rao (2011).
} 
whose $M \times M$ blocks are, for $m, m^{\prime}=1, \ldots, M$, given by

$\Sigma_{A}^{(11)}\left(m, m^{\prime}\right)=\lim _{T \rightarrow \infty} \sum_{l_{1}, l_{2}, l_{3}, l_{4}=1}^{L} \frac{1}{4}\left[\Sigma_{h_{m}, h_{m^{\prime}}}^{(T)}\left(\boldsymbol{l}_{4}\right)+\dot{\Sigma}_{h_{m}, h_{m^{\prime}}}^{(T)}\left(\boldsymbol{l}_{4}\right)+\grave{\Sigma}_{h_{m}, h_{m^{\prime}}}^{(T)}\left(\boldsymbol{l}_{4}\right)+\bar{\Sigma}_{h_{m}, h_{m^{\prime}}}^{(T)}\left(\boldsymbol{l}_{4}\right)\right]$,

$\Sigma_{A}^{(12)}\left(m, m^{\prime}\right)=\lim _{T \rightarrow \infty} \sum_{l_{1}, l_{2}, l_{3}, l_{4}=1}^{L} \frac{1}{4 \overline{\bar{i}}}\left[\Sigma_{h_{m}, h_{m^{\prime}}}^{(T)}\left(\boldsymbol{l}_{4}\right)-\dot{\Sigma}_{h_{m}, h_{m^{\prime}}}^{(T)}\left(\boldsymbol{l}_{4}\right)+\grave{\Sigma}_{h_{m}, h_{m^{\prime}}}^{(T)}\left(\boldsymbol{l}_{4}\right)-\bar{\Sigma}_{h_{m}, h_{m^{\prime}}}^{(T)}\left(\boldsymbol{l}_{4}\right)\right]$,

$\Sigma_{A}^{(22)}\left(m, m^{\prime}\right)=\lim _{T \rightarrow \infty} \sum_{l_{1}, l_{2}, l_{3}, l_{4}=1}^{L} \frac{1}{4}\left[\Sigma_{h_{m}, h_{m^{\prime}}}^{(T)}\left(\boldsymbol{l}_{4}\right)-\dot{\Sigma}_{h_{m}, h_{m^{\prime}}}^{(T)}\left(\boldsymbol{l}_{4}\right)-\grave{\Sigma}_{h_{m}, h_{m^{\prime}}}^{(T)}\left(\boldsymbol{l}_{4}\right)+\bar{\Sigma}_{h_{m}, h_{m^{\prime}}}^{(T)}\left(\boldsymbol{l}_{4}\right)\right]$,

where $\Sigma_{h_{m}, h_{m^{\prime}}}^{(T)}\left(\boldsymbol{l}_{4}\right), \dot{\Sigma}_{h_{m}, h_{m^{\prime}}}^{(T)}\left(\boldsymbol{l}_{4}\right), \grave{\Sigma}_{h_{m}, h_{m^{\prime}}}^{(T)}\left(\boldsymbol{l}_{4}\right), \bar{\Sigma}_{h_{m}, h_{m^{\prime}}}^{(T)}\left(\boldsymbol{l}_{4}\right)$ are as in Theorem 4.4.10.

(b) Using the result in (a), it follows that

$$
\hat{Q}_{M}^{(T)} \stackrel{\mathcal{D}}{\rightarrow} \chi_{\mu, 2 M}^{2}, \quad(T \rightarrow \infty),
$$

where $\chi_{\mu, 2 M}^{2}$ denotes a generalized noncentral $\chi^{2}$ random variable with noncentrality parameter $\mu=\|\boldsymbol{\mu}\|_{2}^{2}$ and $2 M$ degrees of freedom.

The proof of Theorem 4.4.11 is given in Appendix B.4.

\subsection{Empirical results}

This section reports the results of an illustrative simulation study designed to verify that the large sample theory is useful for applications to finite samples. The findings provide guidelines for a further fine-tuning of the test procedure, but this will be investigated in detail in future research.

\subsubsection{Simulation setting}

To generate functional time series, the general strategy applied, for example in the papers by Aue et al. (2015) and Hörmann et al. (2015), is utilized. For this simulation study, all processes are build on a Fourier basis representation on the unit interval $[0,1]$ with basis functions $\psi_{1}, \ldots, \psi_{15}$. Note that the $l$-th Fourier coefficient of a $p$-th order functional autoregressive, $\operatorname{FAR}(p)$, process $\left(X_{t}: t \in \mathbb{Z}\right)$ satisfies

$$
\begin{aligned}
\left\langle X_{t}, \psi_{l}\right\rangle & =\sum_{l^{\prime}=1}^{\infty} \sum_{t^{\prime}=1}^{p}\left\langle X_{t-t^{\prime}}, \psi_{l}\right\rangle\left\langle A_{t^{\prime}}\left(\psi_{l}\right), \psi_{l^{\prime}}\right\rangle+\left\langle\varepsilon_{t}, \psi_{l}\right\rangle \\
& \approx \sum_{l^{\prime}=1}^{L_{\max }} \sum_{t^{\prime}=1}^{p}\left\langle X_{t-t^{\prime}}, \psi_{l}\right\rangle\left\langle A_{t^{\prime}}\left(\psi_{l}\right), \psi_{l^{\prime}}\right\rangle+\left\langle\varepsilon_{t}, \psi_{l}\right\rangle,
\end{aligned}
$$

the quality of the approximation depending on the choice of $L_{\max }$. The vector of the first $L_{\max }$ Fourier coefficients $\mathbf{X}_{t}=\left(\left\langle X_{t}, \psi_{1}\right\rangle, \ldots,\left\langle X_{t}, \psi_{L_{\max }}\right\rangle\right)^{\top}$ can thus be generated using the $p$-th order vector autoregressive, $\operatorname{VAR}(p)$, equations

$$
\mathbf{X}_{t}=\sum_{t^{\prime}=1}^{p} \mathbf{A}_{t^{\prime}} \mathbf{X}_{t-t^{\prime}}+\boldsymbol{\varepsilon}_{t}
$$


where the $\left(l, l^{\prime}\right)$ element of $\mathbf{A}_{t^{\prime}}$ is given by $\left\langle A_{t^{\prime}}\left(\psi_{l}\right), \psi_{l^{\prime}}\right\rangle$ and $\varepsilon_{t}=\left(\left\langle\varepsilon_{t}, \psi_{1}\right\rangle, \ldots,\left\langle\varepsilon_{t}, \psi_{L_{\max }}\right\rangle\right)^{\top}$. The entries of the matrices $\mathbf{A}_{t^{\prime}}$ are generated as $\mathcal{N}\left(0, \nu_{l, l^{\prime}}^{\left(t^{\prime}\right)}\right)$ random variables, with the specifications of $\nu_{l, l^{\prime}}$ given below. To ensure stationarity or the existence of a causal solution (see Bosq, 2000; van Delft and Eichler, 2016, for the stationary and locally stationary case, respectively), the norms $\kappa_{t^{\prime}}$ of $\boldsymbol{A}_{t^{\prime}}$ are required to satisfy certain conditions, for example, $\sum_{t^{\prime}=1}^{p}\left\|\boldsymbol{A}_{t^{\prime}}\right\|_{\infty}<1$. The functional white noise, FWN, process is included in (4.23) setting $p=0$.

All simulation experiments were implemented by means of the fda package in $R$ and any result reported in the remainder of this section is based on 1000 simulation runs.

\subsubsection{Finite sample performance under the null}

Under the null hypothesis of stationarity, the following data generating processes, DGPs, were studied:

(a) The FWN variables $\varepsilon_{1}, \ldots, \varepsilon_{T}$ with coefficient variances $\operatorname{Var}\left(\left\langle\varepsilon_{t}, \psi_{l}\right\rangle\right)=\exp ((l-$ 1)/10);

(b) The $\operatorname{FAR}(2)$ variables $X_{1}, \ldots, X_{T}$ with operators specified through the respective variances $\nu_{l, l^{\prime}}^{(1)}=\exp \left(-l-l^{\prime}\right)$ and $\nu_{l, l^{\prime}}^{(2)}=1 /\left(l+l^{\prime 3 / 2}\right)$ and norms $\kappa_{1}=0.75$ and $\kappa_{2}=-0.4$, and innovations $\varepsilon_{1}, \ldots, \varepsilon_{T}$ as in (a);

(c) The FAR(2) variables $X_{1}, \ldots, X_{T}$ as in (b) but with $\kappa_{1}=0.4$ and $\kappa_{2}=0.45$.

The sample sizes under consideration are $T=2^{n}$ for $n=6, \ldots, 11$, so that the smallest sample size consists of 64 functions and the largest of 2048 . The test statistic $\hat{Q}_{M}^{(T)}$ in (4.15) is then set up with $L=L_{\max }$ and $h_{m}=m$ for $m=1, \ldots, M$ with $M=1,5$ and 10. A rejection is reported if the simulated test statistic value exceeds the critical level prescribed in part (b) of Theorem 4.4.5. The processes in (a)-(c) comprise a range of stationary scenarios. DGP (a) is the most simple model, specifying an independent FWN process. DGPs (b) and (c) exhibit second-order autoregressive dynamics of different persistence, with the process in (c) possessing the stronger temporal dependence.

The empirical rejection levels for the processes (a)-(c) can be found in Table 4.1. It can be seen that the empirical levels for the statistics $\hat{Q}_{1}^{(T)}$ are generally well adjusted with the notable exception of the larger samples sizes for DGP (c). Here the performance is somewhat conservative, perhaps owing to the high persistence in the underlying DGP. The performance for $\hat{Q}_{5}^{(T)}$ and $\hat{Q}_{10}^{(T)}$ shows additional features. First, rejection levels tend to be too high for smaller sample sizes and too low for larger sample sizes. The former has to do with the degrees of freedom available for the estimation, the latter points to a fact discussed further in Section 4.5.4 below, namely that pooling across too many fDFTs (that is, too large a value of $M$ ) does not aggregate additional information. Some evidence on closeness between empirical and limit densities is provided in Figure 4.1. 

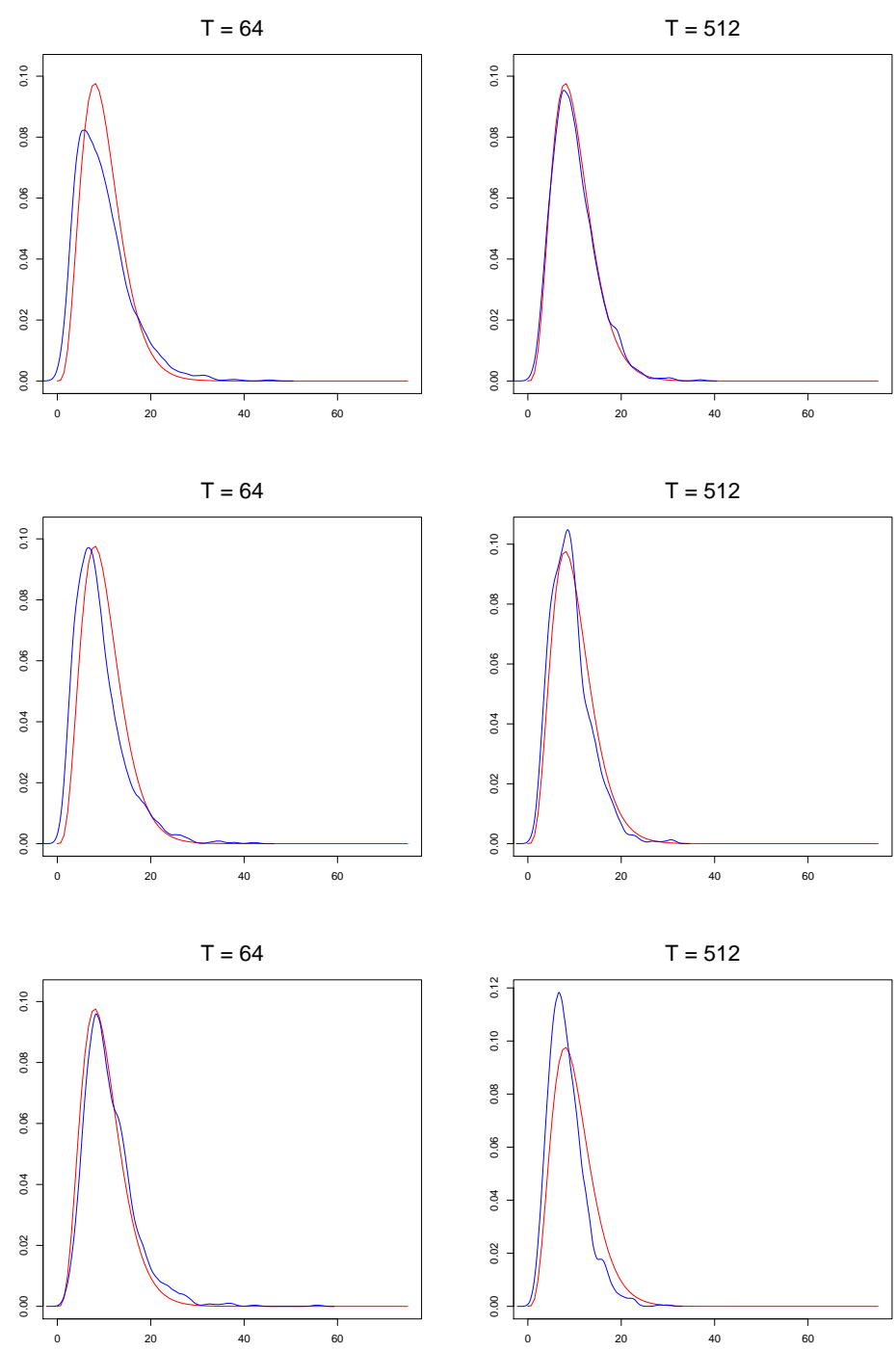

Figure 4.1: Blue: Empirical density of $\hat{Q}_{5}^{(T)}$ for $T=64$ (left panel) and $T=512$ (right panel) for DGPs (a)-(c) (top to bottom). Red: The corresponding chisquared densities predicted under the null. 


\begin{tabular}{rrrllllllll}
\hline & \multicolumn{9}{c}{ \% level } & \multicolumn{3}{c}{ \% level } & \multicolumn{3}{c}{ \% level } \\
& \multicolumn{1}{c}{$T$} & $\hat{Q}_{1}^{(T)}$ & 5 & 1 & $\hat{Q}_{5}^{(T)}$ & 5 & 1 & $\hat{Q}_{10}^{(T)}$ & 5 & 1 \\
\hline (a) & 64 & 1.90 & 4.60 & 0.60 & 9.75 & 8.10 & 2.90 & 19.43 & 11.20 & 6.00 \\
& 128 & 2.05 & 6.40 & 1.60 & 9.85 & 7.30 & 2.70 & 19.95 & 10.40 & 3.80 \\
& 256 & 1.98 & 4.70 & 1.30 & 10.11 & 7.20 & 2.20 & 19.97 & 7.80 & 2.80 \\
& 512 & 2.06 & 5.50 & 1.00 & 10.03 & 6.40 & 1.20 & 20.07 & 6.80 & 2.20 \\
& 1024 & 2.00 & 6.00 & 1.00 & 9.98 & 4.40 & 1.40 & 19.75 & 5.20 & 1.70 \\
& 2048 & 2.04 & 5.50 & 1.20 & 10.07 & 5.60 & 0.70 & 20.23 & 4.30 & 1.00 \\
\hline (b) & 64 & 1.78 & 4.00 & 0.90 & 8.94 & 6.10 & 2.00 & 17.73 & 8.40 & 4.70 \\
& 128 & 1.93 & 5.00 & 1.70 & 9.01 & 4.90 & 1.70 & 18.01 & 6.00 & 2.00 \\
& 256 & 1.83 & 4.10 & 1.20 & 9.26 & 5.40 & 0.70 & 18.10 & 5.10 & 1.70 \\
& 512 & 1.88 & 4.00 & 0.70 & 9.19 & 3.70 & 0.90 & 18.31 & 3.40 & 0.40 \\
& 1024 & 1.83 & 4.20 & 0.60 & 9.15 & 3.30 & 0.60 & 18.08 & 3.00 & 1.40 \\
& 2048 & 1.87 & 5.00 & 0.60 & 9.21 & 3.20 & 0.20 & 18.49 & 1.90 & 0.40 \\
\hline (c) & 64 & 2.10 & 5.80 & 0.80 & 11.17 & 8.80 & 3.40 & 23.64 & 16.10 & 8.10 \\
& 128 & 1.75 & 4.00 & 0.80 & 8.84 & 4.00 & 0.50 & 17.60 & 4.30 & 1.40 \\
& 256 & 1.94 & 5.10 & 1.20 & 9.41 & 5.90 & 1.60 & 19.79 & 7.90 & 3.10 \\
& 512 & 1.73 & 3.30 & 0.60 & 8.36 & 2.20 & 0.20 & 16.80 & 2.30 & 0.10 \\
& 1024 & 1.62 & 2.70 & 0.20 & 8.19 & 1.40 & 0.20 & 16.22 & 1.90 & 0.40 \\
& 2048 & 1.66 & 3.00 & 0.40 & 8.23 & 1.70 & 0.10 & 16.49 & 0.70 & 0.20 \\
\hline
\end{tabular}

Table 4.1: Averaged test statistic values and rejection rates of $\hat{Q}_{M}^{(T)}$ at the $1 \%$ and $5 \%$ asymptotic level for the processes (a)-(c) for various choices of $M$ and $T$. All table entries are generated from 1000 repetitions.

\subsubsection{Finite sample performance under the alternative}

Under the alternative, the following data generating processes are considered:

(d) The tvFAR(1) variables $X_{1}, \ldots, X_{T}$ with operator specified through the variances $\nu_{l, l^{\prime}}^{(1)}=\exp \left(-l-l^{\prime}\right)$ and norm $\kappa_{1}=0.8$, and innovations given by (a) with added multiplicative time-varying variance

$$
\sigma^{2}(t)=\frac{1}{2}+\cos \left(\frac{2 \pi t}{2048}\right)+0.3 \sin \left(\frac{2 \pi t}{2048}\right)
$$

(e) The $\operatorname{tvFAR}(2)$ variables $X_{1}, \ldots, X_{T}$ with both operators as in (d) but with time-varying norm

$$
\kappa_{1, t}=1.8 \cos \left(1.5-\cos \left(\frac{4 \pi t}{T}\right)\right),
$$

constant norm $\kappa_{2}=-0.81$, and innovations as in (a);

(f) The structural break $\operatorname{FAR}(2)$ variables $X_{1}, \ldots, X_{T}$ given in the following way. 
- For $t \leqslant 3 T / 8$, the operators are as in (b) but with norms $\kappa_{1}=0.7$ and $\kappa_{2}=0.2$, and innovations as in (a);

- For $t>3 T / 8$, the operators are as in (b) but with norms $\kappa_{1}=0$ and $\kappa_{2}=-0.2$, and innovations as in (a) but with variances $\operatorname{Var}\left(\left\langle\varepsilon_{t}, \psi_{l}\right\rangle\right)=$ $2 \exp ((l-1) / 10)$.

All other aspects of the simulations are as in Section 4.5.1. The processes studied under the alternative provide intuition for the behavior of the proposed test under different deviations from the null hypothesis. DGP (d) is time-varying only through the innovation structure, in the form of a slowly varying variance component. The first-order autoregressive structure is independent of time. DGP (e) is a time-varying second-order FAR process for which the first autoregressive operator varies with time. The final DGP in (f) models a structural break, a different type of alternative. Here, the process is not locally stationary as prescribed under the alternative in this chapter, but piecewise stationary with the two pieces being specified as two distinct $\mathrm{FAR}(2)$ processes.

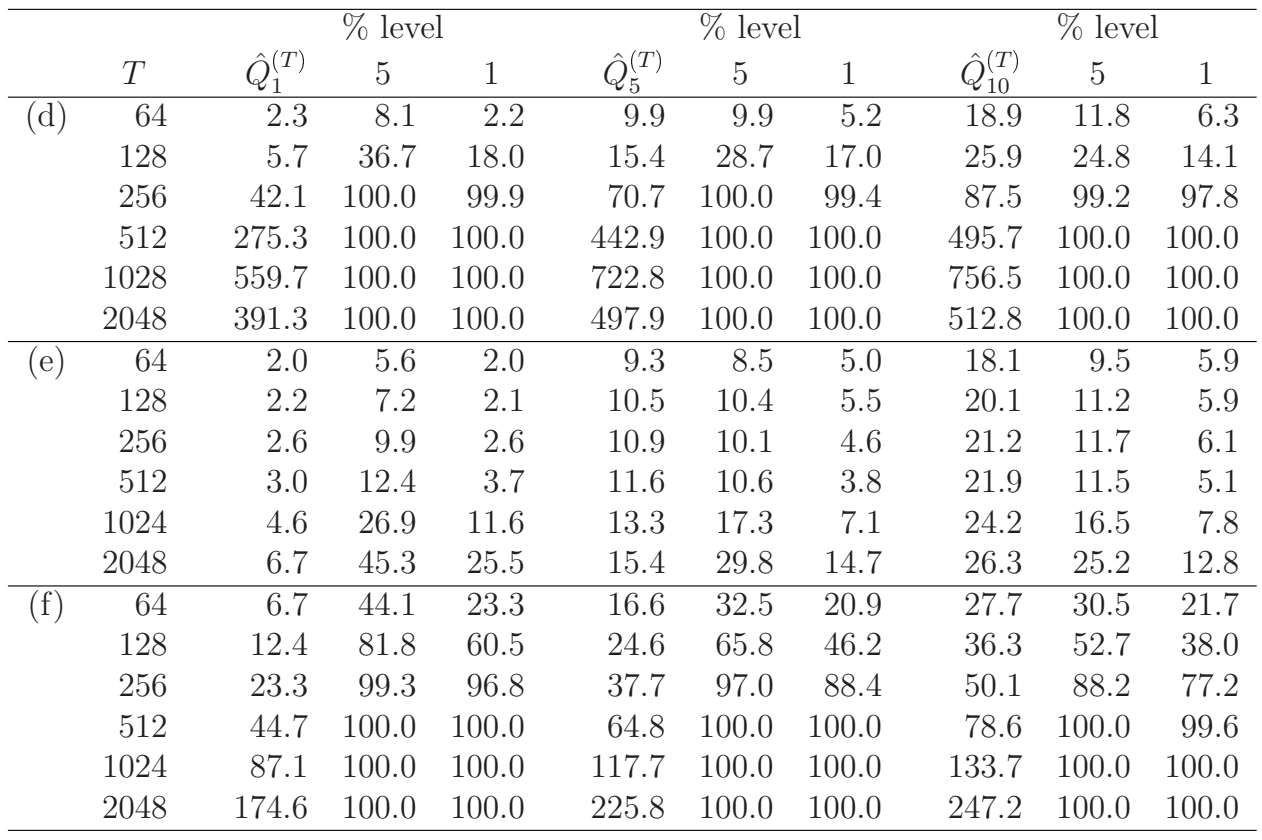

Table 4.2: Averaged test statistic values and rejection rates of $\hat{Q}_{M}^{(T)}$ at the $1 \%$ and $5 \%$ asymptotic level for the processes (d)-(f) for various choices of $M$ and $T$. All table entries are generated from 1000 repetitions.

The empirical power of the various test statistics for the processes in (d)-(f) are in Table 4.2. Power results are roughly similar across the selected values of $M$. For 
DGP (d), power is low for the small sample sizes $T=64$ and 128 and is at $100 \%$ for the all larger $T$. The low power is explained by the form of the time-varying variance which takes 2048 observations to complete a full cycle of the sine and cosine components. In the situation of the two smallest sample sizes, this slowly varying variance appears close to stationary and therefore rejections of the null are rare. Closeness of the distributions under the null hypothesis and the alternative can be measured in part by the size of the non-centrality parameter $\boldsymbol{\mu}$. Table 4.4 collects information on the size. It can be seen from the top two panels that for DGP (d), the corresponding real and imaginary parts, and the squared norm are small for the small sample size $T=6$. If the sample size is increased to $T=512$, these values are much larger, in particular for small choices of $h$.

DGP (e) shows low power throughout. Again, the reason for this is that the form of local stationarity under consideration here resembles too closely stationary behavior expected under the null hypothesis. This is corroborated in Figure 4.2, where the empirical version of the non-central generalized chi-squared limit under the alternative is closely aligned with the standard chi-squared limit expected under the null hypothesis. The corresponding estimates of $\boldsymbol{\mu}$ remain small even for $T=512$ as shown in the third panel of Table 4.4.

The results for DGP (f) indicate that the proposed statistics has power against structural break alternatives. This statement is supported by Figure 4.2 and the estimated non-centrality parameter size given in Table 4.4 .

\subsubsection{Toward an automated test procedure}

As indicated in Section 4.5.2, a further analysis of the simulation results may provide the seed for the development of a selection procedure for $M$. Some ideas are put forward in this section, but details will be left to future research. A crucial ingredient for the selection of $M$ and the corresponding frequencies $h_{m}$ (in the simulations $h_{m}=m$ but other choices can be entertained) is the size of the various $\hat{\boldsymbol{\beta}}_{h_{m}}^{(T)}$ in (4.14) whose real and imaginary part make up the vector $\hat{\mathbf{b}}_{M}^{(T)}$ in the test statistics $\hat{Q}_{M}^{(T)}$. Computing the values $\left|\hat{\boldsymbol{\beta}}_{h_{m}}^{(T)}\right|^{2}$ over $m=1, \ldots, M$ shows that these values are uniformly small across both $m$ and sample sizes $T$ under the null of stationarity of the underlying functional time series. Under the alternative of local stationarity (as well as in the structural break scenario), several $\left|\hat{\boldsymbol{\beta}}_{h_{m}}^{(T)}\right|^{2}$ are comparatively large. These $m$ should be chosen by a selection procedure, for example through a thresholding approach to be developed. For illustration purposes, Table 4.3 shows the size of $\left|\hat{\boldsymbol{\beta}}_{h_{m}}^{(T)}\right|^{2}$ across a range of options considered in the simulations. The general observation deduced from running a multitude of simulations under both null and alternative scenarios is that the $\hat{\boldsymbol{\beta}}_{h_{m}}^{(T)}$ appear to decay faster for the nonstationary processes under considerations, and that they are rather constant under stationarity.

Figure 4.3 provides contour plots of the modulus of $\hat{\gamma}_{1}^{(T)}$ for model (b) under the null and for models (d)-(f) under the alternative. The contours are obtained from averaging (over the simulation runs) the aggregated contributions $\hat{\gamma}_{1}^{(T)}\left(l, l^{\prime}\right)$ and evaluating these contributions in the direction of $\psi_{l} \otimes \psi_{l^{\prime}}$ for $l, l^{\prime}=1, \ldots, L$. It 
can be seen that the magnitude of the contours provides another indicator for how easy or hard it may be to reject the null hypothesis. The top row in the figure is for the stationary DGP (b). For any of the sample sizes considered, the magnitudes across $[0,1]^{2}$ remains small, as expected under the null. The behavior under the alternative is markedly different, but the specifics depend on the type of alternative. For the time-varying noise process $(\mathrm{d})$, the contribution of nonstationarity is at the diagonal, with the magnitude along this ridge increasing significantly with the sample size. For DGP (e), the form of nonstationarity creates very different contours. The structural break process (f) induces nonstationarity in the contours in a similar way as DGP (d), with strong concentration occurring at the diagonal for all sample sizes. Any future refinement of the test will have to take these features into account. 

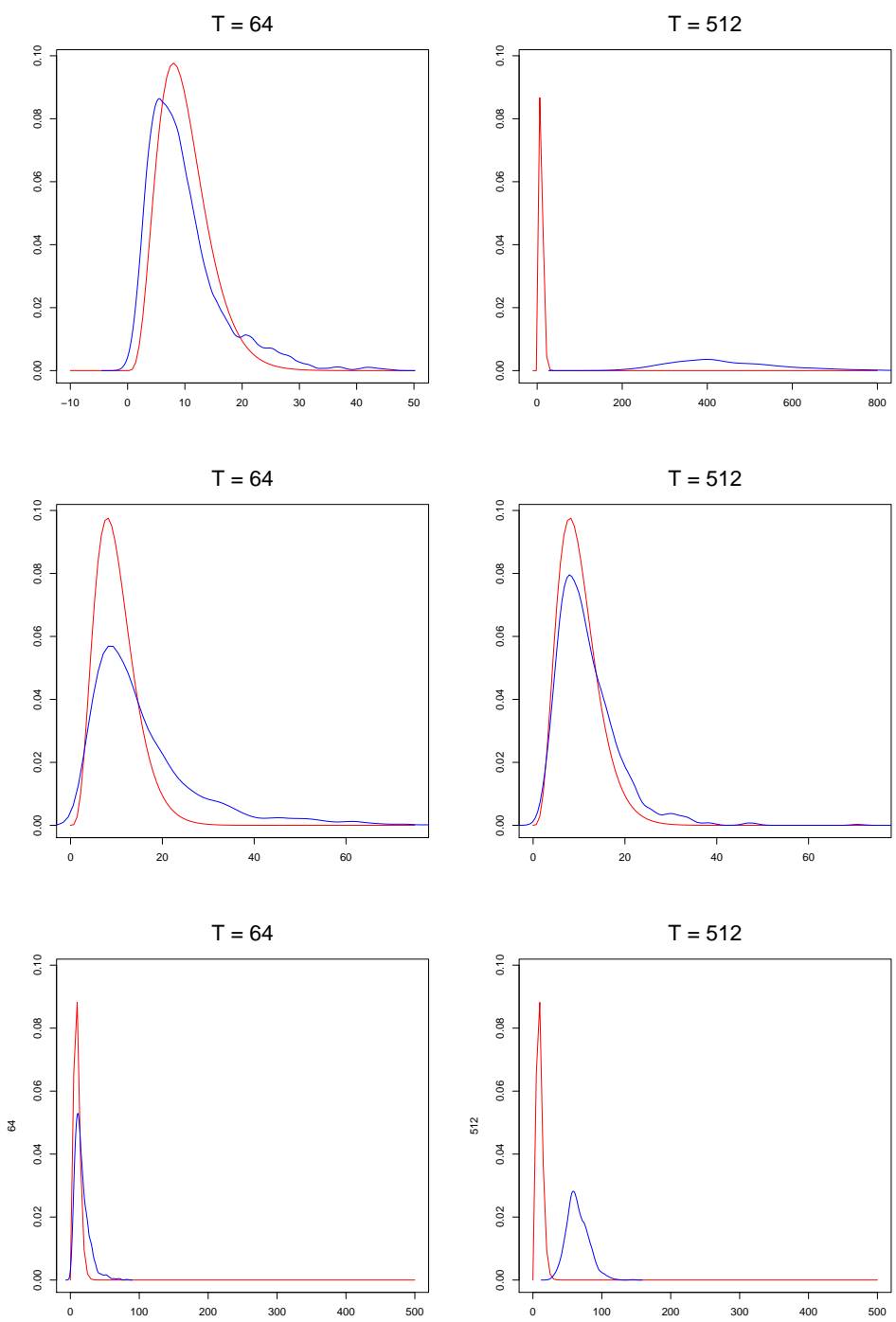

Figure 4.2: Blue: Empirical density of $\hat{Q}_{5}^{(T)}$ for $T=64$ (left panel) and $T=512$ (right panel) for DGPs (d)-(f) (top to bottom). Red: The corresponding chi-squared densities predicted under the null. 


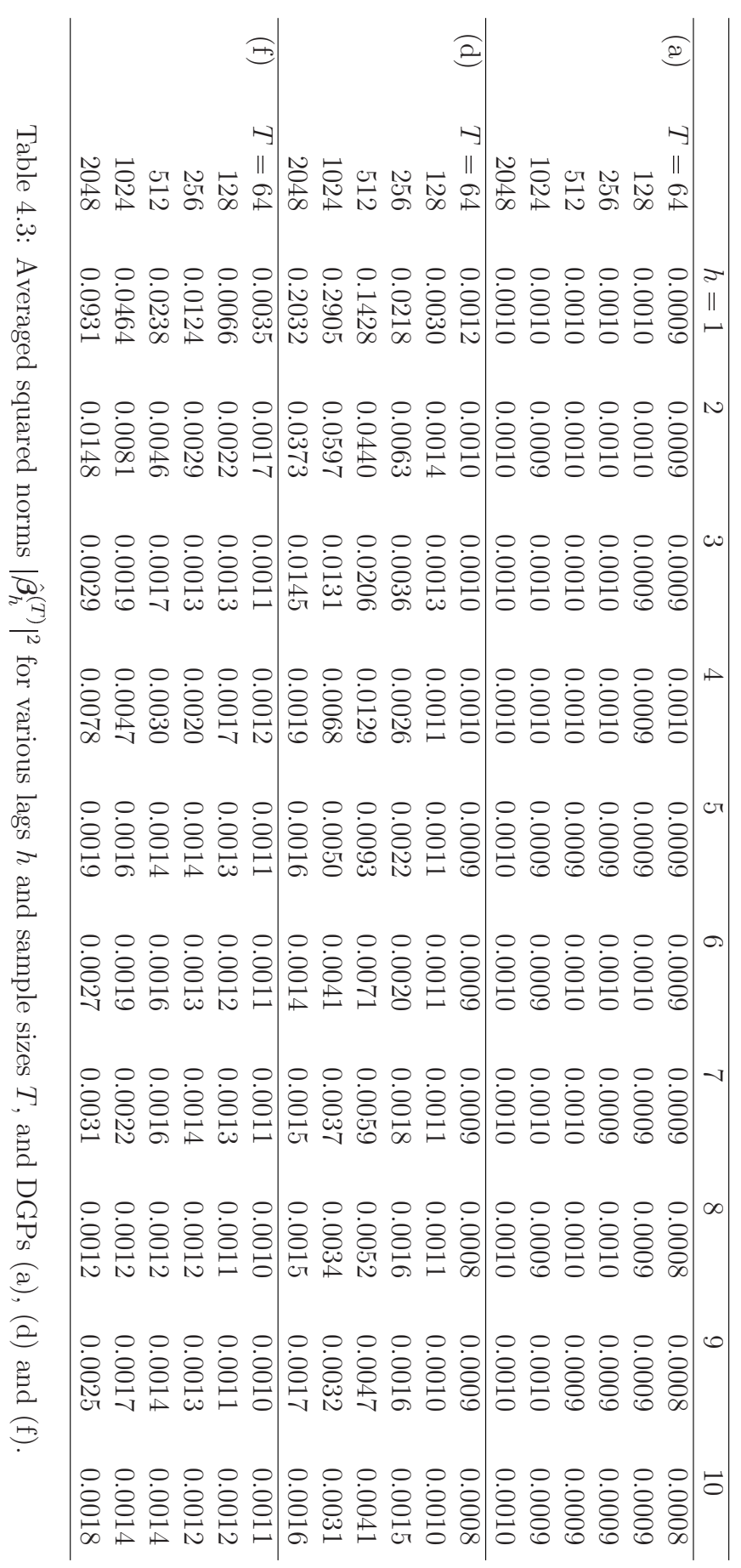




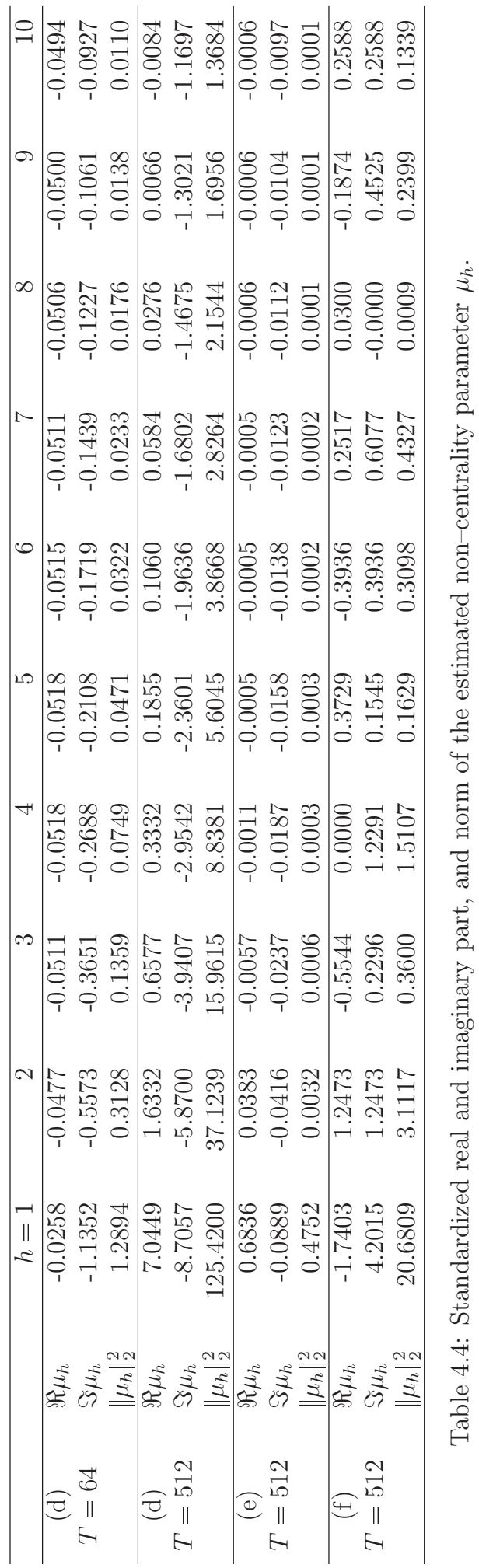



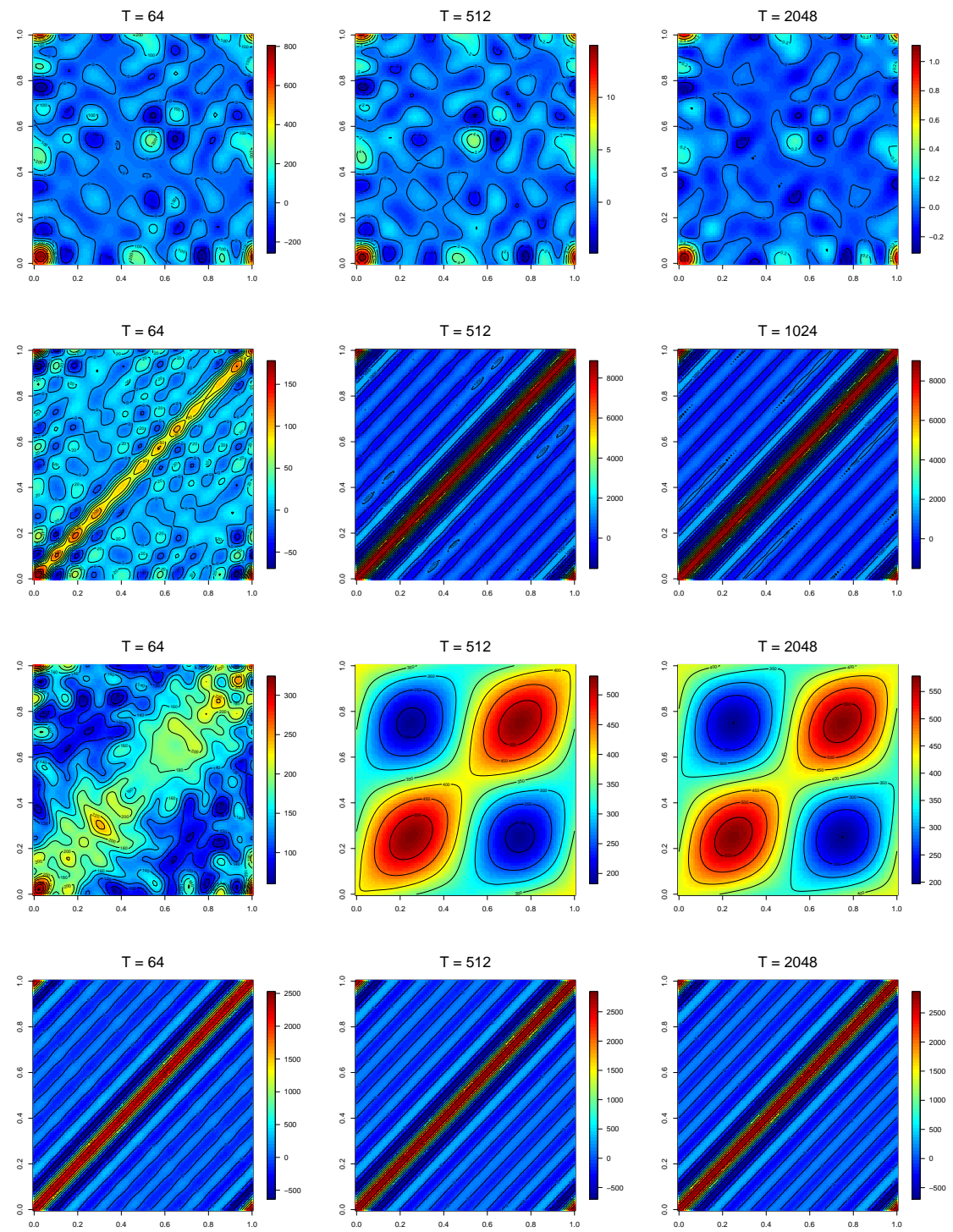

Figure 4.3: Contour plots of $\hat{\gamma}_{1}^{(T)}$ for various sample sizes $T$ and DGPs (b) and (d)-(f) (top to bottom). 


\subsection{Conclusion}

This chapter was concerned with the development of methodology for testing the stationarity of a functional time series. The proposed method is frequency domainbased and exploits that the functional Discrete Fourier Transforms evaluated at distinct canonical frequencies are uncorrelated if and only if the underlying functional time series are weakly stationary. The testing procedure is based on the empirical covariance operator of the functional Discrete Fourier Transforms. The sampling properties of this operator were investigated in detail. No structural modeling assumptions are imposed except for some weak mixing conditions in the form of functional generalizations of classical cumulant mixing conditions. To make the test feasible in practice, some form of dimension reduction is necessary and projected versions were considered. The final test statistic has a quadratic form and its limit distribution was derived to be chi-square under both the null hypothesis of weak stationarity as well as under the alternative of local stationarity.

There is one very important aspect which allows for a thorough investigation of the properties of the test under the alternative of local stationarity. Namely, the inverse Fourier transform of the time-varying spectral density operator evaluated at the canonical frequencies constitutes a sequence of Fourier coefficients of the timevarying spectral density operator. The rate at which these coefficients decay, in an $L^{2}$ sense, is inversely proportional to their absolute distance from the 0-th Fourier coefficient. The 0 -th Fourier coefficient corresponds to the integrated time-varying spectral density operator over all time. A smoothing window applied to the tensor product of the functional Discrete Fourier transform was shown to yield a consistent estimator of the integrated time-varying spectral density operator.

Under the alternative of local stationarity, the asymptotic distribution is a noncentral generalized chi-square random variable. Knowledge on the sampling properties under the alternative of local stationarity promises to be especially useful in further understanding how different 'degrees' of nonstationarity affect both the localization and skewness of the distribution. The expression of the mean function, which largely affects the non-centrality parameter, provides meaningful intuition on the degree of nonstationarity and can be seen as a measure of long-term nonstationary behavior. It is this interesting feature that allows the test statistic to detect very slow and gradual changes. The test is therefore expected to perform better under smooth alternatives than time domain-based testing procedures that rely on dividing the data into segments. One would moreover expect that this feature enables our test to be more robust when the observation frequency low. Smooth functions contain mainly low frequency information, while high frequency fluctuations are more common for non-smooth functions. Intuitively, when the spectral characteristics change very gradually, relatively more observations will be necessary to capture the nonstationary behavior. The precise implications of sparse observations and the degree of nonstationarity that can be detected and a possible quantification of this relationship are left for future research.

Finite sample properties were highlighted in simulation experiments with various data generating processes, including processes that were subject to structural breaks. 
The empirical results show promise for applications to real data. Future research will be devoted to a more in-depth analysis of practical relevance and to actual data analysis. 


\section{Appendix}

\section{B.1 Properties of functional cumulants}

For random elements $X_{1}, \ldots, X_{k}$ in a Hilbert space $H$, the moment tensor of order $k$ can be defined as

$$
\mathbb{E}\left[X_{1} \otimes \cdots \otimes X_{k}\right]=\sum_{l_{1}, \ldots l_{k} \in \mathbb{N}} \mathbb{E}\left[\prod_{t=1}^{k}\left\langle X_{t}, \psi_{l_{t}}\right\rangle\right]\left(\psi_{l_{1}} \otimes \cdots \otimes \psi_{l_{k}}\right),
$$

where the elementary tensors $\left(\psi_{l_{1}} \otimes \cdots \otimes \psi_{l_{k}}: l_{1}, \ldots, l_{k} \in \mathbb{N}\right)$ form an orthonormal basis in the tensor product space $\bigotimes_{j=1}^{k} H$. The latter follows since $\left(\psi_{l}: l \in \mathbb{N}\right)$ is an orthonormal basis of the separable Hilbert space $H$. Similarly, define the $k$-th order cumulant tensor by

$$
\operatorname{cum}\left(X_{1} \otimes \cdots \otimes X_{k}\right)=\sum_{l_{1}, \ldots l_{k} \in \mathbb{N}} \operatorname{cum}\left(\prod_{t=1}^{k}\left\langle X_{t}, \psi_{l_{t}}\right\rangle\right)\left(\psi_{l_{1}} \otimes \cdots \otimes \psi_{l_{k}}\right)
$$

where the cumulants on the right-hand side are as usual given by

$$
\operatorname{cum}\left(\left\langle X_{1}, \psi_{l_{1}}\right\rangle, \ldots,\left\langle X_{k}, \psi_{l_{k}}\right\rangle\right)=\sum_{\nu=\left(\nu_{1}, \ldots, \nu_{p}\right)}(-1)^{p-1}(p-1) ! \prod_{r=1}^{p} \mathbb{E}\left[\prod_{t \in \nu_{r}}\left\langle X_{t}, \psi_{l_{t}}\right\rangle\right] .
$$

where the summation extends over all unordered partitions $\nu$ of $\{1, \ldots, k\}$. The following is a generalization of the product theorem for cumulants (Brillinger, 1981, Theorem 2.3.2).

Theorem B.1.1. Consider the tensor $X_{t}=\otimes_{j=1}^{J_{t}} X_{t j}$ for random elements $X_{t j}$ in $H$ with $j=1, \ldots, J_{t}$ and $t=1, \ldots, k$. Let $\nu=\left\{\nu_{1}, \ldots \nu_{p}\right\}$ be a partition of $\{1, \ldots, k\}$. The joint cumulant tensor $\operatorname{cum}\left(X_{1}, \ldots, X_{k}\right)$ is given by

$\operatorname{cum}\left(X_{1}, \ldots, X_{k}\right)=\sum_{r_{11}, \ldots, r_{k J}} \sum_{\nu=\left(\nu_{1}, \ldots, \nu_{p}\right)} \prod_{n=1}^{p} \operatorname{cum}\left(\left\langle X_{t j}, \psi_{r_{t j}}\right\rangle \mid(t, j) \in \nu_{n}\right) \psi_{r_{11}} \otimes \cdots \otimes \psi_{r_{k J}}$,

where the summation extends over all indecomposable partitions $\nu=\left(\nu_{1}, \ldots, \nu_{p}\right)$ of the table

$$
\begin{array}{ccc}
(1,1) & \cdots & \left(1, J_{1}\right) \\
\vdots & \ddots & \vdots \\
(k, 1) & \cdots & \left(k, J_{t}\right) .
\end{array}
$$


Formally, abbreviate this by

$$
\operatorname{cum}\left(X_{1}, \ldots, X_{k}\right)=\sum_{\nu=\left(\nu_{1}, \ldots, \nu_{p}\right)} S_{\nu}\left(\otimes_{n=1}^{p} \operatorname{cum}\left(X_{t j} \mid(t, j) \in \nu_{n}\right)\right)
$$

where $S_{\nu}$ is the permutation that maps the components of the tensor back into the original order, i.e., $S_{\nu}\left(\otimes_{r=1}^{p} \otimes_{(t, j) \in \nu_{r}} X_{t j}\right)=X_{11} \otimes \cdots \otimes X_{k J_{t}}$.

Next, results for cumulants of the fDFT are stated under both stationarity and local stationarity regimes.

Lemma B.1.2 (Cumulants of the fDFT under stationarity). Let $\left(X_{t}: t \in \mathbb{Z}\right)$ be a $k$-th order stationary sequence taking values in $H_{\mathbb{R}}$ that satisfies Assumption 4.4.1. The cumulant operator of the fDFT then satisfies

$$
\operatorname{cum}\left(D_{\omega_{j_{1}}}^{(T)}, \ldots, D_{\omega_{j_{k}}}^{(T)}\right)=\frac{(2 \pi)^{k / 2-1}}{T^{k / 2}} \Delta_{T}^{\left(\sum_{l=1}^{k} \omega_{j_{l}}\right)} \mathcal{F}_{\omega_{j_{1}}, \ldots, \omega_{j_{k-1}}}+R_{T, k}
$$

where the function $\Delta_{T}^{(\omega)}=T$ for $\omega \equiv 0 \bmod 2 \pi, \Delta_{T}^{\left(\omega_{k}\right)}=0$ for $k \neq 0 \bmod T$ and the remainder satisfies $\left\|R_{T, k}\right\|_{2}=O\left(T^{-k / 2}\right)$.

The proof of this lemma can be found in Panaretos and Tavakoli (2013b). To give the analog of Lemma B.1.2 in the locally stationary case, a number of auxiliary statements will be made first.

Lemma B.1.3. Let Assumption 4.4 .6 be satisfied and $c_{u ; t_{1}, \ldots, t_{k-1}}$ as given in (4.21). Then,

$$
\begin{gathered}
\left\|\operatorname{cum}\left(X_{t_{1}}^{(T)}, \ldots, X_{t_{k-1}}^{(T)}, X_{t_{k}}^{(T)}\right)-c_{t_{1} / T ; t_{1}-t_{k}, \ldots, t_{k-1}-t_{k}}\right\|_{2} \\
\leqslant\left(\frac{k}{T}+\sum_{j=1}^{k-1}\left|\frac{t_{j}-t_{k}}{T}\right|\right)\left\|\kappa_{k ; t_{1}-t_{k}, \ldots, t_{k-1}-t_{k}}\right\|_{2} .
\end{gathered}
$$

Proof. By linearity of the cumulant operation, consecutively taking differences leads by (4.19) and the triangle inequality to

$$
\begin{aligned}
& \left\|\operatorname{cum}\left(X_{t_{1}}^{(T)}, \ldots, X_{t_{k}}^{(T)}\right)-\operatorname{cum}\left(X_{t_{1}}^{\left(t_{1} / T\right)}, \ldots, X_{t_{k}}^{\left(t_{k} / T\right)}\right)\right\|_{2} \\
& =\left\|\sum_{j=1}^{k} \operatorname{cum}\left(X_{t_{1}}^{(T)}, \ldots, Y_{t_{j}}^{(T)}, \ldots, X_{t_{k}}^{(T)}\right)\right\|_{2} \\
& \leqslant K \frac{k}{T}\left\|\kappa_{k ; t_{1}-t_{k}, \ldots, t_{k-1}-t_{k}}\right\|_{2},
\end{aligned}
$$

where we used part (i) of Assumption 4.4.6. By (4.19),

$$
X_{t_{j}}^{\left(t_{j} / T\right)}-X_{t_{j}}^{\left(t_{k} / T\right)}=\frac{\left(t_{j}-t_{k}\right)}{T} Y_{t_{j}}^{\left(t_{j} / T, t_{k} / T\right)} .
$$


Similarly,

$$
\begin{gathered}
\left\|\operatorname{cum}\left(X_{t_{1}}^{\left(t_{1} / T\right)}, \ldots, X_{t_{k}}^{\left(t_{k} / T\right)}\right)-c_{t_{1} / T ; t_{1}-t_{k}, \ldots, t_{k-1}-t_{k}}\right\|_{2} \\
\leqslant \sum_{j=1}^{k-1} \frac{\left|t_{j}-t_{k}\right|}{T}\left\|\kappa_{k ; t_{1}-t_{k}, \ldots, t_{k-1}-t_{k}}\right\|_{2},
\end{gathered}
$$

which follows from part (iii) of Assumption 4.4.6. Minkowski's Inequality then implies the lemma.

Lemma B.1.4. Consider a sequence of functional processes $\left(X_{t}^{(T)}: t \leqslant T, T \in \mathbb{N}\right)$ satisfying Assumption 4.4.6 with $k=2$ and $\ell=2$. Then the triangular array uniquely characterizes the time-varying local spectral density operator

$$
\mathcal{F}_{u, \omega}=\frac{1}{2 \pi} \sum_{h \in \mathbb{Z}} \mathcal{C}_{u, h} e^{-\mathrm{i} \omega h},
$$

which belongs to $S_{2}(H)$ and its kernel satisfies

(i) $\sup _{u, \omega}\left\|\frac{\partial^{i}}{\partial u^{i}} f_{u, \omega}\right\|_{2}<\infty$ for $i=1,2$,

(ii) $\sup _{u, \omega}\left\|\frac{\partial^{i}}{\partial{ }^{i} \omega} f_{u, \omega}\right\|_{2}<\infty$ for $i=1,2$,

(iii) $\sup _{u, \omega}\left\|\frac{\partial^{2}}{\partial \omega \partial u} f_{u, \omega}\right\|_{2}<\infty$.

Proof. Using Lemma B.1.3, it is straightforward to show that the triangular array $\left(X_{t}^{T}: t \leqslant T, T \in \mathbb{N}\right)$ uniquely determines the time-varying spectral density operator, that is,

$$
\int_{-\pi}^{\pi}\left\|\mathcal{F}_{u, \omega}^{(T)}-\mathcal{F}_{u, \omega}\right\|_{2}^{2} d \omega=o(1) \quad(T \rightarrow \infty) .
$$

Existence of the derivatives follow from the Dominated Convergence Theorem and the product rule for differentiation in Banach spaces (Nelson, 1969).

The cumulants of the local fDFT can be expressed in terms of the time-varying spectral operator. At the Fourier Frequencies, the time-varying spectral operator can be shown to possess a well-defined Fourier transform. The properties of the resulting Fourier coefficients make apparent that the dependence structure of the local fDFT behaves in a very specific manner that is based on the distance of the frequencies. The coefficients additionally provide an upper bound on the norm of the cumulant operator. This is summarized in the next lemma.

Lemma B.1.5. Suppose Assumption 4.4.6 holds true. Then the cumulant tensor of the local fDFT satisfies

$$
\begin{aligned}
\operatorname{cum}\left(D_{\omega_{j_{1}}}^{(T)}, \ldots, D_{\omega_{j_{k}}}^{(T)}\right) & =\frac{(2 \pi)^{k / 2-1}}{T^{k / 2}} \sum_{t=0}^{T-1} \mathcal{F}_{t / T ; \omega_{j_{1}}, \ldots, \omega_{j_{k-1}}} e^{-\mathrm{i} \sum_{l=1}^{k} t \omega_{j_{l}}}+R_{k, T} \\
& =\frac{(2 \pi)^{k / 2-1}}{T^{k / 2-1}} \tilde{\mathcal{F}}_{j_{1}+\ldots+j_{k} ; \omega_{j_{1}}, \ldots, \omega_{j_{k-1}}}+R_{k, T}
\end{aligned}
$$


where $\left\|R_{k, T}\right\|_{2}=O\left(T^{-k / 2}\right)$ and the operator

$$
\tilde{\mathcal{F}}_{s ; \omega_{j_{1}}, \ldots, \omega_{j_{k-1}}}=\int_{0}^{1} \mathcal{F}_{u ; \omega_{j_{1}}, \ldots, \omega_{j_{k-1}}} e^{-\mathrm{i} 2 \pi s u} d u
$$

denotes the $s$-th Fourier coefficient of $\mathcal{F}_{u ; \omega_{j_{1}}, \ldots, \omega_{j_{k-1}}}$ and is Hilbert-Schmidt. Furthermore,

$$
\left\|\operatorname{cum}\left(D_{\omega_{j_{1}}}^{(T)}, \ldots, D_{\omega_{j_{k}}}^{(T)}\right)\right\|_{2} \leqslant \frac{C}{T^{k / 2-1}\left|j_{1}+\cdots+j_{k}\right|^{l}}+O\left(\frac{1}{T^{k / 2}}\right)
$$

for some constant $C>0$.

In case the process does not depend on $u$, we have $\tilde{\mathcal{F}}_{s ; \omega_{j_{1}}, \ldots, \omega_{j_{k-1}}}=O_{H}$ for $s \neq 0$. That is, the operator $\tilde{\mathcal{F}}_{s ; \omega_{j_{1}}, \ldots, \omega_{j_{2 k-1}}}$ maps any $\phi \in L^{2}\left([0,1]^{k}, \mathbb{C}\right)$ to the origin for $s \neq 0$.

Proof. The first line of (4.28) follows from replacing $\operatorname{cum}\left(X_{t_{1}}^{(T)}, \ldots, X_{t_{k-1}}^{(T)}, X_{t_{k}}^{(T)}\right)$ with $c_{t_{k} / T ; t_{1}-t_{k}, \ldots, t_{k-1}-t_{k}}$ and Lemma B.1.3. The second line follows because the discretization of the integral is an operation of order $O\left(T^{-2}\right)$.

By Assumption 4.4.6 (iv), the kernel of $\frac{\partial}{\partial u} \mathcal{F}_{u ; \omega_{1}, \ldots, \omega_{k-1}}$ satisfies

$$
\left\|\sup _{u} \frac{\partial}{\partial u} f_{u ; \omega_{1}, \ldots, \omega_{k-1}}\right\|_{2} \leqslant \frac{1}{(2 \pi)^{k-1}} \sum_{t_{1}, \ldots, t_{k}}\left\|\kappa_{k ; t_{1}-t_{k}, \ldots, t_{k-1}-t_{k}}\right\|_{2}<\infty .
$$

The Dominated Convergence Theorem therefore yields

$$
\sup _{u, \omega_{1}, \ldots, \omega_{k-1}}\left\|\frac{\partial}{\partial u} f_{u, \omega_{1}, \ldots, \omega_{k-1}}\right\|_{2}<\infty .
$$

Finally, integration by parts for a periodic function in $L^{2}\left([0,1]^{k}, \mathbb{C}\right)$ with existing $n$-th directional derivative in $u$, yields

$$
\begin{aligned}
& \left\|\tilde{f}_{s ; \omega_{j_{1}}, \ldots, \omega_{j_{k-1}}}\right\|_{2}^{2} \\
& =\int_{[0,1]^{k}}\left|\left[\frac{\frac{\partial^{n-1}}{\partial u^{n-1}} f_{u ; \omega_{j_{1}}, \ldots, \omega_{j_{k-1}}}(\boldsymbol{\tau})}{(-\mathrm{i} 2 \pi s)^{n-1}} e^{-\mathrm{i} s 2 \pi u}\right]_{0}^{1}-\int_{0}^{1} \frac{e^{-\mathrm{i} s 2 \pi u}}{(-\mathrm{i} 2 \pi s)^{n}} \frac{\partial^{n}}{\partial u^{n}} f_{u ; \omega_{j_{1}}, \ldots, \omega_{j_{k-1}}}(\boldsymbol{\tau}) d u\right|^{2} d \boldsymbol{\tau} \\
& =\int_{[0,1]^{k+2}} \frac{1}{(2 \pi s)^{2 n}} e^{\mathrm{i} 2 \pi s(u-v)} \frac{\partial^{2}}{\partial u^{2}} f_{u ; \omega_{j_{1}}, \ldots, \omega_{j_{k-1}}}(\boldsymbol{\tau}) \frac{\partial^{2}}{\partial v^{2}} f_{v ; \omega_{j_{1}}, \ldots, \omega_{j_{k-1}}}(\boldsymbol{\tau}) d \boldsymbol{\tau} d u d v \\
& \leqslant \frac{1}{(2 \pi s)^{2 n}} \int_{[0,1]^{k+2}}\left|\frac{\partial^{2}}{\partial u^{2}} f_{u ; \omega_{j_{1}}, \ldots, \omega_{j_{k-1}}}(\boldsymbol{\tau}) \frac{\partial^{2}}{\partial v^{2}} f_{v ; \omega_{j_{1}}, \ldots, \omega_{j_{k-1}}}(\boldsymbol{\tau})\right| d \boldsymbol{\tau} d u d v \\
& \leqslant \frac{1}{(2 \pi s)^{2 n}} \int_{[0,1]^{2}}\left\|\frac{\partial^{2}}{\partial u^{2}} f_{u ; \omega_{j_{1}}, \ldots, \omega_{j_{k-1}}}\right\|\left\|_{2}\right\| \frac{\partial^{2}}{\partial v^{2}} f_{v ; \omega_{j_{1}}, \ldots, \omega_{j_{k-1}}} \|_{2} d u d v \\
& \leqslant \frac{1}{(2 \pi s)^{2 n}}\left(\sup _{u}\left\|\frac{\partial^{2}}{\partial u^{2}} f_{u ; \omega_{j_{1}}, \ldots, \omega_{j_{k-1}}}\right\|_{2}\right)^{2}<\infty,
\end{aligned}
$$


where the Cauchy-Schwarz Inequality was applied in the second-to-last equality. The interchange of integrals is justified by Fubini's Theorem. Thus,

$$
\sup _{\omega_{1}, \ldots, \omega_{k-1}}\left\|\tilde{f}_{s ; \omega_{j_{1}}, \ldots, \omega_{j_{k-1}}}\right\|_{2} \leqslant \frac{1}{(2 \pi)^{2 n}} \sup _{u, \omega_{1}, \ldots, \omega_{n}}\left\|\frac{\partial^{n}}{\partial u^{n}} f_{u ; \omega_{j_{1}}, \ldots, \omega_{j_{k-1}}}\right\|_{2}|s|^{-n} .
$$

and (4.30) follows from Assumption 4.4.6 (iv).

Lemma B.1.6 (Cumulants of the fDFT under local stationarity). Let $\left(X_{t, T}: t \leqslant\right.$ $T, T \in \mathbb{N}$ ) be a $k$-th order locally stationary process in $H$ satisfying Assumption 4.4.6. The cumulant operator of the local fDFT satisfies

$$
\begin{aligned}
\operatorname{cum}\left(D_{\omega_{j_{1}}}^{(T)}, \ldots, D_{\omega_{j_{k}}}^{(T)}\right) & =\frac{(2 \pi)^{k / 2-1}}{T^{k / 2}} \sum_{t=0}^{T-1} \mathcal{F}_{\frac{t}{T} ; \omega_{j_{1}}, \ldots, \omega_{j_{k-1}}} e^{-\mathrm{i} \sum_{l=1}^{k} t \omega_{j_{l}}}+R_{T, k} \\
& =\frac{(2 \pi)^{k / 2-1}}{T^{k / 2-1}} \tilde{\mathcal{F}}_{j_{1}+\ldots+j_{k} ; \omega_{j_{1}}, \ldots, \omega_{j_{k-1}}}+R_{T, k}
\end{aligned}
$$

where the remainder satisfies $\left\|R_{T, k}\right\|_{2}=O\left(T^{-k / 2}\right)$.

This lemma is a slight reformulation of the first part of Lemma B.1.5 and provides the locally stationary version of Lemma B.1.2. Both (4.25) and (4.33) belong to the class of Hilbert-Schmidt operators for $k \geqslant 2$. In particular, the operators (4.25) and (4.33) belong to $S_{1}(H)$ for $k=2$ if the process is driven by a functional white noise process, which can be shown using Parseval's Identity (Panaretos and Tavakoli, 2013b; van Delft and Eichler, 2016, respectively). The corollary below is a direct consequence of Lemma B.1.5.

Corollary B.1.1. If Assumption 4.4.6 holds with $\ell=2$, then

(i) $\left\|\operatorname{cum}\left(D_{\omega_{j_{1}}}^{(T)}, \ldots, D_{\omega_{j_{k}}}^{(T)}\right)\right\|_{2} \leqslant \frac{C}{T^{k / 2-1}\left|j_{1}+\cdots+j_{k}\right|^{2}}+O\left(\frac{1}{T^{k / 2}}\right)$;

(ii) $\sup _{\omega} \sum_{s \in \mathbb{Z}}\left\|\tilde{\mathcal{F}}_{s ; \omega}\right\|_{2} \leqslant \infty$.

Proof. For completeness, we elaborate on part (ii) of Corollary B.1.2. Note that

$$
\left\|\tilde{\mathcal{F}}_{0: \omega}\right\|_{2} \leqslant \sup _{\omega, u}\left\|\mathcal{F}_{u, \omega}\right\|_{2}<\sum_{h}\left\|\kappa_{2, h}\right\|_{2}<\infty .
$$

The $p$-harmonic series for $p=2$ then yields

$$
\sup _{\omega} \sum_{s \in \mathbb{Z}}\left\|\tilde{\mathcal{F}}_{s ; \omega}\right\|\left\|_{2} \leqslant \sum_{h}\right\| \kappa_{2, h} \|_{2}\left(1+\frac{1}{(2 \pi)^{4}} \frac{\pi^{2}}{3}\right)<\infty
$$

where the constant $(2 \pi)^{-4}$ follows from (4.32). 


\section{B.2 Error bound for the denominator of the test statistic}

A bound needs to be obtained on the error resulting from the replacement of the unknown spectral operators with consistent estimators. It will be sufficient to consider a bound on

$$
\sqrt{T}\left|\gamma_{h}^{(T)}\left(l_{1}, l_{2}\right)-\hat{\gamma}_{h}^{(T)}\left(l_{1}, l_{2}\right)\right|
$$

for all $l_{1}, l_{2} \in \mathbb{N}$, where $\gamma^{(T)}$ is defined in (4.11). Consider the function $g(x)=x^{-1 / 2}$, $x>0$ and notice that

$$
\gamma_{h}^{(T)}\left(l_{1}, l_{2}\right)-\hat{\gamma}_{h}^{(T)}\left(l_{1}, l_{2}\right)=\frac{1}{T} \sum_{j=1}^{T} D_{\omega_{j}}^{\left(l_{1}\right)} D_{-\omega_{j+h}}^{\left(l_{2}\right)}\left[g\left(\mathcal{F}_{\omega_{j}}^{\left(l_{1}, l_{1}\right)} \mathcal{F}_{\omega_{j+h}}^{\left(l_{2}, l_{2}\right)}\right)-g\left(\hat{\mathcal{F}}_{\omega_{j}}^{\left(l_{1}, l_{1}\right)} \hat{\mathcal{F}}_{\omega_{j+h}}^{\left.l_{2}, l_{2}\right)}\right)\right] .
$$

Given the assumption $\inf _{\omega}\left\langle\mathcal{F}_{\omega}(\psi), \psi\right\rangle>0$ for all $\psi \in H$ is satisfied, continuity of the inner product implies that for fixed $l_{1}, l_{2}$ the mean value theorem may be applied to find

$$
\begin{aligned}
\gamma_{h}^{(T)}\left(l_{1}, l_{2}\right) & -\hat{\gamma}_{h}^{(T)}\left(l_{1}, l_{2}\right) \\
& =\frac{1}{T} \sum_{j=1}^{T} D_{\omega_{j}}^{\left(l_{1}\right)} D_{-\omega_{j+h}}^{\left(l_{2}\right)}\left[\left.\frac{\partial g(x)}{\partial x}\right|_{x=\breve{\mathcal{F}}_{\omega_{j}}^{\left(l_{1}, l_{1}\right)} \breve{\mathcal{F}}_{\omega_{j+h}}^{\left(l_{2}, l_{2}\right)}}\left(\hat{\mathcal{F}}_{\omega_{j}}^{\left(l_{1}, l_{1}\right)} \hat{\mathcal{F}}_{\omega_{j+h}}^{\left(l_{2}, l_{2}\right)}-\mathcal{F}_{\omega_{j}}^{\left(l_{1}, l_{1}\right)} \mathcal{F}_{\omega_{j+h}}^{\left(l_{2}, l_{2}\right)}\right)\right],
\end{aligned}
$$

where $\breve{\mathcal{F}}_{\omega_{j}}^{\left(l_{1}, l_{1}\right)} \breve{\mathcal{F}}_{\omega_{j+h}}^{\left(l_{2}, l_{2}\right)}$ lies in between $\hat{\mathcal{F}}_{\omega_{j}}^{\left(l_{1}, l_{1}\right)} \hat{\mathcal{F}}_{\omega_{j+h}}^{\left(l_{2}, l_{2}\right)}$ and $\mathcal{F}_{\omega_{j}}^{\left(l_{1}, l_{1}\right)} \mathcal{F}_{\omega_{j+h}}^{\left(l_{2}, l_{2}\right)}$. Because of uniform convergence of $\hat{\mathcal{F}}_{\omega_{j}} \in S_{2}(H)$ with respect to $\omega$, it follows that

$$
\begin{aligned}
& \sqrt{T}\left|\gamma_{h}^{(T)}\left(l_{1}, l_{2}\right)-\hat{\gamma}_{h}^{(T)}\left(l_{1}, l_{2}\right)\right|= \\
& O_{p}(1)\left|\frac{1}{T} \sum_{j=1}^{T} \frac{D_{\omega_{j}}^{\left(l_{1}\right)} D_{-\omega_{j+h}}^{\left(l_{2}\right)}}{\left(\mathcal{F}_{\omega_{j}}^{\left(l_{1}, l_{1}\right)} \mathcal{F}_{\omega_{j+h}}^{\left(l_{2}, l_{2}\right)}\right)^{3 / 2}}\left(\hat{\mathcal{F}}_{\omega_{j}}^{\left(l_{1}, l_{1}\right)} \hat{\mathcal{F}}_{\omega_{j+h}}^{\left(l_{2}, l_{2}\right)}-\mathcal{F}_{\omega_{j}}^{\left(l_{1}, l_{1}\right)} \mathcal{F}_{\omega_{j+h}}^{\left(l_{2}, l_{2}\right)}\right)\right| .
\end{aligned}
$$

Proof of Theorem 4.4.2 and Theorem 4.4.8. In order to prove both theorems, partition (4.35) as follows

$$
\begin{aligned}
& J_{1}\left(l, l_{2}\right)=\frac{1}{\sqrt{T}} \sum_{j=1}^{T} \frac{D_{\omega_{j}}^{\left(l_{1}\right)} D_{-\omega_{j+h}}^{\left(l_{2}\right)}-\mathbb{E}\left[D_{\omega_{j}}^{\left(l_{1}\right)} D_{-\omega_{j+h}}^{\left(l_{2}\right)}\right]}{\left(\mathcal{F}_{\omega_{j}}^{\left(l_{1}, l_{1}\right)} \mathcal{F}_{\omega_{j+h}}^{\left(l_{2}, l_{2}\right)}\right)^{3 / 2}} \hat{\mathcal{F}}_{\omega_{j}}^{\left(l_{1}, l_{1}\right)}\left(\hat{\mathcal{F}}_{\omega_{j+h}}^{\left(l_{2}, l_{2}\right)}-\mathcal{F}_{\omega_{j+h}}^{\left(l_{2}, l_{2}\right)}\right), \\
& J_{2}\left(l, l_{2}\right)=\frac{1}{\sqrt{T}} \sum_{j=1}^{T} \frac{D_{\omega_{j}}^{\left(l_{1}\right)} D_{-\omega_{j+h}}^{\left(l_{2}\right)}-\mathbb{E}\left[D_{\omega_{j}}^{\left(l_{1}\right)} D_{-\omega_{j+h}}^{\left(l_{2}\right)}\right]}{\left(\mathcal{F}_{\omega_{j}}^{\left(l_{1}, l_{1}\right)} \mathcal{F}_{\omega_{j+h}}^{\left(l_{2}, l_{2}\right)}\right)^{3 / 2}} \mathcal{F}_{\omega_{j+h}}^{\left(l_{2}, l_{2}\right)}\left(\hat{\mathcal{F}}_{\omega_{j}}^{\left(l_{1}, l_{1}\right)}-\mathcal{F}_{\omega_{j}}^{\left(l_{1}, l_{1}\right)}\right), \\
& J_{3}\left(l, l_{2}\right)=\frac{1}{\sqrt{T}} \sum_{j=1}^{T} \frac{\mathbb{E}\left[D_{\omega_{j}}^{\left(l_{1}\right)} D_{-\omega_{j+h}}^{\left(l_{2}\right)}\right]}{\left(\mathcal{F}_{\omega_{j}}^{\left(l_{1}, l_{1}\right)} \mathcal{F}_{\omega_{j+h}}^{\left(l_{2}, l_{2}\right)}\right)^{3 / 2}}\left(\hat{\mathcal{F}}_{\omega_{j}}^{\left(l_{1}, l_{1}\right)} \hat{\mathcal{F}}_{\omega_{j+h}}^{\left(l_{2}, l_{2}\right)}-\mathbb{E}\left[\hat{\mathcal{F}}_{\omega_{j}}^{\left(l_{1}, l_{1}\right)} \hat{\mathcal{F}}_{\omega_{j+h}}^{\left(l_{2}, l_{2}\right)}\right]\right), \\
& J_{4}\left(l, l_{2}\right)=\frac{1}{\sqrt{T}} \sum_{j=1}^{T} \frac{\mathbb{E}\left[D_{\omega_{j}}^{\left(l_{1}\right)} D_{-\omega_{j+h}}^{\left(l_{2}\right)}\right]}{\left(\mathcal{F}_{\omega_{j}}^{\left(l_{1}, l_{1}\right)} \mathcal{F}_{\omega_{j+h}}^{\left(l_{2}, l_{2}\right)}\right)^{3 / 2}}\left(\mathbb{E}\left[\hat{\mathcal{F}}_{\omega_{j}}^{\left(l_{1}, l_{1}\right)} \hat{\mathcal{F}}_{\omega_{j+h}}^{\left(l_{2}, l_{2}\right)}\right]-\mathcal{F}_{\omega_{j}}^{\left(l_{1}, l_{1}\right)} \mathcal{F}_{\omega_{j+h}}^{\left(l_{2}, l_{2}\right)}\right) .
\end{aligned}
$$


The proof of both theorems are based on Corollary B.2.1 and Lemmas B.2.1-B.2.4 below. These results together with an application of the Cauchy-Schwarz Inequality yield

(i) $\left|J_{1}\right|=O_{p}\left(\frac{1}{\sqrt{b T}}+b^{2}\right)$,

(ii) $\left|J_{2}\right|=\left\{\begin{array}{lr}O_{p}\left(\frac{1}{\sqrt{b} T}+\frac{b^{2}}{\sqrt{T}}\right) & \text { under Assumption 4.4.1, } \\ O_{p}\left(\frac{1}{\sqrt{b T}}+b^{2}\right) & \text { under Assumption 4.4.6, }\end{array}\right.$

(iii) $\left|J_{3}\right|= \begin{cases}O_{p}\left(\frac{1}{\sqrt{b} T}\right) & \text { under Assumption 4.4.1, } \\ O_{p}\left(\frac{1}{\sqrt{b T}}\right) & \text { under Assumption 4.4.6, }\end{cases}$

(iv) $\left|J_{4}\right|= \begin{cases}O\left(b^{2}+\frac{1}{b T}\right) & \text { under Assumption 4.4.1. } \\ O\left(\sqrt{T} b^{2}+\frac{1}{b \sqrt{T}}\right) & \text { under Assumption 4.4.6. }\end{cases}$

Minkowski's Inequality then gives the result.

Corollary B.2.1. Under Assumption 4.4.1,

$$
\mathbb{E}\left[\left\|\hat{\mathcal{F}}_{\omega}^{(T)}-\mathcal{F}_{\omega}\right\|_{2}^{2}\right]=O\left(\frac{1}{b T}+b^{4}\right) \quad(T \rightarrow \infty),
$$

while, under Assumption 4.4.6,

$$
\mathbb{E}\left[\left\|\hat{\mathcal{F}}_{\omega}^{(T)}-G_{\omega}\right\|_{2}^{2}\right]=O\left(\frac{1}{b T}+b^{4}\right) \quad(T \rightarrow \infty) .
$$

Proof. See Panaretos and Tavakoli (2013b, Theorem 3.6) and Theorem 4.4.7, respectively.

Lemma B.2.1. Let $\left(g_{j}^{\left(l_{1}, l_{2}\right)}: j \in \mathbb{Z}\right)$ be a bounded sequence in $\mathbb{C}$ for all $l_{1}, l_{2} \in \mathbb{N}$ such that $\inf _{j} g_{j}^{\left(l_{1}, l_{2}\right)}>0$. Under Assumption 4.4.1 and under Assumption 4.4.6,

$$
\mathbb{E}\left[\left|\frac{1}{\sqrt{T}} \sum_{j=1}^{T} g_{j}^{\left(l_{1}, l_{2}\right)}\left(D_{\omega_{j}}^{\left(l_{1}\right)} D_{-\omega_{j+h}}^{\left(l_{2}\right)}-\mathbb{E}\left[D_{\omega_{j}}^{\left(l_{1}\right)} D_{-\omega_{j+h}}^{\left(l_{2}\right)}\right]\right) \hat{\mathcal{F}}_{\omega_{j}}^{\left(l_{1}, l_{1}\right)}\right|^{2}\right]=O(1) .
$$


Proof. Observe that

$$
\begin{aligned}
& \mathbb{E}\left[\left|\frac{1}{\sqrt{T}} \sum_{j=1}^{T} g_{j}^{\left(l_{1}, l_{2}\right)}\left(D_{\omega_{j}}^{\left(l_{1}\right)} D_{-\omega_{j+h}}^{\left(l_{2}\right)}-\mathbb{E}\left[D_{\omega_{j}}^{\left(l_{1}\right)} D_{-\omega_{j+h}}^{\left(l_{2}\right)}\right]\right) \hat{\mathcal{F}}_{\omega_{j}}^{\left(l_{1}, l_{1}\right)}\right|^{2}\right] \\
& =\mathbb{E}\left[\left|\frac{1}{\sqrt{T}} \sum_{j=1}^{T} g_{j}^{\left(l_{1}, l_{2}\right)}\left(D_{\omega_{j}}^{\left(l_{1}\right)} D_{-\omega_{j+h}}^{\left(l_{2}\right)}-E\left[D_{\omega_{j}}^{\left(l_{1}\right)} D_{-\omega_{j+h}}^{\left(l_{2}\right)}\right]\right) \frac{2 \pi}{b T} \sum_{j^{\prime}=1}^{T} K\left(\frac{\omega_{j^{\prime}}}{b}\right) D_{\omega_{j-j^{\prime}}}^{\left(l_{1}\right)} D_{\omega_{j^{\prime}-j}}^{\left(l_{1}\right)}\right|^{2}\right] \\
& =\frac{1}{T}\left(\frac{2 \pi}{b T}\right)^{2} \sum_{j_{1}, j_{2}=1}^{T} g_{j_{1}, j_{2}}^{\left(l_{1}, l_{2}\right)} \sum_{j_{1}^{\prime}, j_{2}^{\prime}=1}^{T} K\left(\frac{\omega_{j_{1}^{\prime}}}{b}\right) K\left(\frac{\omega_{j_{2}^{\prime}}}{b}\right) \mathbb{E}\left[\left\{\left(D_{\omega_{j_{1}}}^{\left(l_{1}\right)} D_{-\omega_{j_{1}+h}}^{\left(l_{2}\right)}-\mathbb{E}\left[D_{\omega_{j_{1}}}^{\left(l_{1}\right)} D_{-\omega_{j_{1}+h}}^{\left(l_{2}\right)}\right]\right)\right.\right. \\
& \left.\left.\times D_{\omega_{j_{1}-j_{1}^{\prime}}}^{\left(l_{1}\right)} D_{\omega_{j_{1}^{\prime}-j_{1}}^{\left(l_{1}\right)}}^{\left(l_{1}\right.}\right\}\left\{\left(D_{-\omega_{j_{2}}}^{\left(l_{1}\right)} D_{\omega_{j_{2}+h}}^{\left(l_{2}\right)}-\mathbb{E}\left[D_{-\omega_{j_{2}}}^{\left(l_{1}\right)} D_{\omega_{j_{2}+h}}^{\left(l_{2}\right)}\right]\right) D_{\omega_{j_{2}^{\prime}-j_{2}}}^{\left(l_{1}\right)} D_{\omega_{j_{2}-j_{2}^{\prime}}}^{\left(l_{1}\right)}\right\}\right] .
\end{aligned}
$$

Expanding the expectation in terms of cumulants, one obtains the structure

$$
\begin{aligned}
\mathbb{E}[(X- & \mathbb{E} X) Y][(W-E W) Z] \\
= & E[X Y W Z]-E[W] E[X Y Z]-E[Y W Z[E[X]+E[W] E[X] E[Y Z] \\
= & \operatorname{cum}(X, Y, W, Z)+\operatorname{cum}(X, Y, W) \operatorname{cum}(Z)+\operatorname{cum}(X, W, Z) \operatorname{cum}(Y) \\
& +\operatorname{cum}(X, Y) \operatorname{cum}(W, Z)+\operatorname{cum}(X, W) \operatorname{cum}(Y, Z)+\operatorname{cum}(X, Z) \operatorname{cum}(Y, W),
\end{aligned}
$$

where $X, Y, W, Z$ are products of random elements of $H$. Hence, by the product theorem for cumulants, only those products of cumulants have to be considered that lead to indecomposable partitions of the matrix below or of any sub-matrix (with the same column structure) with the exception that $(Y)$ or $(Z)$ is allowed to be decomposable but not within the same partition.

$$
\begin{aligned}
& (X) \quad D_{\omega_{j_{1}}}^{\left(l_{1}\right)} \quad D_{-\omega_{j_{1}+h}}^{\left(l_{2}\right)}
\end{aligned}
$$

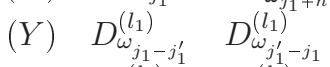

$$
\begin{aligned}
& (W) \quad D_{-\omega_{j_{2}}}^{\left(l_{1}\right)} \quad D_{\omega_{j_{2}+h}}^{\left(l_{2}\right)}
\end{aligned}
$$

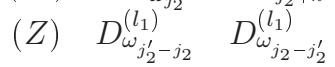

That is, the order of the error belonging to those partitions has to be investigated for which the cumulant terms that contain an element of the sets $\left\{D_{\omega_{j_{1}}}^{\left(l_{1}\right)}, D_{-\omega_{j_{1}+h}}^{\left(l_{2}\right)}\right\}$ and of $\left\{D_{-\omega_{j_{2}}}^{\left(l_{1}\right)} D_{\omega_{j_{2}+h}}^{\left(l_{2}\right)}\right\}$ also contain at least one element from another set. Because the process has zero-mean, it suffices consider partitions for which $m_{i} \geqslant 2$. By Lemma B.1.2, a cumulant of order $k$ upscaled by order $T$ will be of order $O\left(T^{-k / 2+1}\right)$ under $H_{0}$ and $O\left(T^{-k / 2+2}\right)$ under the alternative. This directly implies that only terms of the following form have to be investigated:

$$
\begin{aligned}
& \frac{1}{T}\left(\frac{2 \pi}{b T}\right)^{2} \sum_{j_{1}, j_{2}=1}^{T} \sum_{j_{1}^{\prime}, j_{2}^{\prime}=1}^{T} K\left(\frac{\omega_{j_{1}^{\prime}}}{b}\right) K\left(\frac{\omega_{j_{2}^{\prime}}}{b}\right) \text { cum }_{4} \operatorname{cum}_{2} \operatorname{cum}_{2}, \\
& \frac{1}{T}\left(\frac{2 \pi}{b T}\right)^{2} \sum_{j_{1}, j_{2}=1}^{T} \sum_{j_{1}^{\prime}, j_{2}^{\prime}=1}^{T} K\left(\frac{\omega_{j_{1}^{\prime}}}{b}\right) K\left(\frac{\omega_{j_{2}^{\prime}}}{b}\right) \operatorname{cum}_{3} \operatorname{cum}_{3} \operatorname{cum}_{2}, \\
& \frac{1}{T}\left(\frac{2 \pi}{b T}\right)^{2} \sum_{j_{1}, j_{2}=1}^{T} \sum_{j_{1}^{\prime}, j_{2}^{\prime}=1}^{T} K\left(\frac{\omega_{j_{1}^{\prime}}}{b}\right) K\left(\frac{\omega_{j_{2}^{\prime}}}{b}\right) \operatorname{cum}_{2} \operatorname{cum}_{2} \operatorname{cum}_{2} \operatorname{cum}_{2} .
\end{aligned}
$$


However, for a fixed partition $P=\left\{P_{1}, \ldots, P_{M}\right\}$,

$$
T \prod_{j=1}^{M} O\left(\frac{1}{T^{m_{j} / 2-1}}\right)
$$

from which it is also clear that (4.43) and (4.44) are at most going to be of order $O(1)$ under the alternative and of lower order under $H_{0}$. The term (4.45) could possibly of order $O(T)$. Further analysis can therefore be restricted to partitions of this form. As mentioned above, partitions in which a term contains an element of the sets $\left\{D_{\omega_{j_{1}}}^{\left(l_{1}\right)}, D_{-\omega_{j_{1}+h}}^{\left(l_{2}\right)}\right\}$ and of $\left\{D_{-\omega_{j_{2}}}^{\left(l_{1}\right)} D_{\omega_{j_{2}+h}}^{\left(l_{2}\right)}\right\}$ must contain at least one element from another set. It follows that partitions in which $m_{i}=2$ for all $i \in\{1, \ldots, M\}$, without any restrictions on the summations, are decomposable. We find the term of highest order is thus of the type

$$
\begin{aligned}
& \frac{1}{T} \sum_{j_{1}, j_{2}=1}^{T} g_{j_{1}, j_{2}}^{\left(l_{1}, l_{2}\right)}\left(\frac{2 \pi}{b T}\right)^{2} \sum_{j_{1}^{\prime}, j_{2}^{\prime}=1}^{T} K\left(\frac{\omega_{j_{1}^{\prime}}}{b}\right) K\left(\frac{\omega_{j_{2}^{\prime}}}{b}\right) \operatorname{cum}\left(D_{-\omega_{j_{1}+h}}^{\left(l_{2}\right)}, D_{\omega_{j_{2}+h}}^{\left(l_{2}\right)}\right) \operatorname{cum}\left(D_{-\omega_{j_{2}}}^{\left(l_{1}\right)}, D_{\omega_{j_{1}}}^{\left(l_{1}\right)}\right) \\
& \times \operatorname{cum}\left(D_{\omega_{j_{1}-j_{1}^{\prime}}}^{\left(l_{1}\right)} D_{\omega_{j_{1}-j_{1}^{\prime}}}^{\left(l_{1}\right)}\right) \operatorname{cum}\left(D_{\omega_{j_{2}^{\prime}-j_{2}}}^{\left(l_{1}\right)} D_{\omega_{j_{2}-j_{2}^{\prime}}}^{\left(l_{1}\right)}\right)=\sup _{j} h_{j}^{2\left(l_{1}, l_{2}\right)} O\left(T T^{-1}\right)=O(1),
\end{aligned}
$$

under the null of stationarity and under the alternative, where the error is uniform in $\omega$. The bound in case of the alternative follows from Corollary B.1.1. The result follows since by the positive definiteness of the spectral density operators $\sup _{j}\left|g_{j}^{\left(l_{1}, l_{2}\right)}\right|<\infty$ for all $l_{1}, l_{2} \in \mathbb{N}$.

Lemma B.2.2. Let $\left(g_{j}^{\left(l_{1}, l_{2}\right)}: j \in \mathbb{Z}\right)$ be a bounded sequence in $\mathbb{C}$ for all $l_{1}, l_{2} \in \mathbb{N}$ such that $\inf _{l_{1}, l_{2}} g_{j}^{\left(l_{1}, l_{2}\right)}>0$. Then,

$$
E\left[\left|\frac{1}{\sqrt{T}} \sum_{j=1}^{T} g_{j}^{\left(l_{1}, l_{2}\right)}\left(D_{\omega_{j}}^{\left(l_{1}\right)} D_{-\omega_{j+h}}^{\left(l_{2}\right)}-\mathbb{E}\left[D_{\omega_{j}}^{\left(l_{1}\right)} D_{-\omega_{j+h}}^{\left(l_{2}\right)}\right]\right)\right|^{2}\right]= \begin{cases}O\left(\frac{1}{T}\right) & \text { under } H_{0} . \\ O(1) & \text { under } H_{1} .\end{cases}
$$

Proof. Notice that

$$
\begin{aligned}
E & {\left[\left|\frac{1}{\sqrt{T}} \sum_{j=1}^{T} g_{j}^{\left(l_{1}, l_{2}\right)}\left(D_{\omega_{j}}^{\left(l_{1}\right)} D_{-\omega_{j+h}}^{\left(l_{2}\right)}-\mathbb{E}\left[D_{\omega_{j}}^{\left(l_{1}\right)} D_{-\omega_{j+h}}^{\left(l_{2}\right)}\right]\right)\right|^{2}\right] } \\
= & \frac{1}{T} \sum_{j_{1}, j_{2}=1}^{T}\left(g_{j_{1}, j_{2}}^{\left(l_{1}, l_{2}\right)} \mathbb{E}\left[D_{\omega_{j_{1}}}^{\left(l_{1}\right)} D_{-\omega_{j_{1}+h}}^{\left(l_{2}\right)} D_{-\omega_{j_{2}}}^{\left(l_{1}\right)} D_{\omega_{j_{2}+h}}^{\left(l_{2}\right)}\right]-\mathbb{E}\left[D_{\omega_{j_{1}}}^{\left(l_{1}\right)} D_{-\omega_{j_{1}+h}}^{\left(l_{2}\right)}\right] \mathbb{E}\left[D_{-\omega_{j_{2}}}^{\left(l_{1}\right)} D_{\omega_{j_{2}+h}}^{\left(l_{2}\right)}\right]\right) \\
= & \frac{1}{T} \sum_{j_{1}, j_{2}=1}^{T}\left(g_{j_{1}, j_{2}}^{\left(l_{1}, l_{2}\right)} \operatorname{cum}\left(D_{\omega_{j_{1}}}^{\left(l_{1}\right)}, D_{-\omega_{j_{1}+h}}^{\left(l_{2}\right)}, D_{-\omega_{j_{2}}}^{\left(l_{1}\right)} D_{\omega_{j_{2}+h}}^{\left(l_{2}\right)}\right)+\right. \\
& \left.\quad \operatorname{cum}\left(D_{\omega_{j_{1}}}^{\left(l_{1}\right)}, D_{-\omega_{j_{2}}}^{\left(l_{1}\right)}\right) \operatorname{cum}\left(D_{-\omega_{j_{1}+h}}^{\left(l_{2}\right)}, D_{\omega_{j_{2}+h}}^{\left(l_{2}\right)}\right)+\operatorname{cum}\left(D_{\omega_{j_{1}}}^{\left(l_{1}\right)}, D_{\omega_{j_{2}+h}}^{\left(l_{2}\right)}\right) \operatorname{cum}\left(D_{-\omega_{j_{1}+h}}^{\left(l_{2}\right)}, D_{-\omega_{j_{2}}}^{\left(l_{1}\right)}\right)\right) .
\end{aligned}
$$

Under the null, this is therefore of the order $O\left(T / T^{2}+1 / T\right)=O(1 / T)$, where is uniform over $\omega$. Under the alternative, by Corrolary B.1.1, the last term can be 
estimated by

$$
\begin{aligned}
& \frac{1}{T} \sum_{j_{1}, j_{2}=1}^{T} g_{j_{1}, j_{2}}^{\left(l_{1}, l_{2}\right)}\left(\frac{1}{T} G_{\omega_{j_{1}},-\omega_{j_{1}+h},-\omega_{j_{2}}}^{\left(l_{1} l_{2}, l_{1} l_{2}\right)}+\tilde{\mathcal{F}}_{j_{1}-j_{2} ; \omega_{j_{1}}}^{\left(l_{1}, l_{1}\right)} \tilde{\mathcal{F}}_{-j_{1}+j_{2} ;-\omega_{j_{1}+h}}^{\left(l_{2}, l_{2}\right)}\right. \\
& \left.\quad+\tilde{\mathcal{F}}_{j_{1}+j_{2}+h ; \omega_{j_{1}}}^{\left(l_{1}, l_{2}\right)} \tilde{\mathcal{F}}_{-j_{1}-j_{2}-h ;-\omega_{j_{1}+h}}^{\left(l_{2}, l_{1}\right)}+O\left(\frac{1}{T}\right)\right) \\
& \leqslant \sup _{j}\left|g_{j}^{\left(l_{1}, l_{2}\right)}\right|^{2} \frac{1}{T} \sum_{j_{1}, j_{2}=1}^{T}\left(\frac{1}{T} G_{\omega_{j_{1}},-\omega_{j_{1}+h},-\omega_{j_{2}}}^{\left(l_{1} l_{2}, l_{1} l_{2}\right)}+\tilde{\mathcal{F}}_{j_{1}-j_{2} ; \omega_{j_{1}}}^{\left(l_{1}, l_{1}\right)} \tilde{\mathcal{F}}_{-j_{1}+j_{2} ;-\omega_{j_{1}+h}}^{\left(l_{2}, l_{2}\right)}\right. \\
& \left.\quad+\tilde{\mathcal{F}}_{j_{1}+j_{2}+h ; \omega_{j_{1}}}^{\left(l_{1}, l_{2}\right)} \tilde{\mathcal{F}}_{-j_{1}-j_{2}-h ;-\omega_{j_{1}+h}}^{\left(l_{2}, l_{1}\right)}+O\left(\frac{1}{T}\right)\right) \\
& \leqslant \sup _{j}\left|g_{j}^{\left(l_{1}, l_{2}\right)}\right|^{2}\left(\sum_{t_{1}, t_{2}, t_{3}}\left\|\kappa_{4 ; t_{1}, t_{2}, t_{3}}\right\|_{2}\left\|\psi_{l_{1}}\right\|_{2}^{2} \mid \psi_{l_{2}}\left\|_{2}^{2}+C \sum_{t}\right\| \kappa_{2 ; t} \|_{2}\right)=O(1),
\end{aligned}
$$

for some constant C.

Lemma B.2.3. Let $\left(g_{j}^{\left(l_{1}, l_{2}\right)}: j \in \mathbb{Z}\right)$ be a bounded sequence in $\mathbb{C}$ for all $l_{1}, l_{2} \in \mathbb{N}$ such that $\inf _{l_{1}, l_{2}} g_{j}^{\left(l_{1}, l_{2}\right)}>0$. Then,

$$
\begin{gathered}
\left.\mathbb{E}\left[\mid \frac{1}{\sqrt{T}} \sum_{j=1}^{T} g_{j}^{\left(l_{1}, l_{2}\right)} \mathbb{E}\left[D_{\omega_{j}}^{\left(l_{1}\right)} D_{-\omega_{j+h}}^{\left(l_{2}\right)}\right]\left(\hat{\mathcal{F}}_{\omega_{j}}^{\left(l_{1}, l_{1}\right)} \hat{\mathcal{F}}_{\omega_{j+h}}^{\left(l_{2}, l_{2}\right)}-\mathbb{E}\left[\hat{\mathcal{F}}_{\omega_{j}}^{\left(l_{1}, l_{1}\right)} \hat{\mathcal{F}}_{\omega_{j+h}}^{\left(l_{2}, l_{2}\right)}\right]\right)\right]\right] \\
= \begin{cases}O\left(\frac{1}{\sqrt{b} T}\right) & \text { under Assumption 4.4.1. } \\
O\left(\frac{1}{\sqrt{b T}}\right) & \text { under Assumption 4.4.6. }\end{cases}
\end{gathered}
$$

Proof. Observe first that by the Cauchy-Schwarz Inequality,

$$
\begin{aligned}
\mathbb{E}\left[\left|J_{3}\left(l_{1}, l_{2}\right)\right|\right] & \leqslant \sup _{j}\left|g_{j}^{\left(l_{1}, l_{2}\right)} \mathbb{E}\left[D_{\omega_{j}}^{\left(l_{1}\right)} D_{-\omega_{j+h}}^{\left(l_{2}\right)}\right]\right| \\
& \times\left(\mathbb{E}\left[\left|\frac{1}{\sqrt{T}} \sum_{j=1}^{T}\left(\hat{\mathcal{F}}_{\omega_{j}}^{\left(l_{1}, l_{1}\right)} \hat{\mathcal{F}}_{\omega_{j+h}}^{\left(l_{2}, l_{2}\right)}-\mathbb{E}\left[\hat{\mathcal{F}}_{\omega_{j}}^{\left(l_{1}, l_{1}\right)} \hat{\mathcal{F}}_{\omega_{j+h}}^{\left(l_{2}, l_{2}\right)}\right]\right)\right|^{2}\right]\right)^{1 / 2},
\end{aligned}
$$

which follows because the term over which the supremum is taken is deterministic. In particular, it is of order $O\left(T^{-1}\right)$ under the null and $O\left(h^{-2}\right)$ under the alternative. To find a bound on

$$
\mathbb{E}\left[\left|\frac{1}{\sqrt{T}} \sum_{j=1}^{T}\left(\hat{\mathcal{F}}_{\omega_{j}}^{\left(l_{1}, l_{1}\right)} \hat{\mathcal{F}}_{\omega_{j+h}}^{\left(l_{2}, l_{2}\right)}-\mathbb{E}\left[\hat{\mathcal{F}}_{\omega_{j}}^{\left(l_{1}, l_{1}\right)} \hat{\mathcal{F}}_{\omega_{j+h}}^{\left(l_{2}, l_{2}\right)}\right]\right)\right|^{2}\right]
$$


we proceed similarly as in the proof of Lemma B.2.1. Observe that,

$$
\begin{aligned}
& \mathbb{E}\left[\left|\frac{1}{\sqrt{T}} \sum_{j=1}^{T}\left(\hat{\mathcal{F}}_{\omega_{j}}^{\left(l_{1}, l_{1}\right)} \hat{\mathcal{F}}_{\omega_{j+h}}^{\left(l_{2}, l_{2}\right)}-\mathbb{E} \hat{\mathcal{F}}_{\omega_{j}}^{\left(l_{1}, l_{1}\right)} \hat{\mathcal{F}}_{\omega_{j+h}}^{\left(l_{2}, l_{2}\right)}\right)\right|^{2}\right] \\
& =\frac{1}{T}\left(\frac{2 \pi}{b T}\right)^{2} \sum_{j_{1}, j_{2}=1}^{T} \sum_{j_{1}^{\prime}, j_{2}^{\prime}, j_{3}^{\prime}, j_{4}^{\prime}=1}^{T} \prod_{i=1}^{4} K\left(\frac{\omega_{j_{i}^{\prime}}}{b}\right)
\end{aligned}
$$

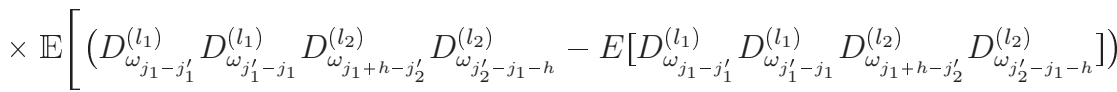

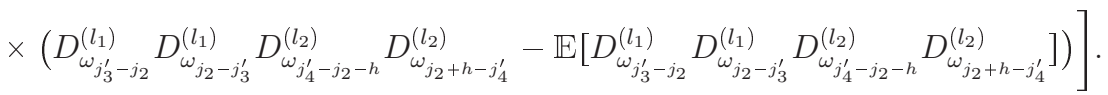

Write

$$
\mathbb{E}[(X-\mathbb{E} X)][(Y-E Y)]=\operatorname{cum}(X, Y)-\operatorname{cum}(X) \operatorname{cum}(Y)
$$

for products $X, W$ of random elements of $H$. When expanding this in terms of cumulants, we only have to consider those products of cumulants that lead to indecomposable partitions of the rows of the matrix below

$$
\begin{aligned}
& (X) \quad D_{\omega_{j_{1}-j_{1}^{\prime}}^{\prime}}^{\left(l_{1}\right)} D_{\omega_{j_{1}^{\prime}-j_{1}}}^{\left(l_{1}\right)} \quad D_{\omega_{j_{1}+h-j_{2}^{\prime}}}^{\left(l_{2}\right)} \quad D_{\omega_{j_{2}^{\prime}-j_{1}-h}^{\left(l_{2}\right)}}^{\left(l_{1}\right)}
\end{aligned}
$$

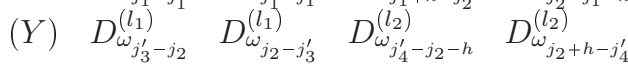

In order to satisfy this, in every partition there must be at least one term that contains both an element of $X$ and of $Y$. A similar reasoning as in the proof of B.2.1 indicates we will only have to consider partitions where $2 \leqslant m_{i} \leqslant 4$ for $i=1, \ldots, M$. In case of stationarity we only have to consider those with $m_{i}=2$ for all $i=1, \ldots, M$. In both cases at least one restriction in terms of the summation must occur in order for the partition to be decomposable. In particular, it can be verified that the partition of highest order is of the form

$$
\begin{aligned}
& \frac{1}{T} \sum_{j_{1}, j_{2}=1}^{T}\left(\frac{2 \pi}{b T}\right)^{4} \sum_{j_{1}^{\prime}, j_{2}^{\prime}, j_{3}^{\prime}, j_{4}^{\prime}=1}^{T} \prod_{i=1}^{4} K\left(\frac{\omega_{j_{i}^{\prime}}}{b}\right) \operatorname{cum}\left(D_{\omega_{j_{4}^{\prime}-j_{2}-h}}^{\left(l_{2}\right)} D_{\omega_{j_{2}+h-j_{4}^{\prime}}}^{\left(l_{2}\right)}\right) \operatorname{cum}\left(D_{\omega_{j_{1}+h-j_{2}^{\prime}}}^{\left(l_{2}\right)} D_{\omega_{j_{2}^{\prime}-j_{1}-h}}^{\left(l_{2}\right)}\right) \\
& \times \operatorname{cum}\left(D_{\omega_{j_{1}-j_{1}^{\prime}}}^{\left(l_{1}\right)}, D_{\omega_{j_{3}^{\prime}-j_{2}}}^{\left(l_{1}\right)}\right) \operatorname{cum}\left(D_{\omega_{j_{1}^{\prime}-j_{1}}}^{\left(l_{1}\right)}, D_{\omega_{j_{2}-j_{3}^{\prime}}}^{\left(l_{1}\right)}\right)
\end{aligned}
$$

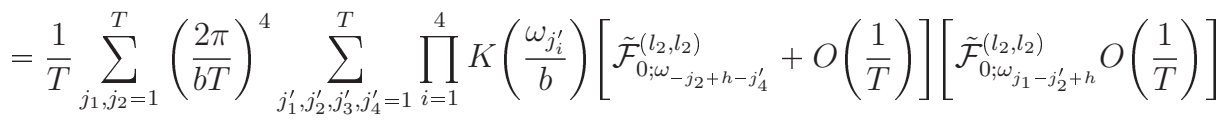

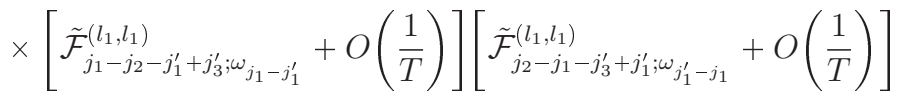

$$
\begin{aligned}
& \leqslant C \frac{1}{b T} \sup _{\omega}\left|G_{\omega}^{2\left(l_{2}, l_{2}\right)}\right| \frac{1}{T} \sum_{j_{1}, j_{2}=1}^{T}\left[\tilde{\mathcal{F}}_{j_{1}-j_{2} ; \omega_{j_{1}-j_{1}^{\prime}}}^{\left(l_{1}, l_{1}\right)}+O\left(\frac{1}{T}\right)\right]\left[\tilde{\mathcal{F}}_{j_{2}-j_{1} ; \omega_{j_{1}^{\prime}-j_{1}}}^{\left(l_{1}, l_{1}\right)}+O\left(\frac{1}{T}\right)\right]=O\left(\frac{1}{b T}\right)
\end{aligned}
$$

since $\left\|\tilde{\mathcal{F}}_{j_{1}-j_{2}-j_{1}^{\prime}+j_{3}^{\prime} ; \omega_{j_{1}-j_{1}^{\prime}}}\right\|_{2} \leqslant C\left|j_{1}-j_{2}-j_{1}^{\prime}+j_{3}^{\prime}\right|^{-2}$ and $\|K(x / b)\|_{\infty}=O(1)$, where the bandwidth leads to only $b T$ nonzero terms in the summation over $j_{1}$. The same 
bound can be shown to hold under stationarity. As before, the error is uniform with respect to $\omega$ which follows again from Corollary B.1.1.

Lemma B.2.4. Let $\left(g_{j}^{\left(l_{1}, l_{2}\right)}: j \in \mathbb{Z}\right)$ be a bounded sequence in $\mathbb{C}$ for all $l_{1}, l_{2} \in \mathbb{N}$ such that $\inf _{l_{1}, l_{2}} g_{j}^{\left(l_{1}, l_{2}\right)}>0$. Then,

$$
\begin{aligned}
& \left|\frac{1}{\sqrt{T}} \sum_{j=1}^{T} g_{j}^{\left(l_{1}, l_{2}\right)} \mathbb{E}\left[D_{\omega_{j}}^{\left(l_{1}\right)} D_{-\omega_{j+h}}^{\left(l_{2}\right)}\right]\left(\mathbb{E}\left[\hat{\mathcal{F}}_{\omega_{j}}^{\left(l_{1}, l_{1}\right)} \hat{\mathcal{F}}_{\omega_{j+h}}^{\left(l_{2}, l_{2}\right)}\right]-\mathcal{F}_{\omega_{j}}^{\left(l_{1}, l_{1}\right)} \mathcal{F}_{\omega_{j+h}}^{\left(l_{2}, l_{2}\right)}\right)\right| \\
& = \begin{cases}O\left(b^{2}+\frac{1}{b T}\right) & \text { under Assumption 4.4.1. } \\
O\left(\sqrt{T} b^{2}+\frac{1}{b \sqrt{T}}\right) & \text { under Assumption 4.4.6. }\end{cases}
\end{aligned}
$$

Proof. First note that

$$
\left|\frac{1}{\sqrt{T}} \sum_{j=1}^{T} g_{j}^{\left(l_{1}, l_{2}\right)} \mathbb{E}\left[D_{\omega_{j}}^{\left(l_{1}\right)} D_{-\omega_{j+h}}^{\left(l_{2}\right)}\right]\right|= \begin{cases}O\left(\frac{1}{\sqrt{T}}\right) & \text { under Assumption 4.4.1. } \\ O(\sqrt{T}) & \text { under Assumption 4.4.6. }\end{cases}
$$

Observe next that

$$
\begin{aligned}
\mathbb{E} & {\left[\hat{\mathcal{F}}_{\omega_{j}}^{\left(l_{1}, l_{1}\right)} \hat{\mathcal{F}}_{\omega_{j+h}}^{\left(l_{2}, l_{2}\right)}\right]-\mathcal{F}_{\omega_{j}}^{\left(l_{1}, l_{1}\right)} \mathcal{F}_{\omega_{j+h}}^{\left(l_{2}, l_{2}\right)} } \\
& =\left(\frac{2 \pi}{b T}\right)^{2} \sum_{j_{1}, j_{2}=1}^{T} K\left(\frac{\omega_{j_{1}}}{b}\right) K\left(\frac{\omega_{j_{2}}}{b}\right) \mathbb{E}\left[D_{\omega_{j-j_{1}}}^{\left(l_{1}\right)} D_{\omega_{j_{1}-j}}^{\left(l_{1}\right)} D_{\omega_{j+h-j_{2}}}^{\left(l_{2}\right)} D_{\omega_{j_{2}-j-h}}^{\left(l_{2}\right)}\right]-\mathcal{F}_{\omega_{j}}^{\left(l_{1}, l_{1}\right)} \mathcal{F}_{\omega_{j+h}}^{\left(l_{2}, l_{2}\right)} \\
& =\left(\frac{2 \pi}{b T}\right)^{2} \sum_{j_{1}, j_{2}=1}^{T} K\left(\frac{\omega_{j_{1}}}{b}\right) K\left(\frac{\omega_{j_{2}}}{b}\right)\left(\operatorname{cum}\left(D_{\omega_{j-j_{1}}}^{\left(l_{1}\right)}, D_{\omega_{j+h-j_{2}}}^{\left(l_{2}\right)}\right) \operatorname{cum}\left(D_{\omega_{j_{1}-j}}^{\left(l_{1}\right)}, D_{\omega_{j_{2}-j-h}}^{\left(l_{2}\right)}\right)\right. \\
& \left.+\operatorname{cum}\left(D_{\omega_{j-j_{1}}}^{\left(l_{1}\right)}, D_{\omega_{j_{2}-j-h}}^{\left(l_{2}\right)}\right) \operatorname{cum}\left(D_{\omega_{j_{1}-j}}^{\left(l_{1}\right)}, D_{\omega_{j+h-j_{2}}}^{\left(l_{2}\right)}\right)\right)+O\left(b^{2}+\frac{1}{b T}\right) .
\end{aligned}
$$

Here, it was used that $\mathbb{E}\left[\left|\hat{\mathcal{F}}_{\omega_{j}}^{\left(l_{1}, l_{1}\right)}-\mathcal{F}_{\omega_{j}}^{\left(l_{1}, l_{1}\right)}\right|\right] \leqslant \mathbb{E}\left[\left\|\hat{\mathcal{F}}_{\omega_{j}}-\mathcal{F}_{\omega_{j}} \mid\right\|_{2}\right]\left\|\psi_{l_{1}}\right\|_{2}\left\|\psi_{l_{1}}\right\|_{2}=O\left(b^{2}+\right.$ $1 / b T$ ) under $H_{0}$. The same bound holds under the alternative, where $\mathcal{F}_{\omega}$ is replaced with the integrated spectrum $G_{\omega}$. Under the alternative, write

$$
\begin{aligned}
\left(\frac{2 \pi}{b T}\right)^{2} & \sum_{j_{1}, j_{2}=1}^{T} K\left(\frac{\omega_{j_{1}}}{b}\right) K\left(\frac{\omega_{j_{2}}}{b}\right)\left(\tilde{\mathcal{F}}_{j_{2}-j_{1}-h ; \omega_{j-j_{1}}}^{\left(l_{1}, l_{2}\right)} \tilde{\mathcal{F}}_{j_{1}+h-j_{2} ; \omega_{j_{1}-j}}^{\left(l_{1}, l_{2}\right)}+\tilde{\mathcal{F}}_{h-j_{1}-j_{2} ; \omega_{j-j_{1}}}^{\left(l_{1}, l_{2}\right)} \tilde{\mathcal{F}}_{j_{1}+j_{2}-h ; \omega_{j_{1}-j}}^{\left(l_{1}, l_{2}\right)}\right. \\
+ & O\left(\frac{1}{T}\right)+O\left(b^{2}+\frac{1}{b T}\right)=O\left(b^{2}+\frac{1}{b T}\right)
\end{aligned}
$$

where Corollary B.1.1 was applied and where we used that the bandwidth leads to only $b T$ nonzero terms in the summation. Under $H_{0}$, a similar argument shows that the term is of order $O\left(b^{2}+1 / b T\right)$. The result now follows. 


\section{B.3 Convergence of finite-dimensional distribu- tions}

Theorem B.3.1. Let Lemma B.1.2 be satisfied for some finite $k \geqslant 3$. Then, for all $l_{i}, l_{i}{ }^{\prime} \in \mathbb{N}$ and $h_{i} \in \mathbb{Z}$ with $i=1, \ldots, k$,

$$
\frac{1}{T^{k / 2}} \operatorname{cum}\left(w_{h_{1}}^{(T)}\left(\psi_{l_{1} l_{1}{ }^{\prime}}\right), \ldots, w_{h_{k}}^{(T)}\left(\psi_{l_{k} l_{k^{\prime}}}\right)\right)=o(1) \quad(T \rightarrow \infty),
$$

where $w_{h}^{(T)}\left(\psi_{l l^{\prime}}\right)=\left\langle w_{h}^{(T)}, \psi_{l l^{\prime}}\right\rangle$ and $\left(\psi_{l l^{\prime}}: l, l^{\prime} \in \mathbb{N}\right)$ an orthonormal basis of $L^{2}\left([0,1]^{2}, \mathbb{C}\right)$ Proof. The proof is given in three parts, the first of which provides the outset, the second gives the arguments for the stationary case, while the third deals with the locally stationary situation.

(1) Preliminaries. Fix $\tau_{1}, \tau_{2} \in[0,1]$ and $h=1, \ldots, T-1$. It will be shown that the finite-dimensional distributions of $\left(w_{h}^{(T)}\left(\tau_{1}, \tau_{2}\right): T \in \mathbb{N}\right)$ converge to a Gaussian distribution by proving that the higher order cumulants of the terms $\sqrt{T} w_{h}^{(T)}\left(\psi_{l l^{\prime}}\right)=$ $\sqrt{T}\left\langle w_{h}^{(T)}, \psi_{l l^{\prime}}\right\rangle$ vanish asymptotically. To formulate this, consider an array of the form

$$
\begin{array}{cc}
(1,1) & (1,2) \\
\vdots & \vdots \\
(k, 1) & (k, 2)
\end{array}
$$

and let the value $s=i i^{\prime}$ correspond to entry $\left(i, i^{\prime}\right)$. For a partition $P=\left\{P_{1}, \ldots, P_{Q}\right\}$, the elements of a set $P_{q}$ will be denoted by $s_{q 1}, \ldots, s_{q m_{q}}$ where $\left|P_{q}\right|=m_{q}$ is the corresponding number of elements in $P_{q}$. Associate with entry $s$ the frequency index $j_{s}=j_{i i^{\prime}}=(-1)^{i^{\prime}-1}\left(j_{i}+h_{i}^{i^{\prime}-1}\right)$, Fourier frequency $\lambda_{j_{s}}=\frac{2 \pi j_{s}}{T}$ and the basis function index $v_{s}=v_{i i^{\prime}}=l_{i}^{2-i^{\prime}} l_{i}^{i^{\prime}-1}$ for $i=1, \ldots, k$ and $i^{\prime}=1$, 2. An application of the product theorem for cumulants yields

$$
\begin{aligned}
\operatorname{cum} & \left(\sum_{j_{1}=1}^{T} D_{\omega_{j_{1}}}^{\left(l_{1}\right)} D_{-\omega_{j_{1}+h_{1}}}^{\left(l_{1}^{\prime}\right)}, \ldots, \sum_{j_{k}=1}^{T} D_{\omega_{j_{k}}}^{\left(l_{k}\right)} D_{-\omega_{j_{k}+h_{k}}}^{\left(\left(l_{k}^{\prime}\right)\right.}\right) \\
& =\sum_{j_{1}, \ldots, j_{k}} \sum_{i . p .} \operatorname{cum}\left(D_{\lambda_{j_{s}}}^{\left(v_{s}\right)}: s \in P_{1}\right) \cdots \operatorname{cum}\left(D_{\lambda_{j_{s}}}^{\left(v_{s}\right)}: s \in P_{Q}\right),
\end{aligned}
$$

where the summation extends over all indecomposable partitions $P=\left\{P_{1}, \ldots, P_{Q}\right\}$ of (4.48). Because $X_{t}$ has zero-mean, the number of elements within each set must satisfy $m_{q} \geqslant 2$. To ease notation, write $D_{\omega_{j_{k}}}^{(l)}=\left\langle D_{\omega_{j_{k}}}^{(T)}, \psi_{l}\right\rangle$ and

$$
\left(\mathcal{F}_{t / T ; \lambda_{j s}}^{\left(v_{s}\right)}: s \in P_{q}\right)=\left\langle f_{t / T ; \lambda_{j q 1}, \ldots, \lambda_{j_{q m_{q}-1}}}, \bigotimes_{i^{\prime}=1}^{m_{q}} \psi_{v_{s_{q i^{\prime}}}}\right\rangle,
$$

noting that the latter quantity is well-defined. An application of Lemma B.1.2 yields

$$
\begin{aligned}
\sum_{i . p .} & \prod_{q=1}^{Q} \operatorname{cum}\left(D_{\lambda_{j_{s}}}^{\left(v_{s}\right)}: s \in P_{q}\right) \\
& =\sum_{i . p .} \prod_{q=1}^{Q}\left[\frac{(2 \pi)^{m_{q} / 2-1}}{T^{m_{q} / 2}}\left(\sum_{t=0}^{T-1} \mathcal{F}_{t / T ; \lambda_{j}}^{\left(v_{s}\right)} e^{-\mathrm{i} \sum_{s} t \lambda_{j_{s}}}: s \in P_{q}\right)+O\left(\frac{1}{T^{m_{q} / 2}}\right)\right],
\end{aligned}
$$


where under the null $\mathcal{F}_{t / T ; \lambda_{j}}^{\left(v_{s}\right)}=\mathcal{F}_{\lambda_{j_{s}}}^{\left(v_{s}\right)}$. In the following, the proof is separated into the cases where the true process is stationary and where it is locally stationary.

(2) Proof under stationarity. Recall that $\sup _{\boldsymbol{\omega}}\left\|\mathcal{F}_{\omega_{j_{1}}, \ldots, \omega_{j_{k^{\prime}-1}}}\right\| 2<\infty$ for all $k^{\prime} \leqslant$ $k$, and thus by the Cauchy-Schwarz Inequality $\sup _{\boldsymbol{\omega}}\left|\mathcal{F}_{\lambda_{j_{s}}}^{\left(v_{s}\right)}\right|<\infty$ for $s \in P_{q}$ and $q=1, \ldots, Q$. Therefore,

$$
\begin{aligned}
& \sum_{i . p .} \prod_{q=1}^{Q} \operatorname{cum}\left(D_{\lambda_{j_{s}}}^{\left(v_{s}\right)}: s \in P_{q}\right) \\
& \quad \leqslant \sum_{i . p .} \prod_{q=1}^{Q}\left[\frac{(2 \pi)^{m_{q} / 2-1} K_{q}}{T^{m_{q} / 2}} \Delta^{(T)}\left(\sum_{s \in P_{q}} \lambda_{j_{s}}\right)+O\left(\frac{1}{T^{m_{q} / 2}}\right)\right]
\end{aligned}
$$

for some constants $K_{1}, \ldots, K_{Q}$ independent of $T$. Due to the functions $\Delta^{(T)}$, there are $Q$ constraints if $Q<k$ or if $Q=k$ and there exists $h_{i_{1}}$ and $h_{i_{2}}$ such that $h_{i_{1}} \neq h_{i_{2}}$ for $i_{1}, i_{2} \in\{1, \ldots, k\}$. On the other hand, if the size of the partition is equal to $k$ and $h_{i_{1}}=h_{i_{2}}$ for all $i_{1}, i_{2} \in\{1, \ldots, k\}$, there are $Q-1$ constraints. This implies that

$$
\begin{aligned}
\frac{1}{T^{n / 2}} & \operatorname{cum}\left(\sum_{j_{1}=1}^{T} D_{\omega_{j_{1}}}^{\left(l_{1}\right)} D_{-\omega_{j_{1}+h_{1}}}^{\left(l_{1}^{\prime}\right)}, \ldots, \sum_{j_{n}=1}^{T} D_{\omega_{j_{k}}}^{\left(l_{k}\right)} D_{-\omega_{j_{k}+h_{k}}}^{\left(l_{k}^{\prime}\right)}\right) \\
& =O\left(T^{-n / 2} T^{n-(M-1)} T^{-2 n / 2} T^{M}\right) \\
& =O\left(T^{-n / 2+1}\right) .
\end{aligned}
$$

The cumulants of order $k \geqslant 3$ will therefore tend to 0 as $T \rightarrow \infty$.

(3) Proof under local stationarity. Write (4.49) in terms of the Fourier coefficients as

$$
\begin{aligned}
& \frac{1}{T^{n / 2}} \sum_{j_{1}, \ldots, j_{k}=1}^{T} \sum_{i . p .} \prod_{q=1}^{Q} \operatorname{cum}\left(D_{\lambda_{k_{s}}}^{\left(v_{s}\right)}: s \in P_{q}\right) \\
& \quad=\frac{1}{T^{n / 2}} \sum_{j_{1}, \ldots, j_{k}=1}^{T} \sum_{i . p .} \prod_{q=1}^{Q}\left[\frac{(2 \pi)^{m_{q} / 2-1}}{T^{m_{q} / 2-1}}\left(\tilde{\mathcal{F}}_{\sum_{s} j_{s} \lambda_{j_{s}}}^{\left(v_{s}\right)}: s \in P_{q}\right)+O\left(\frac{1}{T^{m_{q} / 2}}\right)\right] .
\end{aligned}
$$

Note that, by Corollary B.1.1 and the Cauchy-Schwarz Inequality,

$$
\sum_{j=1}^{T}\left|\tilde{\mathcal{F}}_{\sum_{s} j_{s} ; \lambda_{j s}}^{\left(v_{s}\right)}\right| \leqslant \sup _{\omega} \sum_{j \in \mathbb{Z}}\left\|\tilde{\mathcal{F}}_{j ; \omega}\right\|_{2} \prod_{i=1}^{m_{q}}\left\|\psi_{v_{q i}}\right\|_{2}<\infty, \quad s \in P_{q},
$$

for all $q=1, \ldots, Q$. If $Q<k$ or if $Q=k$ and there are $h_{i_{1}}$ and $h_{i_{2}}$ such that $h_{i_{1}} \neq h_{i_{2}}$ for $i_{1}, i_{2} \in\{1, \ldots, k\}$ within the same set, then there is dependence on $Q$ of the $k$ sums $j_{1}, \ldots, j_{n}$. On the other hand, if the size of the partition is equal to $k$ and $h_{i_{1}}=h_{i_{2}}$ for all $i_{1}, i_{2}=1, \ldots, k$, then there are $Q-1$ constraints on $j_{1}, \ldots, j_{n}$. Thus, similar to the stationary case, it follows that the order is

$$
O\left(T^{-k / 2} T^{k-Q+1} T^{-2 k / 2+Q}\right)=O\left(T^{-k / 2+1}\right),
$$

hence giving the result. 


\section{B.4 Weak convergence}

In this section, the asymptotic properties of the statistic $\hat{\gamma}^{(T)}$ are derived. Theorems 4.4.2 and 4.4.8 imply that for appropriate choices of the bandwidths the analysis of distributional properties may be restricted to $\gamma^{(T)}$ under both hypotheses. Because the denominator of $\gamma^{(T)}$ is deterministic, the analysis may be further simplified by focusing on the properties of the numerator

$$
w_{h}^{(T)}\left(\tau_{1}, \tau_{2}\right)=\frac{1}{T} \sum_{j=1}^{T} D_{\omega_{j}}^{(T)}\left(\tau_{1}\right) D_{-\omega_{j+h}}^{(T)}\left(\tau_{2}\right) .
$$

To demonstrate weak convergence, it is useful to apply a result from Cremers and Kadelka (1986) as it considerably weakens the verification of the usual tightness condition often invoked in weak convergence proofs. In particular, the following lemma indicates that weak convergence of the functional process will almost directly follow from the weak convergence of the finite dimensional distributions once it is weakly tight in a certain sense.

Lemma B.4.1. Let $(T, \mathcal{B}, \mu)$ be a measure space, let $(E,|\cdot|)$ be a Banach space, and let $X=\left(X_{n}: n \in \mathbb{N}\right)$ be a sequence of random elements in $L_{E}^{p}(T, \mu)$ such that

(i) the finite-dimensional distributions of $X$ converge weakly to those of a random element $X_{0}$ in $L_{E}^{p}(T, \mu)$;

(ii) $\limsup _{n \rightarrow \infty} \mathbb{E}\left[\left\|X_{n}\right\|_{p}^{p}\right] \leqslant \mathbb{E}\left[\left\|X_{0}\right\|_{p}^{p}\right]$.

Then, $X$ converges weakly to $X_{0}$ in $L_{E}^{p}(T, \mu)$.

To apply the lemma in the present context, consider the sequence $\left(\hat{E}_{r}^{(T)}\left(\tau_{1}, \tau_{2}\right): T \in\right.$ $\mathbb{N})$ of random elements in $L^{2}\left([0,1]^{2}, \mathbb{C}\right)$, for $\tau, \tau^{\prime} \in[0,1]$ and $h=1, \ldots, T-1$ defined through

$$
\hat{E}_{h}^{(T)}\left(\tau_{1}, \tau_{2}\right)=\sqrt{T}\left(w_{h}^{(T)}\left(\tau_{1}, \tau_{2}\right)-\mathbb{E}\left[w_{h}^{(T)}\left(\tau_{1}, \tau_{2}\right)\right]\right) .
$$

Let $\left(\psi_{l}: l \in \mathbb{N}\right)$ be an orthonormal basis of $H_{\mathbb{C}}$ and denote the elementary tensor product by $\psi_{l l^{\prime}}=\psi_{l} \otimes \psi_{l}^{\prime}$. Then $\left(\psi_{l l^{\prime}}: l, l^{\prime} \in \mathbb{N}\right)$ forms an orthonormal basis of $L^{2}\left([0,1]^{2}, \mathbb{C}\right)$ and a basis expansion yields

$$
\hat{E}_{h}^{(T)}=\sum_{l, l^{\prime}=1}^{\infty}\left\langle\hat{E}_{h}^{(T)}, \psi_{l l^{\prime}}\right\rangle \psi_{l l^{\prime}}
$$

It can therefore be seen that the finite-dimensional distributions of the basis coefficients provide a complete characterization of the distributional properties of $\hat{E}_{r}^{(T)}$ : Weak convergence of $\left(\left\langle\hat{E}_{h}^{(T)}, \psi_{l l^{\prime}}\right\rangle: l, l^{\prime} \in \mathbb{N}\right)$ in the sequence space $\ell_{\mathbb{C}}^{2}$ will imply weak convergence of the process $\left(\hat{E}_{r}^{(T)}\left(\tau_{1}, \tau_{2}\right): T \in \mathbb{N}\right)$. Identifying the functional $\hat{E}_{h}^{(T)}$ with its the dual $\left(\hat{E}_{h}^{(T)}\right)^{*} \in L^{2}\left([0,1]^{2}, \mathbb{C}\right)^{*}$, leads to the pairing

$$
\hat{E}_{h}^{(T)}(\phi)=\left\langle\hat{E}_{h}^{(T)}, \phi\right\rangle
$$


for all $\phi \in L^{2}\left([0,1]^{2}, \mathbb{C}\right)^{*}$. The second condition of Lemma B.4.1 will hence be satisfied if

$$
\mathbb{E}\left[\left\|\hat{E}_{h}^{(T)}\right\|_{2}^{2}\right]=\sum_{l, l^{\prime}=1}^{\infty} \mathbb{E}\left[\left|\hat{E}_{h}^{(T)}\left(\psi_{l l^{\prime}}\right)\right|^{2}\right] \rightarrow \sum_{l, l^{\prime}=1}^{\infty} \mathbb{E}\left[\left|E_{h}\left(\psi_{l l^{\prime}}\right)\right|^{2}\right]=\mathbb{E}\left[\left\|E_{h}\right\|_{2}^{2}\right] \quad(T \rightarrow \infty),
$$

with $E_{h}$ denoting the limiting process. The following theorem shows that the finite dimensional distributions converge weakly to a Gaussian process and is a restatement of the results of Section B.3.

Theorem B.4.2. Under Assumption 4.4.1, for all $l_{i}, l_{i}^{\prime} \in \mathbb{N}, h_{i}=1, \ldots, T-1$, $i=1, \ldots, k$ and $k \geqslant 3$,

$$
\operatorname{cum}\left(\hat{E}_{r_{1}}^{(T)}\left(\psi_{l_{1} l_{1}^{\prime}}\right), \ldots, \hat{E}_{h_{k}}^{(T)}\left(\psi_{l_{k} l_{k}^{\prime}}\right)\right)=o(1) \quad(T \rightarrow \infty) .
$$

The higher order cumulant result of Theorem B.4.2 establishes, for all $q \in \mathbb{N}$, the joint convergence of $\hat{E}_{h}^{(T)}\left(\psi_{l_{1} l_{1}^{\prime}}\right), \ldots, \hat{E}_{h}^{(T)}\left(\psi_{l_{q} l_{q}^{\prime}}\right)$ and condition (i) of Lemma B.4.1 is therefore satisfied. Weak convergence of the functional process can now be determined, distinguishing between the real and imaginary parts.

Theorem B.4.3 (Weak convergence under the null). Let $\left(X_{t}: t \in \mathbb{Z}\right)$ be $a$ stochastic process taking values in $H_{\mathbb{R}}$ satisfying Assumption 4.4 .1 with $k=8$ and $\ell=2$. Then,

$$
\left(\Re \hat{E}_{h}^{(T)}, \Im \hat{E}_{h}^{(T)}: h=1, \ldots, T-1\right) \stackrel{d}{\rightarrow}\left(\mathcal{R}_{h}, \mathcal{I}_{h}: h=1, \ldots, T-1\right),
$$

where $\mathcal{R}_{h}, \mathcal{I}_{h}, h=1, \ldots, T-1$, are jointly Gaussian elements in $L^{2}\left([0,1]^{2}, \mathbb{C}\right)$ with means $\mathbb{E}\left[\mathcal{R}_{h}\left(\psi_{l l^{\prime}}\right)\right]=\mathbb{E}\left[\mathcal{I}_{h}\left(\psi_{l l^{\prime}}\right)\right]=0$ and covariances

$$
\begin{aligned}
\operatorname{cov}\left(\mathcal{R}_{h_{1}}\left(\psi_{l_{1} l_{1}^{\prime}}\right), \mathcal{R}_{h_{2}}\left(\psi_{l_{2} l_{2}}\right)\right) \\
=\operatorname{cov}\left(\mathcal{I}_{h_{1}}\left(\psi_{l_{1} l_{1}^{\prime}}\right), \mathcal{I}_{h_{2}}\left(\psi_{l_{2} l_{2}^{\prime}}\right)\right) \\
=\frac{1}{4 \pi} \int_{0}^{2 \pi}\left\langle\mathcal{F}_{\omega}\left(\psi_{l_{2}}\right), \psi_{l_{1}}\right\rangle\left\langle\mathcal{F}_{-\omega-\omega_{h}}\left(\psi_{l_{2}^{\prime}}\right), \psi_{l_{1}}\right\rangle d \omega \\
+\frac{1}{4 \pi} \int_{0}^{2 \pi}\left\langle\mathcal{F}_{\omega}\left(\psi_{l_{2}^{\prime}}\right), \psi_{l_{1}}\right\rangle\left\langle\mathcal{F}_{-\omega-\omega_{h}}\left(\psi_{l_{2}}\right), \psi_{l_{1}^{\prime}}\right\rangle d \omega \\
\quad+\frac{1}{4 \pi} \int_{0}^{2 \pi} \int_{0}^{2 \pi}\left\langle\mathcal{F}_{\omega,-\omega-\omega_{h},-\omega^{\prime}}\left(\psi_{l_{2} l_{2}^{\prime}}\right), \psi_{l_{1} l_{1}^{\prime}}\right\rangle d \omega d \omega^{\prime}
\end{aligned}
$$

for all $h_{1}=h_{2}$ and $l_{1}, l_{1}^{\prime}, l_{2}, l_{2}^{\prime}$, and 0 otherwise. In addition,

$$
\operatorname{cov}\left(\mathcal{R}_{h_{1}}\left(\psi_{l_{1} l_{1}^{\prime}}\right), \mathcal{I}_{h_{2}}\left(\psi_{l_{2} l_{2}^{\prime}}\right)\right)=0
$$

uniformly in $h_{1}, h_{2}$ and $l_{1}, l_{1}^{\prime}, l_{2}, l_{2}^{\prime}$.

Proof. The covariance structure follows from Theorem 4.4 .4 and the convergence of the finite-dimensional distributions from Theorem B.4.2. It then remains to verify 
that the condition (ii) of Lemma B.4.1 is satisfied. This follows from Theorem 4.4.4, since

$$
\mathbb{E}\left[\left\|\hat{E}_{h}^{(T)}\right\|_{2}^{2}\right]=\int_{[0,1]^{2}} \operatorname{var}\left(\hat{E}_{h}^{(T)}\left(\tau, \tau^{\prime}\right)\right) d \tau d \tau^{\prime}=T\left\|\operatorname{var}\left(w_{h}^{(T)}\right)\right\|_{2}^{2}=2\left\|\operatorname{var}\left(\mathcal{R}_{h}\right)\right\|_{2}^{2} .
$$

This completes the proof.

Under the alternative, we obtain a similar result:

Theorem B.4.4 (Weak convergence under the alternative). Let $\left(X_{t}: t \in \mathbb{Z}\right)$ be a stochastic process taking values in $H_{\mathbb{R}}$ satisfying Assumption 4.4 .6 with $k=8$ and $\ell=2$. Then,

$$
\left(\Re \hat{E}_{h}^{(T)}, \Im \hat{E}_{h}^{(T)}: h=1, \ldots, T-1\right) \stackrel{d}{\rightarrow}\left(\mathcal{R}_{h}, \mathcal{I}_{h}: h=1, \ldots, T-1\right),
$$

where $\mathcal{R}_{h}, \mathcal{I}_{h}, h=1, \ldots, T-1$, are jointly Gaussian elements in $L^{2}\left([0,1]^{2}, \mathbb{C}\right)$ with means $\mathbb{E}\left[\mathcal{R}_{h}\left(\psi_{l l^{\prime}}\right)\right]=\mathbb{E}\left[\mathcal{I}_{h}\left(\psi_{l l^{\prime}}\right)\right]=0$ and covariance structure

1. $\operatorname{cov}\left(\mathcal{R}_{h_{1}}\left(\psi_{l_{1} l_{1}^{\prime}}\right), \mathcal{R}_{h_{2}}\left(\psi_{l_{2} l_{2}^{\prime}}\right)\right)=$

$$
\frac{1}{4}\left[\Upsilon_{h_{1}, h_{2}}\left(\psi_{l_{1} l_{1}^{\prime} l_{2} l_{2}^{\prime}}\right)+\dot{\Upsilon}_{h_{1}, h_{2}}\left(\psi_{l_{1} l_{1}^{\prime} l_{2} l_{2}^{\prime}}\right)+\grave{\Upsilon}_{h_{1}, h_{2}}\left(\psi_{l_{1} l_{1}^{\prime} l_{2} l_{2}^{\prime}}\right)+\bar{\Upsilon}_{h_{1}, h_{2}}\left(\psi_{l_{1} l_{1}^{\prime} l_{2} l_{2}^{\prime}}\right)\right]
$$

2. $\operatorname{cov}\left(\mathcal{R}_{h_{1}}\left(\psi_{l_{1} l_{1}^{\prime}}\right), \mathcal{I}_{h_{2}}\left(\psi_{l_{2} l_{2}}\right)\right)=$

$$
\frac{1}{4 \mathrm{i}}\left[\Upsilon_{h_{1}, h_{2}}\left(\psi_{l_{1} l_{1}^{\prime} l_{2} l_{2}^{\prime}}\right)-\dot{\Upsilon}_{h_{1}, h_{2}}\left(\psi_{l_{1} l_{1}^{\prime} l_{2} l_{2}^{\prime}}\right)+\grave{\Upsilon}_{h_{1}, h_{2}}\left(\psi_{l_{1} l_{1}^{\prime} l_{2} l_{2}^{\prime}}\right)-\bar{\Upsilon}_{h_{1}, h_{2}}\left(\psi_{l_{1} l_{1}^{\prime} l_{2} l_{2}^{\prime}}\right)\right]
$$

3. $\operatorname{cov}\left(\mathcal{I}_{h_{1}}\left(\psi_{l_{1} l_{1}^{\prime}}\right), \mathcal{I}_{h_{2}}\left(\psi_{l_{2} l_{2}^{\prime}}\right)\right)=$

$$
\frac{1}{4}\left[\Upsilon_{h_{1}, h_{2}}\left(\psi_{l_{1} l_{1}^{\prime} l_{2} l_{2}^{\prime}}\right)-\dot{\Upsilon}_{h_{1}, h_{2}}\left(\psi_{l_{1} l_{1}^{\prime} l_{2} l_{2}^{\prime}}\right)-\grave{\Upsilon}_{h_{1}, h_{2}}\left(\psi_{l_{1} l_{1}^{\prime} l_{2} l_{2}^{\prime}}\right)+\bar{\Upsilon}_{h_{1}, h_{2}}\left(\psi_{l_{1} l_{1}^{\prime} l_{2} l_{2}^{\prime}}\right)\right]
$$

for all $h_{1}, h_{2}$ and $l_{1}, l_{1}^{\prime}, l_{2}, l_{2}^{\prime}$, and where $\Upsilon_{h_{1}, h_{2}}, \dot{\Upsilon}_{h_{1}, h_{2}}, \grave{\Upsilon}_{h_{1}, h_{2}}$ and $\bar{\Upsilon}_{h_{1}, h_{2}}$ are given in (4.71)-(4.73).

Proof. The covariance structure follows along the lines of Theorem 4.4.10 and the convergence of the finite-dimensional distributions from Theorem B.4.2. Condition (ii) of Lemma B.4.1 is satisfied by Theorem 4.4 .10 since

$\mathbb{E}\left[\left\|\hat{E}_{h}^{(T)}\right\|_{2}^{2}\right]=\int_{[0,1]^{2}} \operatorname{var}\left(\hat{E}_{h}^{(T)}\left(\tau, \tau^{\prime}\right)\right) d \tau d \tau^{\prime}=T\left\|\operatorname{var}\left(w_{h}^{(T)}\right)\right\|_{2}^{2}=\left\|\operatorname{var}\left(\mathcal{R}_{h}\right)\right\|_{2}^{2}+\left\|\operatorname{var}\left(\mathcal{I}_{h}\right)\right\|_{2}^{2}$,

which completes the proof.

\section{B.5 Proofs under the null hypothesis of station- arity}

Let $\left(\psi_{l}: l \in \mathbb{N}\right)$ be an orthonormal basis of $H_{\mathbb{C}}$. Then $\left(\psi_{l_{1}} \otimes \cdots \otimes \psi_{l_{k}}: l_{1}, \ldots, l_{k} \in \mathbb{N}\right)$ is an orthonormal basis of $H_{\mathbb{C}}^{k}$. To ease notation, denote the elementary tensor 
$\psi_{l l^{\prime}}=\psi_{l} \otimes \psi_{l^{\prime}}$. Write $D_{\omega}^{(l)}=\left\langle D_{\omega}^{(T)}, \psi_{l}\right\rangle, \mathcal{F}_{\omega}^{\left(l, l^{\prime}\right)}=\left\langle\mathcal{F}_{\omega}\left(\psi_{l^{\prime}}\right), \psi_{l}\right\rangle$ and $\mathcal{F}_{\omega_{j_{1}}, \omega_{j_{2}}, \omega_{j_{3}}}^{\left(l, m^{\prime} m^{\prime}\right)}=$ $\left\langle\mathcal{F}_{\omega_{j_{1}}, \omega_{j_{2}}, \omega_{j_{3}}}\left(\psi_{l^{\prime} m^{\prime}}\right), \psi_{l m}\right\rangle$. Recall that the sequence $\left(D_{\omega_{j}}^{(T)}\right)$ has mean zero. Ignoring the denominator for the moment, Theorem B.1.1 leads to the following covariance structure of the fDfT's:

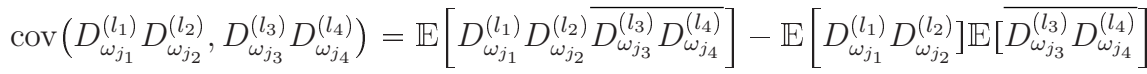

$$
\begin{aligned}
& =\operatorname{cum}\left(D_{\omega_{j_{1}}}^{\left(l_{1}\right)}, D_{\omega_{j_{2}}}^{\left(l_{2}\right)}, \overline{D_{\omega_{j_{3}}}^{\left(l_{3}\right)}, D_{\omega_{j_{4}}}^{\left(l_{4}\right)}}\right) \\
& +\operatorname{cum}\left(D_{\omega_{j_{1}}}^{\left(l_{1}\right)}, \overline{D_{\omega_{j_{3}}}^{\left(l_{3}\right)}}\right) \operatorname{cum}\left(D_{\omega_{j_{2}}}^{\left(l_{2}\right)}, \overline{D_{\omega_{j_{4}}}^{\left(l_{4}\right)}}\right) \\
& +\operatorname{cum}\left(D_{\omega_{j_{1}}}^{\left(l_{1}\right)}, \overline{D_{\omega_{j_{4}}}^{\left(l_{4}\right)}}\right) \operatorname{cum}\left(D_{\omega_{j_{2}}}^{\left(l_{2}\right)}, \overline{D_{\omega_{j_{3}}}^{\left(l_{3}\right)}}\right)
\end{aligned}
$$

for $l_{1}, l_{2}, l_{3}, l_{4} \in \mathbb{N}$. In particular,

$$
\begin{aligned}
\operatorname{cov}\left(D_{\omega_{j_{1}}}^{\left(l_{1}\right)} D_{-\omega_{j_{1}+h_{1}}}^{\left(l_{2}\right)}, D_{\omega_{j_{2}}}^{\left(l_{3}\right)} D_{-\omega_{j_{2}+h_{2}}}^{\left(l_{4}\right)}\right)= & \operatorname{cum}\left(D_{\omega_{j_{1}}}^{\left(l_{1}\right)}, D_{-\omega_{j_{1}+h_{1}}}^{\left(l_{2}\right)}, D_{-\omega_{j_{2}}}^{\left(l_{2}\right)}, D_{\omega_{\omega_{2}+h_{2}}}^{\left(l_{4}\right)}\right) \\
& +\operatorname{cum}\left(D_{\omega_{j_{1}}}^{\left(l_{1}\right)}, D_{-\omega_{j_{2}}}^{\left(l_{3}\right)}\right) \operatorname{cum}\left(D_{-\omega_{j_{1}+h_{1}}}^{\left(l_{2}\right)}, D_{\omega_{j_{2}}+h_{2}}^{\left(l_{4}\right)}\right) \\
& +\operatorname{cum}\left(D_{\omega_{j_{1}}}^{\left(l_{1}\right)}, D_{\omega_{j_{2}+h_{2}}}^{\left(l_{4}\right)}\right) \operatorname{cum}\left(D_{-\omega_{j_{1}+h_{1}}}^{\left(l_{2}\right)}, D_{-\omega_{j_{2}}}^{\left(l_{2}\right)}\right) .
\end{aligned}
$$

Given the underlying assumptions are satisfied for $k=4$, Lemma B.1.2 implies this can be written as

$$
\begin{aligned}
& \frac{(2 \pi)}{T^{2}} \sum_{t=1}^{T} \mathcal{F}_{t / T ; \omega_{1},-\omega_{j_{1}+h_{1}},-\omega_{j_{2}}}^{\left(l_{1} l_{2}, l_{3} l_{4}\right)} e^{-\mathrm{i} t\left(\omega_{h_{2}}-\omega_{h_{1}}\right)}+O\left(\frac{1}{T^{2}}\right) \\
& +\left[\frac{1}{T} \sum_{t=1}^{T} \mathcal{F}_{t / T, \omega_{j_{1}}}^{\left(l_{1}, l_{3}\right)} e^{-\mathrm{i} t\left(\omega_{j_{1}}-\omega_{j_{2}}\right)}+O\left(\frac{1}{T}\right)\right]\left[\frac{1}{T} \sum_{s=1}^{T} \mathcal{F}_{s / T,-\omega_{j_{1}+h_{1}}}^{\left(l_{2}, l_{4}\right)} e^{-\mathrm{i} s\left(-\omega_{j_{1}+h_{1}}+\omega_{j_{2}+h_{2}}\right)}+O\left(\frac{1}{T}\right)\right] \\
& +\left[\frac{1}{T} \sum_{t=1}^{T} \mathcal{F}_{t / T, \omega_{j_{1}}}^{\left(l_{1}, l_{4}\right)} e^{-\mathrm{i} t\left(\omega_{j_{1}}+\omega_{j_{2}+h_{2}}\right)}+O\left(\frac{1}{T}\right)\right]\left[\frac{1}{T} \sum_{s=1}^{T} \mathcal{F}_{s / T,-\omega_{j_{1}+h_{1}}}^{\left(l_{2}, l_{3}\right)} e^{-\mathrm{i} s\left(-\omega_{j_{1}+h_{1}}-\omega_{j_{2}}\right)}+O\left(\frac{1}{T}\right)\right] .
\end{aligned}
$$

Proof of Theorem 4.4.4. By Lemma B.1.2, for $h=1, \ldots, T-1$,

$$
\mathbb{E}\left[\sqrt{T} \gamma_{h}^{(T)}\left(l, l^{\prime}\right)\right]=\frac{1}{\sqrt{T}} \sum_{j=1}^{T}\left[\frac{\mathcal{F}_{\omega_{j}}^{\left(l, l^{\prime}\right)}}{\left.\left(\mathcal{F}_{\omega_{j}}^{(l, l)} \mathcal{F}_{\omega_{j+h}}^{\left(l^{\prime}, l^{\prime}\right)}\right)\right)^{1 / 2}} \frac{1}{T} \Delta^{(T)}(h)+O\left(\frac{1}{T}\right)\right]=O\left(\frac{1}{\sqrt{T}}\right) .
$$

For the covariance structure under the null hypothesis, use that (4.57) becomes

$$
\begin{aligned}
& \frac{1}{T} \sum_{j_{1}, j_{2}=1}^{T}\left(\frac{(2 \pi)}{T^{2}} \mathcal{F}_{\omega_{j_{1}},-\omega_{j_{1}+h_{1}},-\omega_{j_{2}}}^{\left(l_{1} l_{2}, l_{2} l_{4}\right)} \Delta_{T}^{\left(\omega_{h_{2}}-\omega_{h_{1}}\right)}+O\left(\frac{1}{T^{2}}\right)\right. \\
& +\left[\mathcal{F}_{\omega_{j_{1}}}^{\left(l_{1}, l_{3}\right)} \frac{1}{T} \Delta_{T}^{\left(\omega_{j_{1}}-\omega_{j_{2}}\right)}+O\left(\frac{1}{T}\right)\right]\left[\mathcal{F}_{-\omega_{j_{1}+h_{1}}}^{\left(l_{2}, l_{4}\right)} \frac{1}{T} \Delta_{T}^{\left(\omega_{j_{1}+h_{1}}-\omega_{j_{2}+h_{2}}\right)}+O\left(\frac{1}{T}\right)\right] \\
& \left.+\left[\mathcal{F}_{\omega_{j_{1}}}^{\left(l_{1}, l_{4}\right)} \frac{1}{T} \Delta_{T}^{\left(\omega_{j_{1}}+\omega_{j_{2}+h_{2}}\right)}+O\left(\frac{1}{T}\right)\right]\left[\mathcal{F}_{-\omega_{j_{1}+h_{1}}}^{\left(l_{2}, l_{3}\right)} \frac{1}{T} \Delta_{T}^{\left(-\omega_{j_{1}+h_{1}}-\omega_{j_{2}}\right)}+O\left(\frac{1}{T}\right)\right]\right) .
\end{aligned}
$$


In case $h_{1} \neq h_{2}$, the first line is of order $O\left(T^{-1}\right)$. In the second and third line, it can be seen that the cross terms and final terms are of order $O\left(T^{-1}\right)$ uniformly in $\omega_{j}$. The first product term in the second line will only be of order $O(1)$ if $j_{1}=j_{2}$ and $j_{1}+h_{1}=j_{2}+h_{2}$, while in the third line this requires $j_{1}=-j_{2}-h_{2}$ and $j_{2}=-j_{1}-h_{1}$. Therefore,

$$
\begin{aligned}
& \operatorname{cov}\left(\frac{1}{\sqrt{T}} \sum_{j_{1}=1}^{T} D_{\omega_{1}}^{\left(l_{1}\right)} D_{-\omega_{j_{1}+h_{1}}}^{\left(l_{2}\right)}, \frac{1}{\sqrt{T}} \sum_{j_{2}=1}^{T} D_{\omega_{j_{2}}}^{\left(l_{3}\right)} D_{-\omega_{j_{2}+h_{2}}}^{\left(l_{4}\right)}\right) \\
& = \begin{cases}\frac{1}{T} \sum_{j_{1}=1}^{T}\left(\mathcal{F}_{\omega_{j_{1}}}^{\left(l_{1}, l_{3}\right)} \mathcal{F}_{-\omega_{j_{1}+h}}^{\left(l_{2}, l_{4}\right)}+\mathcal{F}_{\omega_{j_{1}}}^{\left(l_{1}, l_{4}\right)} \mathcal{F}_{-\omega_{j_{1}+h}}^{\left(l_{2}, l_{3}\right)}\right) & \\
+\frac{2 \pi}{T^{2}} \sum_{j_{1}, j_{2}=1}^{T} \mathcal{F}_{\omega_{j_{1}},-\omega_{j_{1}+h},-\omega_{j_{2}}}^{\left(l_{1} l_{2}, l_{3} l_{l}\right)}, & \text { if } h_{1}=h_{2}=h, \\
O\left(\frac{1}{T}\right), & \text { if } h_{1} \neq h_{2},\end{cases}
\end{aligned}
$$

while the covariance of the complex conjugate is given by

$$
\begin{aligned}
& \operatorname{cov}\left(\frac{1}{\sqrt{T}} \sum_{j_{1}=1}^{T} D_{-\omega_{1}}^{\left(l_{1}\right)} D_{\omega_{j_{1}+h_{1}}}^{\left(l_{2}\right)}, \frac{1}{\sqrt{T}} \sum_{j_{2}=1}^{T} D_{-\omega_{j_{2}}}^{\left(l_{3}\right)} D_{\omega_{j_{2}+h_{2}}}^{\left(l_{4}\right)}\right) \\
& = \begin{cases}\frac{1}{T} \sum_{j_{1}=1}^{T}\left(\mathcal{F}_{-\omega_{j_{1}}}^{\left(l_{1}, l_{3}\right)} \mathcal{F}_{\omega_{j_{1}+h}}^{\left(l_{2}, l_{4}\right)}+\mathcal{F}_{\omega_{-j_{1}}}^{\left(l_{1}, l_{4}\right)} \mathcal{F}_{\omega_{j_{1}+h}}^{\left(l_{2}, l_{3}\right)}\right) \\
+\frac{2 \pi}{T^{2}} \sum_{j_{1}, j_{2}=1}^{T} \mathcal{F}_{-\omega_{j_{1}}, \omega_{j_{1}+h}, \omega_{j_{2}}}^{\left(l_{1} l_{2}, l_{3} l_{4}\right)}, & \text { if } h_{1}=h_{2}=h . \\
O\left(\frac{1}{T}\right), & \text { if } h_{1} \neq h_{2} .\end{cases}
\end{aligned}
$$

Similarly, $T \operatorname{cov}\left(\gamma_{h_{1}}^{(T)}\left(l_{1}, l_{2}\right), \overline{\gamma_{h_{1}}^{(T)}\left(l_{3}, l_{4}\right)}\right)=O\left(T^{-1}\right)$ for $h_{2} \neq T-h_{1}$. Since

$$
\Re \gamma_{h_{1}}^{(T)}\left(l_{1}, l_{2}\right)=\frac{1}{2}\left(\gamma_{h_{1}}^{(T)}\left(l_{1}, l_{2}\right)+\overline{\gamma_{h_{1}}^{(T)}\left(l_{1}, l_{2}\right)}\right)
$$

and

$$
\Im \gamma_{r_{1}}^{(T)}\left(l_{1}, l_{2}\right)=\frac{1}{2 \mathrm{i}}\left(\gamma_{h_{1}}^{(T)}\left(l_{1}, l_{2}\right)-\overline{\gamma_{h_{1}}^{(T)}\left(l_{1}, l_{2}\right)}\right),
$$

it follows that

$$
T \operatorname{cov}\left(\Re \gamma_{h_{1}}^{(T)}\left(l_{1}, l_{2}\right), \Im \gamma_{h_{2}}^{(T)}\left(l_{3}, l_{4}\right)\right)=O\left(T^{-1}\right)
$$

uniformly in $h_{1}, h_{2}$. All together, the above derivation yields

$$
\begin{aligned}
T \operatorname{cov}\left(\Re \gamma_{h_{1}}^{(T)}\left(l_{1}, l_{2}\right), \Re \gamma_{h_{2}}^{(T)}\left(l_{3}, l_{4}\right)\right) & =T \operatorname{cov}\left(\Im \gamma_{h_{1}}^{(T)}\left(l_{1}, l_{2}\right), \Im \gamma_{h_{2}}^{(T)}\left(l_{3}, l_{4}\right)\right) \\
& =\frac{T}{2} \operatorname{cov}\left(\gamma_{h_{1}}^{(T)}\left(l_{1}, l_{2}\right), \gamma_{h_{2}}^{(T)}\left(l_{3}, l_{4}\right)\right),
\end{aligned}
$$


which, for, $h_{1}=h_{2}=h$, is equal to

$$
\begin{aligned}
T \operatorname{cov}\left(\gamma_{h}^{(T)}\left(l_{1}, l_{2}\right), \gamma_{h}^{(T)}\left(l_{3}, l_{4}\right)\right) \\
=\frac{(2 \pi)}{T^{2}} \sum_{j_{1}, j_{2}=1}^{T} \frac{\mathcal{F}_{\omega_{j_{1}},-\omega_{j_{1}+h},-\omega_{j_{2}}}^{\left(l_{1} l_{2}, l_{2} l_{l}\right.}}{\left(\mathcal{F}_{\omega_{j_{1}}}^{\left(l_{1}, l_{1}\right)} \mathcal{F}_{-\omega_{j_{1}+h}}^{\left(l_{2}, l_{2}\right)} \mathcal{F}_{-\omega_{j_{2}}}^{\left(l_{3}, l_{3}\right)} \mathcal{F}_{\omega_{j_{2}+h}}^{\left(l_{4}, l_{4}\right)}\right)^{1 / 2}}+O\left(\frac{1}{T^{2}}\right) \\
+\frac{1}{T} \sum_{j=1}^{T}\left[\frac{\mathcal{F}_{\omega_{j}}^{\left(l_{1}, l_{3}\right)}}{\left(\mathcal{F}_{\omega_{j}}^{\left(l_{1}, l_{1}\right)} \mathcal{F}_{-\omega_{j}}^{\left(l_{3}, l_{3}\right)}\right)^{1 / 2}}+O\left(\frac{1}{T}\right)\right]\left[\frac{\mathcal{F}_{-\omega_{j+h}}^{\left(l_{2}, l_{4}\right)}}{\left(\mathcal{F}_{-\omega_{j+h}}^{\left(l_{2}, l_{2}\right)} f_{\omega_{j+h}}^{\left(l_{4}, l_{4}\right)}\right)^{1 / 2}}+O\left(\frac{1}{T}\right)\right] \\
+\frac{1}{T} \sum_{j=1}^{T}\left[\frac{\mathcal{F}_{\omega_{j}}^{X,\left(l_{1}, l_{4}\right)}}{\left(\mathcal{F}_{\omega_{j}}^{\left(l_{1}, l_{1}\right)} \mathcal{F}_{-\omega_{j}}^{\left(l_{4}, l_{4}\right)}\right)^{1 / 2}}+O\left(\frac{1}{T}\right)\right]\left[\frac{\mathcal{F}_{-\omega_{j+h}}^{\left(l_{2}, l_{3}\right)}}{\left(\mathcal{F}_{-\omega_{j+h}}^{\left(l_{2}, l_{2}\right)} f_{\omega_{j+h}}^{\left(l_{3}, l_{3}\right)}\right)^{1 / 2}}+O\left(\frac{1}{T}\right)\right] .
\end{aligned}
$$

If the spectra are Lipschitz, the covariance structure of $\sqrt{T} \gamma^{(T)}$ is thus given by

$$
\begin{aligned}
& T \operatorname{cov}\left(\Re \gamma_{h_{1}}^{(T)}\left(l_{1}, l_{2}\right), \Re \gamma_{h_{2}}^{(T)}\left(l_{3}, l_{4}\right)\right) \\
& =T \operatorname{cov}\left(\Im \gamma_{h_{1}}^{(T)}\left(l_{1}, l_{2}\right), \Im \gamma_{h_{2}}^{(T)}\left(l_{3}, l_{4}\right)\right) \\
& =\left\{\begin{array}{cc}
\frac{1}{4 \pi}\left(\int \frac{\mathcal{F}_{\omega}^{\left(l_{1}, l_{3}\right)} \mathcal{F}_{-\omega-\omega_{h}}^{\left(l_{2}, l_{4}\right)}}{\sqrt{\mathcal{F}_{\omega}^{\left(l_{1}, l_{1}\right)} \mathcal{F}_{-\omega}^{\left(l_{3}, l_{3}\right)} \mathcal{F}_{-\omega-\omega_{h}}^{\left(l_{2}, l_{2}\right)} \mathcal{F}_{\omega+\omega_{h}}^{\left(l_{4}, l_{4}\right)}}} d \omega+\int \frac{\mathcal{F}_{\omega}^{\left(l_{1}, l_{4}\right)} \mathcal{F}_{-\omega-\omega_{h}}^{\left(l_{2}, l_{3}\right)}}{\sqrt{\mathcal{F}_{\omega}^{\left(l_{1}, l_{1}\right)} \mathcal{F}_{-\omega}^{\left(l_{4}, l_{4}\right)} \mathcal{F}_{-\omega-\omega_{h}}^{\left(l_{2}, l_{2}\right)} \mathcal{F}_{\omega+\omega_{h}}^{\left(l_{3}, l_{3}\right)}}} d \omega\right. \\
\left.\quad+\iint \frac{\mathcal{F}_{\omega,-\omega-\omega_{h},-\lambda}^{\left(l_{1} l_{2}, l_{l} l_{4}\right)}}{\sqrt{\mathcal{F}_{\omega}^{\left(l_{1}, l_{1}\right)} \mathcal{F}_{-\omega-\omega_{h}}^{\left(l_{2}, l_{2}\right)} \mathcal{F}_{-\omega^{\prime}}^{\left(l_{3}, l_{3}\right)} \mathcal{F}_{\omega^{\prime}+\omega_{h}}^{\left(l_{4}, l_{4}\right)}}} d \omega d \omega^{\prime}\right) & \text { if } h_{1}=h_{2}=h, \\
O\left(\frac{1}{T}\right) & \text { if } h_{1} \neq h_{2} .
\end{array}\right.
\end{aligned}
$$

Furthermore, $T \operatorname{cov}\left(\Re \gamma_{h_{1}}^{(T)}\left(l_{1}, l_{2}\right), \Im \gamma_{h_{2}}^{(T)}\left(l_{3}, l_{4}\right)\right)=O\left(T^{-1}\right)$ uniformly in $h_{1}, h_{2} \in \mathbb{Z}$. The above result implies in particular for the variance that

$$
\begin{aligned}
& T \operatorname{cov}\left(\Re \gamma_{h_{1}}^{(T)}\left(l_{1}, l_{2}\right), \Re \gamma_{h_{2}}^{(T)}\left(l_{1}, l_{2}\right)\right) \\
& =T \operatorname{cov}\left(\Im \gamma_{h_{1}}^{(T)}\left(l_{1}, l_{2}\right), \Im \gamma_{h_{2}}^{(T)}\left(l_{1}, l_{2}\right)\right) \\
& =\left\{\begin{array}{cl}
\frac{1}{4 \pi}\left(2 \pi+\int \frac{\mathcal{F}_{\omega}^{\left(l_{1}, l_{2}\right)} \mathcal{F}_{-\omega-\omega_{h}}^{\left(l_{2}, l_{1}\right)}}{\sqrt{\mathcal{F}_{\omega}^{\left(l_{1}, l_{1}\right)} \mathcal{F}_{-\omega}^{\left(l_{2}, l_{2}\right)} \mathcal{F}_{-\omega-\omega_{h}}^{\left(l_{2}, l_{2}\right)} \mathcal{F}_{\omega+\omega_{h}}^{\left(l_{1}, l_{1}\right)}}} d \omega\right. \\
\left.\quad+\iint \frac{\mathcal{F}_{\omega,-\omega-\omega_{h},-\omega^{\prime}}^{\left(l_{1} l_{2}, l_{1} l_{2}\right.}}{\sqrt{\mathcal{F}_{\omega}^{\left(l_{1}, l_{1}\right)} \mathcal{F}_{-\omega-\omega_{h}}^{\left(l_{2}, l_{2}\right)} \mathcal{F}_{-\omega^{\prime}}^{\left(l_{1}, l_{1}\right)} \mathcal{F}_{\omega^{\prime}+\omega_{h}}^{\left(l_{2}, l_{2}\right)}}} d \omega d \omega^{\prime}\right) & \text { if } h_{1}=h_{2}=h, \\
O\left(\frac{1}{T}\right) & \text { if } h_{1} \neq h_{2},
\end{array}\right.
\end{aligned}
$$

and $T \operatorname{cov}\left(\Re \gamma_{h_{1}}^{(T)}\left(l_{1}, l_{2}\right), \Im \gamma_{h_{2}}^{(T)}\left(l_{1}, l_{2}\right)\right)=O\left(T^{-1}\right)$ uniformly in $h_{1}, h_{2} \in \mathbb{Z}$. 


\section{B.6 Proofs under the alternative hypothesis of lo- cal stationarity}

Proof of Theorem 4.4.7. (i) In order to prove the first assertion of the theorem, introduce the bias-variance decomposition

$$
\begin{aligned}
\mathbb{E} & {\left[\left\|\hat{\mathcal{F}}_{\omega}^{(T)}-\mathbb{E}\left[\hat{\mathcal{F}}_{\omega}^{(T)}\right]+\mathbb{E}\left[\hat{\mathcal{F}}_{\omega}^{(T)}\right]-G_{\omega}\right\|_{2}^{2}\right] } \\
& =\mathbb{E}\left[\left\|\hat{\mathcal{F}}_{\omega}^{(T)}-\mathbb{E}\left[\hat{G}_{\omega}\right]\right\|_{2}^{2}\right]+\mathbb{E}\left[\left\|\mathbb{E}\left[\hat{\mathcal{F}}_{\omega}^{(T)}\right]-G_{\omega}\right\|_{2}^{2}\right] .
\end{aligned}
$$

The cross terms cancel because $\mathbb{E}\left[\left\langle\hat{\mathcal{F}}_{\omega}^{(T)}-\mathbb{E}\left[\hat{\mathcal{F}}_{\omega}^{(T)}\right], \mathbb{E}\left[\hat{\mathcal{F}}_{\omega}^{(T)}\right]-G_{\omega}\right\rangle_{H \otimes H}\right]$ and $\mathbb{E}\left[\hat{\mathcal{F}}_{\omega}^{(T)}\right.$ $\left.\mathbb{E}\left[\hat{\mathcal{F}}_{\omega}^{(T)}\right]\right]=O_{H}$. Now, by Lemma B.1.2,

$$
\operatorname{cum}\left(D_{\omega}^{(T)}, D_{-\omega}^{(T)}\right)=\frac{1}{T} \sum_{t=0}^{T-1} \mathcal{F}_{t / T, \omega}+R_{T, 2}=G_{\omega}^{(T)}+R_{T, 2},
$$

where $\left\|R_{T, 2}\right\|_{2}=O\left(T^{-1}\right)$. Convolution of the cumulant tensor with the smoothing kernel and subsequently a Taylor expansion gives

$$
\mathbb{E}\left[\hat{\mathcal{F}}_{\omega}^{(T)}\right]=\frac{2 \pi}{b T} \sum_{-=1}^{T} K_{b}\left(\omega-\omega_{j}\right) \operatorname{cum}\left(D_{\omega}^{(T)}, D_{-\omega}^{(T)}\right)=G_{\omega}+\epsilon_{b, T},
$$

where $\left\|\epsilon_{b, T}\right\|_{2}=O\left(b^{2}+(b T)\right)^{-1}$ and the interchange of summations is justified by Fubini's Theorem since $\sup _{\omega, u}\left\|f_{u, \omega}\right\|_{2}<\infty$ and $\sup _{\omega, u}\left\|\frac{\partial^{2}}{\partial \omega_{2}} f_{u, \omega}\right\|_{2}<\infty$,. Here, the error term $(b T)^{-1}$ follows from discretization of the window function (see e.g., Lemma P5.1 of Brillinger, 1981). Note that the integral approximation in time direction does not change the error term because of Lipschitz continuity in $u$. Thus, the second term in (4.60) satisfies

$$
\mathbb{E}\left[\left\|\mathbb{E} \hat{\mathcal{F}}_{\omega}^{(T)}-G_{\omega}\right\|_{2}^{2}\right]=O\left(b^{2}+\frac{1}{b T}\right)^{2} .
$$

To bound the first term of the right-hand side in (4.60), observe that, for $\omega \neq \omega^{\prime}$,

$$
\begin{aligned}
\operatorname{cum}\left(D_{\omega}^{(T)}, D_{\omega^{\prime}}^{(T)}\right) & =\frac{1}{2 \pi T} \sum_{t, t^{\prime}=1}^{T} \operatorname{cum}\left(X_{t}^{(T)}, X_{t^{\prime}}^{(T)}\right) e^{-\mathrm{i}\left(t \omega+t^{\prime} \omega^{\prime}\right)} \\
& =\frac{1}{2 \pi T} \sum_{t, t^{\prime}=1}^{T} \mathcal{C}_{t / T ; t^{\prime}-t}\left(\tau, \tau^{\prime}\right) e^{-\mathrm{i}\left(t^{\prime}-t\right) \omega^{\prime}-\mathrm{i} t\left(\omega+\omega^{\prime}\right)}+\epsilon_{t-t^{\prime}, T} \\
& =\frac{1}{2 \pi T} \sum_{t=1}^{T} \sum_{|h| \leqslant T-t} \operatorname{cum}\left(X_{t}^{(T)}, X_{t+h}^{(T)}\right) e^{-\mathrm{i}\left(h \omega^{\prime}\right)-\mathrm{i} t\left(\omega+\omega^{\prime}\right)}+R_{T, 2} \\
& =\frac{1}{2 \pi T} \sum_{t=1}^{T} \sum_{|h| \leqslant T-t} \mathcal{F}_{t / T, h} e^{-\mathrm{i}\left(h \omega^{\prime}\right)-\mathrm{i} t\left(\omega+\omega^{\prime}\right)}+R_{T, 2},
\end{aligned}
$$


where Lemma B.1.3 was applied to obtain the second equality sign and since further also

$$
\left\|\epsilon_{T}\right\|_{2}=\frac{1}{2 \pi T} \sum_{t_{1}=1}^{T} \frac{1+\left|t_{1}-t_{2}\right|}{T}\left\|\kappa_{2 ; t_{1}-t_{2}}\right\|_{2}=O\left(\frac{1}{T}\right)
$$

by (4.18). Decompose the corresponding local autocovariance operator of (4.61) as

$$
\frac{1}{2 \pi T}\left(\sum_{h=0}^{T-1} \sum_{t=1}^{T-h} \mathcal{C}_{t / T ; h} e^{-\mathrm{i}\left(h \omega^{\prime}\right)-\mathrm{i} t\left(\omega+\omega^{\prime}\right)}+\sum_{h=-T+1}^{-1} \sum_{t=1}^{T-|h|} \mathcal{C}_{t / T ; h} e^{-\mathrm{i}\left(h \omega^{\prime}\right)-\mathrm{i} t\left(\omega+\omega^{\prime}\right)}\right)+R_{T, 2}
$$

Under Assumption 4.4.6,

$$
\begin{gathered}
\left\|\frac { 1 } { 2 \pi T } \sum _ { h = 0 } ^ { T - 1 } \sum _ { t = 1 } ^ { T - h } \mathcal { C } _ { t / T , h } e ^ { - \mathrm { i } ( h \omega ^ { \prime } ) - \mathrm { i } t ( \omega + \omega ^ { \prime } ) } \left|\left\|_{2} \leqslant \frac{1}{2 \pi T} \sum_{h=0}^{T-1}\left|\sum_{t=1}^{T-h} e^{-\mathrm{i} t\left(\omega+\omega^{\prime}\right)}\right|\right\| \mathcal{C}_{t / T, h} \|_{2}\right.\right. \\
\leqslant \frac{1}{2 \pi T} \sum_{h=0}^{T-1}\left|\Delta_{T-h}^{\left(\omega+\omega^{\prime}\right)}\right|\left\|\mathcal{C}_{t / T, h}\right\|_{2} \leqslant \frac{C}{T} \sum_{h \in \mathbb{Z}}|h|\left\|\kappa_{2 ; h}\right\|_{2}=O\left(\frac{1}{T}\right)
\end{gathered}
$$

for some constant $C$. A similar derivation shows the same bound holds for the second term of (4.62). It can therefore be concluded that $\left\|\operatorname{cum}\left(D_{\omega}^{(T)}, D_{\lambda}^{(T)}\right)\right\| \|_{2}=O\left(T^{-1}\right)$ uniformly in $\omega \neq \omega^{\prime}, 0 \leqslant \omega, \omega^{\prime}<\pi$. Furthermore, Lemma B.1.2 and Minkowski's Inequality yield

$$
\begin{aligned}
\left\|\operatorname{cum}\left(D_{\omega}^{(T)}, D_{-\omega}^{(T)}, D_{\omega^{\prime}}^{(T)}, D_{-\omega^{\prime}}^{(T)}\right)\right\|_{2} & \leqslant \frac{1}{T}\left\|\frac{1}{T} \sum_{t=0}^{T-1} \mathcal{F}_{\frac{t}{T}, \omega,-\omega, \omega^{\prime}}\right\|_{2}+O\left(\frac{1}{T^{2}}\right) \\
& =\frac{1}{T}\left\|G_{\omega,-\omega, \omega^{\prime}}^{(T)}\right\|_{2}+O\left(\frac{1}{T^{2}}\right)=O\left(\frac{1}{T}\right) .
\end{aligned}
$$

The last equality follows since $\sup _{u, \omega}\left\|\mathcal{F}_{t / T, \omega,-\omega, \omega^{\prime}}\right\|_{2} \leqslant \sum_{h_{1}, h_{2}, h_{3} \in \mathbb{Z}}\left\|\kappa_{3 ; h_{1}, h_{2}, h_{3}}\right\|_{2}=$ $O(1)$ by Assumption 4.4.6. Therefore the product theorem for cumulant tensors (Theorem B.1.1) implies that

$$
\begin{aligned}
\operatorname{cov}\left(I_{\omega}^{(T)}, I_{\omega^{\prime}}^{(T)}\right)=\operatorname{cum} & \left(D_{\omega}^{(T)}, D_{-\omega}^{(T)}, D_{-\omega^{\prime}}^{(T)}, D_{\omega^{\prime}}^{(T)}\right) \\
& +S_{1324}\left(\operatorname{cum}\left(D_{\omega}^{(T)}, D_{-\omega^{\prime}}^{(T)}\right) \otimes \operatorname{cum}\left(D_{-\omega}^{(T)}, D_{\omega^{\prime}}^{(T)}\right)\right) \\
& +S_{1423}\left(\operatorname{cum}\left(D_{\omega}^{(T)}, D_{\omega^{\prime}}^{(T)}\right) \otimes \operatorname{cum}\left(D_{-\omega}^{(T)}, D_{-\omega^{\prime}}^{(T)}\right)\right)
\end{aligned}
$$

where $S_{i j k l}$ denotes the permutation operator on $\otimes_{i=1}^{4} L_{\mathbb{C}}^{2}([0,1])$ that permutes the components of a tensor according to the permutation $(1,2,3,4) \mapsto(i, j, k, l)$, that is, $S_{i j k l}\left(x_{1} \otimes \cdots \otimes x_{4}\right)=x_{i} \otimes \cdots \otimes x_{l}$. It is clear from (4.63) that $\left\|\operatorname{cov}\left(I_{\omega}^{(T)}, I_{\omega^{\prime}}^{(T)}\right)\right\|_{2}=$ $O\left(T^{-1}\right)$ for $\omega^{\prime} \neq \omega, 0 \leqslant \omega, \omega^{\prime}<\pi$, while for $\omega^{\prime}=\omega$ it follows that

$$
\begin{aligned}
\left\|\operatorname{cov}\left(I_{\omega}^{(T)}, I_{\omega}^{(T)}\right)\right\|_{2} \leqslant & \left\|S_{1324}\left(\operatorname{cum}\left(D_{\omega}^{(T)}, D_{-\omega}^{(T)}\right) \otimes \operatorname{cum}\left(D_{-\omega}^{(T)}, D_{\omega}^{(T)}\right)\right)\right\|_{2} \\
& +\left\|S_{1423}\left(\operatorname{cum}\left(D_{\omega}^{(T)}, D_{\omega}^{(T)}\right) \otimes \operatorname{cum}\left(D_{-\omega}^{(T)}, D_{-\omega}^{(T)}\right)\right)\right\|_{2}+R_{T, 2}=O(1) .
\end{aligned}
$$


Furthermore,

$$
\begin{aligned}
\operatorname{cov}\left(\hat{\mathcal{F}}_{\omega}^{(T)}, \hat{\mathcal{F}}_{\omega}^{(T)}\right)= & \left(\frac{2 \pi}{T}\right)^{2} \sum_{j, j^{\prime}=1}^{T} K_{b}\left(\omega-\omega_{j}\right) K_{b}\left(\omega-\omega_{j^{\prime}}\right) \\
& \times \frac{1}{T^{2}}\left(\sum_{t, t^{\prime}=0}^{T-1} \mathcal{F}_{t / T, \omega_{j}} \otimes \mathcal{F}_{t / T,-\omega_{j}} e^{-\mathrm{i}\left(t-t^{\prime}\right)\left(\omega_{j}-\omega_{j^{\prime}}\right)}\right. \\
& \left.+\sum_{t, t^{\prime}=0}^{T-1} \mathcal{F}_{t / T, \omega_{j}} \otimes \mathcal{F}_{t / T,-\omega_{j}} e^{-\mathrm{i}\left(t-t^{\prime}\right)\left(\omega_{j}+\omega_{j^{\prime}}\right)}\right)+R_{T, 2} .
\end{aligned}
$$

Hence,

$$
\begin{aligned}
& \left\|\operatorname{cov}\left(\hat{\mathcal{F}}_{\omega}^{(T)}, \hat{\mathcal{F}}_{\omega}^{(T)}\right)\right\| \|_{2} \leqslant \\
& \quad \sup _{u, \omega}\left\|\mathcal{F}_{u, \omega}\right\|_{2}^{2}\left(\frac{2 \pi}{T}\right)^{2} \sum_{j, j^{\prime}=1}^{T} K_{b}\left(\omega-\omega_{j}\right) K_{b}\left(\omega-\omega_{j^{\prime}}\right) \frac{\left|\Delta_{T}^{\left(\omega_{j}-\omega_{j^{\prime}}\right)}\right|^{2}}{T^{2}} \\
& +\sup _{u, \omega}\left\|\mathcal{F}_{u, \omega}\right\|_{2}^{2}\left(\frac{2 \pi}{T}\right)^{2} \sum_{j, j^{\prime}=1}^{T} K_{b}\left(\omega-\omega_{j}\right) K_{b}\left(\omega-\omega_{j^{\prime}}\right) \frac{\left|\Delta_{T}^{\left(\omega_{j}+\omega_{j^{\prime}}\right)}\right|^{2}}{T^{2}}+O\left(\frac{1}{T}\right) \\
& =O\left(\frac{1}{b T}\right) .
\end{aligned}
$$

Together with the equivalence of the Hilbert-Schmidt norm of the operator and the $L_{2}-$ norm of its kernel, the above implies then that the second term of (4.60) satisfies

$$
\mathbb{E}\left[\left\|\hat{\mathcal{F}}_{\omega}^{(T)}-\mathbb{E} \hat{\mathcal{F}}_{\omega}^{(T)}\right\|_{2}^{2}\right]=\int_{[0,1]^{2}} \operatorname{var}\left(\hat{f}_{\omega}^{(T)}\left(\tau, \tau^{\prime}\right)\right) d \tau d \tau^{\prime}=O\left(\frac{1}{b T}\right)
$$

uniformly in $\omega \in[-\pi, \pi]$. This establishes (i).

(ii) The second part of the proof proceeds along similar lines as Paparoditis (2009). An application of Minkowski's inequality yields

$$
\begin{aligned}
\left\|\hat{\mathcal{F}}_{\omega}^{(T)}-G_{\omega}\right\|_{2} \leqslant \| & \frac{2 \pi}{T} \sum_{j=1}^{T} K_{b}\left(\omega_{j^{\prime}}-\omega_{j}\right)\left[\operatorname{cum}\left(D_{\omega_{j}}^{(T}, D_{-\omega_{j}}^{(T)}\right)-\frac{1}{T} \sum_{t=1} \mathcal{F}_{t / T, \omega_{j}}\right] \|_{2} \\
& +\left\|\frac{2 \pi}{T} \sum_{j=1}^{T} K_{b}\left(\omega_{j^{\prime}}-\omega_{j}\right) \frac{1}{T} \sum_{t=1}\left[f_{t / T, \omega_{j}}-\mathcal{F}_{t / T, \omega}\right]\right\|_{2} \\
& +\left\|\left(\frac{2 \pi}{T} \sum_{j=1}^{T} K_{b}\left(\omega_{j^{\prime}}-\omega_{j}\right)-1\right) \frac{1}{T} \sum_{t=1} \mathcal{F}_{t / T, \omega}\right\|_{2} .
\end{aligned}
$$

Markov's inequality together with (i), which is not affected by the discretization of the integral, imply the first term tends to zero. Since the spectral operator is Lipschitz continuous in $\omega$, the second term is bounded by

$$
\left|1-\int_{-\pi}^{\pi} K_{b}\left(\omega_{k}-\omega^{\prime}\right) d \omega^{\prime}\right||b|=O\left(1+\frac{1}{b T}\right) O(b)=O(b) .
$$

Finally, the third term is seen to be of order $O\left((b T)^{-1}\right)$. 
Proof of Theorem 4.4.10. By Lemma B.1.2, the expectation of $\gamma_{h}^{(T)}\left(l, l^{\prime}\right)$ satisfies

$$
\mathbb{E}\left[\gamma_{h}^{(T)}\left(l, l^{\prime}\right)\right]=\frac{1}{T} \sum_{j=1}^{T}\left[\frac{1}{T} \sum_{t=0}^{T-1} \frac{\mathcal{F}_{t / T, \omega_{j}}^{\left(l, l^{\prime}\right)}}{\left(G_{\omega_{j}}^{(l, l)} G_{\omega_{j+h}}^{\left(l^{\prime}, l^{\prime}\right)}\right){ }^{1 / 2}} e^{-\mathrm{i} t \omega_{h}}+O\left(\frac{1}{T}\right)\right]=O\left(\frac{1}{h^{2}}+\frac{1}{T}\right)
$$

for all $h=1, \ldots, T-1$. Using the Lipschitz continuity of the spectra, expression (4.22) follows. For the covariance structure, note once more that

$\Re \gamma_{h}^{(T)}\left(l, l^{\prime}\right)=\frac{1}{2}\left(\gamma_{h}^{(T)}\left(l, l^{\prime}\right)+\overline{\gamma_{h}^{(T)}\left(l, l^{\prime}\right)}\right) \quad$ and $\quad \Im \gamma_{h}^{(T)}\left(l, l^{\prime}\right)=\frac{1}{2 \mathrm{i}}\left(\gamma_{h}^{(T)}\left(l, l^{\prime}\right)-\overline{\gamma_{h}^{(T)}\left(l, l^{\prime}\right)}\right)$.

Under the alternative, these are in fact correlated and four separate cases will have to be considered:

(i) $\operatorname{cov}\left(\Re \gamma_{h_{1}}^{\left(l_{1}, l_{2}\right)}, \Re \gamma_{h_{2}}^{\left(l_{3}, l_{4}\right)}\right)=\frac{1}{4}\left[\operatorname{cov}\left(\gamma_{h_{1}}^{\left(l_{1}, l_{2}\right)}, \gamma_{h_{2}}^{\left(l_{3}, l_{4}\right)}\right)+\operatorname{cov}\left(\gamma_{h_{1}}^{\left(l_{1}, l_{2}\right)}, \overline{\gamma_{h_{2}}^{\left(l_{3}, l_{4}\right)}}\right)\right.$

$$
\left.+\operatorname{cov}\left(\overline{\gamma_{h_{1}}^{\left(l_{1}, l_{2}\right)}}, \gamma_{h_{2}}^{\left(l_{3}, l_{4}\right)}\right)+\operatorname{cov}\left(\overline{\gamma_{h_{1}}^{\left(l_{1}, l_{2}\right)}}, \overline{\gamma_{h_{2}}^{\left(l_{3}, l_{4}\right)}}\right)\right]
$$

(ii) $\operatorname{cov}\left(\Re \gamma_{h_{1}}^{\left(l_{1}, l_{2}\right)}, \Im \gamma_{h_{2}}^{\left(l_{3}, l_{4}\right)}\right)=\frac{1}{4 \overline{\mathrm{i}}}\left[\operatorname{cov}\left(\gamma_{h_{1}}^{\left(l_{1}, l_{2}\right)}, \gamma_{h_{2}}^{\left(l_{3}, l_{4}\right)}\right)-\operatorname{cov}\left(\gamma_{h_{1}}^{\left(l_{1}, l_{2}\right)}, \overline{\gamma_{h_{2}}^{\left(l_{3}, l_{4}\right)}}\right)\right.$

$$
\left.+\operatorname{cov}\left(\overline{\gamma_{h_{1}}^{\left(l_{1}, l_{2}\right)}}, \gamma_{h_{2}}^{\left(l_{3}, l_{4}\right)}\right)-\operatorname{cov}\left(\overline{\gamma_{h_{1}}^{\left(l_{1}, l_{2}\right)}}, \overline{\gamma_{h_{2}}^{\left(l_{3}, l_{4}\right)}}\right)\right]
$$

(iii) $\operatorname{cov}\left(\Im \gamma_{h_{1}}^{\left(l_{1}, l_{2}\right)}, \Re \gamma_{h_{2}}^{\left(l_{3}, l_{4}\right)}\right)=\frac{1}{4 \overline{\mathrm{i}}}\left[\operatorname{cov}\left(\gamma_{h_{1}}^{\left(l_{1}, l_{2}\right)}, \gamma_{h_{2}}^{\left(l_{3}, l_{4}\right)}\right)+\operatorname{cov}\left(\gamma_{h_{1}}^{\left(l_{1}, l_{2}\right)}, \overline{\gamma_{h_{2}}^{\left(l_{3}, l_{4}\right)}}\right)\right.$

$$
\left.-\operatorname{cov}\left(\overline{\gamma_{h_{1}}^{\left(l_{1}, l_{2}\right)}}, \gamma_{h_{2}}^{\left(l_{3}, l_{4}\right)}\right)-\operatorname{cov}\left(\overline{\gamma_{h_{1}}^{\left(l_{1}, l_{2}\right)}}, \overline{\gamma_{h_{2}}^{\left(l_{3}, l_{4}\right)}}\right)\right]
$$

(iv) $\operatorname{cov}\left(\Im \gamma_{h_{1}}^{\left(l_{1}, l_{2}\right)}, \Im \gamma_{h_{2}}^{\left(l_{3}, l_{4}\right)}\right)=\frac{1}{4}\left[\operatorname{cov}\left(\gamma_{h_{1}}^{\left(l_{1}, l_{2}\right)}, \gamma_{h_{2}}^{\left(l_{3}, l_{4}\right)}\right)-\operatorname{cov}\left(\gamma_{h_{1}}^{\left(l_{1}, l_{2}\right)}, \overline{\gamma_{h_{2}}^{\left(l_{3}, l_{4}\right)}}\right)\right.$

$$
\left.-\operatorname{cov}\left(\overline{\gamma_{h_{1}}^{\left(l_{1}, l_{2}\right)}}, \gamma_{h_{2}}^{\left(l_{3}, l_{4}\right)}\right)+\operatorname{cov}\left(\overline{\gamma_{h_{1}}^{\left(l_{1}, l_{2}\right)}}, \overline{\gamma_{h_{2}}^{\left(l_{3}, l_{4}\right)}}\right)\right] .
$$

To find the expressions for the covariance structure of $\sqrt{T} \gamma_{h}^{(T)}$ and its complex conjugate, use (4.57) and Lemma B.1.2 to write

$$
\begin{aligned}
\operatorname{cov}\left(D_{\omega_{j_{1}}}^{\left(l_{1}\right)} D_{-\omega_{j_{1}+h_{1}}}^{\left(l_{2}\right)}, D_{\omega_{j_{2}}}^{\left(l_{3}\right)} D_{-\omega_{j_{2}+h_{2}}}^{\left(l_{4}\right)}\right) \\
=\frac{2 \pi}{T} \tilde{\mathcal{F}}_{\left(-h_{1}+h_{2} ; \omega_{j_{1}},-\omega_{j_{1}+h_{1}},-\omega_{j_{2}}\right)}^{\left(l_{1} l_{2}, l_{3} l_{l_{1}}\right)}+O\left(\frac{1}{T^{2}}\right) \\
\quad+\left[\tilde{\mathcal{F}}_{\left(j_{1}-j_{2} ; \omega_{j_{1}}\right)}^{\left(l_{1}, l_{3}\right)}+O\left(\frac{1}{T}\right)\right]\left[\tilde{\mathcal{F}}_{\left(-j_{1}-h_{1}+j_{2}+h_{2} ;-\omega_{\left.j_{1}+h_{1}\right)}\right.}^{\left(l_{2}, l_{4}\right)}+O\left(\frac{1}{T}\right)\right] \\
+\left[\tilde{\mathcal{F}}_{\left(j_{1}+j_{2}+h_{2} ; \omega_{j_{1}}\right)}^{\left(l_{1}, l_{4}\right)}+O\left(\frac{1}{T}\right)\right]\left[\tilde{\mathcal{F}}_{\left(-j_{1}-h_{1}-j_{2},-\omega_{\left.j_{1}+h_{1}\right)}\right.}^{\left(l_{2}, l_{3}\right)}+O\left(\frac{1}{T}\right)\right] .
\end{aligned}
$$


Thus,

$$
\begin{aligned}
& \operatorname{cov}\left(\sqrt{T} \gamma_{h_{1}}^{(T)}\left(l_{1}, l_{2}\right), \sqrt{T} \gamma_{h_{2}}^{(T)}\left(l_{3}, l_{4}\right)\right) \\
& =\frac{1}{T} \sum_{j_{1}, j_{2}=1}^{T}\left\{\frac{2 \pi}{T} \frac{\tilde{\mathcal{F}}_{\left(-h_{1}+h_{2} ; \omega_{j_{1}},-\omega_{j_{1}+h_{1}},-\omega_{j_{2}}\right)}^{\left(l_{1} l_{2}, l_{2} l_{4}\right)}}{\left(G_{\omega_{j_{1}}}^{\left(l_{1}, l_{1}\right)} G_{-\omega_{j_{1}+h_{1}}}^{\left(l_{2}, l_{2}\right)} G_{-\omega_{j_{2}}}^{\left(l_{3}, l_{3}\right)} G_{\omega_{j_{2}}+h_{2}}^{\left(l_{4}, l_{4}\right)}\right)^{1 / 2}}+\frac{\tilde{\mathcal{F}}_{\left(j_{1}-j_{2} ; \omega_{j_{1}}\right)}^{\left(l_{1}, l_{3}\right)} \tilde{\mathcal{F}}_{\left(-j_{1}-h_{1}+j_{2}+h_{2} ;-\omega_{j_{1}+h_{1}}\right)}^{\left(l_{2}, l_{4}\right)}}{\left(G_{\omega_{j_{1}}}^{\left(l_{1}, l_{1}\right)} G_{-\omega_{j_{2}}}^{\left(l_{3}, l_{3}\right)} G_{-\omega_{j_{1}+h_{1}}}^{\left(l_{2}, l_{2}\right)} G_{\omega_{j_{2}}+h_{2}}^{\left(l_{4}, l_{4}\right)}\right)^{1 / 2}}\right. \\
& +\frac{\tilde{\mathcal{F}}_{\left(j_{1}+j_{2}+h_{2} ; \omega_{j_{1}}\right)}^{\left(l_{1}, l_{4}\right)} \tilde{\mathcal{F}}_{\left(-j_{1}-h_{1}-j_{2},-\omega_{j_{1}+h_{1}}\right)}^{\left(l_{2}, l_{3}\right)}}{\left(G_{\omega_{j_{1}}}^{\left(l_{1}, l_{1}\right)} G_{\omega_{j_{2}+h_{2}}}^{\left(l_{4}, l_{4}\right)} G_{-\omega_{j_{1}+h_{1}}}^{\left(l_{2}, l_{2}\right)} G_{-\omega_{j_{2}}}^{\left(l_{3}, l_{3}\right)}\right)^{1 / 2}} \\
& +O\left(\frac { 1 } { T } \left[\frac{\tilde{\mathcal{F}}_{\left(j_{1}-j_{2} ; \omega_{j_{1}}\right)}^{\left(l_{1}, l_{3}\right)}}{\left(G_{\omega_{j_{1}}}^{\left(l_{1}, l_{1}\right)} G_{-\omega_{j_{2}}}^{\left(l_{3}, l_{3}\right)}\right)^{1 / 2}}+\frac{\tilde{\mathcal{F}}_{\left(-j_{1}-h_{1}+j_{2}+h_{2} ;-\omega_{j_{1}+h_{1}}\right)}^{\left(l_{2}, l_{4}\right)}}{\left(G_{-\omega_{j_{1}+h_{1}}}^{\left(l_{2}, l_{2}\right)} G_{\omega_{j_{2}}+h_{2}}^{\left(l_{4}, l_{4}\right)}\right)^{1 / 2}}\right.\right. \\
& \left.\left.\left.+\frac{\tilde{\mathcal{F}}_{\left(j_{1}+j_{2}+h_{2} ; \omega_{j_{1}}\right)}^{\left(l_{1}, l_{4}\right)}}{\left(G_{\omega_{j_{1}}}^{\left(l_{1}, l_{1}\right)} G_{\omega_{j_{2}}+h_{2}}^{\left(l_{4}, l_{4}\right)}\right)^{1 / 2}}+\frac{\tilde{\mathcal{F}}_{\left(-j_{1}-h_{1}-j_{2},-\omega_{j_{1}+h_{1}}\right)}^{\left(l_{2}, l_{3}\right)}}{\left(G_{-\omega_{j_{1}+h_{1}}}^{\left(l_{2}, l_{2}\right)} G_{-\omega_{j_{2}}}^{\left(l_{3}, l_{3}\right)}\right)^{1 / 2}}\right]+\frac{1}{T^{2}}\right)\right\} .
\end{aligned}
$$

By Corollary B.1.2 (ii), this equals

$$
\begin{aligned}
\Sigma_{h_{1}, h_{2}}^{(T)}\left(\boldsymbol{l}_{4}\right)= & T \operatorname{cov}\left(\gamma_{h_{1}}^{(T)}\left(l_{1}, l_{2}\right), \gamma_{h_{2}}^{(T)}\left(l_{3}, l_{4}\right)\right) \\
=\frac{1}{T} & \sum_{j_{1}, j_{2}=1}^{T} \mathcal{G}_{j_{1}, j_{2}}^{\left(l_{1}, l_{2}, l_{3}, l_{4}\right)}\left(\tilde{\mathcal{F}}_{\left(j_{1}-j_{2} ; \omega_{j_{1}}\right)}^{\left(l_{1}, l_{3}\right)} \tilde{\mathcal{F}}_{\left(-j_{1}-h_{1}+j_{2}+h_{2} ;-\omega_{j_{1}+h_{1}}\right)}^{\left(l_{2}, l_{4}\right)}\right. \\
& \left.+\tilde{\mathcal{F}}_{\left(j_{1}+j_{2}+h_{2} ; \omega_{j_{1}}\right)}^{\left(l_{1}, l_{4}\right)} \tilde{\mathcal{F}}_{\left(-j_{1}-h_{1}-j_{2},-\omega_{\left.j_{1}+h_{1}\right)}\right.}^{\left(l_{2}, l_{3}\right)}+\frac{2 \pi}{T} \tilde{\mathcal{F}}_{\left(-h_{1}+h_{2} ; \omega_{j_{1}},-\omega_{j_{1}+h_{1}},-\omega_{j_{2}}\right)}^{\left(l_{1} l_{2}, l_{3} l_{4}\right)}\right) \\
& +O\left(\frac{1}{T}\right)
\end{aligned}
$$

where $\mathcal{G}_{j_{1}, j_{2}}^{\left(l_{1}, l_{2}, l_{3}, l_{4}\right)}=\left(G_{\omega_{j_{1}}}^{\left(l_{1}, l_{1}\right)} G_{\omega_{j_{1}+h_{1}}}^{\left(l_{2}, l_{2}\right)} G_{\omega_{j_{2}}}^{\left(l_{3}, l_{3}\right)} G_{\omega_{j_{2}+h_{2}}}^{\left(l_{4}, l_{4}\right)}\right)^{-1 / 2}$. Similarly,

$$
\begin{aligned}
& \dot{\Sigma}_{h_{1}, h_{2}}^{(T)}\left(\boldsymbol{l}_{4}\right)=T \operatorname{cov}\left(\gamma_{h_{1}}^{(T)}\left(l_{1}, l_{2}\right), \overline{\gamma_{h_{2}}^{(T)}\left(l_{3}, l_{4}\right)}\right) \\
& =\frac{1}{T} \sum_{j_{1}, j_{2}=1}^{T} \mathcal{G}_{j_{1}, j_{2}}^{\left(l_{1}, l_{2}, l_{3}, l_{4}\right)}\left(\tilde{\mathcal{F}}_{\left(j_{1}+j_{2} ; \omega_{j_{1}}\right)}^{\left(l_{1}, l_{3}\right)} \tilde{\mathcal{F}}_{\left(-j_{1}-h_{1}-j_{2}-h_{2} ;-\omega_{j_{1}+h_{1}}\right)}^{\left(l_{2}, l_{4}\right)}\right. \\
& \left.+\tilde{\mathcal{F}}_{\left(j_{1}-j_{2}-h_{2} ; \omega_{j_{1}}\right)}^{\left(l_{1}, l_{4}\right)} \tilde{\mathcal{F}}_{\left(-j_{1}-h_{1}+j_{2} ;-\omega_{j_{1}+h_{1}}\right)}^{\left(l_{2}, l_{3}\right)}+\frac{2 \pi}{T} \tilde{\mathcal{F}}_{\left(-h_{1}-h_{2} ; \omega_{j_{1}},-\omega_{j_{1}+h_{1}}, \omega_{j_{2}}\right)}^{\left(l_{1} l_{2}, l_{3} l_{4}\right)}\right) \\
& +O\left(\frac{1}{T}\right) \\
& \bar{\Sigma}_{h_{1}, h_{2}}^{(T)}\left(\boldsymbol{l}_{4}\right)=T \operatorname{cov}\left(\overline{\gamma_{h_{1}}^{(T)}\left(l_{1}, l_{2}\right)}, \overline{\gamma_{h_{2}}^{(T)}\left(l_{3}, l_{4}\right)}\right) \\
& =\frac{1}{T} \sum_{j_{1}, j_{2}=1}^{T} \mathcal{G}_{j_{1}, j_{2}}^{\left(l_{1}, l_{2}, l_{3}, l_{4}\right)}\left(\tilde{\mathcal{F}}_{\left(-j_{1}+j_{2} ;-\omega_{j_{1}}\right)}^{\left(l_{1}, l_{3}\right)} \tilde{\mathcal{F}}_{\left(j_{1}+h_{1}-j_{2}-h_{2} ; \omega_{j_{1}+h_{1}}\right)}^{\left(l_{2}, l_{4}\right)}\right. \\
& \left.+\tilde{\mathcal{F}}_{\left(-j_{1}-j_{2}-h_{2} ;-\omega_{j_{1}}\right)}^{\left(l_{1}, l_{4}\right)} \tilde{\mathcal{F}}_{\left(j_{1}+h_{1}+j_{2} ; \omega_{j_{1}+h_{1}}\right)}^{\left(l_{2}, l_{3}\right)}+\frac{2 \pi}{T} \tilde{\mathcal{F}}_{\left(h_{1}-h_{2} ;-\omega_{j_{1}}, \omega_{j_{1}+h_{1}}, \omega_{j_{2}}\right)}^{\left(l_{1} l_{2}, l_{3} l_{4}\right)}\right) \\
& +O\left(\frac{1}{T}\right)
\end{aligned}
$$


and

$$
\begin{aligned}
\grave{\Sigma}_{h_{1}, h_{2}}^{(T)}\left(\boldsymbol{l}_{4}\right)= & \left.T \operatorname{cov} \overline{\left(\gamma_{h_{1}}^{(T)}\left(l_{1}, l_{2}\right)\right.}, \gamma_{h_{2}}^{(T)}\left(l_{3}, l_{4}\right)\right) \\
=\frac{1}{T} & \sum_{j_{1}, j_{2}=1}^{T}\left(\mathcal{G}_{j_{1}, j_{2}}^{\left(l_{1}, l_{2}, l_{3}, l_{4}\right)} \tilde{\mathcal{F}}_{\left(-j_{1}-j_{2} ;-\omega_{j_{1}}\right)}^{\left(l_{1}, l_{3}\right)} \tilde{\mathcal{F}}_{\left(j_{1}+h_{1}+j_{2}+h_{2} ; \omega_{j_{1}+h_{1}}\right)}^{\left(l_{2}, l_{4}\right)}\right. \\
& \left.+\tilde{\mathcal{F}}_{\left(-j_{1}+j_{2}+h_{2} ;-\omega_{j_{1}}\right)}^{\left(l_{1}, l_{4}\right)} \tilde{\mathcal{F}}_{\left(j_{1}+h_{1}-j_{2} ; \omega_{\left.j_{1}+h_{1}\right)}\right.}^{\left(l_{2}, l_{3}\right)}+\frac{2 \pi}{T} \tilde{\mathcal{F}}_{\left(h_{1}+h_{2} ;-\omega_{j_{1}}, \omega_{j_{1}+h_{1}},-\omega_{j_{2}}\right)}^{\left(l_{1} l_{2}, l_{3} l_{4}\right)}\right) \\
& +O\left(\frac{1}{T}\right) .
\end{aligned}
$$

This completes the proof.

Similarly, we find for the covariance structure of Theorem B.4.4:

$$
\begin{aligned}
& \Upsilon_{h_{1}, h_{2}}\left(\psi_{l_{1} l_{1}^{\prime} l_{2} l_{2}^{\prime}}\right)=\lim _{T \rightarrow \infty} \frac{1}{T} \sum_{j_{1}, j_{2}=1}^{T}\left(\left\langle\tilde{\mathcal{F}}_{j_{1}-j_{2} ; \omega_{j_{1}}}\left(\psi_{l_{2}}\right), \psi_{l_{1}}\right\rangle\left\langle\tilde{\mathcal{F}}_{-j_{1}-h_{1}+j_{2}+h_{2} ;-\omega_{j_{1}+h_{1}}}\left(\psi_{l_{2}^{\prime}}\right), \psi_{l_{1}^{\prime}}\right\rangle\right. \\
& +\left\langle\tilde{\mathcal{F}}_{j_{1}+j_{2}+h_{2} ; \omega_{j_{1}}}\left(\psi_{l_{2}^{\prime}}\right), \psi_{l_{1}}\right\rangle\left\langle\tilde{\mathcal{F}}_{-j_{1}-h_{1}-j_{2},-\omega_{j_{1}+h_{1}}}\left(\psi_{l_{2}}\right), \psi_{l_{1}^{\prime}}\right\rangle \\
& \left.+\frac{2 \pi}{T}\left\langle\tilde{\mathcal{F}}_{\left(-h_{1}+h_{2} ; \omega_{j_{1}},-\omega_{j_{1}+h_{1}},-\omega_{j_{2}}\right)}\left(\psi_{l_{2}} l_{2}^{\prime}\right), \psi_{l_{1}, l_{1}^{\prime}}\right\rangle\right), \\
& \dot{\Upsilon}_{h_{1}, h_{2}}\left(\psi_{l_{1} l_{1}^{\prime} l_{2} l_{2}^{\prime}}\right)=\lim _{T \rightarrow \infty} \frac{1}{T} \sum_{j_{1}, j_{2}=1}^{T}\left(\left\langle\tilde{\mathcal{F}}_{j_{1}+j_{2} ; \omega_{j_{1}}}\left(\psi_{l_{2}}\right), \psi_{l_{1}}\right\rangle\left\langle\tilde{\mathcal{F}}_{-j_{1}-h_{1}-j_{2}-h_{2} ;-\omega_{j_{1}+h_{1}}}\left(\psi_{l_{2}^{\prime}}\right), \psi_{l_{1}^{\prime}}\right\rangle\right. \\
& +\left\langle\tilde{\mathcal{F}}_{j_{1}-j_{2}-h_{2} ; \omega_{j_{1}}}\left(\psi_{l_{2}^{\prime}}\right), \psi_{l_{1}}\right\rangle\left\langle\tilde{\mathcal{F}}_{-j_{1}-h_{1}+j_{2} ;-\omega_{j_{1}+h_{1}}}\left(\psi_{l_{2}}\right), \psi_{l_{1}^{\prime}}\right\rangle \\
& \left.+\frac{2 \pi}{T}\left\langle\tilde{\mathcal{F}}_{\left(-h_{1}-h_{2} ; \omega_{j_{1}},-\omega_{j_{1}+h_{1}}, \omega_{j_{2}}\right)}\left(\psi_{l_{2} l_{2}^{\prime}}\right), \psi_{l_{1} l_{1}^{\prime}}\right\rangle\right), \\
& \bar{\Upsilon}_{h_{1}, h_{2}}\left(\psi_{l_{1} l_{1}^{\prime} l_{2} l_{2}^{\prime}}\right)=\lim _{T \rightarrow \infty} \frac{1}{T} \sum_{j_{1}, j_{2}=1}^{T}\left(\left\langle\tilde{\mathcal{F}}_{-j_{1}+j_{2} ;-\omega_{j_{1}}}\left(\psi_{l_{2}}\right), \psi_{l_{1}}\right\rangle\left\langle\tilde{\mathcal{F}}_{j_{1}+h_{1}-j_{2}-h_{2} ; \omega_{j_{1}+h_{1}}}\left(\psi_{l_{2}^{\prime}}\right), \psi_{l_{1}^{\prime}}\right\rangle\right. \\
& +\left\langle\tilde{\mathcal{F}}_{-j_{1}-j_{2}-h_{2} ;-\omega_{j_{1}}}\left(\psi_{l_{2}^{\prime}}\right), \psi_{l_{1}}\right\rangle\left\langle\tilde{\mathcal{F}}_{j_{1}+h_{1}+j_{2} ; \omega_{j_{1}+h_{1}}}\left(\psi_{l_{2}}\right), \psi_{l_{1}^{\prime}}\right\rangle \\
& \left.+\frac{2 \pi}{T}\left\langle\tilde{\mathcal{F}}_{\left(h_{1}-h_{2} ;-\omega_{j_{1}}, \omega_{j_{1}+h_{1}}, \omega_{j_{2}}\right)}\left(\psi_{l_{2} l_{2}^{\prime}}\right), \psi_{l_{1} l_{1}^{\prime}}\right\rangle\right)
\end{aligned}
$$


and

$$
\begin{aligned}
\grave{\Upsilon}_{h_{1}, h_{2}}\left(\psi_{l_{1} l_{1}^{\prime} l_{2} l_{2}^{\prime}}\right)=\lim _{T \rightarrow \infty} \frac{1}{T} \sum_{j_{1}, j_{2}=1}^{T} & \left(\left\langle\tilde{\mathcal{F}}_{-j_{1}-j_{2} ;-\omega_{j_{1}}}\left(\psi_{l_{2}}\right), \psi_{l_{1}}\right\rangle\left\langle\tilde{\mathcal{F}}_{j_{1}+h_{1}+j_{2}+h_{2} ; \omega_{j_{1}+h_{1}}}\left(\psi_{l_{2}^{\prime}}\right), \psi_{l_{1}^{\prime}}\right\rangle\right. \\
+ & \left\langle\tilde{\mathcal{F}}_{-j_{1}+j_{2}+h_{2} ;-\omega_{j_{1}}}\left(\psi_{l_{2}^{\prime}}\right), \psi_{l_{1}}\right\rangle\left\langle\tilde{\mathcal{F}}_{j_{1}+h_{1}-j_{2} ; \omega_{j_{1}+h_{1}}}\left(\psi_{l_{2}}\right), \psi_{l_{1}^{\prime}}\right\rangle \\
+ & \left.\frac{2 \pi}{T}\left\langle\tilde{\mathcal{F}}_{\left(h_{1}+h_{2} ;-\omega_{j_{1}}, \omega_{j_{1}+h_{1}},-\omega_{j_{2}}\right)}\left(\psi_{l_{2}} l_{2}^{\prime}\right), \psi_{l_{1} l_{1}^{\prime}}\right\rangle\right)
\end{aligned}
$$





\section{Chapter 5}

\section{Conclusion}

This chapter concludes this thesis. Because each chapter contains its own conclusion, I will refrain from going into details. Rather, this chapter focuses on shortly providing an overview of each, where limitations and possible extensions for future research are highlighted. This is then followed by an overall conclusion.

This thesis dealt with the analysis of stochastic processes with time-varying spectral characteristics. Each chapter contributed to this topic in a different way. Chapter 2 focused on the development of an algorithm for the nonparametric estimation of time-varying spectra that allows to data-adaptively determine the optimal shape of the smoothing kernel. Chapter 3 was concerned with the introduction of a theoretical framework enabling statistical inference for nonstationary functional time series, i.e., time series that take values in an infinite-dimensional separable Hilbert space. Chapter 4 covered the problem of identification of nonstationary functional time series by means of a frequency domain-based test statistic. This thesis therefore tackled both a long-time open practical problem in the analysis of time-varying spectra, as well as the gap in theory for the analysis of time series on infinite-dimensional separable Hilbert spaces that are characterized by changing second-order characteristics.

A data-adaptive approach for the estimation of time-varying spectra was introduced in Chapter 2. The aim of this chapter has been to develop a method that circumvents the explicit specification of the smoothing bandwidths in time and frequency direction, parameters for which no guidelines are available in practice and for which the optimal theoretical values are often not optimal in finite samples. To overcome this sensitivity, the idea behind the approach has been to adjust the degree of frequency and time resolution to the data at hand. This is achieved by constructing the smoothing kernel - viewed as a bivariate function in the time-frequency plane - iteratively. In each step, the adaptive smoothing kernel is applied to a very raw estimator, the pre-periodogram, from which estimates of the local spectral variates are subsequently obtained. The algorithm starts with a small initial search neighborhood around the point of interest. The new adaptive smoothing kernel is then constructed based on those points within this neighborhood for which the condition of 'homogeneity' is not violated. This is done for all points in the time-frequency plane and new estimates of the local spectral variates are obtained by applying the 
updated resulting smoothing kernels to the corresponding pre-periodogram variates. The search neighborhoods are then extended and the same is repeated until the maximum bandwidths are reached.

The method has a couple of interesting features. Firstly, the smoothing kernels are not constructed as the product of a kernel in time and frequency direction but really as a bivariate function in the plane. The advantage is that the smoothing kernel can be of any shape and therefore permits full flexibility. This property enables not only for easy adjustment to smooth changes in the spectrum but at the same time makes it possible to automatically adjust for structural breaks in the spectrum. Moreover, the method is tailored to control for the volatile behavior of the pre-periodogram over iterations. This volatile behavior and the resulting artifacts are usually a reason why the pre-periodogram, and other spectrogram-like estimators, are not often used in practice. It is however the 'rawness' of these type of estimators that enables the flexibility in adjusting the degree of frequency and time resolution. Many applications could benefit from such flexibility and I therefore believe a more thorough understanding of the pre-periodogram can be of general importance.

Of course, the procedure has its limitations. There are a few initial parameters that need to be specified. However, simulation studies show the performance is not sensitive to these parameters. Theoretical guidelines would however be useful in understanding how to fine-tune the method. The main drawback would be that the full adaptability of the algorithm comes at the cost of a long running time. It is however expected that future technology and an implementation by means of the graphical processing unit can make this issue negligible. This is left for future research.

In contrast to Chapter 2, in which a practical problem was addressed, Chapter 3 and Chapter 4 focused on the development of theory and methodology for the analysis of nonstationary stochastic processes taking values in the function space. Technological advances in data storage techniques have led to a surge in demand for methodology that allows to extract information and to draw inferences from data that can be viewed as being sampled recordings from complex mathematical structures. Many of the existing techniques are based on the assumption of independent and identically distributed functional observations. For time series of functional data, it is not only important to take into account the temporal dependence structure but also that this dependence structure is likely to change over time. The development of statistical methods that take into account the dependence structure has been a focal point in the literature on functional time series. To my knowledge, this thesis is however the first to address the necessity to develop theory and methodology for functional time series for which the assumption of weak stationarity is violated.

In Chapter 3, the concept of local stationarity was introduced for stochastic processes taking values in infinite-dimensional separable Hilbert spaces. The asymptotic framework that was developed in this chapter enables meaningful statistical inference and allows the derivation of large sample approximations of estimators and test statistics for functional processes of which the dynamics change over time. It 
moreover provides the building blocks for a well-defined spectral theory. A welldefined spectral theory is of foremost importance in many applications as it is often the frequency content of the process that is of interest. It moreover provides a complete characterization of the second-order dependence structure of the process. In the functional setting, this can especially be valuable because it is frequently unclear what specific model could describe the process. Chapter 3 therefore focused on the investigation of a class of processes for which a time-varying functional Cramér representation exists. The notion of a time-varying spectral density operator, which inherently corresponds to this representation, was investigated and a uniqueness property derived. It was furthermore shown that time-varying functional ARMA processes admit such a representation and that their second-order characteristics are completely characterized by the time-varying spectral density operator. Time-varying autoregressive processes were examined in detail and some time domain aspects, such as conditions for which a causal solution exists, were provided. Weakly stationary functional processes are comprised as a special case of the introduced framework and, as a consequence, the framework provides a meaningful generalization of spectral theory to nonstationary functional time series. The last part of the chapter focused on the estimation of the time-varying spectral density operator. It was shown that a consistent and asymptotically Gaussian estimator can be constructed by means of a smoothed version of the segmented periodogram operator. Crucial in this construction is the application of a data taper to the functional observations.

Chapter 3 can be seen to provide a theoretical skeleton for the development of statistical methods that take into account that the dependence structure of a functional time series possibly changes over time. Because the data is intrinsically infinite-dimensional, an important aspect in future work would be to investigate appropriate dimension reduction techniques. The proposed framework allows in particular to investigate how existing techniques such as functional dynamic principal component analysis should be adjusted when the process has time-varying spectral characteristics. Another important aspect would be to investigate what the violation of weak stationarity would mean for a finite-dimensional representation of the series. A localized Cramér Karhunen-Loève expansion might be considered.

In order to prevent model misspecification and drawing incorrect inferences it is important to test whether the assumption of temporal constancy of the secondorder structure, which lies at the basis of most statistical inference procedures, is in fact justified. This observation provided the motivation of Chapter 4 in which a method was proposed to test for stationarity of a functional time series. The test that was introduced exploits that the functional Discrete Fourier Transform evaluated at distinct Fourier frequencies are asymptotically uncorrelated if and only if the process is functional weakly stationary.

The method has some useful properties. No structural modeling assumptions are made other than weak structural modeling conditions in terms of functional cumulant tensors. This makes the method applicable to a wide range of functional time series, including those that are nonlinear and for which it is unclear what type of parametric model could represent the series. The method is also straightforward 
to apply in the finite-dimensional setting for which the specification of a linear model is not justifiable. The locally stationary framework that was introduced in Chapter 3 has moreover been used to investigate the sampling properties of the test under smoothly changing alternatives. The latter is a novelty as the existing literature on stationarity tests for functional time series are specifically designed to detect abrupt changes. Simulation results demonstrate the method is however also robust against this source of nonstationarity.

The final test statistic has a quadratic form and was derived to be chi-square distributed under both the null as well as under the alternative. Under the alternative, this distribution is a generalized chi-square with a non-centrality parameter. The expression for this mean function provides useful intuition behind the departure from stationarity. Knowledge on the sampling properties under the alternative of local stationarity seems especially beneficial in understanding how different degrees of nonstationarity affect the distribution. It will be interesting to compare how the method performs compared to existing time domain-based tests when the generating process is affected by a structural break. Additionally, application to real data still needs to be considered. Both will be pursued in the near future.

To summarize, the focal point in this thesis has been stochastic processes that are characterized by time-varying spectral characteristics. As highlighted in the introduction of this manuscript, the underlying motivation for all chapters is that it has become apparent that processes which exhibit nonstationary behavior are the rule rather than the exception in real life applications. This holds both for processes that are taking values in a Euclidean space as well as for processes that take values in a function space. The use of methods based on the assumption of temporal constancy can therefore risk forfeiting important information.

To analyze processes for which this assumption needs to be relaxed, this thesis focused on nonparametric methods in the frequency domain. The frequency domain allows for the complete characterization of the second-order dependence structure of a (functional) time series via the spectral density (operator). This characterization can be very valuable to capture the dependence structure when parametric models are inappropriate. Especially in the functional setting it is often unclear what type of model the process follows. Moreover, it has become apparent that a variety of parametric models show estimation problems when the dimension is too large. For example, determining the lag order $p$ of higher order functional autoregressive processes and estimation of the corresponding filter operators cannot seem to be done adequately. Not even under the assumption of stationarity. The temporal dependence can however be captured at once in the frequency domain without underlying structural modeling assumptions, making it a useful alternative to the time domain in this relatively new field of research.

The chapters that form the main body of this thesis considered both practical and theoretical aspects for the analysis of time-varying spectra of stochastic processes. The first part was concerned with the long-time open problem of bandwidth selection for the estimation of time-varying spectra. A problem often encountered by practitioners. The second part addressed the need for the development of theory and methodology for nonstationary stochastic processes on infinite-dimensional 
separable Hilbert spaces. Of course, the analysis in this thesis is far from complete but hopefully provides a meaningful contribution to the existing literature and will help the development of theory and empirical methods for nonstationary processes taking values both in finite- and infinite-dimensional separable Hilbert spaces. 



\section{Bibliography}

Antoniadis, A., Paparoditis, E. and Sapatinas, T. (2006). A functional waveletkernel approach for time series prediction. Journal of the Royal Statistical Society Ser. B 86, 837-857.

Antoniadis, A. and Sapatinas, T. (2003). Wavelet methods for continuous time predictions using hilbert-valued autoregressive processes. Journal of Multivariate Analysis 87, 133-158.

Aue, A., Dubart Norinho, D. and Hörmann, S. (2015). On the prediction of stationary functional time series. Journal of the American Statistical Association 110(509), 378-392.

Aue, A., Gabrys, R., Horváth, L. and Kokoszka, P. (2009). Estimation of a changepoint in the mean function of functional data. Journal of Multivariate Analysis 100, 2254-2269.

Aue, A., Horváth, L. and Kokoszka, P. (2013). Structural breaks in time series. Journal of Time Series Analysis 34, 1-16.

Aue, A. and van Delft, A. (2016). Testing for stationarity of functional time series in the frequency domain. Working paper, UC Davis, Maastricht University.

Bartlett, M. (1950). Periodogram analysis and continuous spectra. Biometrika $37,1-16$.

Becker, S. and Mathé, P. (2013). A different perspective on the propagationseparation approach. Electronic Journal of statistics 7, 2702-2736.

Benko, M., Härdle, W. and Kneip, A. (2009). Common functional component analysis. Annals of Statistics 37, 1-34.

Berkes, I., Gabrys, R., Horváth, L. and Kokoszka, P. (2009). Detecting changes in the mean of functional observations. Journal of the Royal Statistical Society Ser. B 71, 927-946.

Besse, P., Cardot, H. and Stephenson, D. (2000). Autoregressive forecasting of some functional climatic variations. Scandinavian Journal of Statistics 27, 673-687.

Besse, P. and Ramsay, J. (1986). Principal components analysis of samples functions. Psychometrika 51, 285-311. 
Bogachev, V. I. and Miftakhov, A. F. (2015). On weak convergence of finitedimensional and infinite-dimensional distributions of random processes. Preprint 15102, Collaborative Research Centre 701, University of Bielefeld.

Bosq, D. (2000). Linear Processes in Function Spaces. Springer, New York.

Bosq, D. (2002). Estimation of mean and covariance operator of autoregressive processes in banach spaces. Statistical inference for Stochastic Processes 5, 287306.

Bosq, D. and Blanke, D. (2007). Inference and Prediction in Large Dimensions. Wiley, Chichester.

Bowsher, C. G. and Meeks, R. (2008). The dynamics of economic functions: Modeling and forecasting the yield curve. Journal of the American Statistical Association 103, 1419-1437.

Brillinger, D. (1981). Time Series: Data Analysis and Theory. McGraw Hill, New York.

Brillinger, D. and Rosenblatt, M. (1967). Asymptotic theory of estimates of $k$-th order spectra. Proceedings of the Natioinal Academy of Sciences, USA 57, 206210 .

Brockwell, P. and Davis, R. (1991). Time Series: Theory and Methods. Springer, New York.

Cardot, H. and Sarda, P. (2006). Linear regression models for functional data. In S. Sperlich and G. Aydinli (eds), The Art of Semiparametrics, Springer, Heidelberg, pp. 49-66.

Cooley, J. and Tukey, J. (1965). An algortihm for the machine calculation of complex fourier series. Mathematics of Computation 19(90), 297-301.

Cramér, H. (1942). On harmonic analysis in certain functional spaces. Arkiv för Matematik, Astronomi och Fysik 28B, 1-7.

Cremers, H. and Kadelka, D. (1986). On weak convergence of integral functionals of stochastic processes with applications to processes in $l_{p}^{e}$. Stochastic Processes and Their Applications pp. 305-317.

Dahlhaus, R. (1983). Spectral analysis with tapered data. Journal of Time Series Analysis 4, 163-175.

Dahlhaus, R. (1988). Small sample effects in time series analysis:a new asymptotic theory and a new estimate. Annals of Statistics 16, 808-841.

Dahlhaus, R. (1993). Fitting time series models to nonstationary processes. Beiträge Zur Statistik No. 4, Universität Heidelberg . 
Dahlhaus, R. (1996a). On the kullback-leibler information divergence of locally stationary processes. Stochastic Process and their Applications 62(1), 139-168.

Dahlhaus, R. (1996b). Asymptotic statistical inference for nonstationary processes with evolutionary spectra. In: Robinson, P.M., Rosenblatt, M. (Eds.), Athens Conference on Applied Probability and Time Series Analysis, vol. II. SpringerVerlag, New York.

Dahlhaus, R. (1997). Fitting time series models to nonstationary processes. Annals of Statistics 25, 1-37.

Dahlhaus, R. (2009). Local inference for locally sationary time series based on the empirical spectral measure. Journal of Econometrics 151, 101-112.

Dahlhaus, R. and Polonik, W. (2009). Empirical spectral processes for locally stationary time series. Bernoulli 15, 1-39.

Dahlhaus, R. and Subba Rao, S. (2006). Statistical inference for time-varying arch processes. Annals of Statistics 34, 1077-1114.

Damon, J. and Guillas, S. (1982). The inclusion of exogeneous variables in functional autoregressive ozone forecasting. Environmetrics 13, 759-774.

Dauxois, J., Pousse, A. and Romain, Y. (1982). Asymptotic theory for the principal component analysis of a vector random function: some applications to statistical inference. Journal of Multivariate Analysis 12, 136-154.

Dehling, H. and Sharipov, O. (2005). Estimation of mean and covariance operator for banach space valued autoregressive processes with independent innovations. Statistical inference for stochastic processes 8, 137-149.

Dunford, N. and Schwartz, J. (1958). Linear Operators volume I. Wiley, New York.

Dwivedi, Y. and Subba Rao, S. (2011). A test for second-order stationarity of a time series based on the discrete fourier transform. Journal of Time Series Analysis 32(1), 68-91.

Edwards, R. (1967). Fourier series: a modern introduction. Holt, Rinehart and Winston, New York.

Eichler, M. (2007). A frequency-domain based test for independence between stationary time series. Metrika 65, 133-157.

Eichler, M., Motta, G. and von Sachs, R. (2011). Fitting dynamic factor models to non-stationary time series. Journal of Econometrics 163(1), 51-70.

Erbas, B., Hyndman, R. J. and Gertig, D. M. (2007). Forecasting age-specific breast cancer mortality using functional data models. Statistics in Medicine 26, 458470. 
Ferraty, F. and Vieu, P. (2006). Nonparametric Functional Data Analysis. Springer, New York.

Fiecas, M. and Ombao, H. (2016). Modeling the evolution of dynamic brain processes during an associative learning experiment. Journal of the American Statistical Association accepted manuscript.

Gabrys, R., Hörmann, S. and Kokoszka, P. (2010). Monitoring the intraday volatility pattern. Technical Report, Utah State University.

Grenander, U. (1981). Abstract Inference in: Wiley Series in Probability and Mathematical Statistics, Vol. IX. John Wiley \& Sons, New York.

Grenander, U. and Rosenblatt, M. (1957). Statistical analysis of time series. New York: Wiley.

Grinblat, L. S. (1976). A limit theorem for measurable random processes and its applications. Proceedings of the American Mathematical Society 61, 371-376.

Hallin, M. (1986). Nonstationary q-dependent processes and time-varying moving average models: invertibility properties and the forecasting problem. Advances in Applied Probability 32, 170-210.

Hays, S., Shen, H. and Huang, J. Z. (2012). Functional dynamic factor models with application to yield curve forecasting. Annals of Applied Statistics 6, 870-894.

Hörmann, S., Kidziński, L. and Hallin, M. (2015). Dynamic functional principal components. The Royal Statistical Society: Series B 77, 319-348.

Hörmann, S. and Kokoszka, P. (2010). Weakly dependent functional data. The Annals of Statistics 38(3), 1845-1884.

Horváth, L., Hušková, M. and Kokoszka, P. (2010). Testing the stability of the functional autoregressive process. Journal of Multivariate Analysis pp. 352-367.

Horváth, L. and Kokoszka, P. (2012). Inference for Functional Data with Applications. Springer-Verlag, New York.

Horváth, L.and Kokoszka, P. and Rice, G. (2014). Testing stationarity of functional time series. Journal of Econometrics pp. 66-82.

Hsing, T. and Eubank, R. (2015). Theoretical Foundations of Functional Data Analysis, with an Introduction to Linear Operators. Wiley, New York.

Hyndman, R. J. and Booth, H. (2008). Stochastic population forecasts using functional data models for mortality, fertility and migration. International Journal of Forecasting 24, 323-342.

Hyndman, R. J. and Ullah, M. S. (2007). Robust forecasting of mortality and fertility rates: A functional data approach. Computational Statistics and Data Analysis 51, 4942-4956. 
Karhunen, K. (1947). Über lineare methoden in der wahrscheinlichkeitsrechnung. Annales Academiae Scientiarium Fennicae, Ser. A.I. Math.-Phys. 37, 1-79.

Kleffe, J. (1973). Principal components of random variables with values in a separable hilbert space. Mathematische Operationsforschung Statistik 5, 391-406.

Künsch, H. (1995). A note on causal solution for locally stationary ar processes. Preprint, ETH Zurich.

Lee, J. and Subba Rao, S. (2016). A note on general quadratric forms of nonstationary stochastic processes. Technical Report, Texas A and M University.

Loève, M. (1948). Fonctions aléotoires du second ordre. Supplement to P. lévy, Processus stochastiques et Mouvement Brownian. Gauthier-Villars, Paris.

Martin, W. and Flandrin, P. (1985). Wigner-ville spectral analysis of nonstationary processes. IEEE Trans. Signal Process 33, 1461-1470.

Mas, A. (2000). Estimation d'opérateurs de corrélation de processus linéaires fonctionnels: lois limites, déviations modérées. Doctoral thesis, Université Paris VI.

Murphy, G. (1990). C*-algebras and Operator Theory. Academic Press.

Nelson, E. (1969). Topics in Dynamics, Volume I:Flows. University Press, Princeton.

Neumann, M. H. and von Sachs, R. (1997). Wavelet thresholding in anisotropic function classes and application to adaptive estimation of evolutionary spectra. The Annals of Statistics 25(1), 38-76.

Ombao, H., Raz, J., von Sachs, R. and Malow, B. (2001). Automatic statistical analysis of bivariate nonstationary time series. Journal of the American Statistical Association 96, 543-560.

Panaretos, V. and Tavakoli, S. (2013a). Cramer karhunen loeve representation and harmonic analysis of functional time series. Stochastic Processes and their Applications 123, 2779-2807.

Panaretos, V. and Tavakoli, S. (2013b). Fourier analysis of stationary time series in function space. The Annals of Statistics 41(2), 568-603.

Paparoditis, E. (2009). Testing temporal constancy of the spectral structure of a time series. Bernoulli 15, 1190-1221.

Polzehl, J. and Spokoiny, V. (2006). Propagation-separation approach for local likelihood estimation. Probability Theory and Related Fields 135(3), 335-362.

Preuß, P., Vetter, M. and Dette, H. (2011). Testing semiparametric hypotheses in locally stationary processes. Working paper, Ruhr-University Bochum.

Priestley, M. (1981). Spectral Analysis and Time Series, Vol. 2. Academic Press, London. 
Priestley, M. B. (1965). Evolutionary spectra and non-stationary processes. Journal of the Royal Statistical Society Ser. B 27(2), 204-237.

Priestley, M. B. and Rao, T. S. (1969). A test for non-stationarity of time-series. Journal of the Royal Statistical Society Ser. B 31, 140-149.

Ramsay, J. and Silverman, B. (2005). Functional Data Analysis. 2nd ed. edn, Springer, New York.

Schuster, A. (1897). On lunar and solar periodicities of earthquakes. Proceedings of the Royal Society of London 61, 455-465.

Sergides, M. and Paparoditis, E. (2009). Frequency domain tests of semiparametric hypotheses for locally stationary processes. Scandinavian Journal of Statistics 36, 800-821.

Subba Rao, T. (1970). The fitting of non-stationary time series models with timedependent parameters. Journal of the Royal Statistical Society. Series B (Methodological) 32, 312-322.

van Delft, A. and Eichler, M. (2015). Data-adaptive estimation of time-varying spectral densities.

URL: https://arxiv.org/pdf/1512.00825v2.pdf

van Delft, A. and Eichler, M. (2016). Locally stationary functional time series.

URL: https://arxiv.org/pdf/1602.05125v2.pdf

Vogt, M. (2012). Nonparametric regression for locally stationary time series. Annals of Statistics 40, 2601-2633.

von Sachs, R. and Neumann, M. (1999). A wavelet-based test for stationarity. Journal of Time Series Analysis 21, 597-613.

Yao, F., Müller, H.-G. and Wang, J, L. (2005). Functional linear regression for longitudinal data. Annals of Statistics 33, 2873-2903. 


\section{Valorization}

Since 2014, a valorization addendum is a mandatory part of all doctoral theses at Dutch Universities. Roughly speaking, the addendum should provide social justification of the research undertaken. More precisely, according to the National Valorization Committee the term valorization stands for "The process of creating value from knowledge by making knowledge suitable and/or accessible for economic and/or social exploitation and translating it into competitive products, services, processes and new activities". In this addendum, the knowledge valorization of the topic of this thesis - i.e., practical and theoretical aspects of time-varying spectral analysis on Hilbert spaces - is outlined. I shall do this by discussing how the topic and the respective findings of my doctoral thesis are of social and economic relevance.

This thesis has been centered around the concept of serially correlated stochastic processes - i.e., time series - in particular those with a changing correlation structure over time. With nonzero serial correlation we mean that a stochastic process - a process that is subject to random variation and evolves over time - is possibly influenced by its own past. Equivalently, a nonzero serial correlation structure implies that, if we decompose the process in terms of its cyclical behavior by representing it in the spectral domain, the energy that is dissipated by the process is not equally spread over frequencies. A socially and economically relevant example where the frequency content of a stochastic process is of importance can come from engineering. Decomposition of earthquake vibrations into its components allows to determine which vibrations, of different speeds and amplitudes, account for most variation in the signal. Buildings can then be designed to avoid interaction with the strongest components.

As stated in the introduction of this thesis, many physical phenomena, including the above example, exhibit nonstationary behavior as a result of smooth changes in their second-order structure. For example, in meteorology the atmospheric turbulence shows clear changes when measured over time. Atmospheric turbulence are the small, irregular air motions characterized by winds that vary in speed and direction. These turbulences affect how water vapor, energy and other substances such as smoke are distributed and therefore cause for instability in the atmosphere. Moreover, daily records of temperature, precipitation and cloud cover over a region as three related surfaces may change over time due to global climate changes. Anticipation to weather conditions is vital in different areas of society and can help reduce economic as well as social damages to a minimum. In order to optimally antici- 
pate to these conditions, weather prediction models need to take this nonstationary behavior into account.

In economics, empirical studies have shown that macroeconomic data, such as interest rates or variables related to the gross domestic product, exhibit smooth changes when measured over longer periods of time or on a fine enough time resolution. Central banks and other research institutions will have to integrate this behavior in their development of prediction models. This is of foremost importance since these models form the basis of an economic outlook and are consequently used by policy makers to adjust government spending and fiscal policy. Another important related example comes from finance, where implied volatility of an option as a function of moneyness changes over time. This information is relevant among others to investors, banks and insurance companies.

In medicine, different recording techniques of brain activity such as electroencepahlograms (EEG's), functional magnetic resonance images (fMRI) and local field potentials (LFP's) show that the underlying dynamics of the brain process have spectral properties that evolve over time. These changing dynamics are important to take into consideration in order to correctly model how, for example, different regions of the brain such as the nucleas accumbens or the hippocampus, are involved in certain cognitive processes. In chapter 2 , the introduced data-adaptive method was applied to local field potentials recordings.

These are only a few applications where the process is characterized by timevarying spectral characteristics. The list of disciplines in which such processes occur is however much longer and includes geophysics, astronomy, sound analysis and electrical engineering. Just as for the examples provided above, these disciplines are all involved in answering socially and economically relevant questions. To answer these questions, they rely on statistical tools and techniques that are made available by the statistical or econometric community or yet related fields. Failing to properly take into account the time-varying second-order characteristics will make the underlying model inappropriate and accordingly any inferences drawn from it invalid.

Despite of its importance, the majority of methods applied is still based on the assumption that the underlying data generating process is either time-invariant or that the nonstationarity is caused by abrupt changes. Another type of nonstationary processes that has received a considerable amount of attention in the (econometric) time series literature, are so-called integrated processes. These can be classified by the property that the differenced series are stationarity. These types of nonstationary processes have in common that their analysis generally does not require a completely different framework to derive statistical properties. This in contrast to the case where the second-order structure is varying over time.

As made clear by the wide range of disciplines listed above, changes in the second-order structure are however the rule rather than the exception. It was already mentioned in the introduction of this thesis that the extension of many estimation methods to processes of which the data generating mechanism is in a constant state of change, is not a natural one. Not only will the classical theoretical framework - on which many statistical inference procedures are based - became meaningless, the decomposition in terms of frequency components might no longer 
have the same physical interpretation. In many applications, the research question however requires knowledge on how the process dissipates energy over different frequency components. A clear example of this was provided at the beginning of this addendum, where we motivated the relevance to correctly decompose earthquake vibrations. Even when the research question is not directly formulated in terms of its frequency content, it can often still be advantageous to proceed the analysis of the series in the spectral domain. The second-order dependence structure of a time series can be completely characterized in the spectral domain via the spectral density (operator). Especially when one is facing high-dimensional data, when parametric modeling cannot be justified or when the process exhibits cyclical behavior, the spectral domain can provide a useful alternative to the time domain.

Relatively recent (Dahlhaus, 1996a) a framework for finite-dimensional time series with time-varying characteristics was introduced that allows for both meaningful statistical inference as well as for a spectral theory in which concepts such as 'frequency' and 'energy' keep the same physical interpretation. Although this has led to a surge in the literature on processes with time-varying spectral characteristics, there are still many open problems. An important one is the problem to estimate the time-varying spectrum in practice. Because this object is a function of both time and frequency and because theoretical results are not directly applicable, the practitioner is forced to face the uncertainty principle. That is, the practitioner needs to find the right balance between the required estimation precision in time and frequency direction, given that more precision in one direction directly means a loss of precision in the other direction.

Another more recent problem is that the surge in data storage techniques has led to the need for models that can deal with data that are intrinsically infinitedimensional. In effect, many modern datasets can be viewed as sampled recordings from complex mathematical structures such as curves or surfaces. The extension of methods that are suitable in finite-dimensional spaces to methods that are suitable in infinite-dimensional spaces is far from straightforward and requires careful consideration of various convergence concepts as well as the consideration of appropriate dimension reduction techniques. The field of research involved with this during the past few decades is known as functional data analysis. A particular subfield, known as functional time series, is concerned with ordered collections of functional data and focuses on the development of methods that take into account the second-order dependence structure. These methods rely however on the assumption that this dependence structure remains constant over time and thus that the series is functional weakly stationary. Not unlike the finite-dimensional setting, this assumption turns out to be too restrictive in many applications. The aforementioned meteorological elements as related surfaces as well as the implied volatility surface are examples of functional time series with changing second-order characteristics. The development of statistical techniques to model this type of functional data correctly has however remained an open problem.

This thesis has addressed these two open problems. That is, this thesis has been concerned with both the practical problem of the estimation of time-varying spectral densities as well as the need for the development of theory and methodology for 
infinite-dimensional stochastic processes that are characterized by changing secondorder characteristics. In Chapter 2, a data-adaptive approach was introduced for the estimation of time-varying spectra. Without going into details again, the method allows the data to tell the practitioner what would be the 'correct' balance between time- and frequency resolution for each time-varying spectral variate in the timefrequency plane. The algorithm as developed will be provided as a software package and will therefore be easily accessible to any practitioner that is facing this problem. Chapter 3 was devoted to develop spectral theory and methodology to model functional time series that have time-varying spectral characteristics. Although the results of Chapter 3 are theoretical and not of a nature that the practitioner can apply directly, they are a necessary step in understanding the behavior of these type of processes. This in turn is a necessary step in the development of methods that will become available to the practitioner. An important aspect of research is that it paves the way for future research. This chapter does exactly that by providing the foundation for a framework that enables statistical inference of nonstationary functional time series and that allows the derivation of large sample approximations of estimators and test statistics. Chapter 4 builds in fact on the framework of Chapter 3 by providing a test statistic that allows to test for the presence of time-varying spectral characteristics. This test allows the practitioner to determine whether standard inference methods can be used or that these become invalid and that alternative methods based on the framework as proposed in Chapter 3 need to be considered in order to draw correct inferences.

I hope the knowledge valorization provided in this addendum will make clear the importance of the research undertaken in this dissertation. An outlook into possible future research has been provided in both the conclusion of the relevant chapters as well as in the overall conclusion (Chapter 5). These projects, as well as the implementation of the proposed methods into easily accessible software packages, are something I look forward to work on in the near future. 


\section{Nederlandse samenvatting}

Veel fysische verschijnselen vertonen niet-stationair gedrag ten gevolge van geleidelijke veranderingen over tijd in de tweede-orde afhankelijkheidsstructuur. Voorbeelden zijn te vinden in een groot aantal verschillende wetenschappelijke disciplines zoals in de geofysica, geneeskunde, economie en ingenieurswetenschappen. Bovendien kunnen veel moderne datasets, die mogelijk onderhevig zijn aan dit type gedrag, gezien worden als processen die variëren over een continuüm. In dit proefschrift staan stochastische processen centraal die gekarakteriseerd kunnen worden door beide aspecten. Met andere woorden, dit proefschrift is gewijd aan het ontwikkelen van theorie en methodologie voor de analyse van stochastische processen waarvan de afhankelijkheidsstructuur van de tweede-orde, en dus de spectrale karakteristieken, veranderen over tijd. De elementen van het stochastische proces worden verondersteld waarden aan te nemen op een separabele Hilbert-ruimte en zijn dus van een intrinsiek oneindige dimensie.

Een stochastisch proces - een evolutie proces dat bestaat uit een reeks geordende toevalsvariabelen - wordt zwak stationair genoemd of stationair van de tweede-orde wanneer de eerste twee momenten van de afhankelijkheidsstructuur, d.w.z. de verwachting en covariantie, niet tijdsafhankelijk zijn. Een zwak stationair stochastisch proces kan alternatief worden weergegeven als een stochastisch signaal met willekeurige amplitudes and fases die zelf tijdsonafhankelijk zijn. Deze decompositie in termen van harmonische trillingen met tijd constante coëfficiënten wordt ook wel de spectrale representatie van het proces genoemd. Het bijbehorende spectrum of spectrale dichtheidsfunctie van het proces - de Fourier transformatie van de covariantie structuur - geeft informatie over hoe de energie in het proces verdeeld is, of wordt afgegeven, over verschillende frequenties.

Ondanks het feit dat het duidelijk is dat de tweede-orde afhankelijkheidsstructuur in vele gevallen wel degelijk verandert over tijd, is het merendeel van de statistische methoden die alledaags worden toegepast gebaseerd op de aanname van een onderliggend tijdsinvariant generatiemechanisme. Wanneer de tweede-orde afhankelijkheidsstructuur tijdsafhankelijk is, houdt het postulaat van zwakke stationariteit niet. Dit heeft twee belangrijke gevolgen. Ten eerste is een spectrale representatie van het proces waarbij de fysische interpretatie van 'frequentie' en 'energie' behouden blijft, niet per se definieerbaar. Ten tweede is een alternatieve asymptotische theorie noodzakelijk voor betekenisvolle statistische inferentie.

Voor niet-stationaire processen waarvan de elementen waarden aannemen in Euclidische ruimtes, is er een raamwerk (Dahlhaus, 1996a) geïntroduceerd die zowel een betekenisvolle statististische inferentie mogelijk maakt alsmede een spectraal theo- 
rie waarin concepten als 'frequentie' and 'energie' behouden blijven. Dit raamwerk heeft de weg vrij gemaakt voor toenemend onderzoek naar processen waarvan de afhankelijkheidsstructuur onderhevig is aan veranderingen over tijd. Veel problemen zijn echter tot op heden onopgelost gebleven. Een belangrijk probleem in de praktijk is het schatten van het tijdsafhankelijk spectrum. Omdat deze functie niet alleen afhangt van frequentie maar ook van tijd en theoretische resultaten niet direct toepasbaar zijn, is men in de praktijk geconfronteerd met het onzekerheidsprincipe. Een balans moet daarbij gevonden worden tussen de benodigde precisie in beide richtingen, waarbij meer schattingsprecisie in de ene richting ten koste gaat van de schattingsprecisie in de andere richting.

Een ander zeer urgent probleem doet zich voor als gevolg van recente technologische ontwikkelingen. Ook al variëren veel fysische verschijnselen over een continuüm zoals over een kromme of een oppervlakte, pas zeer recente ontwikkelingen hebben het mogelijk gemaakt om deze processen waar te nemen op willekeurig hoge frequenties. De hierdoor toenemende vraag naar methodologie die de onderzoeker in staat stelt om informatie te extraheren en conclusies te trekken uit data die gezien kunnen worden als steekproefsgewijze metingen van complex wiskundige structuren, is het brandpunt van functionale data analyse. Het merendeel van de bestaande functionale data literatuur neemt aan dat de functionale observaties onafhankelijk en identiek verdeeld zijn. Net als voor stochastische processen waarvan de toevalsvariabelen waarden aannemen in eindig dimensionale vectorruimten, is het voor tijdreeksen van functionale data van groot belang om statistische methodes te ontwikkelen die niet alleen rekening houden met de inherente afhankelijkheidsstructuur, maar ook met het feit dat deze structuur zeer waarschijnlijk verandert over tijd. Tot nu toe zijn er echter enkel statistische methoden en instrumenten ontwikkeld onder het postulaat van zwakke stationariteit.

Bovengenoemde problemen vormen de kern van het gedane onderzoek in dit proefschrift. In het eerste gedeelte (Hoofdstuk 2) van dit proefschrift wordt een dataadaptieve methode ontwikkeld voor de schatting van spectra van niet-stationaire processen. Verondersteld wordt dat het niet-stationaire gedrag niet alleen veroorzaakt kan worden door geleidelijke veranderingen maar tevens door structurele veranderingen. De geïntroduceerde schattingstechniek is gemotiveerd vanuit het feit dat optimale bandbreedtes van de vensterfuncties in tijd- en frequentie richting, respectievelijk, afhangen van de gladheidseigenschappen van het ware onbekende spectrum in deze richtingen. In de praktijk zijn de optimale waarden van deze bandbreedtes niet direct toepasbaar. Tegelijkertijd is de schattingsprecisie sterk afhankelijk van de lokalisatie in tijd- en frequentie richting. Een data-adaptieve techniek om deze brandbeedtes optimaal te selecteren is tot nu toe een onopgelost probleem gebleven. Het streven is om hier een oplossing voor te vinden door een methode te introduceren die het mogelijk maakt om de optimale vorm van de vensterfunctie - beschouwd als een tweedimensionale functie in het tijd-frequentie vlak - op een data-adaptieve manier vast te stellen. Het onderliggende principe van het algoritme is om iteratief de bandbreedtes van de vensterfunctie uit te breiden totdat 'homogeniteit' wordt geschonden. Dit vindt plaats in de separatie fase die bewerkstelligd wordt door middel van een extra vensterfunctie, een sanctievenster. Dit sanctievenster 
geeft minder of geen gewicht aan de coördinaten binnen de bandbreedtes die niet tot dezelfde homogene regio behoren als het coördinaat waarvoor de vensterfunctie wordt vastgesteld. Om volledige flexibiliteit te kunnen realiseren voor de graad waarmee de fluctuaties worden afgezwakt in de respectievelijke richtingen, worden de schattingen van de locale spectrale coördinaten verwezenlijkt door de adaptieve venster functies toe te passen op de pre-periodogram functie. Dit maakt het mogelijk om tegelijkertijd relatief hoge resolutie in tijd- en frequentie richting te kunnen verkrijgen. Dit is moeilijker te bewerkstelligen voor schatters die gebaseerd zijn op gesegmenteerde periodogram functies. De flexibiliteit die het pre-periodogram biedt gaat daarentegen gepaard met nadelige artefacten zoals het mogelijk aannemen van de pre-periodogram functie van negatieve waarden. Een specifieke functionaliteit van het algoritme, geïntegreerd in een stabiliteitsfase, is dat het dit nadelige effect kan beperken. Een simulatie studie is uitgevoerd om de prestatie van het algoritme te onderzoeken. De methode is vervolgens getoetst op neurowetenschappelijke data.

De noodzaak om voor functionale tijdreeksen de veronderstelling van zwakke stationariteit te kunnen afzwakken ligt ten grondslag aan het onderzoek in Hoofdstuk 3. Een theoretisch raamwerk wordt geïntroduceerd dat het mogelijk maakt om statistische inferenties te trekken van tijdreeksen van functionale data waarvan de onderliggende dynamieken veranderen over tijd. Het concept lokale stationariteit wordt gedefinieerd voor functionale tijdreeksen en een spectraal theorie wordt ontwikkeld. Meer specifiek, een tijdsafhankelijke functionale spectrale representatie wordt afgeleid en een klas van functionale processen wordt vastgesteld die gedefinieerd kunnen worden door middel van deze representatie. Vervolgens wordt bewezen dat deze klas voldoet aan de geïntroduceerde definitie van funtionale lokale stationariteit. De notie van een tijdsafhankelijke spectrale dichtheidsoperator is geïntroduceerd en de eigenschappen onderzocht. Naar analogie met de multivariate tijdsreeksen kan dit object uniek gedefinieerd worden. Tijdsafhankelijke functionale ARMA processen worden bestudeerd in detail. Aangetoond wordt dat deze type processen behoren tot de geïntroduceerde klas en dus kunnen worden weergegeven via een tijdsafhankelijke functionale spectrale representatie. In deze analyse wordt onderscheid gemaakt tussen transfer operatoren - generalisaties van de Fourier transformaties van de reeks moving average matrices - die begrensd zijn in standaard operator norm en die begrensd zijn in Hilbert-Schmidt norm. Het raamwerk wordt in het laatste gedeelte van dit hoofdstuk toegepast om een schatter van de tijdsafhankelijke spectrale dichtheidsoperator te construeren. Deze schatter is gebaseerd om een functionale versie van een gesegmenteerde periodogram matrix. Eigenschappen als consistentheid en de asymptotische verdeling van deze schatter worden ten slotte in detail bestudeerd.

Wanneer de aanname van zwakke stationariteit niet geldig is, is de toepassing van de meeste statistische inferentie methodes die bestaan voor functionale tijdreeksen niet langer gepast. Dit kan namelijk resulteren in zware misspecificatie van modellen en daardoor tot incorrecte inferentie. Het is daarom uitermate belangrijk om een gepaste toets te ontwikkelen die het mogelijk maakt om na te gaan of de hypothese van temporele constantheid voor functionale tijdreeksen legitiem is. Hoofdstuk 4 is gewijd aan dit onderwerp en introduceert een spectraal domein-gebaseerde toets waarmee deze hypothese onderzocht kan worden. De toets gebruikt dat de functio- 
nale Discrete Fouriertransformatie, wanneer geëvalueerd in afzonderlijke frequenties, een asymptotisch ongecorreleerde reeks vormt als en alleen als het proces functionaal zwak stationair is. De toetsingsgrootheid is gebaseerd op een empirische covariantie operator van deze functionale Discrete Fouriertransformaties, die vervolgens geprojecteerd wordt. De methodologie is theoretisch gerechtvaardigd door middel van asymptotische resultaten. De asymptotische eigenschappen van de toetsingsgrootheid worden afgeleid onder de nul- en de alternatieve hypothese van lokaal stationair functionale tijdreeksen. Hoofdstuk 4 bouwt daarmee voort op het theoretisch raamwerk dat geïntroduceerd werd in Hoofdstuk 3. Er worden geen structurele model aannames gemaakt behalve dan zwakke mixing voorwaarden in de vorm van functionale versies van de klassieke cumulant mixing voorwaarden. Een simulatie studie is uitgevoerd om de prestatie van de toetsingsgrootheid te onderzoeken in steekproeven van eindige populaties. 


\title{
Curriculum Vitae
}

\begin{abstract}
Anne van Delft was born on December 4, 1988 in Zoetermeer, The Netherlands. She studied Econometrics and Operations Research at Maastricht University. In August 2012, she obtained her M.Sc. with distinction (cum laude). After graduation, Anne joined the Department of Quantitative Economics as a Ph.D. candidate in September 2012, under the supervision of Dr. Michael Eichler and Prof. dr. Jean-Pierre Urbain. During the spring semester of 2016, Anne spent a couple of months at the University of California, Davis as a visiting Ph.D. student under the supervision of dr. Alexander Aue. The results of her research are presented in this thesis. Anne presented her work at various international conferences and universities and parts of this thesis have been submitted to international refereed academic journals.
\end{abstract}




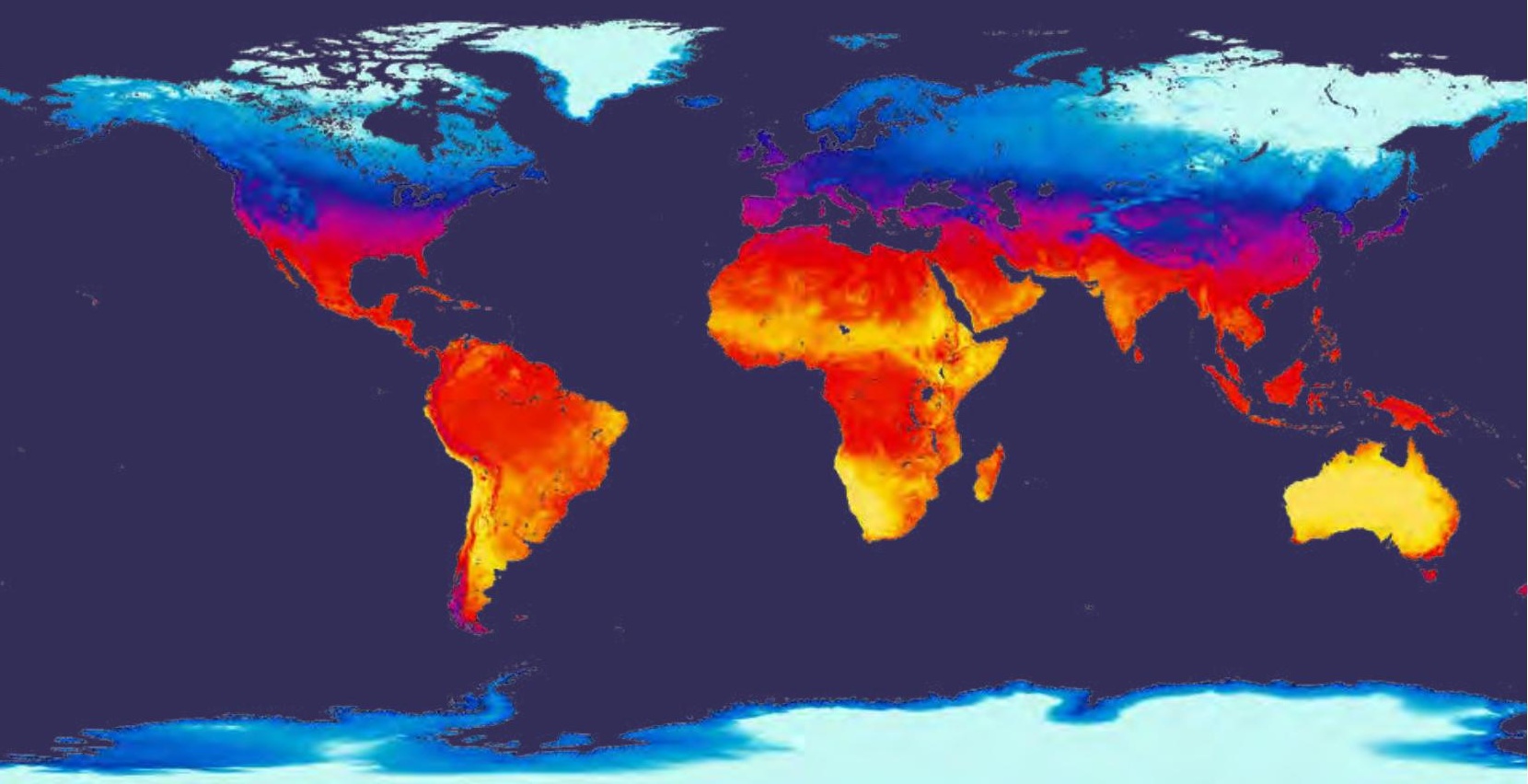

